\title{
DEVELOPMENT OF PERFORMANCE FUNCTIONS FOR ECONOMIC PERFORMANCE ASSESSMENT OF PROCESS CONTROL SYSTEMS
}

by

\section{Donghui Wei}

\author{
Submitted in partial fulfilment of the requirements for the degree \\ Philosophiae Doctor (Electronic Engineering) \\ in the
}

Faculty of Engineering, Built Environment and Information Technology

UNIVERSITY OF PRETORIA

February 2010 


\title{
UNIVERIIEIT VAN PRETORIA \\ UNIVERSITY OF PRETORIA \\ Development UUNIBESITHI YA PRETORIA UT Pertormance Functions \\ For Economic Performance Assessment \\ Of Process Control Systems
}

by

\section{Donghui Wei}

\author{
Promotor: Prof. Ian K. Craig \\ Department: Electrical, Electronic and Computer Engineering \\ Faculty of Engineering, Built Environment and Information Technology \\ Degree: PhD (Electronic Engineering)
}

\section{Summary}

Economic performance assessment (EPA) of control systems is receiving increasing attention in both academia and industry. It addresses the estimation of the potential benefits resulting from control upgrade projects and monitoring and improvement of economic performance of the control system.

Economic performance of control systems can often be related to crucial controlled variables dynamically and when controlled variables move away from an optimal operating point either more profit will be made or more cost will be incurred. This relation can be modelled by performance functions $(\mathrm{PFs})$. When the multivariate nature of a process's economic model is not considered, PFs of different controlled variables are referred to as individual performance functions. Otherwise, PFs of dependent controlled variables are referred to as joint performance functions.

PFs play an important role in the latest techniques of EPA. There appears, however, to be no systematic method for developing PFs. The lack of such a method restrains further research into EPA, as without well-established PFs an EPA cannot be conducted smoothly and therefore cannot effectively support decision-making for management. The development of PFs is a bottleneck in the further research into EPA. Furthermore, the multivariate nature of processes has not been taken into account sufficiently as far as the relevant literature is concerned, which hampers the accuracy of PFs and accordingly the accuracy of economic assessment results.

The contributions of this thesis lie in the following aspects:

- A methodology for developing PFs is proposed, based on the PF development for an electric arc furnace, a grinding mill circuit and a stage of a bleach plant.

- A comprehensive case study of an EPA of three controllers of a grinding mill circuit is conducted using a newly published framework to show the significance of PFs and how to perform an EPA systematically.

- The current practice and guidelines on the control and functional/economic performance 
assessment of grinding mill circuits are captured using a survey study.

The multivariate nature of an electric arc furnace's economic model is investigated and joint performance functions are built based on individual performance functions. A multivariate economic assessment is conducted that shows how joint performance functions can help to provide a more accurate estimate of the economic performance of a controlled process.

A web-based survey study on grinding mill circuits in mineral processing industries is conducted. One of its objectives is to obtain general PFs of grinding circuits. The survey results provide instructive insight into the PFs of grinding circuits. Furthermore, an in-depth literature review is conducted and the relationship between the product's particle size distribution of grinding mill circuits and mineral recovery in downstream flotation circuits is revealed. The PFs of a grinding mill circuit being considered are formed, based on the survey results and literature study. An investigation into the PF development of a stage of a bleach plant is performed and crucial ideas used for their development are abstracted.

A methodology for developing PFs for the EPA of control systems is then proposed by synthesising the methods used in the PF development described above. This methodology mainly includes the following stages:

Stage 1: Determine information required for PF development.

- Process operation and control understanding.

- Process economics understanding.

Stage 2: Gain required information on PF development.

- PF-related information elicitation using survey research.

- PF-related information available in the literature, including textbooks, journal papers, conference papers.

- PF-related information from plant tests.

Stage 3: Obtain suitable performance measures.

Stage 4: Make suitable assumptions.

Stage 5: Determine PFs.

Stage 6: Develop Joint PFs.

An economic assessment of three controllers (a nonlinear model predictive controller, a decentralised controller and three single-loop proportional-integral-derivative controllers) of the considered grinding mill circuit is conducted, using an EPA framework published recently to show the central role of PFs in the EPA and how to perform an EPA systematically. The circuit's PFs, developed as described above, are used for the assessment. The EPA also shows that the improvement in the economic performance with the nonlinear model predictive controller mainly results from the improvement of the operating point and the controlled variables' variation reduction only contributes a small part to the overall improvement, due to the characteristic of the PF of the circuit's product particle size distribution.

In addition, a web-based survey study is conducted and the current practice and guidelines on the control and functional/economic performance assessment of grinding mill circuits are captured. The questionnaire used for the study includes five segments. The first part identifies the respondents and the second part is intended to obtain background information on the milling circuits. The third part concerns the choice of key process variables and their economic impact. Part four involves the control of milling circuits and control loop performance and part five covers economic issues. 
Keywords: Economic performance assessment, performance functions, (joint/individual) performance functions, grinding mill circuits, electric arc furnace, bleach, process control, process economics. 


\section{Acknowledgement}

Studying abroad in a non-native language is a big challenge. The chance to master research skills and at the same time to be exposed to a completely different culture is fantastic. This chance was enhanced by doing research at the University of Pretoria, South Africa. The people and the atmosphere here made my study enjoyable.

It is there I met Professor Ian K. Craig, who has had and will continue to have a considerable influence on my life. He is not just a professional advisor, but also a good friend. His ideas have remained a constant source of inspiration, and our endless discussions have nurtured and inspired me. I would like to express my most sincere gratitude to him for his ever-present support, encouragement and guidance. Thanks for widening my views and helping me achieve my goals.

Thanks also to Prof. X. Xia, who brought me here, to a place I will never forget in my life. He has also given me significant instruction and support.

Thanks to all the friends I met in Pretoria: Miss Y. Li, Mr. M. Chen, Mr. X. Liang, Dr. J. Zhang and many others. I also want to extend my appreciation to Loutjie Coetzee for his great help in various aspects. They have helped me through difficult moments and I learned a great deal from them.

I am in debt to my family in P. R. China. It has been hard to stay far from them, but the distance strengthens our connection even more. I would like to thank my beloved wife, $\mathrm{Ou} \mathrm{Li}$, for her persistent love, patience and encouragement. To them I owe everything I gained. 


\section{Contents}

1 Introduction $\quad 1$

1.1 Motivation of economic performance assessment . . . . . . . . . . . . 1

1.2 A brief history of economic performance assessment . . . . . . . . . . . . 2

1.3 Overview of the current literature . . . . . . . . . . . . . . . 2

1.3.1 Origins of benefits from control systems and problems involved in economic performance assessment . . . . . . . . . . . . 3

1.3.2 'Warren family' . . . . . . . . . . . . . . . . . . 3

1.3.3 'Integral family' . . . . . . . . . . . . . . . . . . . . . . 4

1.3.4 Other methods . . . . . . . . . . . . . . . . 5 5

1.3.5 A framework of economic performance assessment . . . . . . . . . 6

1.4 Research objectives and approaches . . . . . . . . . . . . . . . . . 6

1.4.1 Research objectives . . . . . . . . . . . . . . 6

1.4 .2 Research approaches . . . . . . . . . . . . . . . . 6

1.5 Contributions . . . . . . . . . . . . . . . . . . 7

1.6 Organisation of thesis . . . . . . . . . . . . . . . . . 8

$\begin{array}{llr}2 & \text { Preliminary economic analysis } & 9\end{array}$

2.1 Process operation and control understanding . . . . . . . . . . . . 9

2.2 Identification of possible benefit . . . . . . . . . . . . . . 10

2.2 .1 Higher throughput . . . . . . . . . . . . . . . . . . 10 
2.2.3 Better yield . . . . . . . . . . . . . . . . . . . 11

2.2.4 Fewer unwanted byproducts . . . . . . . . . . . . . . . . 11

2.2 .5 Less labour . . . . . . . . . . . . . . . . . . . . 12

2.2 .6 Better quality . . . . . . . . . . . . . . . . . . 12

2.2.7 Quantifiable benefits from reducing pollution . . . . . . . . . . . . . 12

2.2.8 Quantifiable benefits from improving safety . . . . . . . . . . . . . 13

2.3 Conclusion . . . . . . . . . . . . . . . . . . . . . . . . 13

3 Review of a framework on economic performance assessment 14

3.1 Introduction . . . . . . . . . . . . . . . . . . . . . . 14

3.2 Step 1: Base case identification . . . . . . . . . . . . . . . . . . 14

3.2.1 Collect base case data of controlled variables of interest . . . . . . . . . . 14

3.2.2 Estimate the base case's probability density functions . . . . . . . . . 15

3.2.3 Develop performance functions $(v(y)) \ldots \ldots \ldots \ldots$

3.2.4 Calculate the economic performance index of the base case $\left(\Psi_{\text {base }}\right) \ldots \ldots 17$

3.2.5 Estimate the minimum variance $\left(\sigma_{m v}^{2}\right) \ldots \ldots \ldots \ldots$

3.2.6 Determine the operating point $\left(\mu_{m v}\right)$ of $\mathrm{CVs}$ for $\sigma_{m v}^{2} \ldots \ldots \ldots$

3.2.7 Calculate the maximum achievable performance $\left(\Psi_{m v}\right) \ldots \ldots$. . . . . 22

3.3 Step 2: Advanced controller design . . . . . . . . . . . . . . . . . 22

3.4 Step 3: Estimation of performance and initial economic assessment . . . . . . . 23

3.4.1 Estimate the standard deviation improvement $\left(\sigma_{\text {esti }}-\sigma_{\text {base }}\right)$ due to the advanced controller . . . . . . . . . . . . . . 23

3.4.2 Determine the optimal operating point $\left(\mu_{\text {esti }}\right)$ for the advanced controller 23

3.4.3 Estimate the economic performance index with the advanced controller

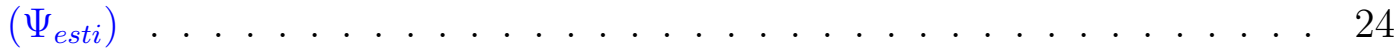

3.4.4 Do fixed cost estimation . . . . . . . . . . . . . . . . . 24 
3.4.5 Conduct initial economic project evaluation and decision making . . . . . 24

3.5 Step 4: Implementation of the advanced controller . . . . . . . . . . . . . 26

3.6 Step 5: Calculation of performance and success assessment . . . . . . . . . 26

3.6.1 Collect data of controlled variables of interest . . . . . . . . . . 26

3.6.2 Determine the probability density functions of controlled variables . . . . 27

3.6.3 Calculate the economic performance index of the advanced controller $\left(\Psi_{\text {adv }}\right) 27$

3.6.4 Calculate the achieved profit $\left(\Psi_{\text {adv }}-\Psi_{\text {base }}\right) \ldots \ldots \ldots$. . . . . . 27

3.6.5 Derive the new optimal operating point $\left(\mu_{a d v 2}\right)$ based on the standard deviation obtained in Step $2.3\left(\sigma_{a d v}\right) \ldots \ldots . . . . . . . . . . .27$

3.6.6 Update the minimum variance $\left(\sigma_{m v 2}^{2}\right)$ estimate and maximum achievable performance $\left(\Psi_{m v 2}\right) \ldots \ldots \ldots \ldots$. . . . . . . . . . . . . 27

3.6.7 Do statistical experiments to examine economic improvement . . . . . . . 28

3.6.8 Conduct final economic project evaluation . . . . . . . . . . . . . . . . . 29

3.7 Conclusion . . . . . . . . . . . . . . . . . . . . . . 29

4 Performance functions and a multivariate economic assessment of a furnace 30

4.1 Introduction . . . . . . . . . . . . . . . . . . . 30

4.2 Process background and economic model . . . . . . . . . . . . . . . 31

4.2.1 Description of the electric arc furnace . . . . . . . . . . . . . 31

4.2.2 Economic performance index of the electric arc furnace . . . . . . . . . 32

4.2 .3 Process variables and control strategies . . . . . . . . . . . . . 32

4.3 Review of the development of individual performance functions of the furnace . 32

4.3 .1 Percentage carbon $\ldots \ldots \ldots \ldots . \ldots \ldots$

4.3.2 Steel temperature . . . . . . . . . . . . . . . . . . 34

4.3 .3 Off-gas temperature . . . . . . . . . . . . . . . . . 34

4.3 .4 Slag foam depth . . . . . . . . . . . . . . . . 35

4.3 .5 Steel mass . . . . . . . . . . . . . . . . . . . . . 35 
4.3 .7 Relative furnace pressure . . . . . . . . . . . . . . . . . . . 36

4.4 Development of joint performance functions of the furnace $\ldots \ldots$. . . . . 36

4.4.1 Determining the dependence of controlled variables . . . . . . . . . . 37

4.4.2 Developing the joint performance functions for dependent controlled vari-

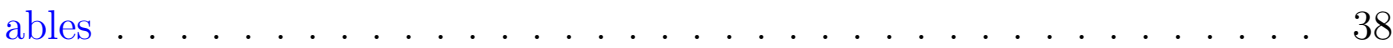

4.5 Methodology for the economic assessment of the furnace controllers . . . . . . 40

4.6 Economic assessment of a model predictive controlled furnace . . . . . . . . . 42

4.6.1 Overall average economic performance . . . . . . . . . . . . . . 42

4.6.2 Hypothesis test . . . . . . . . . . . . . . . . . . . . 43

4.7 Conclusion . . . . . . . . . . . . . . . . . . . . . . 44

5 State-of-art of control and performance assessment of grinding mill circuits 45

5.1 Introduction . . . . . . . . . . . . . . . . . . . 45

5.2 Process description of grinding mill circuits . . . . . . . . . . . . . . 45

5.3 Primary survey theory and techniques . . . . . . . . . . . . . . 47

5.3 .1 An overview of survey research . . . . . . . . . . . . . . 47

5.3 .2 Developing ideas for survey research . . . . . . . . . . . . . . . 48

5.3.3 Advantages, importance and objectives of questionnaires . . . . . . . . . 49

5.3 .4 Designing questionnaires . . . . . . . . . . . . . . . 50 50

5.4 Research methodology and questionnaire design . . . . . . . . . . . . 56

5.4.1 Objectives of and population for the survey research . . . . . . . . 56

5.4 .2 Sample selected in the survey research . . . . . . . . . . . 56

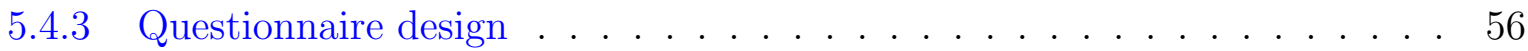

5.4 .4 Conducting of the survey research . . . . . . . . . . . . 57

5.5 Survey results: Background information on grinding circuits . . . . . . . . 58 
5.5.2 Method for measuring variables . . . . . . . . . . . . . . . 60

5.5.3 Actuators used for manipulating process variables . . . . . . . . . 63

5.5.4 Other background information . . . . . . . . . . . . . . . 64

5.6 Survey results: Choice of crucial process variables and their economic impact . . 65

5.6.1 Choice of key process variables . . . . . . . . . . . . . 65

5.6.2 Setpoint choice, corrective action and the economic impact of the product particle size . . . . . . . . . . . . . . . 66

5.6.3 Setpoint choice, corrective action and the economic impact of the sump level ........................ 6 67

5.6.4 Setpoint choice, corrective action and the economic impact of the mill load 68

5.6.5 Setpoint choice, corrective action and the economic impact of the sump discharge slurry density . . . . . . . . . . . . . . . . . 69

5.6.6 Performance functions of the controlled variables . . . . . . . . . . . 70

5.6 .7 Dependence of controlled variables . . . . . . . . . . . . . 71

5.7 Survey results: Functional performance assessment of grinding circuits . . . . . . 71

5.7.1 Frequency of monitoring the control loop performance . . . . . . . . . 72

5.7 .2 Satisfaction with control loop performance . . . . . . . . . . . 72

5.7.3 Main problems confronted in the control or optimisation of the grinding mill circuit . . . . . . . . . . . . . . . . . 72

5.8 Survey results: Economic performance assessment of grinding mill circuits . . 72

5.8.1 Methods for estimating benefit . . . . . . . . . . . . 73

5.8 .2 Cost estimation methods . . . . . . . . . . . . . . 73

5.8.3 Importance and accuracy of, and satisfaction with economic assessment . 73

5.8.4 Most important topics requiring additional development . . . . . . . . . 74

5.9 Conclusion . . . . . . . . . . . . . . . . . . . . . 74 
6.1 Introduction . . . . . . . . . . . . . . . . 76

6.2 Obtaining performance function-related information from a plant test . . . . . 77

6.3 Assumptions used for the performance function development . . . . . . . . . 77

6.4 Performance functions of a given grinding circuit . . . . . . . . . . . . . 78

6.4.1 Process description and process variables . . . . . . . . . . 78

6.4.2 Performance function for the particle size distribution . . . . . . . . . 79

6.4.3 Performance function for the mill load . . . . . . . . . . . . . . 81

6.4.4 Performance function for the sump level . . . . . . . . . . . . . 82

6.5 Overall economic performance of the circuit . . . . . . . . . . . . 83

6.6 Conclusion . . . . . . . . . . . . . . . . . . . . . . 84

7 Economic performance assessment of three milling circuit controllers $\quad 85$

7.1 Introduction . . . . . . . . . . . . . . . . . . . 85

7.2 Models and control objectives of the milling circuit . . . . . . . . . . 86

7.2 .1 Nonlinear model of the milling circuit . . . . . . . . . . . . 86

7.2 .2 Operating point and constraints . . . . . . . . . . . 90

7.2.3 Linearised model and input-output pairing selection of the milling circuit 91

7.2 .4 Input-output controllability analysis $\ldots \ldots \ldots . \ldots . \ldots 96$

7.2.5 Control performance specifications based on economic objectives . . . . . 100

7.3 Framework step 1: Base case identification . . . . . . . . . . . . 100

7.3.1 Proportional-integral-derivative controllers design . . . . . . . . . . 101

7.3.2 Functional performance of the proportional-integral-derivative controllers 101

7.3.3 Economic performance of the base case . . . . . . . . . . . . . . . 104

7.3.4 Performance bound . . . . . . . . . . . . . . . . 105

7.3.5 Decentralised controller based on decoupling . . . . . . . . . . 106

7.3.6 Motivation for using a nonlinear controller . . . . . . . . . . . . . . 112 
7.4 Framework step 2: Advanced controller design . . . . . . . . . . . . . . . 112

7.4.1 Control technologies used in milling circuits . . . . . . . . . . . . . 112

7.4.2 Nonlinear model predictive controller design . . . . . . . . . . . . . . 113

7.4.3 Performance of the nonlinear model predictive controller . . . . . . . . 116

7.4.4 Analysis of the origin of the advantage of the nonlinear controller . . . . 117

7.5 Framework step 3: Estimation of performance and initial economic assessment . 119

7.5.1 Estimate advanced process controller's performance . . . . . . . . . . 119

7.5.2 Initial economic assessment of the two controllers . . . . . . . . . . 120

7.6 Framework step 4: Implementation of the advanced controller . . . . . . . . . 120

7.7 Framework step 5: Calculation of performance and success assessment . . . . . 121

7.7.1 Statistical comparison of two controllers . . . . . . . . . . . . 121

7.7.2 Final assessment of the nonlinear model predictive controller and proportionalintegral-derivative controller . . . . . . . . . . . . . . . 125

7.8 Discussion . . . . . . . . . . . . . . . . . . . . . . . . . 128

7.9 Conclusion . . . . . . . . . . . . . . . . . . . . . . . 129

8 Investigation of the performance functions for the final stage of a bleach process 130

8.1 Introduction . . . . . . . . . . . . . . . . . . . 130

8.2 Process description . . . . . . . . . . . . . . . . . . 130

8.3 Performance function development for the $D_{2}$ stage $\ldots \ldots \ldots$. . . . . . . 131

8.4 Joint performance function . . . . . . . . . . . . . . . . . . . . 132

8.5 Conclusion . . . . . . . . . . . . . . . . . . . . . 133

9 Methodology for developing performance functions $\quad 134$

9.1 Introduction . . . . . . . . . . . . . . . . . . . . . . 134

9.2 Stage 1: Determine information required for performance function development . 135 
9.3 Stage 2: Gain required information on performance function development . . . . 136

9.3.1 Performance function-related information elicitation using survey research 136

9.3.2 Performance function-related information available in the literature . . . 137

9.3.3 Performance function-related information from plant tests . . . . . . . 137

9.4 Stage 3: Select suitable performance measures . . . . . . . . . . . . . . 137

9.5 Stage 4: Make suitable assumptions . . . . . . . . . . . . . 138

9.6 Stage 5: Determine performance functions . . . . . . . . . . . . . 139

9.7 Stage 6: Develop joint performance functions . . . . . . . . . . . . 140

9.8 Conclusion . . . . . . . . . . . . . . . . . . . . . . . . . . . 140

10 Conclusions $\quad 141$

10.1 Summary . . . . . . . . . . . . . . . . . . . . . 141

10.2 Assessment . . . . . . . . . . . . . . . . . . . . . . 143

10.3 Future work . . . . . . . . . . . . . . . . . . . . . 143

$\begin{array}{ll}\text { A Pairing investigation using relative gain array } & 154\end{array}$

A.1 Basic relative gain array theory . . . . . . . . . . . . . . . . 154

A.2 Relative gain array investigation for the milling circuit . . . . . . . . 155

$\begin{array}{ll}\text { B The derivation of minimum variance and relevant index } & 157\end{array}$

B.1 Feedback controller-invariance of minimum variance term . . . . . . . . . 157

B.2 The filtering and correlation algorithm for single-input single-output processes . 158

List of Figures 


\section{Chapter 1}

\section{Introduction}

\subsection{Motivation of economic performance assessment}

Control systems are widely applied to satisfy safety requirements and environmental regulations and to improve productivity. Maximising profits is, however, often regarded as the primary objective (Seborg et al., 2004). When considering the implementation of a control upgrade project, several measures must be considered: control performance, process performance and economic performance (Friedmann, 2006).

Control performance is a measure of how well the control system achieves its objective. This objective is usually expressed in terms of product quality and performance can be measured statistically and expressed as some function of deviation from target or variability. Among the commonly used functions are range, maximum error and standard error.

Process performance is a measure of how well the process achieves its objective. Various criteria can be used as measures. Most processes use energy to convert raw material into a product. Measures of process performance include production rate, expressed as units of product per unit time, and efficiency, expressed as units of product per unit of raw material and/or energy.

Improvement of control performance does not necessarily improve process performance. Sometimes it does, but often it only creates a platform for improved process performance. More often, process conditions or operating procedures have to be changed to take advantage of better control.

Economic performance is measured in financial terms. Industrial processes are usually operated to make money. Economic performance, like process performance, can be stated in terms of production or efficiency.

The relationship between process performance and economic performance is similar to that between control performance and process performance. Improved process performance does not necessarily guarantee that the process will make more money. Operating practices must often be changed to realise the potential benefits (Friedmann, 2006).

Overall, control improvement cannot guarantee an improvement in economic performance. How 
to assess and improve the economic performance of a controlled system is a topic deserving attention, which is the objective of 'economic performance assessment' (EPA) of a control system. EPA is receiving increasing attention in both academia and industry. It addresses the prediction of the benefit increase potential resulting from control upgrade projects and monitoring, calculation and improvement of economic performance when after-control projects are implemented. An assessment is therefore required by most manufacturing companies to weigh the benefits against the cost and risk of the new system (Martin, 2005), and is crucial to achieve world-class manufacturing (Brisk, 2004).

\subsection{A brief history of economic performance assessment}

EPA of control systems has been intertwined with advances in control technology. Fundamental developments in control technology usually require new approaches to investigate the corresponding benefit increase potential (Bauer and Craig, 2008). With the introduction of computers into control systems in the 1950s, the problem arose of whether the investment in control systems was worthwhile. Economic analysis in this period concentrated on straightforward physical and operational considerations, e.g. enhanced control improves throughput, leading to more income. Most manufacturing companies adopted new computer technology in the 1970s, often for the sake of technology itself. The energy crisis of the 1970s and 1980s required efficient processes and favoured the advances of control technology further. Explicit or implicit benefits from computer control were discussed, e.g. the availability of process running data. EPA during the 1970s and 1980s was mainly undertaken by control system suppliers such as Setpoint, Profimatics, Foxboro and Honeywell Inc. as justification for their products (Bauer and Craig, 2008). In the 1990s globalisation increased the pressure on manufacturing companies to decrease their costs and improve benefits. Only organisations with 'world class manufacturing' skills are likely to survive on the international market in the long term. There is more pressure today to produce as efficiently as possible and to use the most promising control techniques without wasting money on control failures. Owing to high design and implementation costs, EPA remains important for control technology suppliers and users.

\subsection{Overview of the current literature}

In this section, the origins of benefits from control systems are identified firstly and problems involved in EPA are listed. Then the literature on EPA is reviewed afterwards.

Approaches to EPA can be classified into two main categories. One is the benefit analysis method based on the Warren Centre case studies and their expansion (Marlin et al., 1987; Muske and Finegan, 2002; Martin, 2004). This category is referred to as the 'Warren family' in the context of this dissertation. The other is the method of using an integral to express a system's economic performance. This category is referred to as the 'integral family' in the context of this dissertation. There is a considerable difference between them. In the 'Warren family' the benefit from a control upgrade is proportional to the estimated process variable improvement; this notion often oversimplifies the relationship between process variables and their economic influence. However, in the 'integral family' the system's economic performance is not taken to be simply proportional to the process variable improvement. 


\subsubsection{Origins of benefits from control systems and problems involved in economic performance assessment}

In order to justify the cost associated with new control technologies introduced in a process, the benefits have to be justified in economic terms. This justification introduces what is referred to as EPA. EPA is crucial to further control technology developments and provides a business driver for process control.

Improved control benefits a process in that it enables moving the operating point of process variables in a more profitable direction (Marlin et al., 1987). Process variable variability reduction has also been discovered to yield considerable benefits (Craig and Henning, 2000; Latour, 1976, 1992; Stout and Cline, 1976).

EPA involves the following:

- Estimate improvement of operating point of crucial process variables.

- Develop performance functions (PFs) for the process.

- Estimate probability density functions (PDFs) for process variables.

- Estimate optimal operating points and optimal overall performance.

- Estimate the potential of performance improvement resulting from better control.

- Compare the economic performance resulting from several control technologies being considered and select the best one.

- Monitor the real time economic performance and optimise the performance.

\subsection{2 'Warren family'}

A general method of benefit prediction before control was produced by the Warren Centre case studies, which attracted the attention of both academia and industry (Marlin et al., 1987, 1991, 2001). This method begins with familiarisation with the processes, proceeds with an interview, a base case study, historical data analysis and developments of opportunities and ends with the calculation of potential benefits. A key step in this method is the estimation of process variable improvement. This method does not concern itself with an after-control system analysis, such as how to generate statistically valid data when comparing new and old controllers and drawing conclusions from a statistical point of view.

Three statistics-based approaches of process variable improvement estimation have been given (Martin et al., 1991; Martin, 2004; Muske and Finegan, 2002; Latour et al., 1986) to facilitate benefit calculation using the method proposed in Marlin et al. (1987). In these approaches the variable improvement is estimated against the prediction of variable standard deviation reduction. The frequently used reduction is $50 \%$ of standard deviation of the base case, whereas reductions such as 35\%,50-66\%, 85\% and 90\% are mentioned in other publications (Bauer et al., 2007). The reduction results from a control upgrade project and the intention is not to compare no-control with controlled cases. The approaches have the common limitation that the estimated variable improvement is determined subjectively and usually depends on the type of processes. The variable improvement can also be estimated from a simulation study.

Another method of estimating process variable improvement is given in Bawden (1993), which is based on a frequency domain analysis. In this method, disturbances that will influence the 
process are identified, and then the effect of the disturbances on the process is investigated using power spectral analysis of the disturbances and system transfer function. The process variable variance can subsequently be calculated according to the magnitude response of the loop transfer function. The calculated variance can then be used to derive the process variable improvement and then the overall improvement in system performance.

\subsection{3 'Integral family'}

There is a series of integral-based methods to consider the economic performance of a system (Latour, 1996, 2006; Zhou and Forbes, 2003; Mascio and Barton, 2001; Mascio, 2002). The basic idea is that system performance can be expressed as an integral of the product of the PFs and PDFs of a process's controlled variables (CVs). Economic performance of a controlled process is closely related to the fluctuation of CVs; PFs define either profits or costs when CVs move away from an optimal operating point. When the multivariate nature of a process's economic model is not considered, individual performance functions (IPFs) (PFs of different controlled variables) need to be developed. Otherwise, joint performance functions (JPFs) (PFs of dependent controlled variables) need to be developed. Although PFs can refer to IPFs or JPFs, when the multivariate nature is not stressed by default, PFs refer to IPFs. For an individual variable, performance functions could be linear, quadratic or cubic, etc. PDFs are often assumed to be normally distributed in earlier work on EPA (Latour et al., 1986; Zhou and Forbes, 2003).

PFs were first used to quantify the economic influence of control technology by Stout and Cline (1976), who also introduced modelling into the EPA process. Because the process variables vary over time, the corresponding performance changes as well. The overall performance is the average value of the performances related to the time series of the CVs. Quadratic and cubic functions are specifically discussed to show the impact of process variable variability reduction on the overall performance of the system. Although in Stout and Cline (1976) the overall performance is expressed as an expectation, it can be translated into an integral form since the definition of an expectation of a stochastic variable function is an integral of the product of the stochastic variable function and the relevant PDF.

Latour (1996) introduced an integral into EPA, where the PF is shaped like a CLIFFTENT. The economic impact of satisfying the specification and penalties for violating the specification are determined with this PF. Proper modelling of credits and penalties is conceived to be crucial for the overall assessment (Latour, 2006). CLIFFTENT also provides a heuristic way of determining the best operating point and best performance. This method is system-independent and is useful when the relation between process variables and the corresponding economic value of different variables are considered independently.

Although consensus has been reached that process variable variance reduction can lead to improved performance, the relation between them is not clear. A profit index has been given to this end (Bauer et al., 2007). Based on the assumption that each process variable follows a normal distribution, economic performance can be translated into a function of the standard deviation of the after-control-upgrade system. Then, the economic performance can be directly investigated through this function. The significance of this index is that it becomes clear how much of a reduction of variance from which value would improve economic performance significantly. The potential obtainable variance reduction from improved control is important 
and there are two kinds of regularly used methods to estimate this reduction (Bauer et al., 2007). One derives from experience in different industrial processes. The other is through a simulation study. Minimum variance provides a bound on the achievable performance improvement.

Control systems are considered as products and the relevant economic performance is regarded as products quality in Mascio and Barton (2001) and Mascio (2002), in which a measure of quality losses expressed in the form of an integral is used to quantify the system's performance in economic terms.

Zhou and Forbes (2003) propose a technique based on stochastic optimisation to obtain the optimal operating point and the optimal overall performance. The problems addressed include determining the optimal operating point and optimal performance of the current and upgraded system and performance bound. An optimisation technique is used for each scenario to obtain the respective solutions. The maximum attainable performance from a control upgrade is often not determined in current EPA, but is calculated by using the minimum variance controller in Zhou and Forbes (2003). Minimum variance performance is taken as a bound by most control loop performance assessment techniques (Huang and Shah, 1999; Jelali, 2006). There is a natural link between control loop performance assessment and EPA because they both consider process variable variance to be important. The derivation of a performance bound using minimum variance performance is also discussed (Muske, 2003), where process variables are considered independently.

\subsubsection{Other methods}

A general procedure is given to compare economic performance under the old and new controller when a control upgrade is implemented (Craig and Henning, 2000; Craig and Koch, 2003). The procedure includes four steps, namely experiment design and data generation, data analysis and hypothesis testing, monetary values calculation and economic project evaluation. Experiment design plays a key role because incorrect experiments can cause biased estimation of the quantity to be measured. Experiment design methods that can produce statistically valid information are discussed. Randomisation, replication and blocking are three basic laws for experiment design. Randomisation is to eliminate systematic errors and bias. Replication is to improve experiment precision. Blocking can be used to increase the precision of the experiment further. Completely randomised and randomised complete block designs are suggested.

Variance-based performance assessment could be translated to economic performance assessment for model predictive control systems (Xu et al., 2007). The objective of this method is to estimate the performance increase potential with improved control. The assumption is that the objective function of the model predictive controller is quadratic and the constraints of the process variables are linear. The algorithms use linear matrix inequalities to obtain the economic performance of model predictive control system based on the variable variance. Routine operating data and the process steady-state gain matrix are inputs of the algorithms. The algorithms are programmed to be a part of a multivariate control performance assessment toolbox (Huang et al., 2006).

A benchmark is provided for evaluating control systems' economic performance (Brisk and Blackhall, 1995). The performance of the systems could be investigated on the basis of ten aspects; each aspect is scored and then added to the overall performance index. This index 
could be used to judge the level of system performance. Based on this index, a statistical method with two critical indices is introduced to identify the scope of improvement derived from the control upgrade (Lant and Steffens, 1998). These two methods can only provide rough guidelines of the current performance and improvement potential.

\subsubsection{A framework of economic performance assessment}

A framework of EPA is proposed (Bauer and Craig, 2008), based on the existing economic assessment technologies. It involves base case identification, controller design, performance estimation, controller implementation, performance calculation, maintenance of the system and recalculation of performance. Guidelines are given to perform each step. The framework provides a comprehensive EPA tool. In steps 1, 3, 5 and 7 the performance of the system needs to be estimated or calculated, based on PFs and PDFs. The limitation of the framework is the lack of a systematic method for developing PFs.

\subsection{Research objectives and approaches}

\subsubsection{Research objectives}

PFs play an important role in EPA, which is shown in the framework proposed in Bauer and Craig (2008). Although a number of achievements have been reached, there is no systematic way of developing PFs (Zhou and Forbes, 2003). This restrains further research into EPA because without well-established PFs, economic assessment cannot continue smoothly and therefore cannot effectively support decision-making for management. The development of PFs is a bottleneck in the further investigation of EPA.

The first objective of this research is to establish a methodology of developing PFs. Furthermore, the multivariate nature of processes will be investigated in order to derive some ideas on helping to establish joint PFs based on individual PFs. The third objective will be to conduct a case study of EPA of a grinding mill circuit using the framework proposed in Bauer and Craig (2008) in order to show how to perform an EPA in a formal way. Another objective is to determine the current practice and guidelines on how the grinding circuits are controlled and how the functional/economic performance is conducted for these circuits in practice.

\subsubsection{Research approaches}

The methodology of developing PFs is established based on in-depth investigations of developing PFs for a continuous process (grinding mill circuit) and a batch process (electric arc furnace). In addition, the development of PFs for a stage of a bleach process is also examined and the thrusts that were used in the development are extracted.

A case study on developing PFs of a grinding mill circuit, an energy- and cost-intensive process in the mineral processing industry, is conducted first. The development of PFs of the grind- 
ing mills starts from a deep understanding of the mill circuit's operation described in some monographs and publications on the economics and control of the grinding circuits. Then a web-based survey study is conducted and, as a part of the survey study, the economic influence of the frequently used controlled variables of grinding circuits is investigated and instructive information on PFs is obtained from the survey results. Moreover, a comprehensive literature study is conducted and the consistency of the relation between the particle size distribution (PSD) of the grinding circuit's product and mineral recovery of the downstream flotation circuits is revealed and used as the basis of the PF of the PSD. An interview with an experienced engineer in grinding control also provides illustrating information on PFs. Then the PFs of the grinding circuit are developed based on the results of the web-based survey study and the literature study and interview.

Individual PFs of an electric arc furnace (EAF) were developed in Oosthuizen et al. (2004) and used to evaluate the economic performance of a model predictive controller for the furnace. The development of the PFs is reviewed critically and ideas used are abstracted. Furthermore, the multivariate nature of the furnace is investigated and the JPFs of the furnace are constructed. The development of the PFs for a $D_{2}$ stage of a bleach process is examined and the ideas used are extracted. The methodology of establishing PFs is then proposed by integrating the ideas used for developing PFs of the processes mentioned above.

An economic assessment of three controllers, i.e. single-loop proportional-integral-derivative (PID) controllers, a decentralised controller and a nonlinear model predictive controller (NMPC), for the grinding circuit is undertaken using the PFs developed for the following two reasons. One is to illustrate the economic advantage of the NMPC controller using a carefully designed statistical experiment and the other is to show how to conduct the economic evaluation of controllers systematically.

\subsection{Contributions}

The contributions of this study are as follows:

1. PFs of grinding mill circuits are developed.

2. JPFs of an EAF are developed based on the individual PFs.

3. A methodology of developing PFs is proposed.

4. Current practice of control and functional/economic performance assessment on grinding mill circuits is determined through a web-based survey study.

5. A case study of EPA on an NMPC, a decentralised controller and three single-loop PID controllers of a grinding mill circuit is conducted. This case study firstly shows the significant role of PFs in a systematic EPA. It also illustrates that the NMPC controller yields statistically better economic performance over PID controllers. Furthermore, it shows how to conduct an EPA of a control system systematically.

The contents of this thesis have been published as follows: 
Wei, D. and Craig, I. K. (2009a). Development of performance functions for economic performance assessment of process control systems, Presented at IEEE Africon 2009.

Wei, D. and Craig, I. K. (2009b). Economic performance assessment of two ROM ore milling circuit controllers, Minerals Engineering. 22: 826-839.

Wei, D. and Craig, I. K. (2009c). Grinding mill circuits - A survey of control and economic concerns, International Journal of Mineral Processing 90: 56-66.

Wei, D. and Craig, I. K. (2008). Grinding mill circuits - A survey of control and economic concerns, Proceedings of the 17th IFAC World Congress, Korea, pp. 1000-1005.

Wei, D., Craig, I. K. and Bauer, M. (2007). Multivariate economic performance assessment of an MPC controlled electric arc furnace, ISA Transactions 46(3): 429-436.

Wei, D., Craig, I. K. and Bauer, M. (2006). Multivariate economic performance assessment of an electric arc furnace, Proceedings of the SACAC Control Conference, Durban, South Africa, pp. 1-6.

\subsection{Organisation of thesis}

The chapters in this thesis are organised as described below. Some preliminary economic analysis knowledge is given in Chapter 2. A framework of EPA of control systems is reviewed critically in Chapter 3. The development of IPFs and JPFs for an EAF is discussed in Chapter 4. The $\mathrm{PF}$ development of a stage of a bleach plant is reviewed subsequently in Chapter 8 . The current practice of process control and (functional/economic) performance assessment of grinding mill circuits is reported in Chapter 5. The development of PFs for grinding mill circuits is elaborated on in Chapter 6. An EPA of three run-of-mine (ROM) ore milling circuit controllers is given in Chapter 7. A methodology of developing PFs is then proposed in Chapter 9. Conclusions are drawn in Chapter 10. 


\section{Chapter 2}

\section{Preliminary economic analysis}

For any process, before the PFs are developed, preliminary economic analysis is required to work as a starting point of the PF development, which is discussed in this chapter. Firstly, process operation and control understanding is dealt with. The identification of possible benefit is then discussed.

\subsection{Process operation and control understanding}

In order to derive the PFs of a process, the first step required is to gain in-depth understanding of the process operation. The primary objective of understanding the process is to establish a good basis for obtaining information on process economics. The required information includes:

- Production objectives.

- Process description.

- Feedstock list used for production.

- Energy used for production.

- Possible disturbances.

- Measurement instrumentation used.

Exploration of the control system used may also reveal instructive information. Specifically, the following information needs to be obtained:

- Manipulated/controlled variables being selected.

- Reason for selecting these manipulated/controlled variables.

- Considerations when determining specifications for the CVs.

- Compensative action when the CVs deviate from their specifications.

- Economic impact when the CVs violate their specifications.

- Final control elements used.

This information may contribute to the establishment of PFs. 
Information on process economics is the most important for the development of PFs. For each CV of the process, the economic impact can always be classified as two types: cost and benefit. Cost or benefit associated with a control project can usually be classified into several fixed categories. Each CV needs to be checked against the benefit list and variable operating cost profile discussed below to further understand process economics and the relationship between the CVs and cost/benefit. In this section, the possible benefits resulting from a control improvement are discussed. They are elaborated on in Friedmann (2006) and Bauer and Craig (2008), as summarised below.

\subsubsection{Higher throughput}

Not all control improvements can be used to raise productive capacity. Many continuous processes are physically constrained, usually by the capacity of some transport mechanism e.g. pump or conveyor belt, and better control cannot remove the limitation. Generally, there is a trade-off between production throughput and quality (Bauer and Craig, 2008). Sometimes, production throughput has higher priority for the sake of the extraneous economic environment. An increase in throughput will improve the system's economic performance, providing a good opportunity to make profit. Batch processes are good candidates to gain higher output. Speeding up either batch time or time between batches will raise production. An improvement that allows omission of a step or steps in the process can result in a larger increase in throughput. Installation of on-line analysers and feedback control of composition often allows elimination of laboratory analyses and subsequent product blending. Serial multi-product processes are also good prospects. Better control can reduce transition time from one set of conditions to another, thus reducing waste and increasing production.

Even if throughput can be significantly increased by better control, the result will not be useful unless the additional product from the plant can be sold. If demand exceeds capacity, then more product can be sold directly. If the company has multiple plants making the same product, then the increased production at Plant A may be used to replace higher-cost production at Plant B.

Some products (e.g. fertiliser and toys) are very seasonal, so production during most of the year is for inventory. An increase in plant capacity can make money for these products by allowing production to increase in season, thus decreasing the requirement for working capital.

\subsubsection{Lower utility costs}

Many processes are energy-intensive. Fuel, steam and electricity costs form an appreciable part of operating expenses. These processes, including power generation, metal refining and petrochemical production, are good candidates for utility cost savings (Bauer and Craig, 2008). Especially good prospects are processes with high and rapidly changing energy requirements. Control improvements that level the energy load can be very attractive. If the energy is supplied by coal, oil, or natural gas, load levelling will reduce transitions and improve combustion 
efficiency. If electric energy is used, load levelling will reduce peak demand, for which power companies make a separate substantial charge.

If energy is supplied from sources with differing costs, automatic load allocation can produce savings. Load allocation selects the lowest cost mix of sources that will satisfy the demand and continually adjusts this mix as the load changes. Electric utilities and their suppliers have developed highly sophisticated load allocators that cover entire regions. Simpler programs are often useful for industrial applications. It should be noted that both load levelling and load allocations require some excess process capacity. If all equipment is being run full out to meet production requirements, neither method is applicable.

\subsubsection{Better yield}

Any processes with substantial raw material costs and yields significantly below $100 \%$ will benefit from yield improvement (Bauer and Craig, 2008). Among the likely candidates are processes that make high purity material. A high purity requirement often requires discarding considerable amounts of product along with un-reacted raw material or byproducts. An example of a distillation column shows how better control, allowing the final product set point to be moved closer to specification, can produce a significant increase in yield.

Another process characteristic that indicates a good possibility of yield improvement is frequent grade or product changes. The material produced during these changes is likely to be offspecification with respect to both products, so any control change that stabilises the process more quickly will improve yield.

Many of the control improvements that can be used to improve yield can also be used to increase production if the process is not otherwise limited. A choice between objectives will depend on the economic situation and may change during the life of an installation. (For instance, a plant may anticipate running at full capacity for two years, until competition comes on line, and thereafter at a reduced rate. In this case, the benefits would be increased production during the first two years and increased yield afterwards.)

\subsubsection{Fewer unwanted byproducts}

Increasingly stringent restrictions on waste disposal have made this cost-saving area much more important. Process viability can now quite easily be dependent upon getting rid of byproducts at reasonable cost. Obviously, those processes that turn out variable quantities of byproducts with disposal problems are the best candidates in this case. Control improvements that enable a plant to avoid installing waste treatment facilities are especially attractive. (For instance, a $p H$ control system that keeps effluent $p H$ in the 6.0-9.0 range might allow a plant to defer installation of a holding and treatment lagoon for waste water.)

A control system that deals with potentially toxic waste but does not eliminate toxicity is valuable. Sensing the presence of a toxic component and segregating the material containing it can limit the amount that must be handled with special precautions. Perhaps only a drum or two will need to be sent to a hazardous dump rather than a truckload. 


\subsubsection{Less labour}

The general public perceives reduced direct labour as the major cost-saving deriving from automation. As far as process industries are concerned, this is rarely correct. Direct labour is a relatively small fraction of the total cost for almost all continuous processes and many batch processes.

It is fairly easy to identify a process for which automation can reduce direct labour. The key is the number of operator stations. Reduction of indirect labour is a more likely significant benefit. Recording-keeping can often be incorporated into a control system that already collects all the information needed for monitoring purposes. The same information can readily be totalled and used to produce reports for management and regulatory agencies, replacing clerical technical labour. Reports can sometimes be eliminated completely by integrating the control system into a computer-integrated manufacturing network.

\subsubsection{Better quality}

A particular control improvement can often be used in different ways to produce any of several benefits (e.g. more production, lower cost or better product). Added production or cost savings are usually, but not always, chosen rather than better quality. Improving the product can be a good choice if there is a strong relationship between quality and product price (Bauer and Craig, 2008).

The viability of this option may depend on breakpoints for price changes. Product price tends to be a discontinuous function of quality with fixed break points. An improvement in quality to just under the next break point is worth nothing, while a slight additional increase across the break point produces a large return. (In the example shown in Section 3.5 of Friedmann (2006), decreasing the percentage of impurity from point 1 to 2 actually loses money, since the operating cost increases while the product is still Grade B, so the selling price cannot be increased. Decreasing the percentage of impurity from point 2 to 3 is profitable, as the product is now Grade A with a higher selling price. The increase in operating cost is more than offset by increased revenue.)

It should be noted that profits from quality improvement may depend on market conditions. Price differences among product grades can grow or shrink. Benefits from quality improvement are probably less market-dependent than those from production increases and more dependent than those from cost savings.

\subsubsection{Quantifiable benefits from reducing pollution}

Pollution-reduction projects may be started by non-economic imperatives. This does not necessarily mean that there are no quantifiable benefits from such projects. These benefits include obvious incentives for avoiding regulatory penalties, which may take various expensive forms, including fines and mandatory limits on production. Some benefits may be less apparent. Plant capacity may be increased if it has been limited by an environmental constraint. 


\subsubsection{Quantifiable benefits from improving safety}

Safety improvement projects may also be started for non-economic reasons. These projects, in addition to increasing safety, may have other quantifiable benefits. Some of these benefits are similar to those from reducing pollution, especially avoidance of regulatory penalties. Others may stem from damage prevention. Sudden equipment failures are both dangerous and expensive. The likelihood of such failures can be limited by the installation of equipment monitors that can detect when critical components need maintenance. Utilities, for which downtime can be particularly expensive, are among the most enthusiastic users of predictive maintenance.

\subsection{Conclusion}

In this chapter, preliminary economic analysis, which is intended to work as a start point for the PF development for various processes, is discussed. An in-depth insight of process operation and control is required firstly. Then it is necessary to figure out the major possible benefits that may result from the improvement in the variations of process variables. 


\section{Chapter 3}

\section{Review of a framework on economic performance assessment}

\subsection{Introduction}

Methods for EPA of control systems used in the past decades were reviewed and incorporated into a framework in Bauer and Craig (2008), as shown in Fig. 3.1. This framework consists of seven engineering steps and a managerial process, and will be used to assess the economic performance of three controllers of a run of mine (ROM) ore milling circuit in Chapter 7 . In this chapter, the framework is reviewed critically to facilitate the assessment as will be conducted in Chapter 7. Steps 1 to 3 are undertaken before the controller is implemented (step 4), with the calculation of performance and success assessment (step 5) taking place immediately after implementation. Maintenance (step 6) and long-term assessment (step 7) will not be dealt with in this chapter. PFs play a crucial role in this framework and are used in steps 1,3 and 5 (Bauer et al., 2007). Furthermore, owing to how this framework was shaped, this chapter can also be regarded as a supplementary part of the literature review of EPA of control systems.

\subsection{Step 1: Base case identification}

\subsubsection{Collect base case data of controlled variables of interest}

When considering a control upgrade project, the first step required is to collect the base case data that can represent the typical process operation with the current control system. The controller in place, if applicable, is supposed to be well tuned. This step needs a deep understanding of the process considered, e.g. the process dynamics, economics, operating conditions, operating constraints and so on. Determining the base case operation also requires an in-depth analysis of historical process data, comprising control room data sheets or computer-logged data, laboratory data sheets, operating targets and operator logs (Marlin et al., 1991). Data should not be used for periods when the process is operating in unusual situations, e.g. start-up and shut-down, or when an atypical equipment limitation appears. Appropriate CVs should be focused on, to enable the extraction of more information in the following steps. 


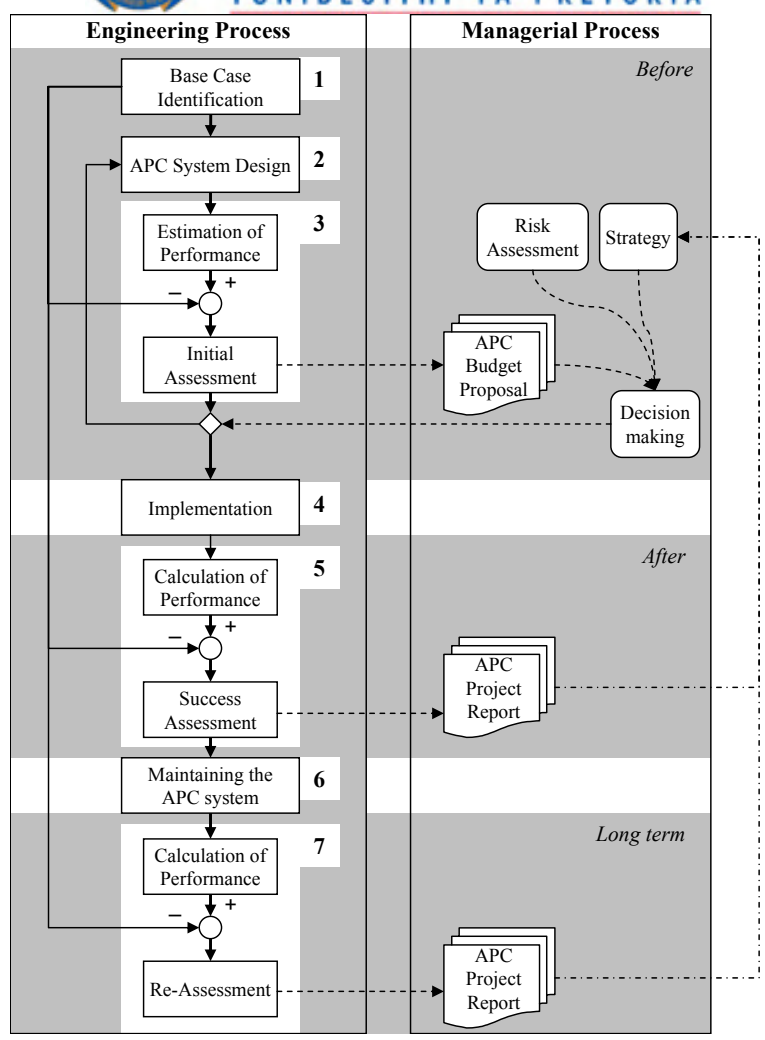

Figure 3.1: The framework of EPA (Bauer and Craig, 2008)

Critical product qualities, important manipulated variables, material and energy balance parameters and constraint variables are recommended to be analysed in Latour et al. (1986). Note that in this thesis the economic relations in a process are regarded as related to mainly CVs. The economic impact of manipulated variables is not considered. The reason for focusing on the constraint variables is that the economic performance improvement in a process is often related to constraints of process variables. The reason for selecting critical product qualities and material and energy balance parameters is that they are often tightly related to the crucial monetary values.

\subsubsection{Estimate the base case's probability density functions}

Time series of control loop outputs are usually assumed to be stationary and ergodic in the control loop performance assessment literature (Choudhury et al., 2004), which holds in this thesis as well. This assumption is reasonable, given that the control loop is working properly. Time series are usually assumed to follow Gaussian distributions when generated by a linear source. As most process control loops are approximately linear in a specific operating region, the time series of a CV is usually assumed to be Gaussian (Zhou and Forbes, 2003). Furthermore, the non-Gaussianity of a time series is regarded as an indication that the loop may be working poorly (Thornhill and Horch, 2007; Choudhury et al., 2004). For a one-dimensional Gaussian distribution, $\mu$ and $\sigma$ are enough to decide the PDF for single variables, as follows:

$$
f(y)=\frac{1}{\sqrt{2 \pi} \sigma_{y}} \exp \left\{-\frac{\left(y-\mu_{y}\right)^{2}}{2 \sigma_{y}^{2}}\right\}
$$

where $y$ is a CV. $\mu_{\text {base }}$ and $\sigma_{\text {base }}$ are used to determine the PDFs of the base case. 
If the multivariate nature of the process economic model is considered, the joint probability density functions (JPDFs) need to be used. For instance, for two CVs $x$ and $y$, the Gaussian JPDF is

$$
f(x, y)=\frac{1}{2 \pi \sigma_{x} \sigma_{y} \sqrt{1-\rho^{2}}} \exp \left\{-\left[\left(\frac{x-\mu_{x}}{\sigma_{x}}\right)^{2}-2 \rho\left(\frac{x-\mu_{x}}{\sigma_{x}}\right)\left(\frac{y-\mu_{y}}{\sigma_{y}}\right)+\left(\frac{y-\mu_{y}}{\sigma_{y}}\right)^{2}\right] / 2\left(1-\rho^{2}\right)\right\}
$$

where $\rho$ is the correlation coefficient of two random variables defined by

$$
\rho=\frac{\sigma_{x y}}{\sigma_{x} \sigma_{y}}
$$

where $\sigma_{x y}$ is the covariance of $x$ and $y$, given as

$$
\sigma_{x y}=E\left[\left(x-\mu_{x}\right)\left(y-\mu_{y}\right)\right]
$$

where 'E' means the expectation.

\subsubsection{Develop performance functions $(v(y))$}

Instead of mapping the economic influence of the CV improvement due to improved control as a constant value, as in Marlin et al. (1987) and Martin (2004), PFs define the profit or loss when CVs move away from the optimum operating point. PFs reflect the economic model of profit/loss vs CVs. PFs provide a useful tool for estimating economic performance of various controllers and play a crucial role in EPA (Bauer et al., 2007; Friedmann, 2006; Martin, 2005). There are several frequently used PFs in process industries, i.e. a linear PF with a constraint, a CLIFFTENT and a quadratic PF, as given in Fig. 3.2.

A linear PF with a constraint reflects a common phenomenon of profit, which changes linearly with the move of a process variable until a threshold. Once this is transcended the product will become waste and profit becomes zero. This PF has been discussed for a fuel oil blending process in Latour (1976), where the profit increases linearly with the quantity of sulphur content. Once the content breaks the limit, environmental regulations will be violated and the product will be reckoned as waste. This type of PFs is also extended for cases where over-specification of material vs. a sliding penalty on the selling price is considered, resulting in more realistic linear PFs. It is important that linear PFs can be used to establish complex piecewise linear PFs.

The use of the CLIFFTENT PF was initiated by Latour (1996) and it is examined in Zhou (2002) to reveal the relation of profitability and brightness of pulp in a bleach process. It is named after the shape of the function. The CLIFFTENT PF can be expressed by the sum of two linear constrained PFs. The constraint here refers to where the maximum benefits are realised for zero variance. For nonzero variance, the optimal operating point needs to be estimated based on the predicted variable variance.

For a quadratic PF, the maximum benefit is fulfilled if the process variable lies at an optimal operating point; the benefit diminishes when the variable deviates from the operating point. The benefit vs. variable can be approximated by a quadratic function with second-order polynomial fit. It is referred to as the curvature rule in Martin et al. (1991) and used in the economic assessment of an ammonia production process to reflect the relation between throughput and composition ratio in Stout and Cline (1976). For a quadratic PF, the reduction of process 


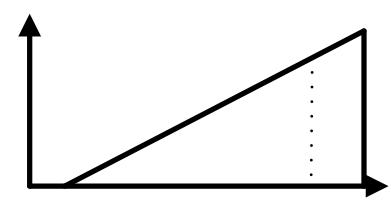

(a) Linear constrained PF

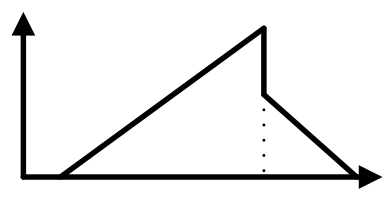

(b) CLIFFTENT PF

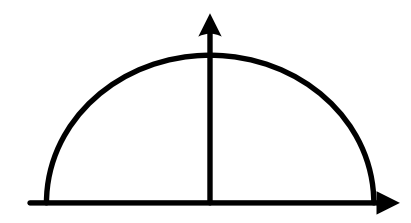

(c) Quadratic PF

Figure 3.2: Frequently used PFs

variable variance will centre the process data to the operating point and the operating point will not change with the change of process variable variance.

When the economic impact of CVs is considered separately, the IPFs need to be developed. However, when the economic model's multivariate nature is considered, i.e. the interaction of the economic impact of CVs is considered, JPFs are developed. That is the case in Wei et al. (2007) and Zhou and Forbes (2003). A decision needs to be made at the beginning of EPA whether to develop JPFs or IPFs, which has a significant influence on some decisions that need to be made later, e.g. how to determine the optimal operating point or how to estimate/calculate the economic performance index.

PFs are often difficult to derive, differ from process to process and even change within a process with changes in extraneous economic conditions. Comprehensive insight into the operation and economics of the process and sales agreements is required. It is to be hoped that skilled engineers have some perception of the economics of the process being investigated. The insight can be used to construct PFs when using carefully considered questioning (Oosthuizen et al., 2004; Wei and Craig, 2009b). PFs can also be built based on plant experiments (Craig et al., 1992b) or through an analysis of product value vs production costs (Zhou and Forbes, 2003). The development of PFs is the focus of this thesis and will be discussed in depth in Chapters 4 and 6.

\subsubsection{Calculate the economic performance index of the base case $\left(\Psi_{\text {base }}\right)$}

The economic performance of a control system can be expressed as a monetary value, referred to as the economic performance index (EPI). The EPI $\left(\Psi_{y}\right)$ associated with a CV $(y)$ can be estimated in two ways: continuous and discrete calculation methods.

\subsubsection{Continuous calculation method}

For the continuous method, an EPI is estimated by integrating the product of the relevant PF $(v(y))$ and $\operatorname{PDF}(f(y))$, as follows:

$$
\Psi_{y}=\int_{-\infty}^{\infty} v(y) f(y) d y
$$

The EPI decreases monotonically with the variable variance for the three PFs mentioned in Section. 3.2.3. The continuous calculation method is increasingly used (Latour, 1996, 2006; 
Zhou and Forbes, 2003; Mascio and Barton, 2001; Bauer and Craig, 2008) because of increased accuracy in estimating the economic performance compared to the methods used in Martin et al. (1991); Martin (2004); Muske and Finegan (2002). For instance, in the method proposed in Marlin et al. (1991), the economic impact of a CV is taken as a linear function of the CV, which may not hold in many cases; this method oversimplifies the relation between economic influence and the CVs and will therefore result in less accurate estimation of economic performance. In Section 3.5 of Friedmann (2006), a PF of the proportion of distillate A is given, as shown in Fig. 3.3, with the benefit expressed in terms of the product yield. The seemingly insignificant change in composition has a large effect on yield and the PF is definitely not a linear function.

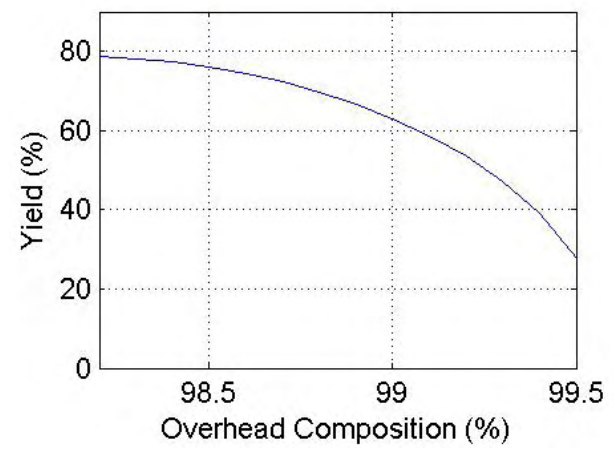

Figure 3.3: PF of distillate composition (Friedmann, 2006)

An EPI of a variable is a function of a PF and PDF. $\mu$ (as in Subsection 3.2.2) of a time series refers to the operating point in this context, not necessarily the setpoint of the $\mathrm{CV}$; as only when a controller can track the setpoint over the long term, the setpoint is equivalent to the operating point. According to the rationale of the method using the convolution of PDFs and PFs to derive EPI, the time correlation does not matter. For instance, a given process variable exhibiting, in two different control situations, the same PDF but different autocorrelation functions will produce the same EPI. Note that a good controller is supposed to have satisfactory performance in terms of setpoint tracking and disturbance rejection, but owing to significant and uncontrollable disturbances, the setpoint may be different from the operating point.

The EPI is then a function of the operating point and standard deviation $(\sigma)$ of the time series of the $\mathrm{CV}$ being considered. It can further be regarded as a function of only $\sigma$ where the operating point is decided based on the standard deviation, which will be discussed later. However for the base case, the EPI can be calculated based on the PF and the PDF of the CV examined.

\subsubsection{Discrete calculation method}

An alternative manner of estimating the EPI is discrete summation, for which $\mathrm{N}$ samples of a process variable are used and the EPI can be calculated as

$$
\Psi(y)=\frac{1}{N} \sum_{i=1}^{N} v\left(y_{i}\right)
$$




\subsubsection{Comparison of continuous and discrete methods}

Both continuous and discrete calculation methods can be used to calculate the EPI, when the time series of CVs are available (Akande et al., 2009). However, the advantage of the continuous method over the discrete method is that it can be used to estimate the EPI using estimated $\mu$ and $\sigma$ when the time series is not available and, in some cases, to estimate the optimal operating point given an estimated variable variance. The advantage of the discrete method is that it can provide performance estimation/calculation results without consideration of the PDFs.

\subsubsection{Overall economic performance index derivation based on individual eco- nomic performance indices}

\subsection{Univariate derivation}

When the multivariate nature of the PF model is not considered, the overall EPI of the whole system is the sum of the EPIs associated with individual CVs of interest, as in Oosthuizen et al. (2004). If there are $p \mathrm{CVs}$, the overall EPI is therefore

$$
\Psi=\sum_{i=1}^{p} \Psi\left(y_{i}\right)
$$

The EPI of the base case $\left(\Psi_{\text {base }}\right)$ can be estimated by either using the continuous or discrete methods, based on the data collected in step 1.1 and PFs obtained in step 1.3.

\subsection{Multivariate derivation}

However, when the multivariate nature is contemplated, the EPI of dependent variables will be derived by integrating the JPF and JPDF (Wei et al., 2007). The multivariate derivation method will lead to more accurate results (Zhou, 2002; Zhou and Forbes, 2003). Assume there are $p$ CVs, the first and second variables are dependent and the third and fourth variables are also dependent, but all other variables are independent, then the overall EPI is

$$
\Psi=\Psi\left(y_{1} y_{2}\right)+\Psi\left(y_{3} y_{4}\right)+\Psi\left(y_{5}\right)+\Psi\left(y_{6}\right)+\ldots+\Psi\left(y_{p}\right)
$$

\subsubsection{Estimate the minimum variance $\left(\sigma_{m v}^{2}\right)$}

The reduction of CV variance $\left(\sigma^{2}\right)$ usually results in the improvement of the economic performance of a control system. However, for a process with a given operating condition, there is a threshold for the reduction of variance, referred to as minimum variance $\left(\sigma_{m v}^{2}\right)$ in the field of 'control loop performance assessment' that was pioneered by Harris (1989) and has been intensively researched (Harris et al., 1996; Huang and Shah, 1999; Huang et al., 2005; Jelali, 2006). The reason for $\sigma_{m v}^{2}$ being the minimum variance is that a controller cannot take any compensative action to reject disturbances to reduce $\sigma^{2}$ of a CV below $\sigma_{m v}^{2}$ (Huang and Shah, 1999). The controller that can provide $\sigma_{m v}^{2}$ is known as a minimum variance controller. The minimum variance is often used as a benchmark to evaluate whether a controller provides satisfactory performance. Minimum variance can usually not be realised, but provides a bound on controller performance. 
A number of algorithms are proposed to estimate $\sigma_{m v}^{2}$, often with a predetermined structure of the controlled process, such as feedback control, feed-forward control, feedback plus feedforward control or model predictive control, etc. The algorithms are usually based on the given structure. For instance, the derivation of $\sigma_{m v}^{2}$ for a feedback control scheme is given in Appendix B using a filtering and correlation algorithm given in Huang and Shah (1999). An overview of control performance assessment technology and industrial applications is given in Jelali (2006), in which various algorithms are highlighted and compared, and respective advantages and disadvantages are featured. Note that minimum variance controllers are seldom implemented in practice because of their aggressive control actions and limitation on robustness (Huang and Shah, 1999). For a given operating condition the economic performance bound can be related to $\sigma_{m v}^{2}$, as $\sigma_{m v}^{2}$ can be used to determine the optimal operating point $\left(\mu_{m v}\right)$ of the minimum variance controller.

\subsubsection{Determine the operating point $\left(\mu_{m v}\right)$ of CVs for $\sigma_{m v}^{2}$}

The most favourable benefits often occur when there are constraints on process variables. Fluctuation of CVs often takes place as a result of e.g. process disturbances. The fluctuation can take the CVs past the constraints, which could be very dangerous and expensive. For instance for an electric arc furnace in the steel-making industry, when the steel is tapped, if the percentage of carbon exceeds an upper limit, the melt may be scrapped, implying that the melt cost would be more than double (Oosthuizen, 2001). Also for the electric arc furnace, a positive off-gas temperature at tapping time will result in the emission of dust and fumes into the workshop, causing a safety hazard. The operating point often needs to be increased to new values near constraints for economic reasons. There is a generic trade-off, as the closer the operating point is to the constraints, the more benefits are often achieved, but once the constraints are violated, the economic impact could be severely negative. The selection of the operating point is a challenging issue. There are mainly two kinds of methods for obtaining the optimal operating point based on the variable variance. The first class is statistics-based methods and the second is optimisation-based. In addition, two other rules can also be used for this purpose. The optimal operating point sometimes remains constant whatever the variance is, if e.g. the PF is parabolic.

\subsubsection{Three statistic-based methods to derive the optimal operating point}

Three frequently used statistics-based rules include the 'same limit rule', the 'same percentage rule' and the 'final percentage rule' (Martin et al., 1991; Muske, 2003). With these rules, the standard deviation of a CV $\left(\sigma_{e s t i}\right)$, after the control-upgrade project has been done, is assumed to be a fixed percentage of $\sigma_{\text {base }}\left(\sigma_{\text {esti }}=k \sigma_{\text {base }}\right)$, as will be discussed in step 1.9. $\Delta_{\mu}$ refers to the change of the operating point. $l$ represents the limit (constraint). The time series of a CV is assumed to follow a Gaussian distribution, and the probability that this variable is greater than a given value $\left(x_{l}\right)$ is noted as $\alpha$ (significance level).

Same limit rule When a large percentage of the base case data violates the limit, the limit must be neglected and a new limit needs to be selected for both before- and after-control upgrade data. The limit should be selected such that only $5 \%$ of the base case data exceed it. 
Because

$$
\frac{l-\mu_{\text {base }}}{\sigma_{\text {base }}}=\frac{l-\mu_{\text {esti }}}{k \sigma_{\text {base }}}=x_{l}
$$

so

$$
\Delta \mu=\mu_{e s t i}-\mu_{\text {base }}=x_{l}(1-k) \sigma_{\text {base }}
$$

specifically when $\alpha$ is $0.05, x_{l}$ is 1.65 . Therefore

$$
\Delta_{\mu}=0.825 \sigma_{\text {base }}
$$

Same percentage rule When a reasonable percentage of the before-control data hits the limit, the after-control data can be allowed to hit the constraint by the same percentage. Therefore

$$
\Delta_{\mu}=\frac{l-\mu_{\text {base }}}{2}
$$

Final percentage rule When not much before-control data hit the limit, the new operating point is set up such that $5 \%$ of after-control data hit the limit. Therefore

$$
\Delta_{\mu}=l-\left(\mu_{\text {base }}+1.65 k \sigma_{\text {base }}\right)
$$

\subsubsection{An optimisation-based method to derive the optimal operating point}

As given in Section 3.2.4, when the continuous method is used to obtain an EPI, and when the PF and variable variance are already available, the EPI will be determined only by $\mu$, which is the operating point (or CV setpoint) that needs to be determined. Accordingly, the operating point derivation problem is converted to an optimisation-based one. This heuristic method was pioneered in Latour (1996) and discovered to be valuable in Zhou and Forbes (2003) and Mcmahon (2004). Furthermore, this method is used in Bauer et al. (2007) to obtain the optimal operating point and the relevant EPI for the three frequently used PFs discussed in Section 3.2.3. Then, $\mu_{m v}$ can be derived using this method based on $\sigma_{m v}$.

\subsubsection{Two other rules}

Achievable operation rule If a considerable percentage of data stay around the limit, the 'achievable operation rule' may be used, for which the limit is set up as the new operating point (Martin, 2004), therefore

$$
\Delta_{\mu}=l-\mu_{\text {base }}
$$

Best operator rule This is based on the assumption that the advanced controller can consistently perform as well as the best operator under the same conditions. Room for improvement depends on the difference between the average operating point and best operating point. Sometimes even greater improvement can be claimed. This rule has been found useful for estimating savings generated by controller performance improvement (Marlin et al., 1991). Therefore

$$
\Delta_{\mu}=x_{\text {best }}-\mu_{\text {base }}
$$




\subsubsection{Unchanging optimal operating point with varying variance}

The operating point, however, sometimes does not change with a reduction of variable variance. For instance, if the PF is quadratic and the optimal operating point is the value that makes the quadratic function culminate, and there is no constraint around this optimal value, then the optimal operating point remains unchanged regardless of how much the variable variance will be reduced. This is the case for the 'curvature rule' in Martin (2004).

\subsubsection{Calculate the maximum achievable performance $\left(\Psi_{m v}\right)$}

The EPI of the minimum variance controller $\left(\Psi_{m v}\right)$ can be derived based on $v(y)$ (developed in step 1.3), the optimal operating point (obtained in step 1.6) and $\sigma_{m v}$ (obtained in step 1.5). It is assumed here that the setpoint can be tracked well over the long term. $\Psi_{m v}$ can then serve as the performance bound to which the EPI of various cases can be compared to see how much improvement may be achieved by control efforts.

\subsection{Step 2: Advanced controller design}

Once the need for a new control system has been determined, the next step is the design of the new controller. Various control technologies can be selected, depending on the situation in the process. Control technologies used in the process industries are investigated in a webbased survey (Bauer and Craig, 2008) and the results given in Fig. 3.4. It can be seen that model predictive control is popularly used. Furthermore, constraint control and split-range control are also used frequently in the continuous processing industries. Computational artificial intelligence techniques, e.g. neural networks and fuzzy logic, are used less frequently.

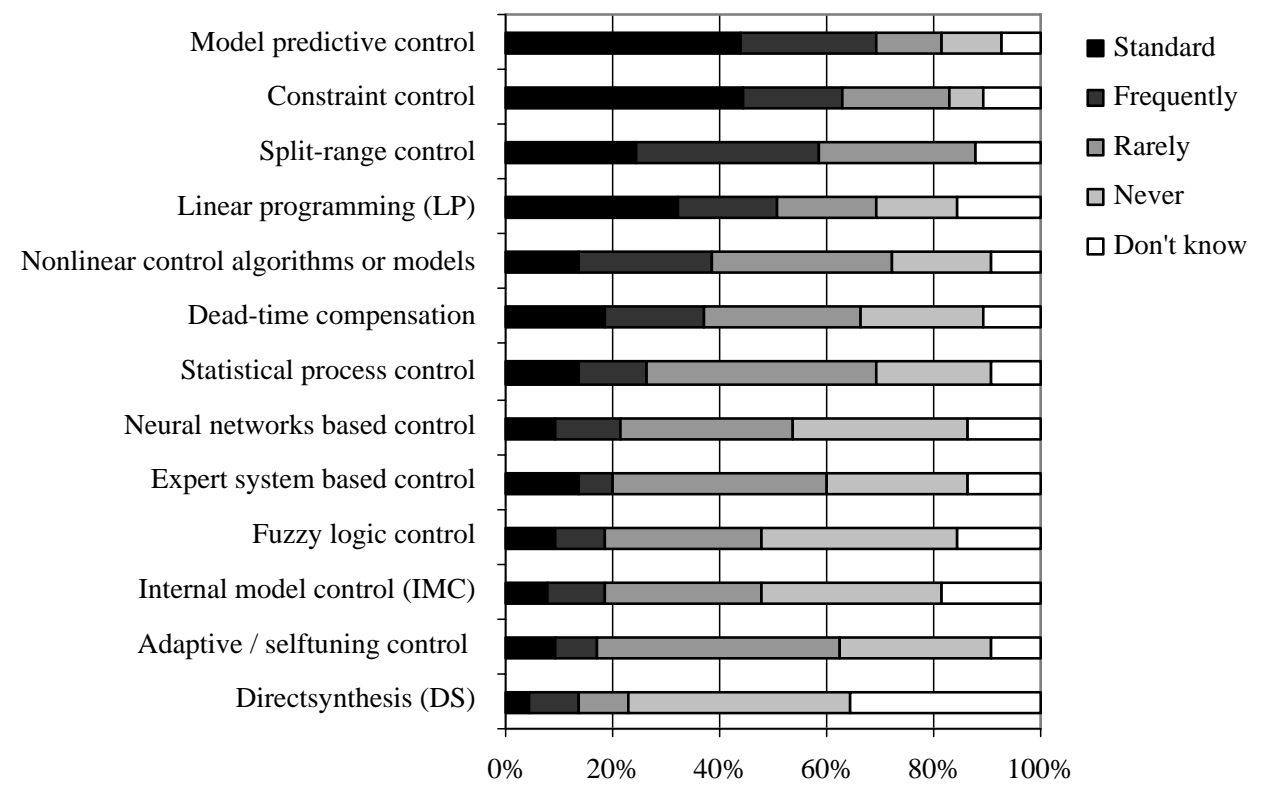

Figure 3.4: Industrial use of advanced process control methods (Bauer and Craig, 2008) 


\subsection{Step 3: Estimation of performance and initial eco- nomic assessment}

\subsubsection{Estimate the standard deviation improvement $\left(\sigma_{e s t i}-\sigma_{b a s e}\right)$ due to the advanced controller}

After the advanced controller has been designed, its functional performance needs to be estimated and compared to the base case. Functional performance is usually expressed in terms of a variance $\left(\sigma_{\text {esti }}^{2}\right)$ reduction of the CVs. As far as variation is concerned, authors with an industrial background prefer using the standard deviation, which gives the average size of deviation from the operating point (Tolfo, 1983; Latour et al., 1986). However, authors of academic papers tend to use variance (Muske, 2003; Zhou and Forbes, 2003).

\subsubsection{1 $\sigma_{e s t i}$ estimate using a fixed percentage reduction}

A reduction in standard deviation is often assumed to be a fixed percentage in many reported industrial applications. The standard deviation reduction adopted most commonly is $50 \%$ of the base case (Martin, 2004; Latour, 1992; Martin et al., 1991; Latour, 1976). Even larger reductions such as $50-66 \%$ (Stout and Cline, 1976), 85\% (Stout, 1960) or even up to 90\% (Tolfo, 1983; Latour et al., 1986) are also assumed. A lower value of only $35 \%$ is proposed in Bozenhardt and Dybeck (1986). Note that the reduction of variance is a result of control improvement instead of comparing the no-control with the controlled case (Bauer and Craig, 2008). A fixed percentage reduction in standard deviation needs to be used with caution, even though it is still being used often (Muske, 2003; Martin, 2004), as many estimates were made more than 20 years ago and advances have been made in the theory and applications of control systems and in the measurement instrumentation and final control elements.

\subsubsection{2 $\sigma_{\text {esti }}$ estimate using a simulation study}

The estimate of the variance can also be obtained using a simulation study based on the newly-designed controller and the process model (Friedmann, 2006; Zhou and Forbes, 2003). Process disturbances, however, need to be considered sufficiently to make the estimate as close as possible to the value that will be obtained after the control upgrade.

\subsubsection{Determine the optimal operating point $\left(\mu_{e s t i}\right)$ for the advanced controller}

Similar to the estimation conducted in step 1.6, based on $\sigma_{\text {esti }}$ estimated in step 1.9, the optimal operating point for the advanced controller $\mu_{\text {esti }}$ can be estimated. When the optimisation-based method is used, the PFs also need to be used. The operating points are the actual average values of $\mathrm{CVs}$ instead of setpoints. Note that although the operating point obtained will be used as setpoints for CVs (i.e. the values of setpoints are the same as the operating point), 
when the system disturbances are large and controllable, the setpoints may not be realised. $\mu_{e s t i}$ and $\sigma_{e s t i}$ are used to determine the PDFs of CVs, as described in step 1.2.

\subsubsection{Estimate the economic performance index with the advanced controller $\left(\Psi_{\text {esti }}\right)$}

In order to determine whether it is worth implementing the newly designed controller, it is necessary to estimate its economic performance to see whether it could yield significant profits. $\Psi_{\text {esti }}$ is estimated using the PFs developed in step 1.3 and PDFs estimated in step 1.10, using the continuous derivation method, as conducted in steps 1.4 and 1.7.

\subsubsection{Do fixed cost estimation}

PFs contain information on how the process cost/benefit will vary with the change of CVs. The benefit tends to depend on how well the controller works, compared to cost. Generally, the cost of a process involves instrumentation, control system hardware and software, application software, installation and commissioning, training, fixed operating cost and variable operating cost. However, in all the categories of cost, only the variable operating cost usually changes with controller performance. Other costs need to be considered in the stage of economic project evaluation, which is elaborated on in Friedmann (2006).

Cost estimation should begin with some idea of how much expenditure a project can support. An estimate of benefits and organisational guidelines will provide a rough limit on acceptable costs. The next step in cost estimation is identification of the categories that must be estimated. These will depend on the nature of the project. Modernisation of controls for an old unit will include replacement of sensors and transmitters, the purchase and configuration of a distributed control system, installation, and training of operators and maintenance personnel. It may also include the addition of new sensors, engineering of new control schemes and software to link the system with a plant-wide network. A smaller project may require only the addition of a few measurements, reconfiguration or reprogramming of one unit on an existing distributed control system and configuration of one or two displays.

\subsubsection{Conduct initial economic project evaluation and decision mak- ing}

\subsubsection{Derive performance improvement indices}

From $\Psi_{\text {base }}$ and $\Psi_{m v}$ obtained in steps 1.4 and 1.7 respectively, the estimated performance improvement of a minimum variance controller compared to the base case is

$$
\Delta \Psi_{m v}=\Psi_{m v}-\Psi_{\text {base }}
$$

In addition, from $\Psi_{\text {esti }}$ obtained in step 1.11, the estimated performance improvement of an 
advanced controller compared to the base case is therefore

$$
\Delta \Psi_{e s t i}=\Psi_{e s t i}-\Psi_{\text {base }}
$$

Then, the percentage value of the performance improvement due to the advanced controller compared to that resulting from the minimum variance controller is

$$
\lambda=\frac{\Delta \Psi_{e s t i}}{\Delta \Psi_{m v}}
$$

$\lambda$ offers useful information of a fixed percentage reduction of $\sigma_{\text {esti }}$ (Bauer et al., 2007). If $\lambda$ is bigger than one, it means the $\sigma_{\text {esti }}$ reduction is over-estimated and the estimated performance improvement will not be realisable. If $\lambda$ is less than but close to one and $\Delta \Psi_{\text {esti }}$ is adequately large, then the new controller tends to be implemented.

It is important to note that when the minimum variance is big and the potential improvement in economic performance is small, re-engineering rather than improved control might be a suitable option.

\subsubsection{Economic project evaluation}

In economic terms, a project involves a series of cash flows over time. Firstly, cash flows out as costs are incurred at the start and during the project. Cash inflows usually commence once the project is complete. Net cash flow is defined as the difference between cash inflows and cash outflows over a given period (Friedmann, 2006). Costs include initial cost (initial cost of a capital project) and operating costs (recurring costs incurred while running a facility), as described in step 1.12. Operating costs are classified into fixed costs (unaffected by production rate) and variable costs (vary with production rate). A cash flow table gives profiles indicating how the cash flows in and out at every given period, often a year, and cash flows $(C F)$ are benefits minus operating costs. The four most frequently used methods for economic project evaluation are payback period, return on investment, net present value and internal rate of return (Friedmann, 2006). The first two are non-discounted evaluation methods and the latter two are discounted methods.

Payback period $(P P)$ is the time required to recover the project first cost $(F C)$ (Ross et al., 1992), which can be obtained as follows

$$
F C=\int_{0}^{P P} C F d t
$$

Return on investment $(R O I)$ is the percentage value of the ratio of average yearly operating cash flow to initial cost, shown as follows

$$
R O I=100 \times\left[\sum_{1}^{n}\left(C F_{i}\right) / n\right] /(F C)
$$

where $n$ refers to the year number associated with the project, which holds in the sequel of this subsection. PP and ROI have similar features. Both of them are easy to understand and easy 
to obtain, but neither accounts for the time value of money. However for both the net present value $(N P V)$ and internal rate of return $(I R R)$ the time value is considered by discounting future cash flows as a function of time (Allen, 1991). The time value of money accounts for the influence of interest $(k)$ or the cost of capital. The present value $(P V)$ of a future value $(F V)$ in $n$ years is given by

$$
P V=F V_{n} /(1+k)^{n}
$$

The NPV of a series of cash flows over $n$ years can be derived by repeating the calculation of Eq. (3.21), being

$$
N P V=C F_{0}+\sum_{i=1}^{n}\left(C F_{i} /(1+k)^{i}\right)
$$

The project will more likely be approved if $N P V$ is positive, as a positive $N P V$ indicates that investment in the project will earn at a rate of return bigger than $k$. The $I R R$ is the value of $r$ that will result in a zero $N P V$. The $I R R$ is the most important alternative of $N P V$ (Ross et al., 1992). The advantage of discounted methods over non-discounted ones lies in their consistent valuation of future cash flows.

It is straightforward to convert $\Delta \Psi_{\text {esti }}$ to the annual profits resulting from an advanced controller compared to the base case. Subsequently, with information such as the initial cost, the cash flow table can be established and economic project evaluation can be conducted using one of the methods discussed above. If the evaluation result is satisfactory, the advanced controller will be implemented. Otherwise, the advanced controller needs to be re-designed and the process will return to step 1.8 .

\subsection{Step 4: Implementation of the advanced controller}

If the results of the initial economic project evaluation conform to the requirement of the management, implementation of the advanced controller will be approved. It usually takes considerable resources and time to implement a new control system.

\subsection{Step 5: Calculation of performance and success as- sessment}

\subsubsection{Collect data of controlled variables of interest}

After implementation of the advanced controller, it should be run at the optimum operating point determined in step 1.10. for a sufficiently long time such that the controller is exposed to most possible disturbance scenarios. The time series of CVs are usually stored in a database after being retrieved at each sampling time. The real plant data are then available. Requirements for collecting data are the same as those needed in step 1.1. 


\subsubsection{Determine the probability density functions of controlled vari- ables}

Obtain $\mu$ and $\sigma$ of the time series of CVs and then derive the PDFs of the CVs. When the economic performances of the CVs are considered independently, individual PDFs are obtained. However, when they are examined together, JPDFs need to be obtained.

\subsubsection{Calculate the economic performance index of the advanced controller $\left(\Psi_{a d v}\right)$}

Unlike the EPI estimation for the advanced controller conducted in step 1.11, the EPI of the advanced controller $\left(\Psi_{a d v}\right)$ can now be calculated based on the real plant data using the PDFs obtained in step 2.3 and PFs. Furthermore, since the time series are available, the discrete calculation method can also be used alternatively for the calculation.

\subsubsection{Calculate the achieved profit $\left(\Psi_{a d v}-\Psi_{b a s e}\right)$}

The performance improvement of the advanced controller compared to that for the base case is

$$
\Delta \Psi_{a d v}=\Psi_{a d v}-\Psi_{b a s e}
$$

\subsubsection{Derive the new optimal operating point $\left(\mu_{a d v 2}\right)$ based on the standard deviation obtained in Step $2.3\left(\sigma_{a d v}\right)$}

It is quite possible for the $\sigma$ of CVs of the advanced controller to differ from the value estimated in step 1.9, and then it is necessary to re-derive the optimal operating point based on the current $\sigma$ and run the controller at this new operating point. The derivation method is the same as that used in step 1.6. The newly obtained operating point will help provide better economic performance.

\subsubsection{Update the minimum variance $\left(\sigma_{m v 2}^{2}\right)$ estimate and maximum achievable performance $\left(\Psi_{m v 2}\right)$}

The minimum variance can also be updated based on real plant data, as done in step 1.5. The operating point that corresponds to the newly estimated minimum variance can then be obtained, as done in step 1.6. Afterwards, the new maximum achievable performance $\left(\Psi_{m v 2}\right)$ is derived, as done in step 1.7 . 


\subsubsection{Do statistical experiments to examine economic improvement}

\subsubsection{Motivation and general considerations of statistical experiments}

The most common way to investigate the economic improvement of the advanced controller is to switch between the old and new controller to control the process (Bauer and Craig, 2008) and detect the improvement. There are two types of experiments that are used commonly but tend to give misleading results, i.e. a 'month-on, month-off' experiment and 'comparing performance of adjacent but independent circuits' (Craig and Koch, 2003). With these two types of experiments, the difference in the economic performance of the controllers can easily be overwhelmed by various other factors such as the differences in the circuits themselves, disturbances, instrumentation, etc. In order to distribute the influence of various factors and allow fair comparison, statistical experiments need to be used (Napier-Munn, 1995). The preferred way is to use both the old and new controller to control the same process in a random way based on a carefully designed experiment. Comparative experimental techniques can be classified approximately in terms of replication, blocking and randomisation (Chatfield, 1983). Most comparative experimental techniques take either one of these divisions or a combination of two or three.

\subsubsection{Experimental steps}

A statistical experiment can be done with the following steps:

1. Modelling of disturbances (applicable for simulation studies, but not for real plant tests)

2. Stating the hypothesis (regarding the $\mu$ and $\sigma$ for both controllers)

3. Experimental design (random sequence generation, experiment duration)

4. Data generation and hypothesis testing ( $F$-test and $t$-test are often used to examine the variance difference and average difference respectively).

\subsubsection{Derive the economic performance improvement of the new controller}

When the experiment is done, the time series of all the CVs of each run for both the old and advanced controllers are available. The old controller is used $N_{\text {old }}$ times and the new controller $N_{\text {new }}$ times. For the old controller, for the time series of a CV $y_{j}$ obtained in $i^{\text {th }}$ run, the PDF $\left(f_{\text {old } i}\left(y_{j}\right)\right)$ is determined from the average and standard deviations that are easily obtained, and then the EPI is

$$
\Psi_{y_{j}}^{\text {old } \_i}=\int_{-\infty}^{\infty} v^{\text {old_i }}\left(y_{j}\right) f\left(y_{j}\right) d y_{j}
$$

When the univariate analysis is used, the overall EPI for the $i^{\text {th }}$ run of the old controller is therefore

$$
\Psi^{\text {old_ } i}=\sum_{j=1}^{p} \Psi_{y_{j}}^{o l d \_i}
$$


The average EPI of the old controller is therefore

$$
\Psi^{\text {old }}=\frac{1}{N_{\text {old }}} \sum_{i=1}^{N_{\text {old }}} \Psi^{\text {old } i}
$$

The average EPI of the new controller $\left(\Psi^{n e w}\right)$ is obtained using a similar process. The average improvement of economic performance resulting from the statistical experiment is

$$
\Delta \Psi_{\text {sta }}=\Psi^{\text {new }}-\Psi^{\text {old }}
$$

\subsubsection{Conduct final economic project evaluation}

Compare the $\Psi_{e s t i}$ and $\Psi_{a d v}$ to see how great a percentage of the estimated performance improvement has been realised. In addition, according to the $\Psi_{a d v}$, a new economic project evaluation is done, as conducted in step 1.12, to have a new idea of how soon the initial cost will be recovered and how the profits will change over time.

Compare the $\Psi_{a d v}$ and $\Psi_{m v 2}$ to see the difference and the possibility of improving the performance further by re-tuning the advanced controller. Compare $\Psi_{m v}$ and $\Psi_{m v 2}$ to see how accurate the maximum achievable performance estimation done before the control upgrade is.

By comparing the $\Delta \Psi_{\text {sta }}$ and $\Delta \Psi_{a d v}$, it can be seen whether the accuracy of a simple comparison is satisfactory. The economic performance needs to re-evaluated periodically even after the implementation of the advanced controller.

\subsection{Conclusion}

In this chapter, the EPA framework proposed in Bauer and Craig (2008) is reviewed critically in order to facilitate the EPA of the ROM milling circuit that will be done in Chapter 7 and to reveal more information of EPA methods. 


\section{Chapter 4}

\section{Performance functions and a multivariate economic assessment of a furnace}

\subsection{Introduction}

Performance functions play a crucial role in advanced methods of economic assessment of control systems (Bauer and Craig, 2008). In order to develop a methodology of developing PFs, several case studies need to be conducted. A batch process, an EAF, is studied in this chapter. Whether the IPFs/JPFs will be developed depends on whether the multivariate nature of the process's economic model is considered. IPFs are used by default. When different process variables are assumed to be independent, the IPF of each crucial process variable will be developed. When the multivariate nature of the process is considered, the JPF of process variables will be considered. In this chapter, firstly the development of IPFs for the EAF, as conducted in Oosthuizen et al. (2004), is investigated and important ideas used for the development are abstracted. Then the focus is put on the multivariate nature of the process and the JPFs of the EAF are developed based on the IPFs developed. The JPFs are used subsequently for a multivariate EPA for the EAF to show how the JPFs will influence the results of the economic assessment. The content of this chapter is also reported in Wei et al. (2007).

The organisation of this chapter is described below. Section 4.2 provides the background of the EAF discussed and a short description of the simulation and economic performance index. The development of IPFs of the EAF, as conducted in Oosthuizen et al. (2004), is reviewed and important ideas used are abstracted in Section 4.3. Then in Section 4.4 the joint performance functions of the EAF are developed. The methodology of the assessment and relevant steps required to conduct the assessment are given in Section 4.5. In Section 4.6, the methodology is applied and the results are given. The conclusion follows in Section 4.7. 


\subsection{Process background and economic model}

\subsubsection{Description of the electric arc furnace}

EAFs are commonly used in the steel industry to convert iron into steel with three-phase electric power as the main energy source (Bekker et al., 2000); see Fig. 4.1. Graphite electrodes provide the three-phase arc. The objective of EAF steel-making is to increase the steel temperature to facilitate further processing and to remove impurities from the molten steel (Lankford et al., 1985).

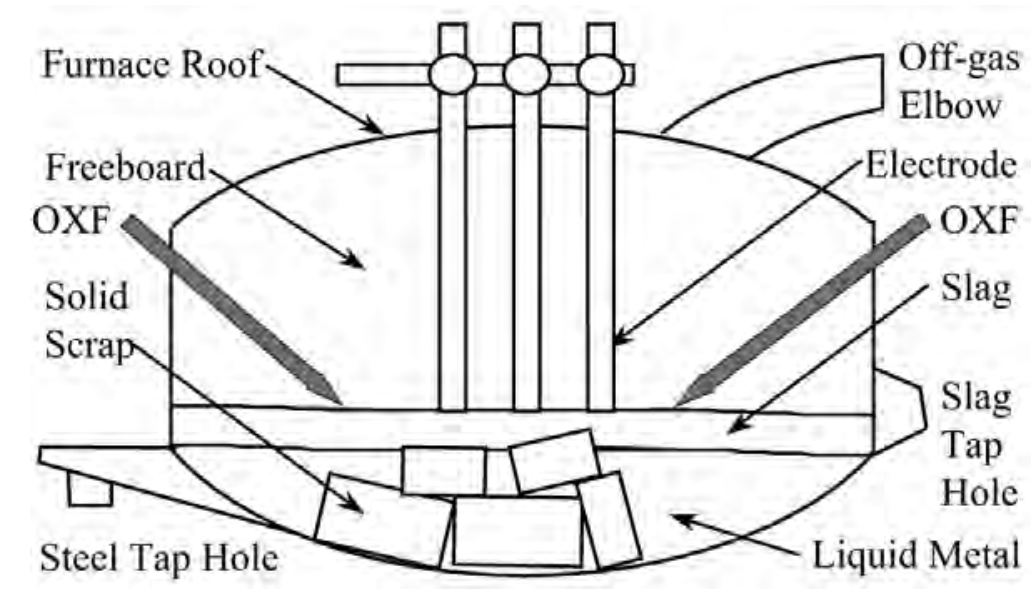

Figure 4.1: The structure of an EAF

An EAF is a batch process in which the process steps are as follows (Lankford et al., 1985): The melting period starts after the charging of scrap metal. The electrodes bore through the metallic charge, producing a pool of molten metal in the hearth. In the EAF considered, hot metal is also charged after this primary melting process. After the melting period follows the oxidising and temperature-adjusting process during which the impurity level in the melt is decreased and the temperature is raised to the level required for tapping. For the process considered, direct reduced iron (DRI) is charged continuously into the furnace. Oxygen is also blown continuously into the furnace, acting as an additional energy source.

A slag layer is formed during the melting process. The layer floats on the steel melt and acts as a blanket to the molten metal, as well as a reservoir for impurities. In order to facilitate the elimination of impurities, fluxes are added to the slag to modify its composition. The slag layer furthermore protects the furnace walls from the arc and ensures maximum energy transfer to the steel melt. The slag is often foamed by injecting graphite into the slag layer. Foaming also occurs naturally owing to gas bubbling through the slag layer.

Gases produced during steel-making are removed via the off-gas system (not shown in Fig. 4.1). The off-gas system consists of an off-gas fan, a slip-gap, a water-cooled duct and a bag-house. The off-gas fan removes the gases from the EAF. The slip-gap regulates the ratio of gases extracted from the EAF relative to the air entrained from the atmosphere. The bag-house acts as a filter removing dust particles from the off-gas before emission into the atmosphere. 


\subsubsection{Economic performance index of the electric arc furnace}

The EPI of the EAF is defined as the additional production cost compared to the typical operational condition described in Bekker et al. (1999). The production cost of the EAF can be separated into two types: the cost of 13 feed components $\left(C_{f}\right)$ and the cost $\left(C_{c v}\right)$ that results from the CVs violating their specifications (Oosthuizen et al., 2004). The overall additional performance is the sum of $\Delta C_{f}$ and $\Delta C_{c v}$ :

$$
\Delta C=\Delta C_{f}+\Delta C_{c v}
$$

The 13 feed components are scrap, electric power, maintenance, hot metal, direct reduced iron, electrodes, refractories, flux, labour, investment, oxygen, off-gas power and graphite. The additional cost of the feed components $\Delta C_{f}$ is relatively easy to quantify and therefore not the focus of this investigation. The cost that results from the CVs violating their constraints $\left(\Delta C_{c v}\right)$ is related to performance functions of the CVs.

\subsubsection{Process variables and control strategies}

The CVs, manipulated variables (MVs) and disturbances of the EAF model are listed in Fig. 4.2. The simulation model, model predictive control (MPC) algorithm, parameters and experiment design used in this work conform to those in Oosthuizen et al. (2004), which demonstrates that an MPC-controlled EAF may be not only a technical success, but an economic one as well.

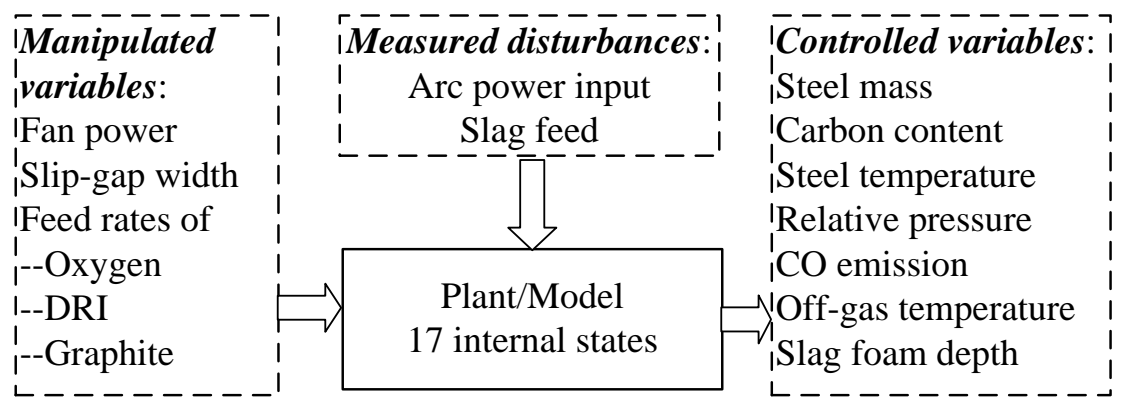

Figure 4.2: The EAF model variables (Oosthuizen et al., 2004)

As far as control strategies are concerned, they are considered to reach two objectives: functional performance and economic performance. For functional performance, the control is intended to satisfy the CV setpoint tracking despite various disturbances. For economic performance, the control is expected to minimise the EPI.

\subsection{Review of the development of individual performance functions of the furnace}

The setpoints of controlled variables are determined based on various considerations. When the controlled variables violate the setpoints, there will be some direct or indirect economic impact. The development of IPFs of the CVs of the EAF is reviewed in this subsection and the IPFs are given in Fig. 4.3. 

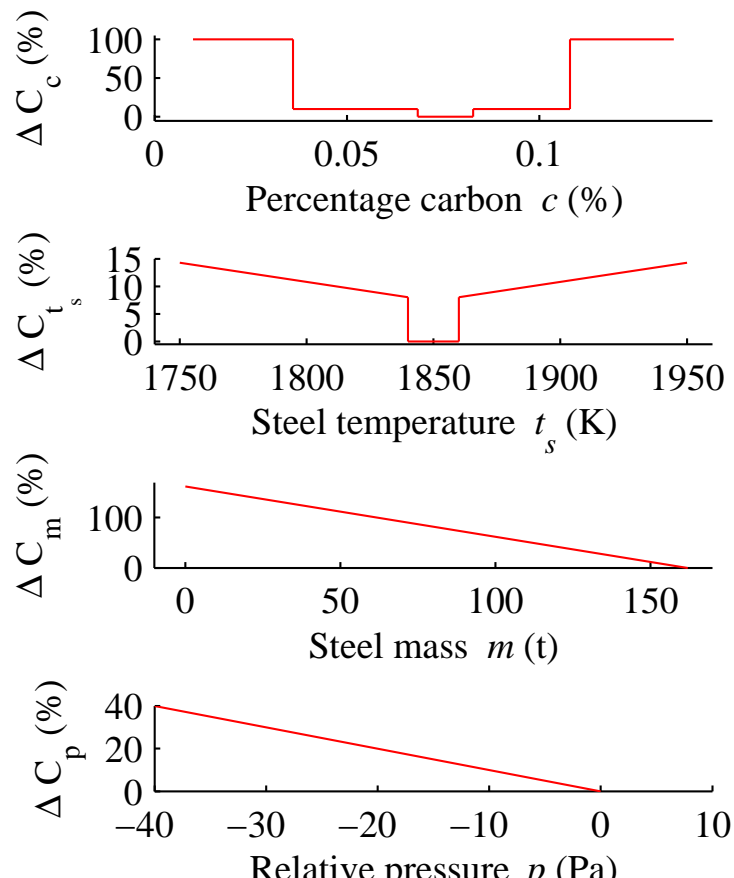

$$
\begin{gathered}
\Delta C_{c}= \begin{cases}100 \%, \quad c<0.036 \%, \\
10 \%, \quad 0.036 \% \leq c<0.0684 \% \\
0 \%, \quad 0.0684 \% \leq c<0.0828 \% \\
10 \%, \quad 0.0828 \% \leq c<0.108 \% \\
100 \%, \quad c \geq 0.108 \%\end{cases} \\
\Delta C_{t_{s}}= \begin{cases}8 \%-\frac{0.063 \%}{\mathrm{~K}}\left(t_{s}-1840 \mathrm{~K}\right), t_{s}<1840 \mathrm{~K} \\
0 \%, \quad 1840 \mathrm{~K} \leq t_{s}<1860 \mathrm{~K} \\
8 \%+\frac{0.063 \%}{\mathrm{~K}}\left(t_{s}-1860 \mathrm{~K}\right), t_{s} \geq 1860 \mathrm{~K}\end{cases} \\
\Delta C_{m}= \begin{cases}0 \%, & m \geq 162.1 \mathrm{t} \\
\frac{1 \%}{\mathrm{t}}(162.1 \mathrm{t}-m), & m<162.1 \mathrm{t}\end{cases} \\
\Delta C_{p}= \begin{cases}\frac{-0.1255 \%}{\mathrm{~Pa}}(p), & p<0 \mathrm{~Pa} \\
0 \%, & p \geq 0 \mathrm{~Pa}\end{cases} \\
\Delta C_{t_{o g}}= \begin{cases}0 \%, & t_{o g}<73 \mathrm{~K} \\
3 \%, & t_{o g} \geq 773 \mathrm{~K}\end{cases} \\
\begin{array}{l}
0 \%, \quad 30 \mathrm{~cm} \leq d<90 \mathrm{~cm} \\
0 \%(\mathrm{~cm}
\end{array}
\end{gathered}
$$

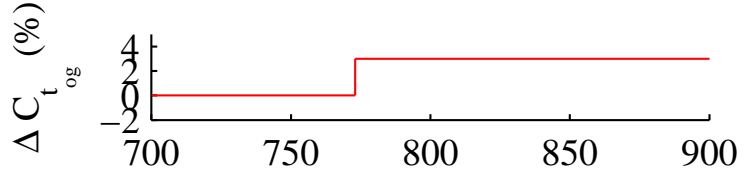

Off-gas temperature $t_{o g}(\mathrm{~K})$

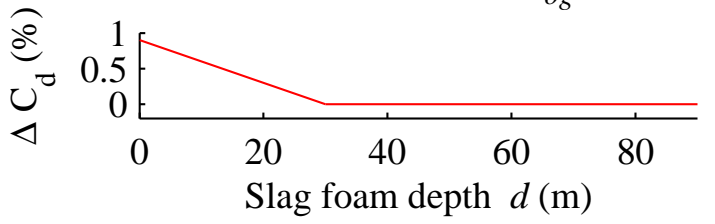

Figure 4.3: IPFs of the EAF

\subsubsection{Percentage carbon}

The carbon content of the molten steel has very little effect on the cost at any time during the tap. However, when the steel is tapped, the carbon content should satisfy some specifications. The allowable deviations on carbon content vary for different types of steel produced, but usually have a range of approximately 20\%. If the carbon content is completely off target, the melt might be scrapped, which means the melt cost would be more than double. If the specification on carbon content is not fulfilled, some action needs to be taken, otherwise the melt will be scrapped. The action comprises switching on the arc for an additional period, blowing more oxygen, or adding some materials to the melt. The cost of these actions is usually small compared to the cost of the melt. Blowing oxygen for another 5 minutes would have a small impact on the total cost of a tap. Oxygen consumption contributes around $0.7 \%$ to the total operating cost, and blowing oxygen for an additional 5 minutes would increase the total operating cost by less than $\mathbf{0 . 1 \%}$.

Another matter of concern is that there is a delay of almost 5 minutes between taking a steel sample and getting the results. If it takes 5 minutes to have corrective action taken (Schutte, 2000), 10 minutes would pass before the final composition is known and tapping can commence. 
In many circumstances the EAF processing is a bottleneck, which means a downstream process is persistently waiting for steel from the EAF, i.e. production would have to slow down if the steel from the EAF is delayed.

If it is assumed that the typical processing time for a tap is 100 minutes (Bekker, 1999), the preceding reasoning indicates that a production loss of $10 \%$ would be suffered owing to a too big deviation in carbon content. Although there are some other losses (Schutte, 2000), the cost owing to production losses accounts for the biggest part of the cost due to variations in carbon content (Schutte, 2000). The relevant PF is referred to in Fig. 4.3.

Comment: The production loss due to a process delay is the focus.

\subsubsection{Steel temperature}

The steel temperature is supposed to be high enough to ensure smooth tapping of the melt and also to have sufficient heat as required by the downstream metallurgical processes. A too high temperature may result in detrimental reactions with the melt, more inclusions in the steel and increasing EAF lining wear. Considering none of the impacts resulting from a too high temperature is modelled, the cost will be considered for a too low temperature and it is assumed that a symmetrical cost distribution exists.

The tapping temperature is allowed to vary by approximately $+/-10 \mathrm{~K}$ (Schutte, 2000). The throughput is also assumed to be a determining factor in the additional cost due to a too low temperature. When the temperature is too low, electrical energy (arc) or chemical energy (oxygen) will be added to increase the temperature. The cost of the additions is once again small compared to the cost of lost production, but may become considerable for large temperature differences. An average temperature measurement delay of 4 minutes is used (Schutte, 2000), implying a $4 \%$ increase in tapping time.

The heat loss during the measurement delay needs to be considered too. The heat loss can be calculated according to the principle of the temperature rate of change in the EAF as described in Bekker (1999). When corrective action is needed, the steel would be heated not only by the temperature difference as measured, but also the temperature losses that happen during the measurement delay. Considering this fact and the restrictions on the maximum power input to the EAF, the tapping time would be increased by not only the $4 \%$ of measurement delay, but also another $4 \%$ in recovering energy losses during the measurement delay. It is assumed that the tapping temperature is never far below the required temperature, which might cause the melt to solidify inside the EAF. The cost of a frozen melt is not investigated. The PF for steel temperature can be seen in Fig. 4.3.

Comment: The process delay is also a focus.

\subsubsection{Off-gas temperature}

The off-gas temperature needs to be restrained to prevent the bag-house from exploding. If danger is detected, a trip switch will ensure that the EAF processing is halted. The time 
between the EAF being switched off and on again relates to a production loss, similar to the cases for carbon content and steel temperature. It is assumed in this analysis that 3 minutes of production time is wasted for each time the off-gas temperature exceeds a limit of $773 \mathrm{~K}$ (Schutte, 2000). The cooling losses during this period are approximately $0.2 \%$ of the whole cost of an EAF tap, which is once again negligible in contrast to the cost of lost production. Exceeding the off-gas temperature limit would then cause an increase in production cost of 3\%, considering that the duration of a typical tap is around 100 minutes.

The measurement of the off-gas temperature is done in real time, so there is no additional delay with such a shutdown. There is neither an advantage nor a disadvantage when the off-gas temperature is low.

Comment: The process delay is also the focus here.

\subsubsection{Slag foam depth}

Foamy slag has several advantages, including decreased heat loss to the side-walls, improved heat transfer from the arc to the steel, reduced power and voltage variations, reduced noise and reduced electrode and furnace lining consumption. A slag foam depth much higher than the arc length has neither advantage nor disadvantage, except for increased feed consumption in reaching the depth. It might, however, have some operational disadvantage, since a very big slag volume will have to be tapped. A slag foam depth too low to cover the arc has a negative economic impact, as it will lead to inefficient power transfer to the bath and increased heat losses.

When the depth is zero, the additional cost is considered as $0.9 \%$, which is derived from the waste of electrical energy. The wastage is calculated based on the relation described in Bekker (1999) and Jiang and Fruehan (1991). When the depth is more than $30 \mathrm{~cm}$, there is no additional cost. The relation between the additional cost and the depth is assumed to be linear. The PF can be seen in Fig. 4.3.

\subsubsection{Steel mass}

If the EAF is assumed to be the bottleneck, any tap not using the maximum capacity would translate to a production loss. Exceeding the capacity of the EAF may lead to some problems that are, however, not considered in this analysis. For the purpose of this study, it is assumed that increased capacity has neither an advantage nor a disadvantage. The overall cost with a too high steel mass will, however, be higher because of the increased feed and energy consumption, but this increase in cost will correspond to the increase in the value of the tap. In such circumstances it is regarded that the increased cost could be offset by the increased benefit of the tap. This is not the case for other CVs, since otherwise there is no additional benefit anyway. The PF of the steel mass is shown in Fig. 4.3. 


\subsubsection{CO emission}

The off-gas system of an EAF is devised to ensure that CO is combusted sufficiently with a large safety margin. Whereas excessive $\mathrm{CO}$ emissions may cause a plant shutdown until effective CO emission control measures have been taken, the cost of excessive CO emission can be large. In this analysis the cost of a forced plant shutdown is estimated as the cost of 10 taps. It may be underestimated, but the cost is partially counteracted by the fact that $\mathrm{CO}$ emissions are usually averaged over a given period, and one peak $\mathrm{CO}$ emission of short duration would thus not cause legislative action against the plant. The chance is small that a plant would be forced to shut down as a result of too high CO emissions.

\subsubsection{Relative furnace pressure}

Ideally the relative furnace pressure should be controlled at $0 \mathrm{~Pa}$, which is actually not practical, as any increase in relative pressure would result in the emission of dust and fumes into the workshop, leading to a safety hazard. A too low relative pressure is also not viable, as energy will be wasted owing to unnecessary extraction of hot gases. The pressure is usually controlled at $-5 \mathrm{~Pa}$ or lower, because this is regarded as a good balance between efficiency and safety.

It can be seen that the additional energy extracted with the hot gases is approximately 337 $\mathrm{kW} / \mathrm{Pa}$ by substituting typical values into the EAF model described in Bekker (1999). Controlling the relative pressure at $-5 \mathrm{~Pa}$ rather than $0 \mathrm{~Pa}$ would lead to additional energy usage of nearly 1.7 MW, accounting for $4 \%$ of the overall electrical input. Considering that electrical energy constitutes approximately $14.9 \%$ of the total EAF operational cost, the PF of the relative pressure can be obtained, as shown in Fig. 4.3 .

The analysis for positive relative pressure is much more complicated, as the influence on the health of workers is difficult to quantify. This analysis focuses only on negative relative pressure. It is assumed that a positive pressure of short duration will not have a significant influence on the health of workers and the negative pressure setpoint would be selected low enough to prevent negative relative pressure. Improved regulation may help to set up a higher relative pressure setpoint without compromising the health and safety of the workers.

\subsection{Development of joint performance functions of the furnace}

It is assumed in Section 4.3 that CVs are statistically independent, which is not always the case. If two CVs violate their specifications simultaneously, the additional cost could overlap or even be the same, and not be just the sum of two independent costs. Not considering the dependence of CVs can therefore lead to the incorrect estimation of $\Delta C_{c v}$, as will be shown later. In this section the JPFs of the EAF considered are developed. Firstly the dependence of CVs is investigated and then JPFs are developed for the dependent CVs. 


\subsubsection{Determining the dependence of controlled variables}

The economic performance indices associated with different CVs are often summed to give the overall economic performance index. The basic assumption when summing benefits is that the CVs are independent. This is not always the case. Considering the multivariate nature of an economic model may lead to a more accurate estimate of EPI. To this end, the dependence of CVs needs to be examined first. A powerful graph for showing the relationship between two variables is the scatter plot. Here, for each batch of the EAF operation, a point is plotted in a two-dimensional plane where the $\mathrm{x}$-axis represents a first variable and the $\mathrm{y}$-axis a second variable. The scatter plot can be interpreted by searching for an overall pattern by visual inspection. The pattern also reveals the direction, form and strength of the dependence of two variables (Moore and McCabe, 1989). Two variables are associated positively when aboveaverage values of one variable tend to accompany above-average values of the other variable, and below-average values tend to occur together. Two variables are associated negatively when above-average values of one variable accompany below-average values of the other variable, and vice versa (Moore and McCabe, 1989). The dependence between variables for the manual and MPC controlled EAF can be determined from scatter plots of relevant CVs.

The scatter plots for the percentage carbon and off-gas temperature under manual and MPC control are shown in Fig. 4.4. Each point represents the corresponding values of the two variables for one tap, that is, 203 taps under manual control and 217 taps under MPC control. It can be seen from Fig. 4.4 that for manual control the value of the percentage carbon can be deduced from the value of the off-gas temperature, and vice versa. For instance, if the percentage carbon is known to be $0.075 \%$, the off-gas temperature will be around $835 \mathrm{~K}$. So these two variables are strongly dependent under manual control. An explanation of the dependence is that the higher the percentage carbon, the more CO is generated, hence the higher off-gas temperature.

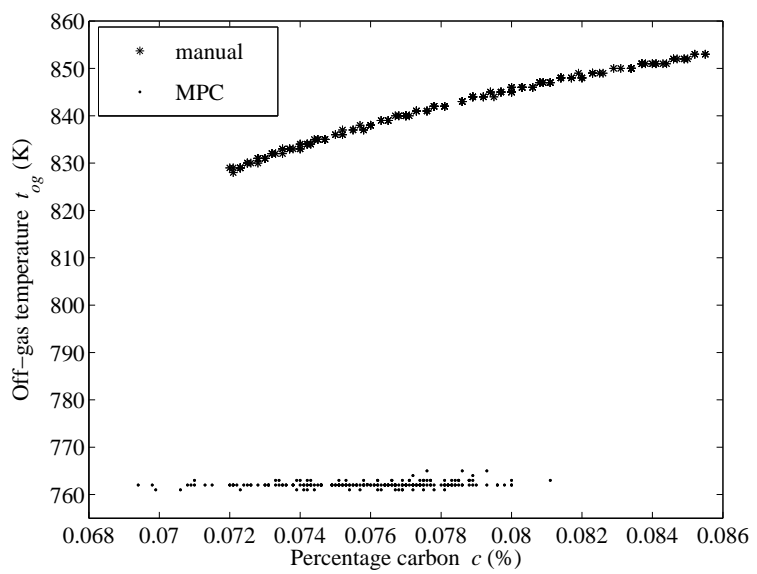

Figure 4.4: Scatter plot of percentage carbon $c$ and off-gas temperature $t_{o g}$

Under MPC control, the off-gas temperature remains almost constant and is therefore independent of the percentage carbon. This is because the constraints in the MPC controller restrict the off-gas temperature so that it remains within a relatively small range.

The scatter plots for steel temperature and steel mass under manual and MPC control are shown in Fig. 4.5. It can be seen that the steel temperature can be deduced from the steel mass, and vice versa. For example, if the steel temperature is known to be $1840 \mathrm{~K}$, the steel mass will be around $163.5 \mathrm{t}$, so these two variables are strongly dependent under manual control. The dependence of these two variables under manual control is comprehensible. For a given energy 
input, if there is more steel mass in the hearth, it is inevitable that the steel temperature will be a littler lower, according to the law of conservation of energy. However, for MPC control the steel mass cannot be determined from the steel temperature. So these two variables can be taken as independent under MPC control, again as a result of constraints imposed on CVs and MVs.

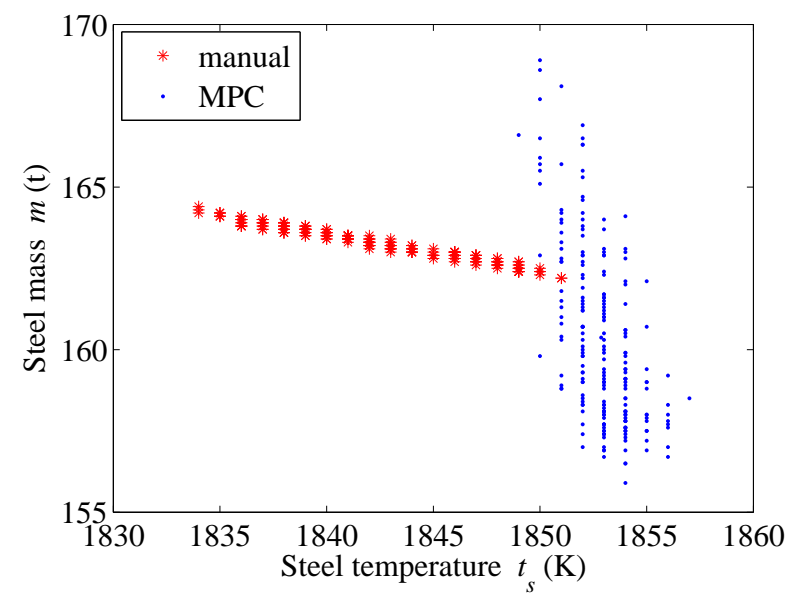

Figure 4.5: Scatter plot of steel temperature $t_{s}$ and steel mass $m$

Visual inspection of the scatter plots for all pairs of CVs indicate that there are only two pairs of variables that are dependent, namely the percentage carbon and off-gas temperature under manual control, and the steel temperature and steel mass under manual control, see Fig. 4.4 and 4.5.

\subsubsection{Developing the joint performance functions for dependent con- trolled variables}

Based on the dependence of CVs, JPFs for dependent CVs are developed. Note that when JPF is the sum of IPFs for dependent variables, the economic performance index calculation result will be the same as when the variables' dependence is neglected. In other words in these cases the development of JPF is not compulsory. When all the additional cost of dependent variables results from additional tap-to-tap time, the longest additional time will automatically compensate for the shorter additional time required. Tap-to-tap time is required to compensate for the violation of specifications. If the ways in which the additional cost is compensated for are different, the JPF is the summation of IPFs given in Section 4.2.2.

The JPF for percentage carbon and off-gas temperature is shown in Fig. 4.6. The additional cost of both variables results from an increase in tap-to-tap time when specifications are violated. If neither of them violates the specifications, the JPF is $0 \%$. If only one variable violates the specification, the joint performance function is the performance function of only that violated variable. Furthermore, if only the percentage carbon violates the specification, then the JPF is:

$$
\Delta C_{t_{o g}, c}= \begin{cases}100 \%, & c<0.036 \%, \text { and } t_{o g}<773 \mathrm{~K} \\ 10 \%, & 0.036 \% \leq c<0.0684 \%, \text { and } t_{o g}<773 \mathrm{~K} \\ 10 \%, & 0.0828 \% \leq c<0.108 \%, \text { and } t_{o g}<773 \mathrm{~K} \\ 100 \%, & c \geq 0.108 \%, \text { and } t_{o g}<773 \mathrm{~K}\end{cases}
$$




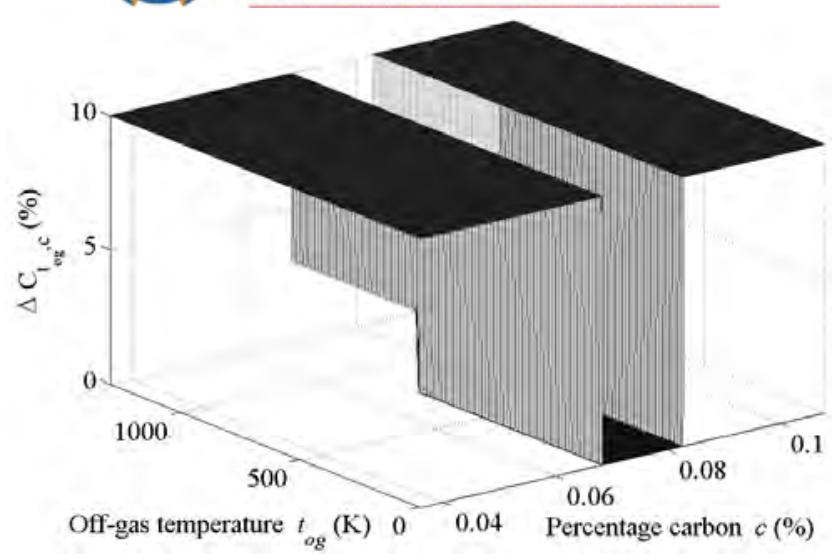

Figure 4.6: JPF of percentage carbon $c$ and off-gas temperature $t_{o g}$

If only the off-gas temperature violates the specification, then the JPF is:

$$
\Delta C_{t_{o g}, c}=3 \%, t_{o g} \geq 773 \mathrm{~K}, \text { and } 0.0684 \% \leq c<0.0828 \%
$$

If both of them violate the specifications, then there are two situations:

- If $0.036 \% \leq c<0.0684 \%$ or $0.0828 \% \leq c<0.108 \%$ then an additional ten minutes of processing is required; this is because ten more minutes are required to compensate for the violation of the percentage carbon, compared to three more minutes for the off-gas temperature. Both violations can therefore be compensated for by increasing the tap-totap time by 10 minutes. So the JPF is the maximum of the IPFs of those two variables:

$$
\Delta C_{t_{o g}, c}= \begin{cases}100 \%, & c<0.036 \%, \text { and } t_{o g} \geq 773 \mathrm{~K} \\ 10 \%, & 0.036 \% \leq c<0.0684 \%, \text { and } t_{o g} \geq 773 \mathrm{~K} \\ 10 \%, & 0.0828 \% \leq c<0.108 \%, \text { and } t_{o g} \geq 773 \mathrm{~K} \\ 100 \%, & c \geq 0.108 \%, \text { and } t_{o g} \geq 773 \mathrm{~K}\end{cases}
$$

- If $c<0.036 \%$ or $c \geq 0.108 \%$ then the percentage carbon specification is off target and the melt might have to be scrapped. This means that the melt cost would more than double. Then the JPF is:

$$
\Delta C_{t_{o g}, c}=100 \%, \quad c<0.036 \% \text { or } c \geq 0.108 \%, \text { and } t_{o g} \geq 773 \mathrm{~K}
$$

The JPF for the steel temperature and steel mass is shown in Fig. 4.7. The additional cost when violating the steel temperature specification is the increased tapping time, whereas when the steel mass specification is not met the additional cost is seen as a loss in production. Therefore, compensating for a specification violation in the steel temperature does not compensate for a violation in steel mass specification. If neither of them violates the specifications, then the $\mathrm{JPF}$ is $0 \%$. If only one of them violates the specification, then the JPF is just the IPF of that violated variable. Further, if only steel temperature violates the specification, then the JPF is:

$$
\Delta C_{t_{s}, m}= \begin{cases}8 \%-\frac{0.063 \%}{\mathrm{~K}}\left(t_{s}-1840 \mathrm{~K}\right), & t_{s}<1840 \mathrm{~K}, \text { and } m \geq 162.1 \mathrm{t} \\ 8 \%+\frac{0.063 \%}{\mathrm{~K}}\left(t_{s}-1860 \mathrm{~K}\right), & t_{s} \geq 1860 \mathrm{~K}, \text { and } m \geq 162.1 \mathrm{t}\end{cases}
$$

If only steel mass violates the specification, then the JPF is:

$$
\Delta C_{t_{s}, m}=\frac{1 \%}{\mathrm{t}}(162.1 \mathrm{t}-m), \quad m<162.1 \mathrm{t}, \text { and } 1840 \mathrm{~K} \leq t_{s}<1860 \mathrm{~K}
$$




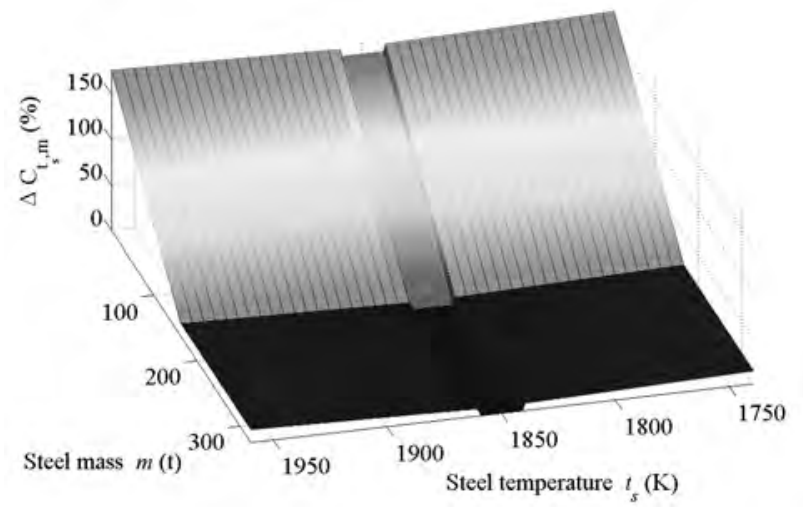

Figure 4.7: JPF of steel temperature $t_{s}$ and steel mass $m$

If both of them violate the specifications, then the JPF is the sum of IPFs of these two variables:

$$
\Delta C_{t_{s}, m}= \begin{cases}8 \%-\frac{0.063 \%}{\mathrm{~K}}\left(t_{s}-1840 \mathrm{~K}\right)+\frac{1 \%}{\mathrm{t}}(162.1 \mathrm{t}-m), & t_{s}<1840 \mathrm{~K}, \text { and } m<162.1 \mathrm{t} \\ 8 \%+\frac{0.063 \%}{\mathrm{~K}}\left(t_{s}-1860 \mathrm{~K}\right)+\frac{1 \%}{\mathrm{t}}(162.1 \mathrm{t}-m), & t_{s} \geq 1860 \mathrm{~K}, \text { and } m<162.1 \mathrm{t}\end{cases}
$$

The joint performance of the off-gas temperature and percentage carbon under manual control obtained from 203 tap simulations is shown in Fig. 4.8. The joint performance of the steel temperature and steel mass under manual control obtained from 203 tap simulations is shown in Fig. 4.9.

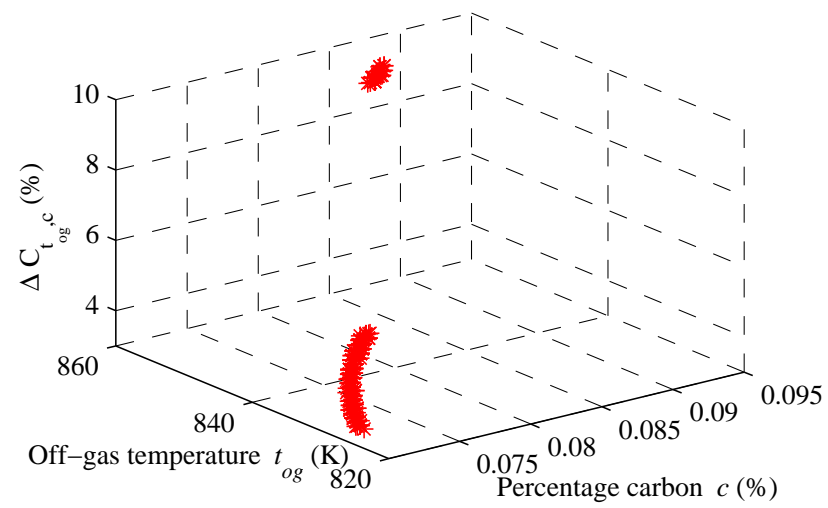

Figure 4.8: Joint performance of off-gas temperature $t_{o g}$ and percentage carbon $c$ under manual control

\subsection{Methodology for the economic assessment of the fur- nace controllers}

A randomised experiment (Craig and Koch, 2003) is conducted in a simulation environment designed to compare the economic impact of manual and MPC control as applied to an EAF model. Typical operational conditions (Bekker et al., 1999) are chosen to ensure that the experiment is as close to reality as possible. The assessment procedure is as follows: 


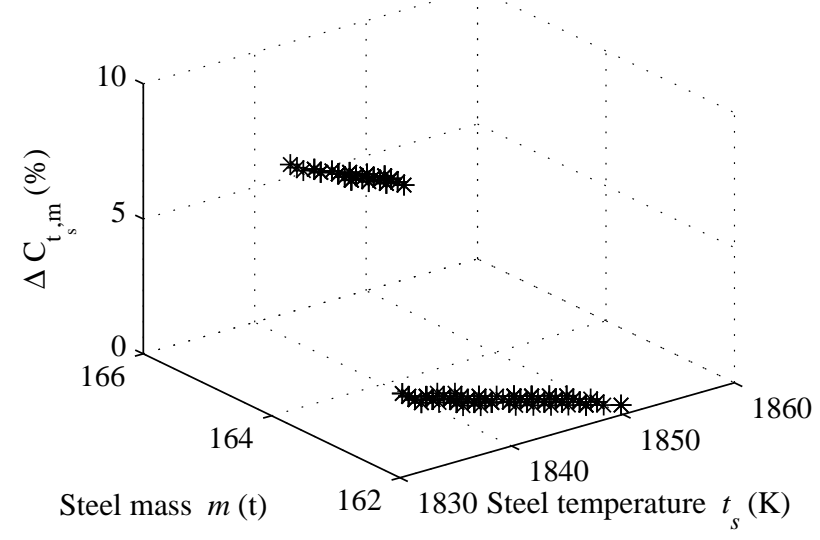

Figure 4.9: Joint performance of steel mass $m$ and steel temperature $t_{s}$ under manual control

Step 1 Conduct the experiment and obtain all the corresponding simulation data. A complete randomised designed experiment as clarified in Craig and Koch (2003) is used to compare the average economic performance of the EAF under manual and MPC control. The two controllers are used to control the EAF for different taps according to a predetermined random sequence. Each simulation is different because the power transfer rate, scrap compositions and DRI compositions are randomised to approximate typical operating conditions. The randomised experiment ensures that the generated data will be uncorrelated to the time trends of the disturbances (Oosthuizen et al., 2004). The period of the experiment covers 420 taps, 203 taps for manual control and 217 taps for MPC control.

Step 2 Determine the dependence of $C V s$. The dependence of CVs is investigated using scatter plots for all pairs of $\mathrm{CVs}$ for manual control and MPC control respectively.

Step 3 Obtain the average economic performance for the feed components. The mean and standard deviation of each feed component is derived and then the average feed cost $\left(\overline{\Delta C_{f}}\right)$ summed for manual and MPC control respectively. Then the difference in average cost of the feed components consumed is obtained. The work of this part conforms to that in Oosthuizen et al. (2004).

Step 4 Calculate the average economic performance for all independent $C V s$. If variables are independent, economic benefits are calculated from the individual performance functions as discussed in Section 4.2.2. For example, for CV relative pressure $(p)$, the average performance is obtained as follows:

$$
\overline{\Delta C}_{p}=\frac{1}{N} \sum_{j=1}^{N} \Delta C_{p}\left(p_{j}\right)
$$

where $N$ is the sample size. For manual control $N$ is 203, and for MPC control it is 217 .

Step 5 Calculate the average economic performance for all pairs of dependent CVs by developing JPFs. For dependent variables, a JPF is developed and then the joint performance of dependent variables for each tap obtained. The average value of the joint performance for the dependent variables is obtained afterwards. For example, if variables $t_{s}$ and $m$ are dependent, the average performance is obtained over all samples as follows:

$$
\overline{\Delta C}_{t_{s}, m}=\frac{1}{N} \sum_{i=1}^{N} \Delta C_{t_{s}, m}\left(t_{s i}, m_{i}\right)
$$


where $N$ is the sample size as previously defined in Eq. (7.5.2).

Step 6 Obtain the overall average performance. For both manual and MPC control, the overall average performance is calculated by summing the average performance of all independent and dependent variables, as well as the cost from the feed components:

$$
\begin{aligned}
\overline{\Delta C} & =\overline{\Delta C}_{f}+\overline{\Delta C}_{c v} \\
& =\overline{\Delta C}_{f}+\sum_{k=1}^{n_{1}} \overline{\Delta C}_{k}+\sum_{l=1}^{n_{2}} \overline{\Delta C}_{l}
\end{aligned}
$$

where $n_{1}$ is the number of independent variables and $n_{2}$ is the number of groups of dependent variables. The improvement resulting from MPC control is then derived.

Step 7 Hypothesis tests are conducted to determine whether MPC control provides a statistically significant economic improvement. A $t$-test can be used to test if there is a significant difference between two means of two populations, in this case the overall average operational cost of the EAF considered.

\subsection{Economic assessment of a model predictive controlled furnace}

In this section the methodology of assessment described in Section 4.5 is conducted to perform the economic assessment of controllers for the EAF. Simulation data from experiments are used to investigate the advantage of MPC over manual control. The overall average economic performance for manual and MPC control is determined. Then hypothesis tests are conducted to examine whether a statistically significant economic improvement occurs when using MPC control. The assessment results are given.

\subsubsection{Overall average economic performance}

The average performance of dependent $\mathrm{CVs}$ is derived as described in Eq. (4.10), based on the dependence and joint performance of the CVs. The average performance of independent variables is obtained as described in Eq. (7.5.2). Then, for both manual and MPC control, the overall average economic performance is derived as described in Eq. (4.11) of Step 6 in Section 4.5. The assessment is summarised in Table 4.1 and compared to the results obtained in Oosthuizen et al. (2004), where the dependence of CVs is not taken into account. The results from Oosthuizen et al. (2004) are modified slightly in this work and referred to as 'Assessment 2'. Although the percentage carbon and off-gas temperature are independent under MPC control, the corresponding cells are merged in the table, to emphasise the dependence of the percentage carbon and off-gas temperature under manual control. Furthermore, because the joint performance of independent variables is the sum of corresponding individual performance, the two cells can be merged. A similar argument holds for the steel mass and steel temperature. It can be seen in Table 4.1 that the average performance for the percentage carbon and off-gas temperature under manual control is $4.03 \%$ and the average performance for the steel mass and steel temperature under manual control is $2.9 \% . \overline{\Delta C}_{c v}$ is $4.25 \%$ under MPC control and $10.38 \%$ under manual control. The saving $\overline{\Delta C}_{c v}$ of MPC control over manual control in this 
work is therefore $6.14 \%$ compared to $6.37 \%$ in 'Assessment 2'. The difference of $\overline{\Delta C}_{c v}$ of two assessments is $0.23 \%$. This result shows that considering the dependence of CVs results in a decreased economic performance estimate, which is to be expected.

Table 4.1: Summary of assessment.

\begin{tabular}{|c||c|c|c||c|c|c|c|}
\hline \multicolumn{4}{|c||}{ Assessment 1 } & \multicolumn{3}{c|}{ Assessment 2, modified from Oosthuizen et al. (2004) } \\
\hline & Manual & MPC & Difference & & Manual & MPC & Difference \\
\hline$\overline{\Delta C}_{t_{o g}, c}$ & $4.03 \%$ & $0.00 \%$ & \multirow{2}{*}{$4.03 \%$} & $\overline{\Delta C}_{c}$ & $1.48 \%$ & $0.00 \%$ & $1.48 \%$ \\
\cline { 5 - 7 } & & & $\overline{\Delta C}_{t_{o g}}$ & $3.00 \%$ & $0.00 \%$ & $3.00 \%$ \\
\hline$\overline{\Delta C}_{t_{s}, m}$ & $2.90 \%$ & $0.48 \%$ & \multirow{2}{*}{$2.42 \%$} & $\overline{\Delta C}_{m}$ & $0.00 \%$ & $0.48 \%$ & $-0.48 \%$ \\
\cline { 5 - 7 } & & & $\overline{\Delta C}_{t_{s}}$ & $2.69 \%$ & $0.00 \%$ & $2.69 \%$ \\
\hline$\overline{\Delta C}_{p}$ & $3.45 \%$ & $3.77 \%$ & $-0.32 \%$ & $\overline{\Delta C}_{p}$ & $3.45 \%$ & $3.77 \%$ & $-0.32 \%$ \\
\hline$\overline{\Delta C}_{d}$ & $0.00 \%$ & $0.00 \%$ & $0.00 \%$ & $\overline{\Delta C}_{d}$ & $0.00 \%$ & $0.00 \%$ & $0.00 \%$ \\
\hline \hline$\overline{\Delta C}_{c v}$ & $10.38 \%$ & $4.25 \%$ & $6.14 \%$ & $\overline{\Delta C}_{c v}$ & $10.62 \%$ & $4.25 \%$ & $6.37 \%$ \\
\hline \hline$\overline{\Delta C}$ & $10.45 \%$ & $3.51 \%$ & $\mathbf{6 . 9 4 \%}$ & $\overline{\Delta C}$ & $10.69 \%$ & $3.51 \%$ & $\mathbf{7 . 1 8 \%}$ \\
\hline
\end{tabular}

\subsubsection{Hypothesis test}

A $t$-test can be used to investigate if there is a significant difference between the means of two populations. A basic assumption of a standard $t$-test is that the variances of two samples are estimates of the same population variance. However, an $F$-test performed on the experiment data shows that the two sample variances of overall cost under manual and MPC control are considerably different, so the standard $t$-test cannot be used. A robust $t$-test (Fisher and Yates, 1953) is then conducted to examine the saving of overall cost. It takes the difference of variances into account, makes an estimation of pooled variance and then illustrates that the saving of average cost of an EAF operation can be expressed with the significance level of 0.05 , as shown in the following:

The null hypothesis $\left(H_{0}\right)$ and alternative hypothesis $\left(H_{1}\right)$ are:

$$
\begin{aligned}
& H_{0}: \mu_{M P C}=\mu_{m a n} \\
& H_{1}: \quad \mu_{M P C}<\mu_{m a n}
\end{aligned}
$$

where $\mu_{M P C}$ is the population mean of the average overall cost under MPC control and $\mu_{\text {man }}$ is the population mean of the average overall cost under manual control. The estimate of the pooled variance is:

$$
\begin{aligned}
s^{2} & =\frac{\left(n_{1}-1\right) s_{1}^{2}+\left(n_{2}-1\right) s_{2}^{2}}{n_{1}+n_{2}-2} \\
& =\frac{(203-1) 6.5408^{2}+(217-1) 1.5667^{2}}{203+217-2} \\
& =21.94
\end{aligned}
$$

where $s_{1}$ and $s_{2}$ are the standard deviations of the average overall operational cost for manual and MPC control respectively. Here, $n_{1}$ and $n_{2}$ are the sample sizes for manual and MPC control respectively. The test statistic, $t$, is calculated as follows: 


$$
\begin{aligned}
t & =\frac{\overline{\Delta C}_{\text {man }}-\overline{\Delta C}_{M P C}}{s \sqrt{1 / n_{1}+1 / n_{2}}} \\
& =\frac{10.45-3.51}{4.6840 \sqrt{1 / 203+1 / 217}} \\
& =15.17
\end{aligned}
$$

where $\overline{\Delta C}_{\text {man }}$ is the sample mean of the overall cost under manual control, and $\overline{\Delta C}_{M P C}$ is the sample mean of the overall cost under MPC control. The value of the statistic $t$ for the 0.05 significance level is 1.645. $H_{0}$ can therefore be rejected with and it is illustrated that there is a $6.94 \%$ saving of overall cost of MPC control compared to manual control.

\subsection{Conclusion}

In this chapter the development of the IPFs of the EAF is reviewed firstly and important ideas used for the development are abstracted. These ideas may help to establish general methods of forming PFs. The JPFs are then developed after investigating the multivariate nature of the economic influence of $\mathrm{CVs}$ and the dependence of these variables. A multivariate economic performance assessment of the MPC and manual control for the EAF is conducted afterward, using the structured JPFs, which shows that the MPC control provides better economic performance than manual control. Furthermore, it is illustrated that the dependence of CVs should be considered when performing economic assessment for control systems in order not to inflate the economic performance estimate. 


\section{Chapter 5}

\section{State-of-art of control and performance assessment of grinding mill circuits}

\subsection{Introduction}

Grinding mill circuits are the most energy- and cost-intensive unit processes in the mineral processing industry and therefore the study of the control systems for grinding circuits remains important (Ramasamy et al., 2005; Wills and Napier-Munn, 2006). Grinding circuit control objectives range from maintaining system stability to achieving maximum economic benefit. A survey of the current practice of process control and functional/economic performance assessment as applied to grinding mill circuits was conducted to capture industry guidelines and standards. A summary of the survey results is reported in Wei and Craig (2009c). The survey also highlights PFs that could be used for systematic EPA of grinding circuits. Questions were also intended to generate some ideas on how to develop PFs for EPA of control systems. The main survey results are elaborated on in this chapter. Furthermore, a number of publications on milling control are examined and contrasted to the survey results.

The organisation of this chapter is as follows: Firstly the process overview of grinding circuits is given in Section 5.2. Primary survey theory and relevant techniques are given in Section 5.3. The research methodology and the questionnaire design used in the survey study are given in Section 5.4. The main survey results are then elaborated on in Sections 5.5, 5.6, 5.7 and 5.8 in line with the questionnaire structure. Conclusions are given in Section 5.9.

\subsection{Process description of grinding mill circuits}

After being mined and then crushed, ore is fed into grinding circuits in which the ore particle size is reduced to a suitable level so that the mineral contained in the ore can be liberated economically in downstream concentration processes. Grinding is conducted in spinning cylindrical steel containers known as tumbling mills, as shown in Fig. 5.1. These mills consist of a charge of the grinding medium, moving freely inside the mill and comminuting the ore particles. The grinding medium can be steel rods, or balls, hard rock, or the ore itself. 


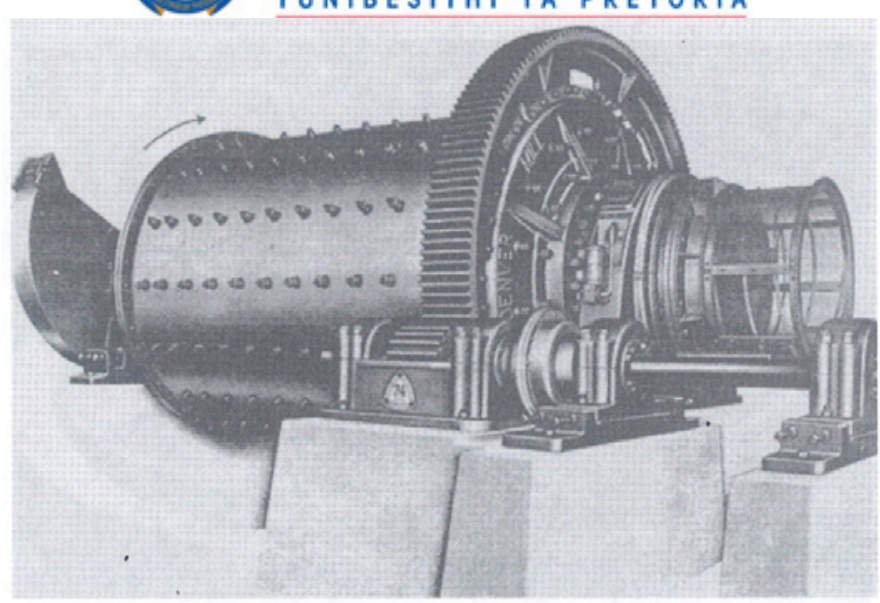

Figure 5.1: A typical tumbling mill (Wills and Napier-Munn, 2006)

All ores have an economically optimal mesh of grind, depending on various factors, e.g. the degree that the minerals are distributed in the gangue and the subsequent separation process. The purpose of grinding is to exert close control on the product size and thus proper grinding is often regarded as the centre of good mineral processing. Under-grinding of the ore will cause a too coarse product, with a degree of liberation too low for separation. Hence poor recovery will be result in the concentration stage. Over-grinding may reduce the ore particle size below the point required for most efficient separation. Whereas grinding is the most energy-intensive sector in mineral processing, finer grinding should not be conducted beyond the point where the net smelt return for the increased recovery becomes less than the added operating cost (Steane, 1976).

Grinding in a tumbling mill is affected by the size, quantity, type of motion and spaces between pieces of the medium in the mill (Wills and Napier-Munn, 2006). Grinding can take place in several mechanisms, i.e. impact or compression resulting from forces exerted on the particle surfaces, chipping resulting from oblique forces, and abrasion resulting from forces acting parallel to the surfaces. These mechanisms twist the particles and alter their shape beyond certain limits determined by their degree of elasticity, and causes them to break.

Grinding is usually conducted wet. When the mill is turned the mill charge (the mixture of grinding medium, ore and water) is blended and the grinding medium comminutes the particles using any of the methods mentioned above, depending on the speed of mill rotation. Most kinetic energy of the tumbling load is dispersed as heat, noise and other losses, and only a small part is used in breaking the particles. Grinding is a continuous process. Material is charged at a controlled speed from silos into one end of the mill and overflows at the other end after a time (Stanley, 1987).

Tumbling mills consist of three basic types: rod, ball and autogenous. Each type of mill comprises a horizontal cylindrical shell with renewable wearing liners. The drum is sustained in order to spin on its axis on hollow trunnions attached to the end walls. The feed is usually charged to the mill continuously through one end trunnion and the ground product leaves through the other trunnion. In some applications the product may be discharged through a number of ports around the periphery of the shell.

Grinding circuits may be open or closed, one-stage or multi-stage. In open circuit the material is charged into the mill at a calculated rate to yield the expected product in one pass. In 
closed circuit material of the required size is discharged by a classifier, which returns oversized material to the mill. Various types of classifiers can be used to close the circuit. A two-stage closed circuit is shown in Fig. 5.2 for illustrative purpose.

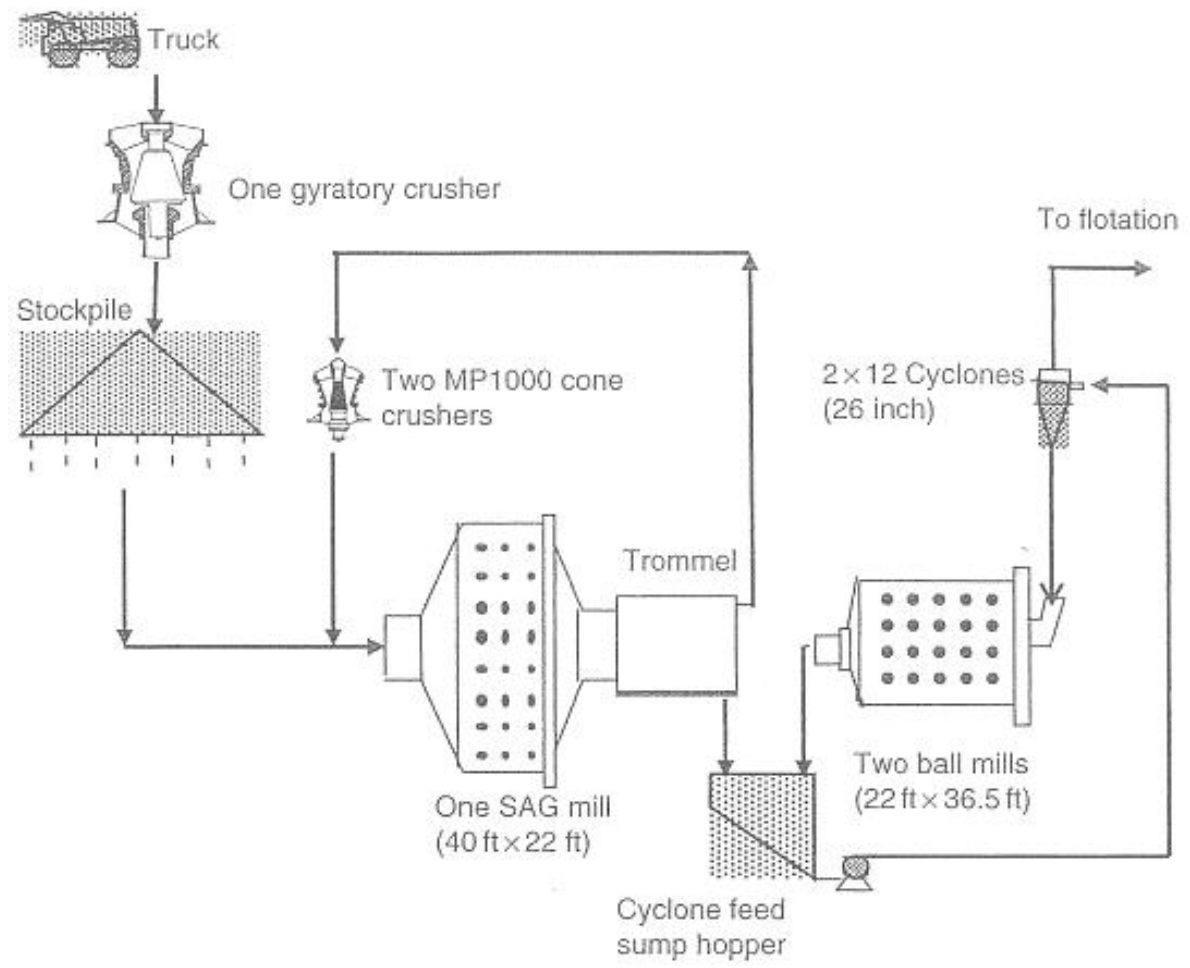

Figure 5.2: A two-stage closed circuit (Wills and Napier-Munn, 2006)

\subsection{Primary survey theory and techniques}

\subsubsection{An overview of survey research}

\subsubsection{The nature of survey research}

Survey research in the context used here concerns systematic observation or interviewing to describe a natural population and, usually, elicits inferences and causation or patterns of influence from the resulting data (Sapsford, 2007). A survey describes a population; its objective is to describe 'what is out there'. The word 'population', in statistical terminology, refers to the complete set of objects that one wishes to investigate. For survey research, the 'population' means the entire set on which one wishes to make generalisations. A clear definition of the population to which the results are to generalise is crucial in survey research and not always obvious.

\subsubsection{Main technologies for survey research}

Survey research involves three main technologies, i.e. measurement, sampling and comparison (Alreck and Settle, 1995). They are discussed below. 
Measurement: Different from other kinds of research projects, surveys involve systematic observation or interviewing. They ask the questions to which the research requires answers, and often specify the range of answers that may be given. Standardisation is the kernel of survey research, and the essence is to elicit consistent answers to consistent questions. The questions that need to be answered are asked in the same way to everyone. Moreover, the survey tries to ask questions in precisely the same manner in each interview, i.e. to standardise the questionnaire as a measuring instrument.

Sampling: A sample is a subset of the population, generally with the implication that the subset resembles the population closely in key characteristics and is thus representative of the population. If the sample is representative of the population, then what is true of the sample will also be considered true for the population, within a margin of error. The sampling pattern is not merely a simple random one, but a complicated stratified design that aims to produce a representation of the population that is as good as possible, including people in rare but important categories.

Comparison: Many surveys are not meant just to describe populations, but are designed to test some argument or at least to determine the difference between one group and another. Thus a paramount purpose of survey data is to make comparisons.

\subsubsection{Objective of the technologies}

The technical objective for all three of these 'technologies' is validation, establishing the validity of the research (Fowler, 2009). To ask whether a study is valid, or rather, the extent to which it is valid, is to investigate the status of the evidence. It is necessary to find out whether what is presented as evidence can carry the weight of conclusions drawn from it, or whether there is a logical defect (in measurement, sampling, or comparison) that makes the conclusion doubtful or detracts from belief in it. The reader needs a reason to trust these conclusions, and it is the responsibility of the survey report to provide good support for belief.

A research argument is considered to be valid when the conclusions follow from the data logically (Babbie, 1990). Questions required to test this involve:

1. Validity of measurement: the extent to which the data constitute accurate measurements of what is supposed to be measured.

2. Population validity: the extent to which the sample gives an accurate representation of the population it is intended to represent.

3. Validity of design: the extent to which the comparisons being made are appropriate to establish the arguments from the data.

\subsubsection{Developing ideas for survey research}

In this segment the crucial first stage of survey research, devising and developing an explicit research idea, which will facilitate obtaining valid, reliable, interpretable and useful results, is discussed. Firstly, the logical sequence of considering the research is discussed. Given the definition of the problem, obtaining valid evidence is discussed next. 


\subsubsection{The logical sequence of thinking and performing the research}

Research usually starts with a question or problem and proceeds to a solution based on the interpretation of evidence, which the examiner claims to be valid for the purpose (Czaja and Blair, 2005). What the problem or question is needs to be clarified in general terms first, and then stages of planning will take place with increasing precision. It is necessary to have some ideas about what kind of answer will be acceptable. Given these, it is important to propose an argument that will proceed from the problem to that kind of answer. Then it will be natural to determine what kind of evidence will be required and to plan the sampling and methods of collection so that the evidence can be obtained and its validity can be demonstrated to the reader. Thinking about what will be put into the final report can yield a list of questions to ask at the beginning:

What is the problem?

What kind of answer is required?

What kind of argument could proceed from the question to the answer?

What kind of evidence will sustain this kind of argument?

How is this kind of evidence to be procured, and from whom or about what?

How can it be demonstrated to the reader that the evidence is valid?

These problems remain valid for each kind of project.

\subsubsection{Validating evidence}

Given a sufficient definition of the problem, which means having at least a sound idea of the conclusions that the final report is intended to reach, the remainder of the planning stage concerns obtaining valid evidence for the conclusion and demonstrating its validity (Rea and Parker, 2005). It is necessary to consider the population from which the sample will be drawn, to avoid excluding cases that may be important for the argument. Another significant segment of survey design is to show that the measurements are accurate and trustworthy, i.e. someone else employing the same measurement would have achieved the same or similar results. Another equally important matter is to show that what one has measured does mean what it is intended to mean, that one has been successful in measuring what was needed for the argument.

An aspect of validation, reliability, means the stability of the measures, i.e. the degree to which repeated measurement provides constant results (over a fairly short period of time, during which change would not be anticipated to happen), or presumably identical measuring instruments lead to identical results. In the simplest cases, reliability is checked literally by measuring twice and looking at the agreement of the two measures.

\subsubsection{Advantages, importance and objectives of questionnaires}

\subsubsection{Advantages and importance of questionnaires}

As for the measurement of survey research, the first decision is whether to use an intervieweradministrated schedule or a questionnaire (Thomas and Lynn, 2007). The biggest advantage of self-completion questionnaires over structured interviews are their cheapness and the saving of the researcher's time, allowing much larger samples (Rea and Parker, 2005). Another advantage 
of self-completion questionnaires is that they are necessarily an entirely standardised measuring instrument; the questions are always phrased in exactly the same way for all respondents. The disadvantage is that the researcher cannot guarantee the amount or quality of attention that the respondent will give it. One is also not sure who actually fills it in, maybe the target respondent, maybe a committee of family or friend. These matters may greatly affect the validity of the information one receives.

The questionnaire represents one part of the survey process. It is, however, a very vital part of the process to ensure the success of data collection for survey research. The importance of structuring a good questionnaire is often underestimated. If the questions are worded incorrectly, phrased poorly, or in the wrong order, the answers obtained may be worse than meaningless: they may be misleading (Brace, 2004).

\subsubsection{Objectives in writing questionnaires}

The function of the questionnaire is to elicit the information that is required to enable the researcher to reach the objectives of the survey. In order to realise this, the questionnaire must not only collect the data required, but do this in as accurate a way as possible (Czaja and Blair, 2005). Collecting accurate data means obtaining the most accurate responses, thus a crucial objective in writing the questionnaire is helping the respondents provide them.

The first task of any questionnaire is defining the objectives that the study is expected to achieve. This will concern the issues at hand and may be very specific. When the objectives are specific, the questionnaire writer's task is usually rather more straightforward than when the survey is exploratory in nature. A concrete objective generally implies that there is a specific question to answer and it is the questionnaire writer's work to find out the most appropriate way of answering that question. Where research is exploratory the questionnaire writer's task is less predetermined, and an important part of the task is determining what data need to be collected and how these could be collected best.

\subsubsection{Designing questionnaires}

In this section the techniques of designing questionnaires are elaborated on. Firstly, general guidelines are given. The relevant detailed methods are discussed separately in turn, focusing on the different aspects of the design.

\subsubsection{General guidelines for designing questionnaires}

A questionnaire that can provide accurate, good-quality information needs to be meditated on and planned, before any single question is written down. The sequence of the different subjects that might be included in the questionnaire, the sequence of the different questions and the sequence in which prompted responses are given can all significantly influence the accuracy and reliability of the data received. It is also of primary importance to devise the questionnaire carefully to ensure that all respondents are asked the questions that they should be asked and not asked questions that do not concern them (Czaja and Blair, 2005). 
From the research objectives, it should be clear what information needs to be obtained. The required level of detail should also be apparent from the research objectives. Once the researcher determines the definition of the research scope, the data collection instrument and the survey design, the questions themselves can be drafted (Hague, 1993). The steps in devising questions comprise:

1. Specify the principal information required.

2. Clarify what else is demanded for analysis purposes.

3. Map the sequence of the subject scopes or sub-sections within the questionnaire.

The questionnaire writer should ask the questions pertinent to the objectives rather than be tempted to seek information that might be interesting but irrelevant, which could waste the resources of people involved, specifically the respondents.

\subsubsection{Further tips for structuring the questionnaire}

The questionnaire can be properly designed when the principal information requirements have been determined (Sapsford, 2007). It is most commonly divided into three sections: exclusion or security questions, screening questions and the main questionnaire. Exclusion is performed to ensure the confidentiality of the content of the questionnaire. Screening questions are used to choose the respondents for eligibility.

For the main questionnaire, the writer should conceive the order in which various topics are presented to the respondents. It is ideal to proceed from the most general topics to the most specific. If specific questions are asked first, then the respondents will be aware of the question writer's interest and this would bias their answers. Furthermore, starting with general topics allows the respondents to think about their behaviour before going into detail. There are some exceptions to this general rule when there is a good research reason for not starting with the more general questions, but the questionnaire writer should always be prepared to justify the decision.

It is important to design the questionnaire so that it flows smoothly from one subject area to another (Brace, 2004). Avoid returning to a preceding topic area. This makes the questionnaire look as if it has not been thought through and can confuse respondents who think they have dealt with this topic already. A flow diagram can help ensure that all topics are covered and the respondents are asked questions on the sections that are relevant to them.

Many things cannot be observed directly, and most surveys have at least some element of questioning informants and obtaining verbal answers (whether these be recorded by interviewers, written down by respondents or precoded as a choice of responses provided by the researcher). However, what is measured in this kind of research is not exactly what it is intended to be. Furthermore, a social desirability bias also arises sometimes, particularly when asking about attitudes or opinions. There are two other problems with attitude data, stemming from uncertainty about what kind of 'thing' an attitude is and what the nature of the person is who 'has' or 'holds' one.

Behaviour before attitude: It is generally sensible to commence any section of the questionnaire with behavioural questions before enquiring about attitudes and images. This partially allows the respondents to evaluate their behaviour and then to explain their behaviour through 
their attitudes.

Spontaneous before prompted: Considerable care must be taken not to prompt respondents with possible answers before asking questions mapped to get their spontaneous response.

Sensitive sections: If the questionnaire will involve questions of a sensitive nature, then these should not be asked at the beginning of the questionnaire.

Classification questions: Partially because they might be seen as intrusive, classification questions are usually asked at the end of the questionnaire. They are usually positioned there because they are normally disconnected from the subject matter of the questionnaire. Asking them earlier would disturb the flow of logic. The researcher needs to resist the temptation to ask for more classification data than are needed.

\subsubsection{Types of questions and data}

Questions can be asked and data recorded in various ways (Brace, 2004). Different types of questions are appropriate for different intentions and different types of data can be used and analysed differently. It is necessary for the questionnaire writer to understand the range of question types available, as the choice of question type will determine the information that will be elicited. It is also important to understand the different types of data that will be obtained, because that will determine the types of analysis that can be carried out. The researcher should thus be thinking about how to analyse the data when the questions are being framed so that the information collected can be analysed in the required way.

Any question in a questionnaire can be classified in several different ways (Sapsford, 2007):

1. Open or closed, depending on whether or not the answer will come only from a finite number of possible options.

2. Spontaneous or prompted, depending on whether respondents are requested to reply in their own words or given a number of options from which to choose an answer.

3. Open-ended or pre-coded, depending on whether the answer is recorded verbatim or against one or more of a number of predetermined options.

\subsubsection{Rating scales and attitude measurement}

The measurement of attitude is more difficult than that of behaviour (Hague, 1993). The answers to behavioural questions are only constrained by the respondents' memory of an event, the amount of effort they are willing to give to put into answering the questions and the degree to which they are prepared to be truthful. Respondents need help to express attitudes and describe images, especially in a manner that one can analyse. The most commonly used method of measuring attitude is the itemised rating scale. A rating scale is an interval scale on which respondents are requested to provide their answer, using a range of evenly spaced points, which are offered as prompts. Rating scales are popularly used in questionnaires. They offer a straightforward manner of acquiring attitude information that is easy and versatile to analyse, and that provides comparability across time. However, there are many different types of rating scales, and skill is necessary to choose the most appropriate one for an envisaged task. 


\subsubsection{Writing the questionnaire}

A number of issues need to be considered in writing the questionnaire (Brace, 2004), namely:

1. The language and style of language.

2. Assuring that there is no ambiguity in the questions and answers.

3. The use of pre-codes or responses that are recorded verbatim.

4. Bias that could result from the order of questions.

5. Bias that could result from the order of prompted responses.

The first part of the survey interview process is the briefing of interviewers to ensure standardised performance within a framework of natural behaviour. It is necessary to consider how the interview is to be managed, how to introduce the interviewer, how to clear up the survey's purpose and how to deal with sensitive questions.

People new to interviewing need to be trained to administer questionnaires in a standardised but sensitive manner, to avoid annoying or disturbing respondents and to establish rapport.

The wording of the questions needs to be precise, asking for exactly the information that is required. Questions should be unambiguous. Moreover, they must be as colloquial as possible, to be easily understood and to build the impression of a natural conversation instead of a profound checklist.

Routing is also important; where the interview goes next, depending on the answer to the question just asked (Czaja and Blair, 2005). Routing can be used to prevent asking questions repetitively, or in contradiction to what the respondent has just said, and therefore annoying him or her. It is also important that any routing instructions are clear, so that there is little chance of the interviewer leaving out questions that ought to have been asked.

Prompting is used to ask supplementary questions, trying to improve the quality of the answer (Fowler, 2009). Possible prompts can be used to favour a longer response. Prompting is an important section of survey work; it helps to obtain more than the bare immediate responses and gives more interpretable responses. It involves an inherent and unavoidable paradox, however. When one does prompt, one is not asking all informants the same questions, and the principle of standardization is breached.

As far as judgmental classifications are concerned, training and practice are of principal importance in guaranteeing reliability of measurement. It is crucial that the person making the judgment does so consistently, and that different judges classify the same things in the same manner.

\subsubsection{Ethical issues}

What is said in the introduction of a questionnaire is crucial in ensuring the cooperation of respondents (Brace, 2004). From an ethical standpoint the introduction should include:

1. The name of the organisation conducting the survey.

2. The broad subject area.

3. Whether the subject area is sensitive.

4. Whether the data collected will be held confidentially or used at a personally identifiable 
level for other purposes.

5. The possible length of the questionnaire.

6. Any cost to respondents.

7. Whether the results will be recorded.

\subsubsection{Social desirability bias}

However carefully the questionnaire writer constructs the questions, the data collected are only as accurate as the responses that are elicited. Respondents give inaccurate answers for different reasons. They give inaccurate answers both consciously for reasons of their own, and also without any conscious realisation that the information they give is inaccurate. The researchers must realise these inaccuracies, try to minimise them and, if necessary, take into account the bias and inaccuracy in the data (Brace, 2004).

Social desirability bias (SDB) occurs because respondents tend to appear to be different from what they are. This can occur consciously. The types of SDB include the following options:

1. Impression management: Probably the most common type of SDB is the need for approval, known as 'impression management'. Some people will not answer certain questions honestly when they feel the need for approval.

2. Ego defence and self-deception: Sustaining one's own esteem is another source of bias. Here respondents' intentions are not to manage the impression that they give to someone else, but to convince themselves that they think and act in socially responsible ways. When this is carried out consciously it is known 'ego defence'; when it is carried out subconsciously it is known as 'self-deception'.

3. Instrumentation: A further type of bias, which is totally conscious, is instrumentation. This means giving answers designed, in their own view, to bring about a socially desirable outcome.

When compiling the questionnaire, care must be taken to identify question realms that probably bring about SDB (Thomas and Lynn, 2007). If the questions enquire about attitudes or behaviour on any subject that has a social responsibility involvement, then consideration should be given to methods to minimise any possible bias. Only asking respondents to be honest has very little effect. Guarantees of confidentiality are not enough. Methods that are more helpful are required. These include the following:

1. Removing the interviewer.

2. Random response technique, which provides a platform for respondents to be honest on embarrassing or even illegal acts without the necessity for anyone to admit to such conduct.

3. Face-saving questions.

4. Indirect questioning.

\subsubsection{Sampling in the real world}

Some frequently used sampling methods other than random sampling are (Alreck and Settle, 1995):

1. Cluster sampling, where the researcher samples units or clusters, and maybe further clusters within them and then interviews or observes the entire population of the clusters or samples within it. The representation likelihood is increased if the number of clusters is large and if 
some level of randomisation is conducted at some or all of the section stages.

2. Quota sampling, where the researcher establishes a sample by deliberate selection of cases which will represent the population. The procedure is also improved if some degree of random selection can be used.

3. Haphazard sampling, as a sample of opportunity that happens to be accessible, cannot make a direct claim to represent the population, and relevant results should be interpreted carefully.

Statistical procedures are not formally valid if the sample is not selected randomly, but they are still used generally because although random sampling provides the highest probability of representing the population, it is often infeasible.

\subsubsection{Factors affecting the accuracy of collected data}

The collected data are supposed to be quite accurate. However, many problems are caused by problems within the questionnaire itself (Punch, 2003). These may include:

1. Ambiguity in the question.

2. Order influence between questions.

3. Order influence within a question.

4. Inadequate response coding.

5. Wrong questions asked owing to poor routing.

Some of the factors outside the direct control of the examiner in trying to obtain accurate and unbiased data comprise (Sapsford, 2007):

1. Questions asked in an inaccurate manner.

2. Failure of the respondent to understand the question.

3. Failure to record the reply accurately or completely.

4. Inattention to the interview from boredom and fatigue.

5. Inaccuracy of memory concerning behaviour.

6. Asking respondents to state attitudes on things to which they have no conscious attitudes.

7. Respondents lying as defiance.

8. Respondents intending to impress the interviewer.

9. Respondents unwilling to state their attitude or behaviour either consciously or subconsciously.

10. Respondents intending to influence the outcome of the investigation and providing answers they think will cause a specific conclusion.

\subsubsection{Tips on conducting interviews}

When interviewer-administrated interviews are conducted, an important issue is establishing rapport with the respondent, building a relaxed atmosphere in which even sensitive topics can be discussed, the truth is more likely to be obtained than deliberate deception and the respondent will give the most accurate and useful answers possible. Initial impressions are also crucial; the interviewer is supposed to maintain a good impression during the entire interview (Babbie, 1990). 


\subsection{Research methodology and questionnaire design}

\subsubsection{Objectives of and population for the survey research}

The objectives of the survey are to find out how grinding mill circuits are controlled and how crucial process variables are related to economic performance. The survey was conducted in English (language) and was aimed at experts on grinding mill circuits (population). Several channels were used to target the respondents, which increased the likelihood that relevant experts would be chosen.

\subsubsection{Sample selected in the survey research}

First, a contact list obtained from industry was used to send the survey to $\mathbf{8 5 0}$ relevant professionals by the end of April 2007. It was also sent to the $\mathbf{2 6 0 0}$ members of the South African Institute of Mining and Metallurgy. Furthermore, professionals who had published papers on the control of grinding mill circuits were contacted directly. The extensive search for respondents resulted in respondents being recruited from all over the world, as can be seen in Fig. 5.3.

\subsubsection{Questionnaire design}

\subsubsection{Coverage of the survey}

After the survey objectives are defined, the subject areas can be determined correspondingly. The questionnaire was designed to cover various aspects of grinding mill circuits. The first part identifies the respondents and the second part is aimed at obtaining background information on the circuits. The third part involves the choice of key process variables and their economic impact. Part four is concerned with the control of milling circuits and control loop performance and part five covers economic issues.

\subsubsection{Questions preparation}

Then, for each part of the survey, thought was given to choosing the data required, how it should be obtained, how to ask the questions, and what type of question needed to be used. Self-completion questionnaire was decided upon for the survey study. Only relevant questions were prepared.

Questions were posed depending on the specific information required and the possible responses. Some of the questions were posed as closed, prompted and pre-coded, e.g., the types of mills used. Since the currently available types of mills are limited to semi-autogenous, autogenous, rod, and ball mill, these four options are provided as options for mill types.

Some of the questions were prepared as open, spontaneous and open-ended, e.g., main problems 
confronted in the optimisation and control of grinding circuits. Because the problems confronted in different milling circuits are different due to various circuit configuration, control objectives, instrumentation and other factors, open answers are possible and open, spontaneous questions were designed accordingly.

Most questions were posed in close-ended responses with the supplement of spontaneous answers, which allows the respondent to choose an answer, to analyse the results in a straightforward manner and to provide additional valuable information.

The questions were all carefully structured to ensure precision and to maximise unambiguity. For example, when the methods for measuring product particle size are concerned, the nine proposed methods are all described in a concise and clear manner; each description is intended to facilitate the understanding of the method.

Routing was also designed to fit the thinking logic of the problems involved because rushing into the issues that are most focused on will usually not give respondents enough time to think about the problems from the most basic aspects to more specific questions and cannot elicit satisfactory answers. In the design of this questionnaire, general information on the milling circuit such as the circuit configuration, the produced element, the discharge method and so on is first involved, then somewhat more specific information, such as methods for measuring variables and methods for manipulating variables is concerned, followed by more specific information about the control issues, such as choice of process variables, control technology used, the considerations of choosing controlled variables etc. Afterwards, the information about functional/economic performance assessment is investigated. The routing realized the natural sequence of investigating important information, going from general to specific issues and from easy to difficult problems.

The design of the routing and questions ensured standardisation of the questionnaire and the survey research. Ethical issues were resolved at the beginning of the questionnaire, including the name of the organisation conducting the survey, whether the subject area was sensitive, the possible length of the questionnaire, any cost to respondents etc. A glossary at the end of the questionnaire explained the terminology used in the questionnaire.

\subsubsection{Conducting of the survey research}

The survey was carried out via a web-based questionnaire at http://millingsurvey.ee.up.ac.za and the distribution and collection of an MS Word document. The web platform was established using the PHP web development technique. It was first produced on the RedHat 9.0 operating system and then adapted to various web browsers and operating platforms. The results of the survey were stored in a MySql database. Data were collected from the middle of April 2007 to the middle of June 2007. In total, 68 responses were received. All responses were verified to be experts on grinding mill circuits, as can be seen from Fig. 5.4. The survey results should be interpreted with some caution in view of the relatively small sample size. The sample size does, however, compare favourably with that of Bauer and Craig (2008) and is considerably larger than some of the surveys discussed in Takatsu et al. (1998). 


\subsection{Survey results: Background information on grinding circuits}

This section gives background information on the milling circuits concerned, including mill types, liner shapes, lifter bars, mill discharge methods, number of milling circuits, circuit configurations, classification methods, minerals in the feed, grinding media, measured variables and methods for measuring different variables. The objective of this section is to put the remainder of the questionnaire into context.

\subsubsection{Basic background information}

Mill types Ball mills are used in around half of the plants (53\%); see Fig. 5.5. Semiautogenous/fully-autogenous (SAG/FAG) mills are used less frequently (38\%). Rod mills are used least often.

Liner shape The respondents were asked to specify the liner shape used in their circuits, the results of which are shown in Fig. 5.6. Wave liners are the most frequently used liners (31\%). Rib liners (24\%) and step liners (14\%) are used less frequently. The item 'Other' includes grid liners, integral lifters, lifters and backing plates, plates and lifter bars, and shell plates with loose lifters. It can be seen in this figure that none of the respondents uses Lorain liners.

Lifter bars Manganese and hard alloy steel are most commonly used for lifter bars, as indicated by $51 \%$ and $38 \%$ of the respondents respectively. Other materials used include chrome moly steel, hi chrome, manganese steel and rubber.

Mill discharge The respondents were asked to specify the mill discharge mechanism. Grates are mostly used (40\%), with trunnions used less frequently (22\%); see Fig. 5.7. Overflow discharge is less common.

Number of milling circuits Half of the respondents indicated that the number of milling circuits, in the mineral processing plant they work in, varies from one to three. There is often only one circuit in the plant (35\%). Only a few plants (10\%) have more than five circuits.

Circuit configuration The most common circuit configuration is a single-stage and closed circuit $(37 \%)$. The second most common configuration is two-stage, first an open and then a closed milling circuit (30\%). The 'Other' in Fig. 5.8 comprises single-stage open circuits and two-stage milling (first closed and then open circuit); multi-stage circuits are also available in some plants.

Classification methods for closed circuits The respondents were asked what was used for

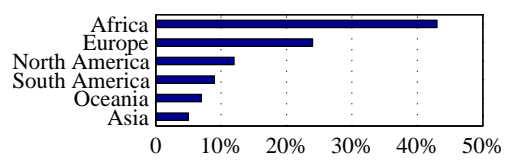

Figure 5.3: Continental distribution of the respondents 


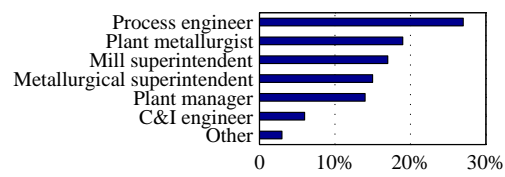

Figure 5.4: The positions of the respondents

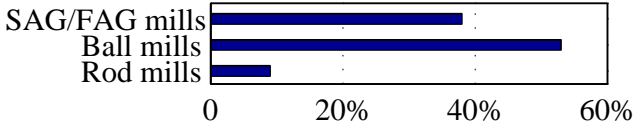

Figure 5.5: Mill types

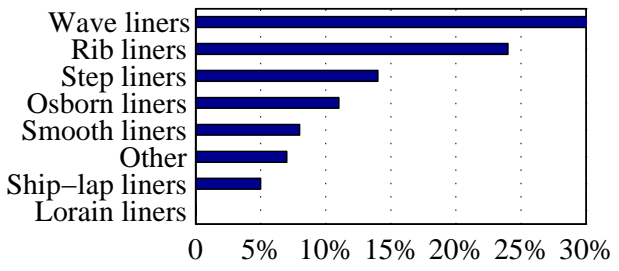

Figure 5.6: Liner shapes

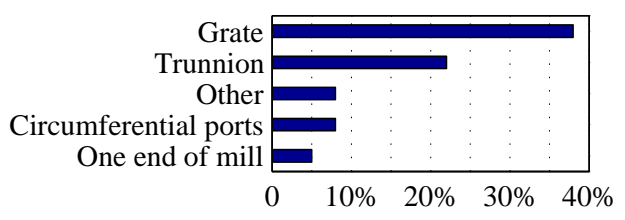

Figure 5.7: Mill discharge

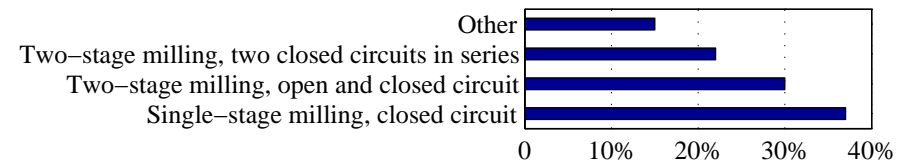

Figure 5.8: Circuit configuration 
classification if a stage of a circuit was closed. Cluster cyclones are mostly used (37\%), see Fig. 5.9. Single cyclones are less frequently used (27\%). The 'Other' classifiers include screw or rake classifiers and the combined use of screens and cyclones.

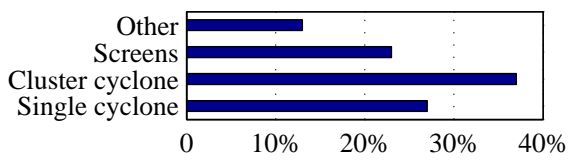

Figure 5.9: Classification instruments of a closed circuit

Minerals in the mill feed The minerals most frequently found in the mill feed are gold (27\%), platinum (22\%) and silver (19\%). The 'Other' in Fig. 5.10 includes cobalt, diamonds, lead, nickel, antimony, pyrite, magnetite, $\mathrm{Fe}, \mathrm{TiO}_{2}, \mathrm{Cr}_{2} \mathrm{O}_{3}$ and $\mathrm{CrFe}$.

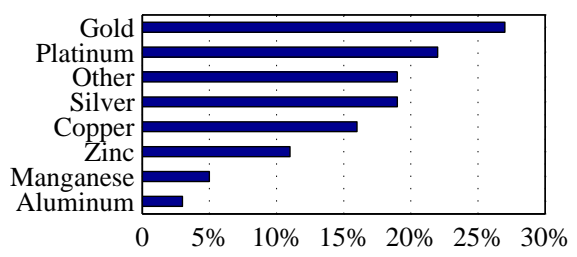

Figure 5.10: Minerals in the mill feed

Grinding media In most of the plants (92\%), balls are used as grinding media, see Fig. 5.11. Ore itself is used less frequently $(31 \%)$. The percentage profile of the grinding media corresponds to the types of mills.

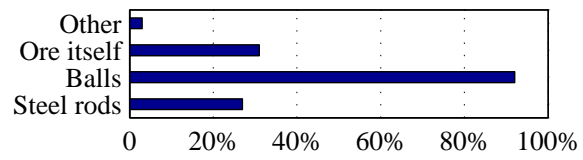

Figure 5.11: Grinding media

\subsubsection{Method for measuring variables}

Measured variables The respondents were asked to specify measured variables. These can be seen in Fig. 5.12. Mill power, feed rate of water to the mill, feed density of the sump discharge slurry and sump level are measured in over $80 \%$ of the milling circuits. The 'Other' in Fig. 5.12 comprises bearing pressure, bearing temperatures, cyclone feed pressure, mill ball charge, mill discharge density, mill speed and viscosity of slurry inside the mill.

Method for measuring product particle size The respondents were asked to specify the methods for measuring product particle size. Fig. 5.13 indicates that the most frequently used method is laboratory analysis (39\%). The 'Other' in Fig. 5.13 includes Mintek's PSE and ScanMin Ultra PSA (particle size analysis). Vision systems are implemented in FAG/SAG circuits to characterise the feed material, and the size distribution of material on a conveyor belt can be measured using image-based systems (Edwards et al., 2002). Product particle size is measured by using either on-line monitors or mathematical models (Wills and Napier-Munn, 2006). It is worth noting that Fig. 5.13 concerns only the respondents measuring this variable 


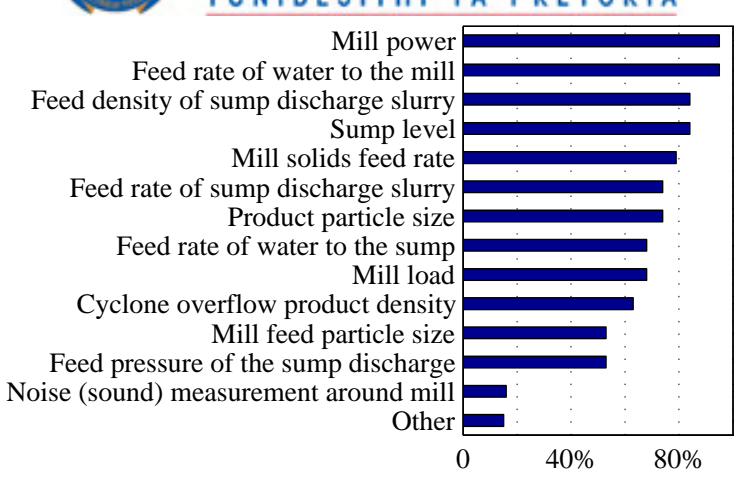

Figure 5.12: Measured variables

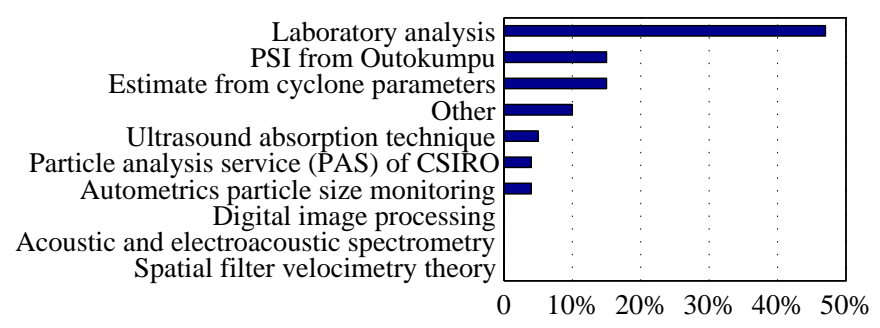

Figure 5.13: Method for measuring product particle size

instead of all the respondents. This is also the case for other measured variables discussed below.

Method for measuring mill load mass Fig. 5.14 shows the methods used for measuring mill load mass. Load cells are most frequently used (44\%). The mass can also be derived from bearing pressure (35\%). The 'Other' in Fig. 5.14 comprises a tape measure and Metscan mill vibration monitors. The popularity of using load cells for measuring the load mass is confirmed in Edwards et al. (2002) and Wills and Napier-Munn (2006). Load cells are strategically placed under the mill bearings and provide trustworthy signals. The advantage of load cells is that they measure absolute weight and the signal is independent of lube viscosity, temperature and other factors (Evans, 2001). These are most common on newer mills because retrofit costs can be high.

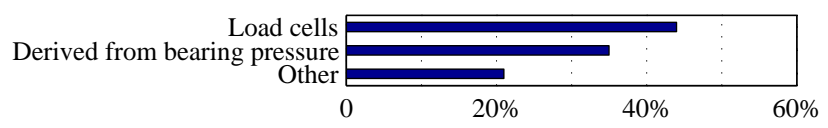

Figure 5.14: Method for measuring mass of mill load

Method for measuring mill load volume The methods for measuring the volume of the mill load are shown in Fig. 5.15. Dual microphones are often used (49\%). The 'Other' in Fig. 5.15 refers to power draw (Flook/Williamson), measuring the inside of the mill with a tape measure and measuring from a discharge lip. It is alleged in Edwards et al. (2002) that the volume is seldom measured, but is typically inferred from the power draw and weight of the mill by using a soft sensor. This statement makes sense, as it is impossible to measure the volume directly during normal operation. The measurement by dual microphones also depends on some inference based on the correlation between the volume and the intensity of the noise measured. Microphones have been found to have great advantages in measuring mill load volume (Wills and Napier-Munn, 2006). 
Figure 5.15: Method for measuring volume of mill load

Method for measuring sump level The ultrasonic technique is most frequently used (76\%) and the electrical technique (e.g. using the inherent conductivity of a liquid to vary the resistance seen by probes inserted into the material) is adopted less frequently (14\%). Pressure (using the relationship between the level and the pressure at the bottom of the vessel) and mechanical (using a float that is allowed to ride up and down as the level changes, which is connected by linkages to a secondary displacement measuring system) techniques are used occasionally (5\% each). Sienkiewica (2002) states that level measurements of liquids are usually conducted using sonic level sensors and transmitters. Sump level is often measured by bubble tube, capacitance-type detectors or other electronic devices (Wills and Napier-Munn, 2006).

Method for measuring sump discharge density Fig. 5.16 indicates the methods for measuring the density of the sump discharge. Nuclear density gauges are the most popular (75\%). The 'Other' in Fig. 5.16 comprises the Marcy density scale and specific gravity ( $\mathrm{Sg}$ ) scale. Edwards et al. (2002) confirm that nuclear density gauges are most frequently used for measuring slurry density in milling circuits, though differential pressure cells are sometimes used in sumps and classifiers. Sienkiewica (2002) confirms that nuclear density gauges are almost always used for density measurement. Wills and Napier-Munn (2006) also state that density is mainly measured with a nuclear density gauge.

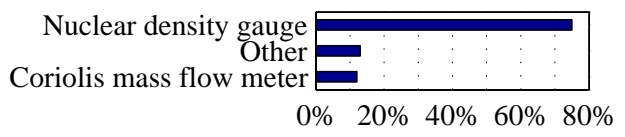

Figure 5.16: Method for measuring density of sump discharge

Method for measuring flow rates of water and slurry The respondents were asked to state the methods employed to measure the flow rate of water and slurry. Fig. 5.17 indicates that the magnetic flow meter is used most often (81\%). The 'Other' in Fig. 5.17 includes Doppler flow, wheel, tube and differential pressure based flow meters. It is confirmed in Edwards et al. (2002) and Wills and Napier-Munn (2006) that magnetic flow meters are widely used for measuring flow rates of water and slurry. They are usually trustworthy and need little maintenance. Sienkiewica (2002) states that electromagnetic flow meters are preferred for slurry and fluids containing suspended particles because they have the advantage of being obstructionless and having good turndown ratios.

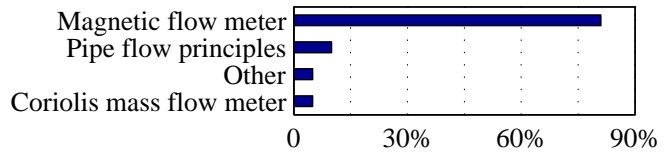

Figure 5.17: Method for measuring flow rate of water and slurry

In summary, the methods for measuring the flow rate of water and slurry, mill load mass, mill load volume, ore size and slurry density summarised in the survey results are in accord with Edwards et al. (2002). 
Method for measuring other variables Table 5.1 shows the methods for measuring variables other than those discussed above. The variables include mill power, mill solids feed rate, feed pressure of the sump discharge, cyclone overflow product density, mill feed particle size and acoustic measurements around the mill.

Table 5.1: Methods for measuring other variables

\begin{tabular}{|l|l|}
\hline Variables & Methods for measuring \\
\hline Mill power & Current transformers, Enermax, KWH meter, Mill motor KW output \\
\hline $\begin{array}{l}\text { Mill solids feed } \\
\text { rate }\end{array}$ & $\begin{array}{l}\text { (Idler) belt weightometer, load cell weightometer, weightometer coupled } \\
\text { with tachometer (UniPro by Precess Automation) }\end{array}$ \\
\hline $\begin{array}{l}\text { Feed pressure of } \\
\text { the sump dis- } \\
\text { charge }\end{array}$ & DPI (Druck pressure indicator), pressure gauge/transducer \\
\hline $\begin{array}{l}\text { Cyclone overflow } \\
\text { product density }\end{array}$ & $\begin{array}{l}\text { Automatic sampling, densitometer, hand sample and marcy scale, } \\
\text { Magflow, manual mass measurement, manual sample (use electronic } \\
\text { scale), Mintek PSE (particle size estimator), nuclear/coriolus (on bleed } \\
\text { stream or through launder), nuclear density gauge, Ramsey density meter }\end{array}$ \\
\hline $\begin{array}{l}\text { Mill feed particle } \\
\text { size }\end{array}$ & $\begin{array}{l}\text { Digital camera (Visiorock), laboratory wet screening, laboratory sieve, } \\
\text { manual screen analysis, PSI (particle size instrument) from Outokumpu, } \\
\text { tertiary screen bottom deck aperture opening, visual estimate }\end{array}$ \\
\hline $\begin{array}{l}\text { Acoustic mea- } \\
\text { surements } \\
\text { around mill }\end{array}$ & $\begin{array}{l}\text { Acoustic microphone, FFE (FLSmidth-Fuller Engineering) minerals SAG } \\
\text { analyser }\end{array}$ \\
\hline
\end{tabular}

\subsubsection{Actuators used for manipulating process variables}

The respondents were asked to state the methods for manipulating variables. The results are shown in Table 5.2.

Table 5.2: Methods for manipulating variables (\% is used to express the percentage of respondents using a particular actuator type)

\begin{tabular}{|l|l|}
\hline Variables & Actuator type \\
\hline $\begin{array}{l}\text { Feed rate of water to the } \\
\text { sump }\end{array}$ & Automatic control valve (98\%) \\
\hline $\begin{array}{l}\text { Feed rate of water to the } \\
\text { mill }\end{array}$ & Automatic control valve (97\%) \\
\hline $\begin{array}{l}\text { Feed rate of sump discharge } \\
\text { slurry }\end{array}$ & Variable-speed pump (95\%) \\
\hline $\begin{array}{l}\text { Feed rate of solids to the } \\
\text { mill }\end{array}$ & Variable-speed belt (42\%); vibratory feeders (52\%) \\
\hline $\begin{array}{l}\text { Cyclone cluster valve posi- } \\
\text { tion }\end{array}$ & $\begin{array}{l}\text { Remotely operated solenoid (88\%); Other (11\%): Automatic } \\
\text { control valve, manual control valve on surge tank }\end{array}$ \\
\hline Mill speed & $\begin{array}{l}\text { Electric motor (90\%); Other (9\%): Fixed speed, liquid resis- } \\
\text { tance starter (LRS), motor/gearbox and pinion arrangement }\end{array}$ \\
\hline
\end{tabular}




\subsubsection{Other background information}

Troublesome instruments and reasons The respondents were asked to specify which instruments are troublesome and why. Table 5.3 shows the results. The factors leading to trouble are mainly poor quality of the instrument, a poor environment for the device, outdated software and high maintenance costs.

Table 5.3: Troublesome instruments and reasons

\begin{tabular}{|l|l|}
\hline Instruments & Reasons \\
\hline Control valves & Wrong position in line \\
\hline Flow meters & $\begin{array}{l}\text { Poor quality; continually drift and require recali- } \\
\text { bration }\end{array}$ \\
\hline Level sensor & $\begin{array}{l}\text { Steam and slurry build-up in front of measuring } \\
\text { device }\end{array}$ \\
\hline Load cells below mill & Poor reliability \\
\hline Densitometers & Continually drift and require recalibration \\
\hline Pressure transducers & $\begin{array}{l}\text { Cementation on sensing parts, choking of offtake } \\
\text { pipe }\end{array}$ \\
\hline $\begin{array}{l}\text { Particle size distribution moni- } \\
\text { tors }\end{array}$ & High maintenance cost \\
\hline Particle size instrument & Outdated software, expensive to replace \\
\hline Valves, flowmeters & Poor quality water \\
\hline Vibrating sensors & Poor quality \\
\hline
\end{tabular}

Instrument wish list The respondents were requested to specify what instruments, in addition to those currently installed, they would like to have, and what variables these should measure or manipulate. The results are shown in Table 5.4. Various instruments are desired at different grinding circuits. Some instruments wanted in one grinding mill circuit are used in others. It can also be seen from the table that more measurement instruments are wanted than actuators.

Table 5.4: Instrument wish list

\begin{tabular}{|l|l|}
\hline Measured variables & Instruments \\
\hline Cyclone O/F or U/F density & Densitometer, nuclear density gauge \\
\hline Cyclone U/F flowrate & Flowmeter \\
\hline Feed size to mill & Digital image processing \\
\hline Mill density & Nuclear density guages \\
\hline Mill load & Load cells \\
\hline Mill volumetric filling & $\begin{array}{l}\text { Impact meter to determine the location of impact } \\
\text { on the shell }\end{array}$ \\
\hline Particle size & $\begin{array}{l}\text { Particle size monitor, online analyzer, ScanMin Ul- } \\
\text { traPSA }\end{array}$ \\
\hline Power & Power meter \\
\hline Steel ball consumption & Ball addition equipment \\
\hline $\begin{array}{l}\text { Estimation of point of impact of } \\
\text { charge }\end{array}$ & Dual microphone \\
\hline Variables manipulated & Actuators \\
\hline Mill motor speed & Variable frequency drive (VFD) motor \\
\hline
\end{tabular}

Grinding media for semi-autogenous milling The amount of grinding media (steel balls) added per metric ton of ore milled for semi-autogenous milling varies from 300 to $1250 \mathrm{~g}$. 


\subsection{Survey results: Choice of crucial process variables and their economic impact}

The purpose of milling is to reduce the particle size such that liberation of valuable minerals can take place. Because the sump level and mill load are usually open loop unstable, they need to be controlled to stabilise the system. Considering the purpose of grinding mill circuits, the product particle size is usually controlled. Stabilising control is often realised with single loop controllers. A supervisory control system can then automatically regulate the setpoints for the stabilising control loops when the ore quality changes in order to achieve optimal circuit performance (Samskog et al., 1996; Borell et al., 1996). The relevant control objectives may be (Mular, 1989; Craig and Macleod, 1995; Wills and Napier-Munn, 2006):

1. to improve the quality of the product, i.e. increasing the particle fineness and decreasing the particle size fluctuations.

2. to maximize the throughput.

3. to decrease the power consumption.

4. to reduce the usage of the grinding media (e.g. steel ball) for some circuits.

The control objectives are interrelated and usually a trade-off needs to be made. A circuit may occasionally be controlled at constant production rate, trying to maximise the product fineness, but most of the time it is controlled at a fixed product particle size setpoint, whilst trying to maximise the throughput (Hodouin et al., 2001). Maximisation is usually achieved by keeping the circulating load constant, but it can also be done using real-time optimisation strategies (Wills and Napier-Munn, 2006).

This section of the survey enquires about what variables are chosen for the control of grinding circuits and considerations for chosen CVs. It firstly involves the choice of CVs, manipulated variables. For every CV, considerations for choosing a setpoint, corrective action taken when the variable deviates from the setpoint and the resultant economic impact are taken into account. The dependence of CVs is also investigated.

\subsubsection{Choice of key process variables}

\subsubsection{Choice of controlled variables}

The respondents were asked to specify which variables are controlled most often. It can be seen from Fig. 5.18 that the product particle size, slurry level in the sump and sump discharge slurry density are the three CVs used most frequently. One would expect some form of control for the sump level and the mill load, as these variables are open loop unstable. In Muller et al. (2003), CVs include the particle size, sump discharge slurry density, sump level, mill load and mill power; the mill load and sump level are controlled to ensure stability, whereas the particle size is controlled to maintain a fixed setpoint with as little variation as possible and mill power is controlled to optimise the throughput. In Hulbert et al. (1990) and Craig and Macleod $(1995,1996)$ the mill load and sump level are also controlled to stabilise the circuit. In addition, particle size is also controlled. In Ramasamy et al. (2005), however, besides particle size, the throughput is also controlled. It is important to note that independent particle size and throughput control is not possible. 
Not all the respondents chose all the variables given in Fig. 5.18. In the following questions concerning each CV (Sections 5.6.2 to 5.6.5), the responses are only for respondents who selected that variable as a CV.

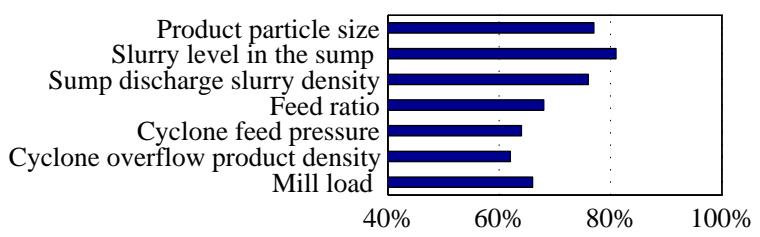

Figure 5.18: Choice of CVs

\subsubsection{Choice of manipulated variables}

Fig. 5.19 shows the choice of manipulated variables. The flow rate of water to the sump, flow rate of water to the mill, feed rate of solids to the mill and flow rate of slurry from the sump are all frequently adopted as manipulated variables. Hodouin et al. (2001) declare that the solid feed rate and water addition rate to the sump are the manipulated variables used most often. In Muller et al. (2003) the four variables mentioned above are used to control the milling circuit. In Hulbert et al. (1990) the feed rate of water to the sump, the flow rate of sump discharge slurry and the feed rate of solids to the mill are manipulated; based on Hulbert et al. (1990), the flow rate of water to the mill is used to expand the range of particle size control (Craig et al., 1992a). The solid feed rate to the mill and the flow rate of water to the sump are controlled in Ivezić and Petrović (2003); Niemi et al. (1995); Pomerleau et al. (2000). In Ramasamy et al. (2005), the solid feed rate to the mill and flow rate of water to the sump are used as manipulated variables. The flow rate of water to the sump is manipulated in Yahmedi et al. (1998).

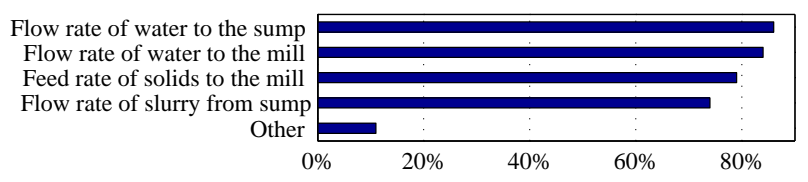

Figure 5.19: Choice of manipulated variables

\subsubsection{Setpoint choice, corrective action and the economic impact of the product particle size}

The respondents were asked to state the considerations when determining the setpoint for the product particle size. The results are shown in Fig. 5.20. The efficiency of the downstream process, e.g. leaching or flotation, is of primary importance $(89 \%)$, which is confirmed by Hulbert (2002), Wills and Napier-Munn (2006) and Hodouin et al. (2001). Circuit throughput is also an important factor (53\%). In addition, energy consumption is regarded as an important factor in Hodouin et al. (2001) and Wills and Napier-Munn (2006). The 'Other' factor in Fig. 5.20 includes best overall economic performance (which can be very complex) and capacity.

Fig. 5.21 indicates the actions taken to grind finer. The two main actions are a decrease in the feed rate of solids to the mill and an increase in the flow rate of water to the sump. The 
Figure 5.20: Considerations when choosing setpoint for product particle size

'Other' actions in Fig. 5.21 comprise automated ball loading, reviewing cyclone internal parts to suit a finer cut size, increasing mill power, increasing the grinding energy by increasing the steel charge and reducing the steel ball size, increasing mill speed and reducing the mill load. These actions are mainly performed manually (62\%).

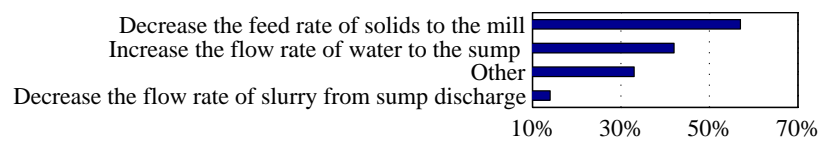

Figure 5.21: Action taken to grind finer

The results of actions taken to grind coarser are shown in Fig. 5.22. Two main methods are to increase the feed rate of solids to the mill and to decrease the flow rate of water to the sump. The 'Other' in Fig. 5.22 includes changing the cyclone spigot size, increasing the mill load, reducing mill speed, decreasing the grinding energy in the milling by reducing the steel charge and using larger steel balls.

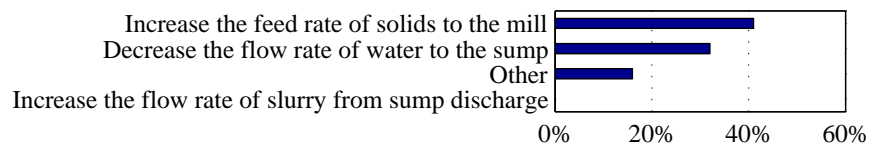

Figure 5.22: Action taken to grind coarser

The qualitative economic impact of grinding finer than the setpoint is shown in Fig. 5.23. Better extraction downstream and reduced throughput are the most common impacts. The label 'Other' in Fig. 5.23 comprises decreased concentrate grade, depending on where one is on the grind recovery curve, higher metal recovery from downstream flotation circuits and a possible decrease in process efficiency. Grinding coarser than the setpoint has the opposite impact.

\subsubsection{Setpoint choice, corrective action and the economic impact of the sump level}

As for the considerations when choosing the setpoint for the sump level, the stability of the system is the first priority (78\%). Maintaining a margin of safety is the second choice $(67 \%)$.

The most frequent action taken when the sump level is too low, is to increase the feed rate of water to the sump (47\%). Reducing the feed rate of slurry from the sump discharge also increases the sump level (39\%). Troubleshooting as corrective action is also conducted in a few cases. When the sump level is too high, reducing the feed rate of water to the sump is the first priority (44\%); see Fig. 5.24. The other two frequently used methods are to increase the feed rate of the sump discharge slurry and to investigate whether the controller runs normally. The item 'Other' indicates checking pump speed and duty and checking the efficiency of the 


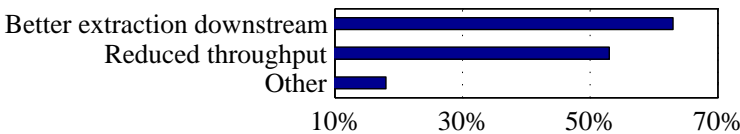

Figure 5.23: Qualitative economic impact of grinding finer than expected

discharge pump; if the slurry level is not critically high, the sump controller can also be used to reject a disturbance. These actions are mainly taken automatically (83\%). The controller in Hulbert (1989) acts on the square of difference between the sump level setpoint and the sump level (also known as error square control); when the deviation is small, less action is taken and when the deviation is big, more action is taken.

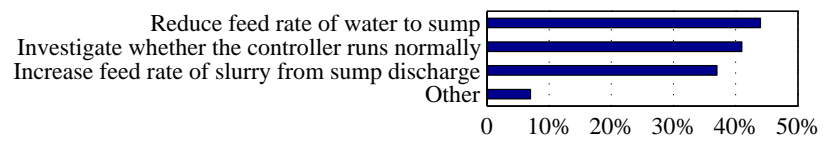

Figure 5.24: Corrective action for too high a sump level

The qualitative economic impact of a sump level deviating from the setpoint is shown in Fig. 5.25 and Fig. 5.26. When the sump level is higher than the setpoint, most (94\%) of the respondents indicate that spilled slurry needs to be handled. When the sump level is lower than the setpoint, the largest percentage of the respondents (44\%) state it will affect normal operation adversely. The item 'Other' in Fig. 5.25 includes unacceptable high-grade spillage on the mill floor (loss of valuable minerals in the form of spillage) and the potential for theft. The impact of 'Other' in Fig. 5.26 comprises the following:

- Discharge pump will surge (high pump maintenance).

- Cyclone feed pressure will go down (coarser grind).

- Instability.

- Pump cavitation, high wear and maintenance on pump/pipe and classifier system.

- Poor system stability, reduced efficiency and increased cost.

- Reduced stability due to flow variations.

- Poor cyclone performance.

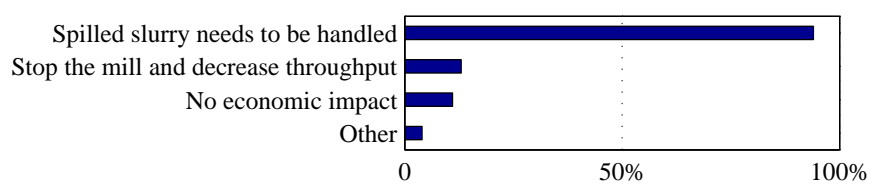

Figure 5.25: Economic impact of too high sump level

\subsubsection{Setpoint choice, corrective action and the economic impact of the mill load}

The efficiency of power consumption is the first priority (80\%) when considering the choice of the setpoint of the mill load. The stability of the system is also regarded as an important factor $(67 \%)$. Avoiding stoppage of the operation was chosen by $14 \%$ of the respondents.

The most frequently taken action when a mill load is too low, is to increase the feed rate of solids to the mill $(73 \%)$. In some milling circuits, the mill speed is decreased (29\%) or the addition of 


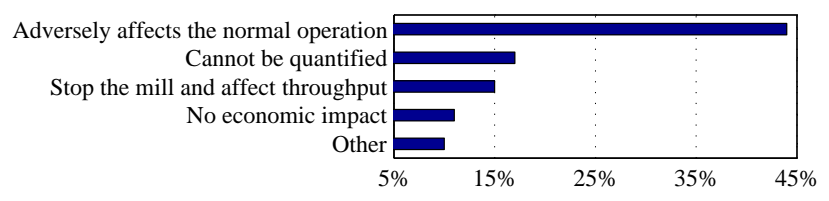

Figure 5.26: Economic impact of too low sump level

water to the mill feed is reduced $(27 \%)$. When the mill load is too high, the opposite actions are taken.

The qualitative economic impact of a mill load deviating from the setpoint can be seen in Fig. 5.27 and 5.28. When the mill load is too high (see Fig. 5.27), stoppage of the mill to reduce the load, thus interrupting production, is chosen by $47 \%$ of the respondents. Additional manpower required to reduce the mill load manually is taken into account by $22 \%$ of the respondents. The item 'Other' in Fig. 5.27 includes reduced efficiency (high power consumption, but little production) and reduced throughput and therefore reduced revenue.

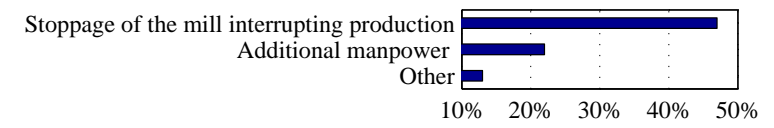

Figure 5.27: Economic impact of too high a mill load

When the mill load is too low, the largest percentage of the respondents (87\%) indicated it would lead to excessive ball-on-liner contact and therefore damage the mill liners. Consumption of steel and decreased throughput are both regarded as serious consequences. Approximately half of the respondents stated it would result in waste of power.

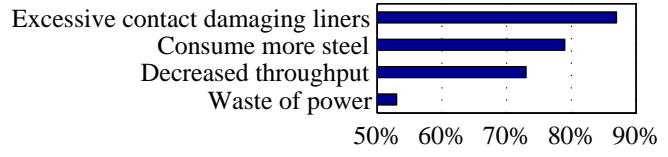

Figure 5.28: Economic impact of too low a mill load

\subsubsection{Setpoint choice, corrective action and the economic impact of the sump discharge slurry density}

The stability of the system is the first priority when determining the setpoint of the sump discharge slurry density (53\%). Almost half of the respondents (47\%) regard the smooth running of the system as important. Avoiding cyclone choking is also considered significant by $41 \%$ of the respondents. The fineness of the product is regarded as an important factor by $18 \%$ of the respondents.

When the sump discharge slurry density is too low, the three actions taken most frequently are decreasing the flow rate of water to the sump, increasing the feed rate of solids to the mill and reducing the flow rate of water to the mill; see Fig. 5.29. When it is too high, the opposite steps are taken. These actions are primarily taken automatically $(65 \%)$. 
Figure 5.29: Corrective action for a too low sump discharge slurry density

The qualitative economic impact of the sump discharge slurry density deviating from the setpoint is now considered. When the density is too high, downstream process inefficiency and decreased production are regarded as the most important consequences by the largest percentage of the respondents (chosen by $73 \%$ and $53 \%$ of the respondents respectively). When the density is too low, the impact is primarily the potential wastage of reagents in downstream processing (possibly due to increased surface area) and losses (possibly due to lower recoveries for the finer fractions) in recovery $(76 \%)$.

\subsubsection{Performance functions of the controlled variables}

The respondents were asked to specify the relationships between the CVs and the relevant economic impact. Several sample PFs are provided in Fig. 5.30 that are used frequently for economic assessment of control systems (Bauer et al., 2007). Option 4 can be found in Craig et al. (1992b) to relate residue (in terms of gold not recovered) and product particle size. The respondents could also come up with their own relationships. The $y$ coordinate is used to represent revenue or cost, and the $x$ coordinate represents the CVs.

The results are shown in Fig. 5.31. It can be seen that the majority of the respondents selected options describing the economic impact of the mill load (74\%), but only one-third did so for the sump discharge slurry density (36\%). $68 \%$ of the respondents indicated the economic impact of the product particle size, and $50 \%$ of the respondents did so for the impact of the sump level. For all the CVs in Fig. 5.31, more than one figure was selected to describe the economic impact.

Different figures were chosen, as the definition of monetary value differs. For example, for product particle size, options (a), (b), (c) and (d) are chosen to express it. The physical meaning of the $y$ axis for option (a) was not specified by the respondents and for option (b) the meaning was specified as 'increase in recovery'. For option (c), the meaning was regarded as 'metal revenue' or 'metal recovery'. For option (d), the meaning was either cost or revenue. This means that when considering the economic impact of one $\mathrm{CV}$, the type of PF may depend

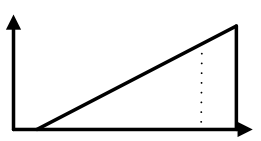

(a) Option 1

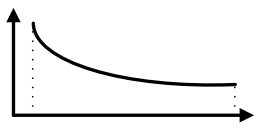

(d) Option 4

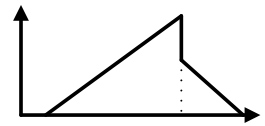

(b) Option 2

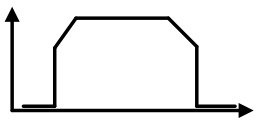

(e) Option 5

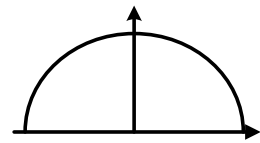

(c) Option 3

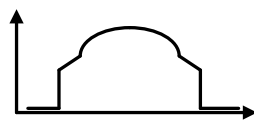

(f) Option 6

Figure 5.30: The options of PFs 


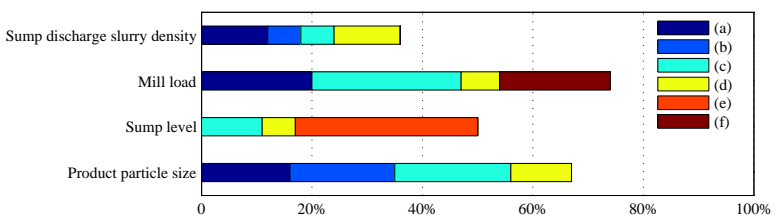

Figure 5.31: Economic impact of the CVs deviating from the setpoints. The (a), (b), (c), (d), (e), and (f) here refer to the subplots in Fig. 5.30 respectively

on how the revenue or cost is defined for particular milling circuits.

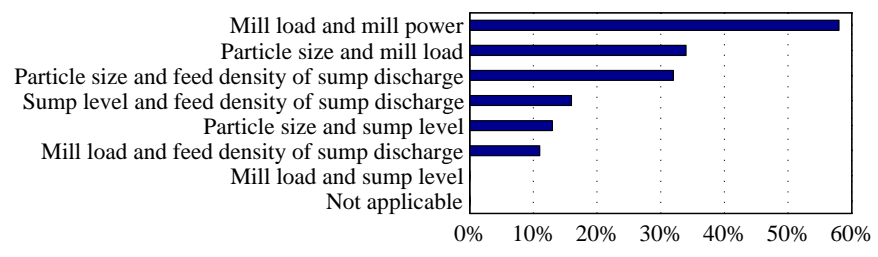

Figure 5.32: Dependence of CVs

\subsubsection{Dependence of controlled variables}

The respondents were asked to specify which variables are dependent when the control system is in place. When the multivariate nature of a system is considered, PFs of the individual CVs can become JPFs, which can lead to a more accurate estimation of the system's economic performance (Wei et al., 2007). (From the viewpoint of probability theory, random variables are independent when their JPDF is the product of the IPDFs (Evans and Rosenthal, 2004).) The results are shown in Fig. 5.32. The mill load and the mill power are regarded as dependent by over half of the respondents (58\%). Another two pairs of variables that are regarded as dependent by a considerable percentage of respondents are the particle size and the mill load, and the particle size and the density of the sump discharge slurry. However, in Craig and Macleod (1995) the frequency domain model of a ROM milling circuit involves three input variables (feed rate of solids to the mill, feed rate of water to the sump, and feed rate of slurry from the sump) and three output variables (product particle size, slurry level in the sump and mill load mass). The product particle size and mill load mass are affected by all three input variables, but the sump level is only affected by the feed rate of water to the sump and the feed rate of slurry from the sump. All three output variables are thus dependent in this milling circuit. One could however separate mill load control from that of sump level and particle size, as time constants differ significantly (Coetzee et al., 2009).

\subsection{Survey results: Functional performance assessment of grinding circuits}

This section discusses the frequency of control system performance monitoring, satisfaction with the control loop performance and problems confronted in the control and optimisation of grinding mill circuits. 


\subsubsection{Frequency of monitoring the control loop performance}

In most grinding mill circuits, control loop performance is monitored frequently (online: 40\%, daily: $32 \%$ ). Weekly monitoring is mentioned by $18 \%$ of the respondents. There is basically no mill circuit without control loop monitoring, which is in line with recent trends (Jelali, 2006).

\subsubsection{Satisfaction with control loop performance}

Only a few of the respondents are completely satisfied with the control loop performance (8\%). A significant percentage of the respondents (38\%) indicated that there was room for performance improvement. This could be due to the fact that PID control is used predominantly for a process that is inherently multivariable.

\subsubsection{Main problems confronted in the control or optimisation of the grinding mill circuit}

Various challenges exist in the control or optimisation of grinding mill circuits (Hodouin et al., 2001; Louw et al., 2003). Some respondents in the survey stated the following challenges:

- The control of sump discharge slurry density

- Mill sump level control

- Cyclone feed pressure control

- Product particle size control

- Mill discharge viscosity control

- Mill load control

- Methods to determine the power drawn for the mill

- Robustness of the system to feed ore hardness variations

- Automated steel ball loading.

The lack of on-line process information is regarded as the main problem and is highlighted by Galán et al. (2002). Mill optimal power determination for maximum throughput and solids feed control are considered significant issues by Louw et al. (2003).

\subsection{Survey results: Economic performance assessment of grinding mill circuits}

The economic assessment of grinding circuits is discussed, including cost/benefit types of control, methods for estimating cost/benefit and the importance and accuracy of, as well as satisfaction with, the economic analysis.

The economic performance of milling circuit control systems is often not satisfactorily quantified (Hulbert, 1989, 2002; Lo et al., 1996; Radhakrishnan, 1999; Bourassa et al., 1995) although the primary objective, when installing a control system, is to gain as much monetary benefit as possible. It was therefore decided to ask survey respondents about the EPA process as it relates to their installations. 


\subsubsection{Methods for estimating benefit}

As for the methods for estimating benefits before the control system is implemented, past experience with similar projects is used more often (86\%). Before control variance estimation is another frequently used method $(78 \%)$. When the control system is in place, benefits are estimated in most milling circuits (89\%) from experiments where respondents switch between the old and new control system, similar to what is described in Craig and Koch (2003). 17\% of the respondents indicated that only qualitative estimates were made.

\subsubsection{Cost estimation methods}

As far as the methods for estimating the cost of a control project for milling circuits are concerned, two frequently used methods are past experience with similar projects (91\%) and installation cost quoted by the vendor (83\%). This accords with the results of Bauer and Craig (2008) for process industries as a whole.

\subsubsection{Importance and accuracy of, and satisfaction with economic assessment}

The importance of economic analysis at different stages of the implementation of a control system is considered. Fig. 5.33 indicates that for half of the respondents cost/benefit analysis is indispensable before system implementation. After-control analysis is also important, but not as important as before-control analysis. This is in accordance with the result reported in Bauer and Craig (2008).

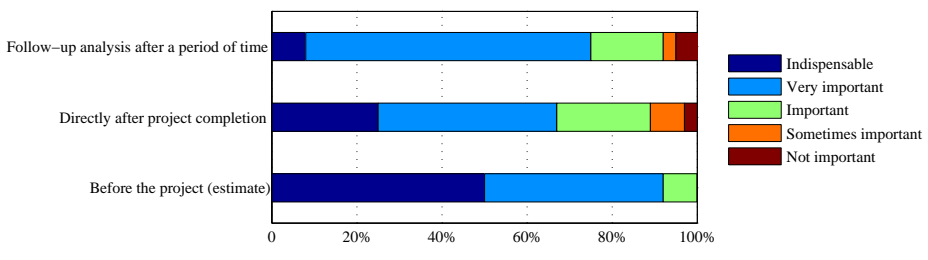

Figure 5.33: Importance of economic analysis

The respondents were asked to state how accurate the economic analysis should be at different stages of control system implementation; the relevant results are given in Fig. 5.34. The requirement of being as accurate as possible before system implementation is not as strong as directly after implementation. Overall, the economic analysis needs to be 'at least accurate' before and directly after implementation. This is in contrast to the results of Bauer and Craig (2008), where the follow-up analysis is supposed to be 'the most accurate'.

The respondents were asked to declare how satisfied they were with the current economic analysis techniques at different stages of the implementation of a control system, and the results are given in Fig. 5.35. Less than half of the respondents were satisfied with their cost/benefit analysis methods. Some respondents clearly expressed dissatisfaction with the analysis methods being used. This is in accord with the results of Bauer and Craig (2008) and highlights the potential for improving economic assessment techniques. 


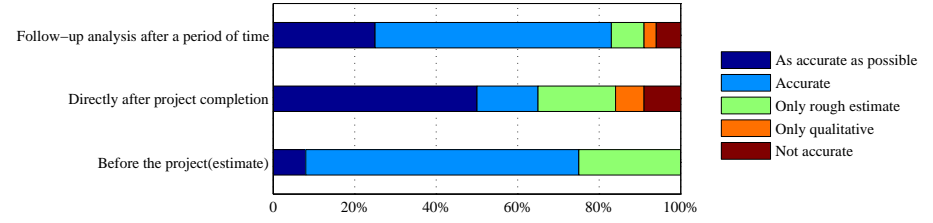

Figure 5.34: Accuracy of economic analysis

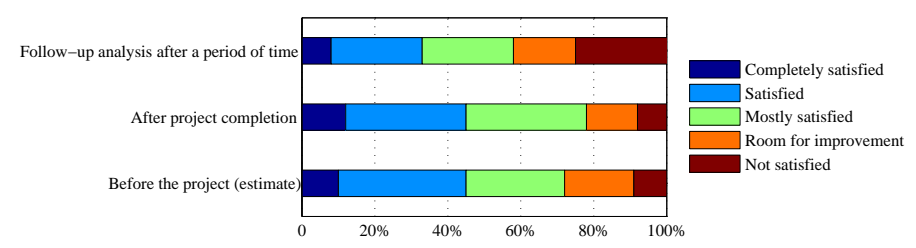

Figure 5.35: Satisfaction with economic analysis

\subsubsection{Most important topics requiring additional development}

The respondents were asked to specify the three most important topics requiring additional development. Fig. 5.36 shows that a lookup table as reference guide is the most popular (61\%). Combined analysis for process control and real time optimisation was regarded as important by a majority of the respondents (59\%). Base case benefit estimation from historical data was also selected by almost half of the respondents $(47 \%)$.

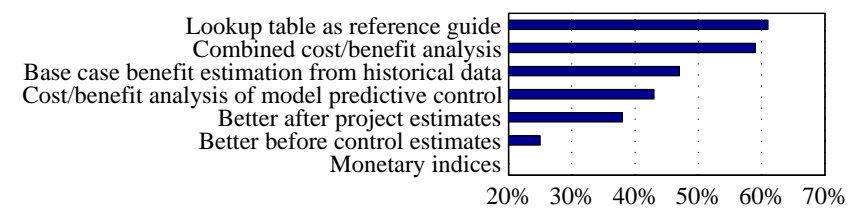

Figure 5.36: Topics requiring additional development

\subsection{Conclusion}

This work describes the results of a survey that was conducted to gauge the current status of control and functional/economic performance assessment of grinding mill circuits. A literature study on grinding mill circuit control was also conducted and the results were used to emphasise the survey results.

The main conclusions from the survey are as follows:

1. The three variables that are most frequently controlled are the product particle size, the slurry level in the sump and the sump discharge slurry density.

2. The most commonly used manipulated variables are the flow rate of water to the sump, the flow rate of water to the mill, the feed rate of solids to the mill and the flow rate of the sump discharge slurry.

3. The following are the main considerations when choosing setpoints for the CVs: the efficiency (liberation) of the downstream process (product particle size), system stability (sump level and sump discharge slurry density) and efficient power usage (mill load). Economic considerations 
are not explicitly stated.

4. There is usually some economic impact when the setpoints of CVs are violated.

5. Fewer than half of the respondents quantified the economic impact of the CVs deviating from their setpoints in terms of mathematical functions. Different mathematical functions were sometimes chosen for the same control variable. This implies that there is some confusion regarding the exact relationship between milling circuit CVs and money or perhaps that these relationships are different for every process.

6. More than half of the respondents indicated that CVs were dependent on each other. This is not surprising, as milling circuits are known to be candidates for multivariable control owing to the interaction of variables.

7. PID controllers are predominantly used in grinding mill plants, and more than half of the respondents are satisfied with the performance of their control loops. This fact tends to counter point 6 , and appears to imply that respondents are happy to live with the interactions that do exist.

8. Process control system benefits arise mainly from process stability improvements, an increase in throughput and a reduction in energy consumption. The most popular method used for estimating benefits is past experience with similar projects. The main elements of cost for a control system are control hardware, consultant manpower cost and technology cost. The costs are mainly estimated from experience with similar projects and installation cost as quoted by a vendor.

9. Cost/benefit analysis is considered important at all stages of the implementation of a control system, with the analysis before a controller is implemented considered to be more important than the after-controller implementation and the follow-up analysis.

10. One-third of the respondents indicated the economic assessment of grinding control needed to be improved. 


\section{Chapter 6}

\section{Performance function development: Grinding mill circuits}

\subsection{Introduction}

As shown in Chapter 5, grinding mill circuits are very important unit processes in the mineral processing industry. The economic objectives of grinding circuits are to produce a product, at a reasonable throughput, from which good recovery can be achieved in the downstream processes, and to reduce operating cost. The objectives can usually be classified into two categories, i.e. increasing benefits and decreasing costs. The economic performance of milling control systems is often not satisfactorily estimated (Hulbert, 2002; Radhakrishnan, 1999). The auditing of grinding circuits' economic performance remains important (Wills and Napier-Munn, 2006). PFs of grinding mill circuits are developed in this chapter, which are also reported in Wei and Craig (2009b).

The operation of the milling circuits has a great influence on the economic performance of a mineral processing plant, but investigating the economics of grinding circuits on their own cannot provide a satisfactory solution, as the most important economic influence of the milling operation occurs in the downstream processes (Hodouin et al., 2001). A unifying study that investigates the grinding and flotation circuits jointly is therefore used to quantify the economic performance of the milling circuit.

The PFs of grinding mill circuits are explored in three ways, i.e. a survey study, a literature survey and a plant test. The understanding of grinding circuit economics using survey research is discussed in Chapter 5. A plant test in which some PF-related information is revealed is discussed in Section 6.2. Assumptions intended to facilitate the PF modelling are made in Section 6.3. PFs of a given grinding circuit are then developed in Section 6.4. In Section 6.5 it is shown how to formulate the grinding circuit control system's economic performance index, based on the PFs developed. Conclusions are drawn in Section 6.6. 


\subsection{Obtaining performance function-related information from a plant test}

For Witwatersrand gold-bearing ores, leaching is mostly used for concentration after grinding. The product of grinding mill circuits is thickened first and then dissolved into a cyanide solution. The solution and solids are then separated by filtration, followed by clarification of the solution to remove the solids further. The gold is recovered from the solution subsequently.

An investigation into the relation of residue (gold not recovered) vs milling circuit product particle size is done in Craig et al. (1992b). In this work, a ROM milling circuit with single stage classification is used. The economic impact of the improved product particle size control cannot be quantified directly from the milling circuit itself and therefore an investigation of the relation of residue vs particle size resulting from a leaching process was conducted.

A sample of product was taken from the circuit and split into thirty $500 \mathrm{~g}$ samples. Three samples were used for judging the head grade and three samples were roll-leached in bottles to determine the first point on the curve of residue vs particle size. The other 24 samples were milled in a laboratory mill in batches of three for a length of period in such a way that regularly spaced grind points could be gained; the samples were then leached and the residue determined. A curve of residue vs particle size ( $\mathrm{PF}$ of particle size) was then formed using the data points obtained, as shown in Fig. 6.1. This curve makes sense considering that the bigger the percentage particle size smaller than $75 \mu \mathrm{m}$, the better liberation and the more gold is extracted by the leaching process.

Furthermore, it is worth mentioning that in order to conduct this experiment, the grinding circuit needs to be first regulated to run in steady state with a coarse output $(56 \%<75 \mu \mathrm{m})$ and then regulated back again. This means the experiment adversely interrupted the normal running of the circuit, which is usually not allowed. In addition, the derivation of the regularly spaced points needs abundant experience of the participator. This experiment is therefore unlikely to be repeated.

Comment: In this case, the physical meaning of the PF is residue in the leaching process, which is the major economic impact of particle size. However, it may not hold in other grinding processes, as the focus may be different, e.g. being the recovery instead of residue. Even for the same type of processes, the primary economic impact can be different. Also flotation, as opposed to leaching, is a much more common means of concentration.

\subsection{Assumptions used for the performance function de- velopment}

Owing to the highly complex nature of the economic relations in most processes, assumptions usually need to be made in order to facilitate the development of PFs (Oosthuizen et al., 2004; Zhou and Forbes, 2003). The assumptions are:

1. The economic impact of the controlled variables is independent of each other. (See Wei 


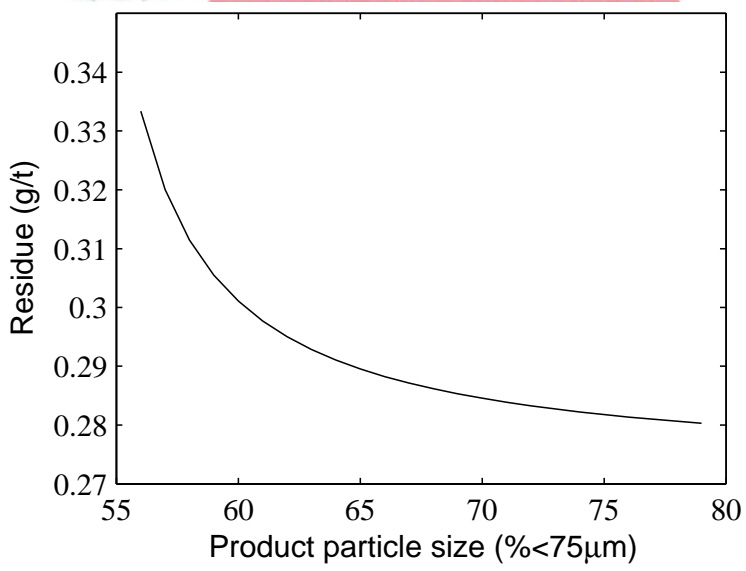

Figure 6.1: Economic impact of particle size (Craig et al., 1992b)

et al. (2007) for instances where this is not the case.)

2. When the grind fineness varies, the mineral recoveries will differ.

3. When the sump level (SLEV) or mill load (LOAD) violates predetermined limits, the grinding system will be shut down and then some compensative action will need to be taken in order to restore normal operation some time after the malfunction.

4. The product of the milling circuits is assumed to be the feed to the flotation circuit.

5. The head grade of the feed ore is assumed to be constant over the period of interest.

6. As far as the milling circuit product PSD vs flotation recovery relation is concerned, the operating cost of the flotation circuit is assumed to remain constant.

\subsection{Performance functions of a given grinding circuit}

In this section, the development of the PFs for a given milling circuit is discussed. For each CV of interest, the analysis of the information used to establish a PF is given firstly and then the $\mathrm{PF}$ derived is shown. The information required is obtained mainly from an in-depth literature study and a questionnaire survey (Wei and Craig, 2009c). Some results of the web-based questionnaire survey, as given in Chapter 5, are incorporated into the analysis below. Note that the PFs remain consistent regardless of control technologies used. They rather depend on the system dynamics, process economics, operational specifications and market situations.

\subsubsection{Process description and process variables}

The ROM ore milling circuit being considered for PF development is a closed one-stage circuit, comprising a semi-autogenous mill as shown in Fig. 6.2. It is assumed to process ore at approximately $100 \mathrm{t} / \mathrm{h}$ and to produce a product of which approximately $80 \%$ passes $75 \mu \mathrm{m}$. The mill discharges slurry into a sump via an end-discharge grate. The slurry is diluted in the sump by water and pumped to a hydrocyclone. Lighter particles pass to the cyclone overflow, being the milling circuit product, and heavier particles pass to the underflow of the cyclone. The 
underflow of the cyclone, water and feed ore comprise the mill feed (Stanley, 1987). The SLEV and LOAD are usually controlled, as they are open loop unstable. Considering the purpose of grinding mill circuits, the product particle size distribution (PSD) is also selected as a CV. In the following subsections, the PFs of these CVs are discussed.

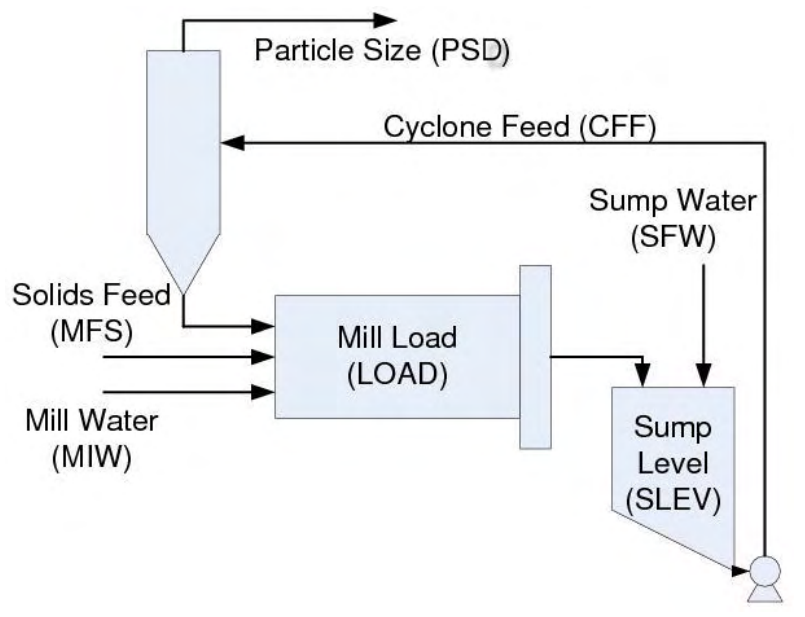

Figure 6.2: The structure of the milling circuit (adapted from Coetzee et al. (2008))

\subsubsection{Performance function for the particle size distribution}

The PF of a milling circuit product's PSD depends on the downstream concentration process. As flotation is used much more often than leaching, flotation will be used as concentration process in what follows. A literature survey was conducted on the steady-state relationship between the ore PSD of a grinding mill circuit's product and the mineral recovery in the downstream flotation circuit. This relation is also noted as the recovery vs grind relation for the sequel of this thesis. The survey reveals that the character of the recovery vs grind relation remains consistent for different minerals in various mineral processing plants, which is illustrated in Grant et al. (1991); Bascur (1991); Bazin et al. (1994a,b, 1995); Edwards and Vien (1999) and Sosa-Blanco et al. (1999), and will be reviewed next.

The consistency of the recovery vs grind relation arises from two consistencies, i.e. the consistency of the relation between the recovery and the mineral particle size (fraction/distribution) for feed to the flotation circuit and the consistency between the mineral PSD and ore PSD of the milling circuits product. Research into the relation between recovery and particle size (fraction/distribution) in flotation circuits has remained important in the mineral processing industry and can be classified into two stages. In the first stage, the relation between the recovery and particle size fraction is investigated. In the second stage, the relation between the recovery and the mineral particle size distribution is considered.

Recovery vs mineral particle size fraction The first in-depth study of the effect of particle size on flotation was conducted by Gaudin et al. (1931). The performance of a number of concentrators was analysed and it was found that the best recoveries of lead, zinc and copper were obtained for particles between 10 and $50 \mu \mathrm{m}$ diameter. The relevant recovery-size curves are asymmetric in shape. Recovery falls sharply above 100 micron but gradually below 10 micron.

A popular method to obtain the relation between the recovery and particle size fraction is to 
divide the feed to the flotation circuit into different size fractions, e.g. 10 to $20 \mu \mathrm{m}$ and 35 to $52 \mu \mathrm{m}$ and so on, then to record the average size of each fraction, to perform flotation on each fraction, and then to record the mineral recovery for each fraction. Consequently, for each fraction, a point associated with an average particle size and the relevant recovery is established. The overall relation between the recovery and particle size fraction can then be formed based on all these points.

Recovery vs size fraction curves are usually fairly stable in shape for a given set of flotation conditions (Trahar, 1981). The shape of the curve is regarded to be reasonably independent of moderate variations in particle size distribution, valuable mineral content and pulp density. The characteristics of the curves used to formulate the relations, in both real plants and laboratory batch tests, have been regarded as highly consistent in a number of studies (Trahar, 1981; Trahar and Warren, 1976; Lynch and Bush, 1977; Lynch, 1981; Jones et al., 1991; Bascur, 1991; Feng and Aldrich, 1999), as shown, e.g., in Fig. 6.3.

The ore particles can be roughly classified into three sections, namely those for fine particles, intermediate particles and coarse particles (Trahar, 1981). The ore may contain various minerals and not all minerals show maximum recovery in the same size ranges. The slower flotation of fine particles results from a lower rate of attachment of particles to bubbles. Fine particles have a lower rate of collision with bubbles and a lower momentum, which reduces the probability of adhesion and subsequent flotation (Trahar and Warren, 1976). Two main mechanisms can account for the behaviour of coarse particles. The first is that the degree of hydrophobicity needed to facilitate a high level of floatability increases with the increase in particle size. The second is that rapid collector consumption by the fine particles causes less surface coverage of the coarse particles, which consequently are less floatable.

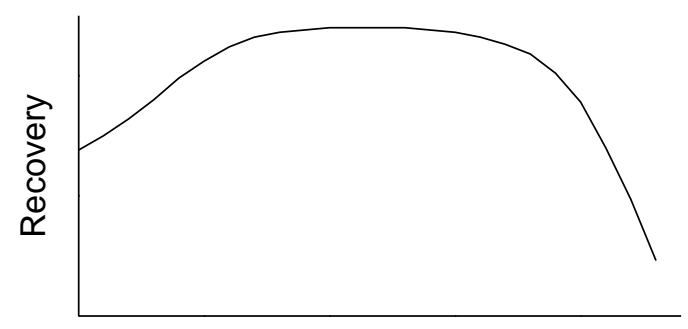

Particle size

Figure 6.3: Recovery vs size fraction relation (from Lynch and Bush (1977))

Recovery vs mineral particle size distribution In the second stage, the relationship between the recovery and the particle size distribution is considered. Note that in this context the Rosin-Rammler distribution is used to reflect the particle size distributions, describing the percentage of ore passing a given size.

The effect of the size distribution on the flotation recovery has also been investigated and it has been discovered that the types of relations also remain consistent for various minerals in both industrial flotation circuits and laboratory tests (Grant et al., 1991; Bazin et al., 1994b, 1995; Edwards and Vien, 1999; Sosa-Blanco et al., 1999). The recovery vs mineral PSD relation is derived from the recovery vs particle size fraction relation and a transformation from mineral PSD to the probabilities that the mineral falls into various size fractions. The results for the first stage and those for the second stage are inherently consistent owing to this transformation.

The primary hindrance to establishing this relation results from the difficulty in predicting the 
mineral PSD of the feed to flotation circuits from the ore PSD of the milling circuit product (King, 1979; Herbst et al., 1988; Sosa-Blanco et al., 1999). However, the relation between ore PSD and mineral PSD has also been found to be consistent, for a given mineral processing plant, as shown in King (1979); Grant et al. (1991); Bazin et al. (1994a, 1995) and Sosa-Blanco et al. (1999).

The consistency of the recovery vs grind for a given mineral processing plant is therefore established. A typical curve for the recovery vs grind relation is a quadratic function. This consistency has a significant influence on the economic performance assessment of milling circuits, since the economic impact of the downstream flotation circuit is included without additional examination of the flotation circuit. The relation can be used as a PF in the context of EPA of milling circuit controllers.

When the recovery vs milling circuit product's PSD relation is determined, it is relatively straightforward to calculate the value of the mineral recovered over a given period using Eq. (6.1):

$$
\begin{aligned}
\text { Mineral value }[\$]= & \text { (throughput rate }[t / h]) \times(\text { time period }[h]) \times(\text { head grade }[g / t]) \\
& \times(\text { recovery }[\%]) \times(\text { mineral price }[\$ / g])
\end{aligned}
$$

For the milling circuit being considered, the PF for the milling circuit product's PSD is derived as:

$$
\vartheta\left(y_{1}\right)=a y_{1}^{2}+b y_{1}+c
$$

where $a=-0.009776, b=1.705$ and $c=-2.955$. The PF is shown in Fig. 6.4. The maximum recovery is $71.4 \%$ and the relevant operating point is $87.2 \%$ passing $75 \mu \mathrm{m}$ (Edwards and Vien, 1999).

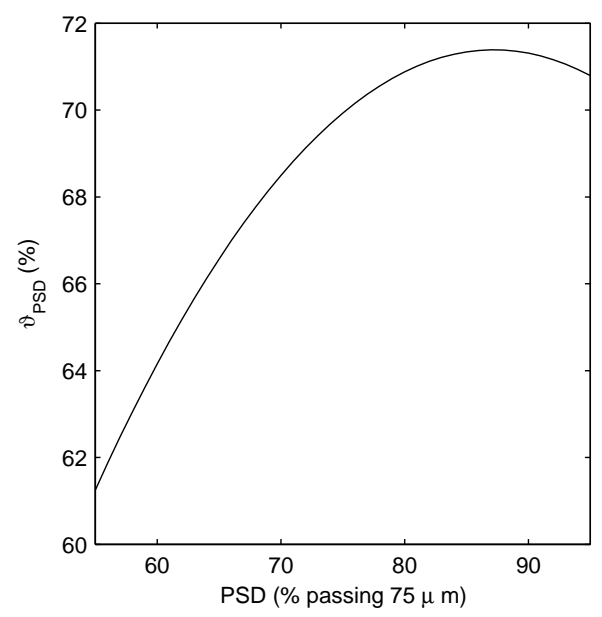

Figure 6.4: PF (recovery) of the ore PSD of the milling circuit product (adapted from Fig. 7 in Edwards and Vien (1999))

\subsubsection{Performance function for the mill load}

The LOAD is controlled within a range to ensure the stability of the system. When the LOAD is slightly higher or lower than the nominal value, some compensative action will be taken. 
Performing such action is routine for the controller and does not cause additional cost/benefit. When the mill load exceeds predetermined upper or lower limits, the operation needs to be stopped and compensative action will be conducted in order to restore routine operation (Wei and Craig, 2009c).

The PF of the LOAD can therefore be described as a piece-wise function consisting of three ranges (see Fig. 6.5). The middle range is the one within which the LOAD is expected to stay. The other two ranges relate to where the LOAD exceeds an upper/lower limit. In the middle range, the economic impact will be zero since the operation is within specification and there is no additional cost and profit. The PF of the LOAD is unsymmetrical, as the compensative actions for a too low LOAD and a too high LOAD are not necessarily the same and accordingly may cause different expenses. Note that the setpoint of the LOAD is often changed dynamically to maximise the power drawn from the mill motor, as optimum grinding is often regarded to take place when this happens in the middle range (Craig et al., 1992b). Such an optimiser will not be considered in this study. Because of the relatively simple dynamics of the LOAD, it can usually be controlled well and the possibility of violating the constraints is pretty small. So it is not the focus of this paper. The same argument applies to the SLEV as well.

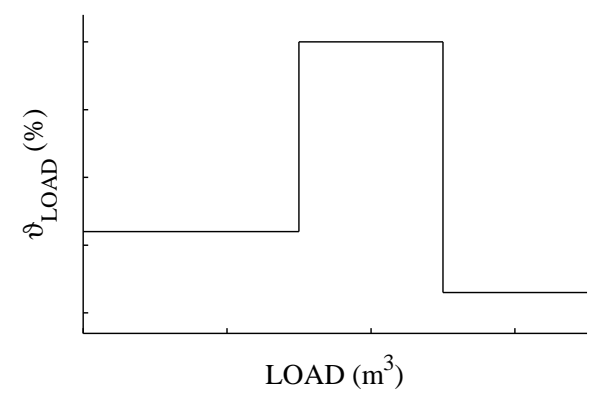

Figure 6.5: PF of the LOAD

\subsubsection{Performance function for the sump level}

The specification for the SLEV is also to stay within a selected range. When the SLEV exceeds a predetermined upper or lower limit, compensative action will be taken, which will have some economic consequences (Wei and Craig, 2009c).

The PF of the SLEV can also be described by a piece-wise function consisting of three ranges; see Fig. 6.6. The middle range is the one in which the SLEV is expected to stay. The other two ranges are those in which the SLEV exceeds the upper/lower limit. In the middle range, the economic impact will be zero since the operation is within specification and there is no additional cost and profit. The PF of the SLEV is unsymmetrical, as the compensative action for too low a SLEV and too high a SLEV are not necessarily the same and accordingly may cause different expenses. In this work the SLEV is controlled at a fixed setpoint for comparative purposes, but as the sump acts as a buffer, it is only required to be range-bound (Hulbert et al., 1990). 


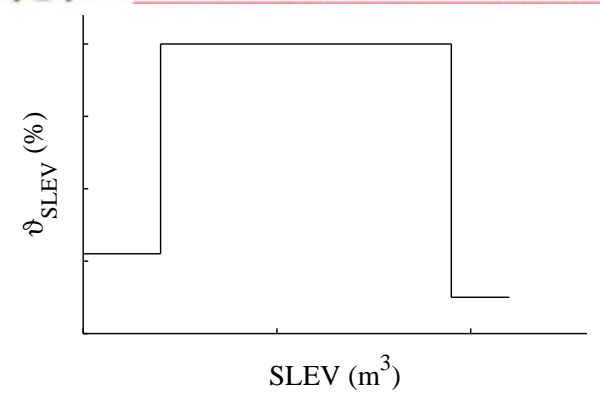

Figure 6.6: PF of the SLEV

\subsection{Overall economic performance of the circuit}

The model used to describe the overall economic performance of the milling circuit is discussed in this section. The notation used to indicate the overall EPI of the system is given in Table 6.1. The overall EPI of the circuit is given in Eq. (6.3).

$$
\Psi=\Psi_{y_{1}}+\Psi_{y_{2}}+\Psi_{y_{3}}
$$

where

$$
\Psi_{y_{i}}=\int_{-\infty}^{\infty} v\left(y_{i}\right) f\left(y_{i}\right) d y_{i}
$$

where for each considered CV

$$
\begin{aligned}
v\left(y_{i}\right) & =\Delta P\left(y_{i}\right)-\Delta C_{\left(y_{i}\right)} \\
& =\Delta P_{m}\left(y_{i}\right)+\Delta P_{f}\left(y_{i}\right)-\Delta C_{m}\left(y_{i}\right)-\Delta C_{f}\left(y_{i}\right)
\end{aligned}
$$

Here it is important to comprehend that the $\Delta P\left(y_{i}\right)-\Delta C_{\left(y_{i}\right)}$ is an abstract general form of $\mathrm{PF}$ and in a given process for a selected $\mathrm{CV}$ it may have a specific form, e.g., for the $\mathrm{PF}$ of the PSD of the milling circuit being considered, its form is $a y_{1}^{2}+b y_{1}+c$. The general form is consistent with the specific form.

Table 6.1: Notation of symbols used in expressing performance

\begin{tabular}{cl}
\hline Variable & Description \\
\hline$y_{1}$ & PSD \\
$y_{2}$ & LOAD \\
$y_{3}$ & SLEV \\
$\Psi$ & The overall EPI for a given period \\
$\Psi_{y_{i}}$ & The EPI with reference to $y_{i}$ \\
$v\left(y_{i}\right)$ & PF for $y_{i}$ \\
$f\left(y_{i}\right)$ & PDF of $y_{i}$ \\
$\Delta C\left(y_{i}\right)$ & Additional cost for $y_{i}$ compared to the base level \\
$\Delta C_{m}\left(y_{i}\right)$ & Additional milling circuit cost for $y_{i}$ compared to the base level \\
$\Delta C_{f}\left(y_{i}\right)$ & Additional flotation circuit cost for $y_{i}$ compared to the base level \\
$\Delta P\left(y_{i}\right)$ & Additional profit for $y_{i}$ compared to the base level \\
$\Delta P_{m}\left(y_{i}\right)$ & Additional milling circuit profit for $y_{i}$ compared to the base level \\
$\Delta P_{f}\left(y_{i}\right)$ & Additional flotation circuit profit for $y_{i}$ compared to the base level \\
\hline
\end{tabular}

Note that the integral, as described in Eq. (6.4), usually corresponds to the data collected over a period, either from a real plant or a simulation. The PDFs $\left(f\left(y_{i}\right)\right)$ can be derived from the operating data of the CVs. The PF for each CV $\left(v\left(y_{i}\right)\right)$ is described in Section 6.4. 


\subsection{Conclusion}

In this chapter, PFs of grinding mill circuits are examined based on the information resulting from survey research, a literature survey and a plant test.

Firstly, survey research is done to determine the current practice of control and functional/economic performance of grinding mill circuits in the mineral processing industry, as elaborated on in Chapter 5. The research is done using the distribution and collection of a web-based questionnaire and an MS Word document. In this research, information required to develop PFs of grinding circuits is requested and instructive results are obtained.

An in-depth literature survey undertaken to study the economics of grinding mill circuits reveals that the main economic impact of CVs for grinding circuits often occurs in the downstream process. Furthermore, the biggest impact is on mineral recovery in the flotation circuits. Then the steady-state relationship between the the grinding circuit product ore PSD and mineral recovery in the flotation circuit is examined in a literature study. Fruitful information is collected in this study, and the PF of the ore PSD of the grinding circuit is developed for the circuit, which will be used in the EPA of three controllers that will be discussed in Chapter 7 . Moreover, after selecting suitable performance measures and synthesising the results obtained from the survey research, the PFs of the LOAD and SLEV are also established for the focussed milling circuit.

When it is decided to use different performance measures, the physical meaning of the PFs and accordingly the resultant functions could be quite different. A plant test is reviewed in which the major economic impact of the controller of the milling circuit lies in the tailing grade of the downstream process. A PF is developed based on plant tests. It turns out that plant tests can help to develop PFs, but they are often infeasible in practice. 


\section{Chapter 7}

\section{Economic performance assessment of three milling circuit controllers}

\subsection{Introduction}

The objective of this chapter is to conduct the EPA of three single-loop PID controllers and an NMPC for the ROM milling circuit, as described in Section 6.4.1, using the framework elaborated on in Chapter 3. The intention of the EPA is to show the central role of PFs in the EPA and how to conduct an EPA systematically. The EPA is also intended to investigate the advantage of the NMPC controller with regard to the economic performance. Note that in this chapter the emphasis is on showing how to perform EPA systematically instead of identifying the best possible controller. The contents of this chapter are partially reported on in Wei and Craig (2009b). The PFs of the milling circuit developed a priori in Section 6.4, based on a web-based survey and an in-depth literature investigation, play a key role in this assessment.

The organisation of this chapter is as described below. The process model of the milling circuit considered is given in Section 7.2. The EPA of the circuit is conducted in Sections 7.3 to 7.7. The base case identification with single-loop PID controllers is discussed in Section 7.3 and the NMPC controller design is discussed in Section 7.4. The estimation of functional performance and initial economic assessment with NMPC is given in Section 7.5. The assumption for using the NMPC controller is made in Section 7.6. In Section 7.7, the functional performance of the NMPC controller is investigated using a statistical experiment in which the NMPC and singleloop PID controllers are switched according to a completely randomised designed experiment; then an economic assessment is conducted to illustrate the economic performance improvement resulting from the NMPC control compared to single-loop PID controllers. The assessment results are discussed in Section 7.8 and conclusions are drawn in Section 7.9. 


\subsection{Models and control objectives of the milling circuit}

For the milling circuit being considered, the PSD, LOAD and SLEV are selected for CVs, as shown in Section 6.4.1. The selected manipulated variables are the feed rate of solids to the mill (MFS), the feed rate of water to the sump (SFW) and the cyclone feed flow rate (CFF). These manipulated and controlled variables are used frequently for milling circuit control (Wei and Craig, 2009c).

\subsubsection{Nonlinear model of the milling circuit}

The time-domain nonlinear model used in Coetzee et al. $(2008,2009)$ is employed in this study. The relevant process variables and states are shown in Fig. 7.1. It comprises independent modules for the feeder, mill, sump and hydrocyclone. The separate modules can be linked to simulate various circuit configurations. The circuit has six inputs, five outputs and eight states. The model is given in this subsection for the purpose of clarification. The nomenclature used in the model is given in Table 7.1.

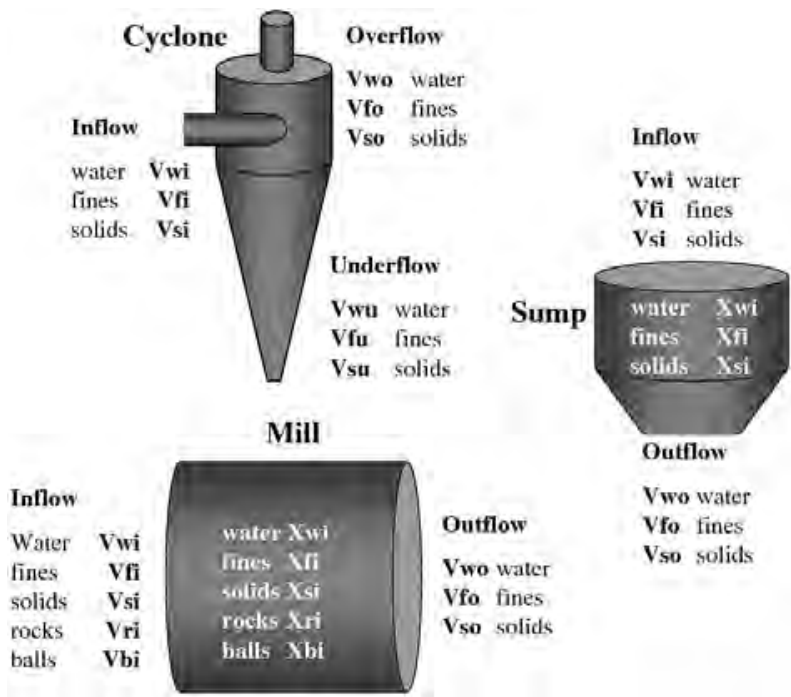

Figure 7.1: The modules of the circuit model (Coetzee et al., 2008)

\subsubsection{Feeder module}

The feeder takes the ore and divides it into streams. The feed ore comprises rock (that does not discharge from the mill), the coarse (out of specification but can discharge from the mill) and fines (in-specification material). Solids are defined as the sum of the fines and the coarse ore. The composition of the fine ore and rock is denoted by $\alpha_{f}$ and $\alpha_{r}$ respectively, as seen in 
Table 7.1: The milling circuit model's nomenclature

\begin{tabular}{|c|c|}
\hline Symbol & Description \\
\hline & Volumetric flow rates: in $(i)$, out $(o)$, underflow $(u)$ \\
\hline$V_{w i}, V_{w o}, V_{w o}$ & Water $\left[\mathrm{m}^{3} / \mathrm{h}\right]$ \\
\hline$V_{s i}, V_{s o}, V_{s o}$ & Solids $\left[\mathrm{m}^{3} / \mathrm{h}\right]$ \\
\hline$V_{c i}, V_{c o}, V_{c u}$ & Coarse $\left(\mathrm{V}_{s}-V_{f}\right)\left[\mathrm{m}^{3} / \mathrm{h}\right]$ \\
\hline$V_{f i}, V_{f o}, V_{f u}$ & Fines $\left[\mathrm{m}^{3} / \mathrm{h}\right]$ \\
\hline$V_{r i}, V_{\text {ro }}$ & $\operatorname{Rock}\left[\mathrm{m}^{3} / \mathrm{h}\right]$ \\
\hline$V_{b i}, V_{b o}$ & Ball $\left[\mathrm{m}^{3} / \mathrm{h}\right]$ \\
\hline$V_{g i}$ & Feed ore $\left(\mathrm{V}_{r i}+\mathrm{V}_{s i}\right)\left[\mathrm{m}^{3} / \mathrm{h}\right]$ \\
\hline$V_{t i}$ & Total feed flow-rate $\left(\mathrm{V}_{w i}+\mathrm{V}_{c i}+\mathrm{V}_{f i}\right)\left[\mathrm{m}^{3} / \mathrm{h}\right]$ \\
\hline & Volumetric-based states $\left[\mathrm{m}^{3} / \mathbf{h}\right]$ \\
\hline$X_{w}$ & Water $\left[\mathrm{m}^{3}\right]$ \\
\hline$X_{s}$ & Solids $\left(\mathrm{X}_{c}+\mathrm{X}_{f}\right)\left[\mathrm{m}^{3}\right]$ \\
\hline$X_{f}$ & Fines $\left[\mathrm{m}^{3}\right]$ \\
\hline$X_{r}$ & Rocks $\left[\mathrm{m}^{3}\right]$ \\
\hline$X_{b}$ & Balls $\left[\mathrm{m}^{3}\right]$ \\
\hline$X_{h}$ & Total load $\left(\mathrm{X}_{w}+\mathrm{X}_{s}+\mathrm{X}_{r}\right)\left[\mathrm{m}^{3}\right]$ \\
\hline & Mill manipulated variables \\
\hline$\alpha_{\text {speed }}$ & Mill speed fraction \\
\hline & Sump manipulated variables \\
\hline $\mathrm{V}_{\text {water-add }}$ & Volumetric flow-rate of water to the sump $\left[\mathrm{m}^{3} / \mathrm{h}\right]$ \\
\hline$V_{t o}$ & Total flow-rate from sump (Determined by the pump speed) $\left[\mathrm{m}^{3} / \mathrm{h}\right]$ \\
\hline & Feeder parameters \\
\hline$\alpha_{f}$ & Fraction of fines in the ore \\
\hline$\alpha_{r}$ & Fraction of rock in the ore \\
\hline & Mill parameters \\
\hline$\eta_{f}$ & Power per fines produced $[\mathrm{kWh} / \mathrm{t}]$ \\
\hline$\varepsilon_{w s}$ & Maximum water-to-solids volumetric ration at zero pulp flow \\
\hline$\phi_{r}$ & Rock abrasion factor $[\mathrm{kWh} / \mathrm{t}]$ \\
\hline$\phi_{b}$ & Steel abrasion factor $[\mathrm{kWh} / \mathrm{t}]$ \\
\hline$V_{V}$ & Volumetric flow per 'flowing volume' driving force $\left[\mathrm{h}^{-1}\right]$ \\
\hline$P_{\max }$ & Maximum power $[\mathrm{kW}]$ \\
\hline$\delta_{P v}$ & Power-change parameter for volume \\
\hline$v_{P \max }$ & Mill filling volume for maximum power $\left[\mathrm{m}^{3}\right]$ \\
\hline$\delta_{P s}$ & Power-change parameter for fraction solids \\
\hline$\varphi_{P \max }$ & Rheology factor for maximum power \\
\hline & $\begin{array}{l}\text { Fractional power reduction per fractional reduction from maximum mill } \\
\text { speed }\end{array}$ \\
\hline$v_{\text {mill }}$ & Mill volume $\left[\mathrm{m}^{3}\right]$ \\
\hline$\alpha_{\eta_{f}}$ & $\begin{array}{l}\text { Fractional change in } \mathrm{kW} / \text { fines produced per change in fractional filling of } \\
\text { mill }\end{array}$ \\
\hline$\chi_{P}$ & Cross-term for maximum power \\
\hline$D_{s}$ & Density of ore $\left[\mathrm{kg} / \mathrm{m}^{3}\right]$ \\
\hline$D_{b}$ & Density of steel balls $\left[\mathrm{kg} / \mathrm{m}^{3}\right]$ \\
\hline & Cyclone parameters \\
\hline$F_{i}$ & Fraction of total feed consisting of solids \\
\hline$P_{i}$ & Fraction of total feed consisting of fines \\
\hline$F_{u}$ & Fraction of total underflow consisting of solids \\
\hline$\varepsilon_{c}$ & Coarse split \\
\hline$\alpha_{s u}$ & Fraction solids in underflow \\
\hline$C_{1}, C_{3}, C_{4}, C_{5}$ & Constants \\
\hline
\end{tabular}


Table 7.1. The dynamics of the feeder is described by the following equations

$$
\begin{aligned}
& V_{w o} \triangleq V_{w i} \\
& V_{s o} \triangleq V_{g i}\left(1-\alpha_{r}\right) \\
& V_{f o} \triangleq \alpha_{f} \cdot V_{g i} \\
& V_{r o} \triangleq \alpha_{r} \cdot V_{g i} \\
& V_{b o} \triangleq V_{b i}
\end{aligned}
$$

\subsubsection{Mill module}

The feed of the mill comprises three flow rates: the feed rate of fines $\left(V_{f i}\right)$, of solids $\left(V_{s i}\right)$ and of rocks $\left(V_{r i}\right)$ in $\mathrm{m}^{3} / \mathrm{h}$. There are two additional feeds: the flow rate of water to the mill $\left(V_{w i}\right)$ and the feed rate of balls to the mill $\left(V_{b i}\right)$. The mill has five states: the volume of water $\left(X_{m w}\right)$, solids $\left(X_{m s}\right)$, fines $\left(X_{m f}\right)$, rock $\left(X_{m r}\right)$ and balls $\left(X_{m b}\right)$ measured in $\mathrm{m}^{3}$. The LOAD is the summation of all the states of the mill. The mill has 14 parameters and the parameters characterising the ore hardness $\left(\eta_{f}, \phi_{r}, \phi_{b}\right)$ are shown in Table 7.1. The mill model also involves the impact of mill power and slurry rheology to the breakage and power functions. The changes in the holdups of the materials inside the mill are described by

$$
\begin{aligned}
& \frac{\partial X_{w}}{d t} \triangleq V_{w i}-V_{w o} \\
& \frac{\partial X_{s}}{\partial t} \triangleq V_{s i}-V_{s o}+R C \\
& \frac{\partial X_{f}}{\partial t} \triangleq V_{f i}-V_{f o}+F P \\
& \frac{\partial X_{r}}{\partial t} \triangleq V_{r i}-R C \\
& \frac{\partial X_{b}}{\partial t} \triangleq V_{b i}-B C
\end{aligned}
$$

for which the discharge flow rates of the materials inside the mill are defined by

$$
\begin{aligned}
& V_{t o} \triangleq V_{V} \cdot \varphi \cdot X_{w} \\
& V_{w o} \triangleq V_{t o}\left(\frac{X_{w}}{X_{s}+X_{w}}\right) \\
& V_{s o} \triangleq V_{t o}\left(\frac{X_{s}}{X_{s}+X_{w}}\right) \\
& V_{f o} \triangleq V_{t o}\left(\frac{X_{f}}{X_{s}+X_{w}}\right) \\
& V_{r_{o}} \triangleq 0 \\
& V_{b o} \triangleq 0
\end{aligned}
$$

The production of fines (FP) is a linear function of the total load inside the mill and defined by

$$
F P \triangleq \frac{P_{\text {mill }}}{D_{s} \cdot\left\{\eta_{f} \cdot\left[1+\alpha_{\eta_{f}} \cdot\left(\frac{X_{h}}{v_{\text {mill }}}-v_{P \max }\right)\right]\right\}}
$$


Rock consumption (RC) is defined by

$$
R C \triangleq\left(\frac{1}{D_{s} \cdot \phi_{r}}\right) \cdot P_{m i l l} \cdot \varphi \cdot\left(\frac{X_{r}}{X_{r}+X_{s}}\right)
$$

Ball consumption (BC) is defined by

$$
B C \triangleq\left(\frac{1}{D_{b} \cdot \phi_{b}}\right) \cdot P_{\text {mill }} \cdot \varphi \cdot\left(\frac{X_{b}}{X_{b}+X_{r}+X_{s}}\right)
$$

The rheology factor is defined by

$$
\varphi \triangleq\left\{\frac{\max \left[0,\left(X_{w}-\left(\left(\frac{1}{\varepsilon_{w s}}\right)-1\right) X_{s}\right)\right]}{X_{w}}\right\}^{0.5}
$$

The mill power is defined by

$$
P_{\text {mill }} \triangleq P_{\text {max }} \cdot\left\{1-\delta_{P v} Z_{x}^{2}-2 \cdot \chi_{P} \cdot \delta_{P v} \cdot \delta_{P s} \cdot Z_{x} \cdot Z_{r}-\delta_{P s} \cdot Z_{r}^{2}\right\} \cdot\left(\alpha_{\text {speed }}\right)^{\alpha_{P}}
$$

where the impact of the load on the power consumption is defined by

$$
Z_{x} \triangleq \frac{X_{b}+X_{r}+X_{s}+X_{w}}{v_{P \max } \cdot v_{\text {mill }}-1}
$$

and the impact of rheology on power consumption is given by

$$
Z_{r} \triangleq \frac{\varphi}{\varphi_{P \max }}-1
$$

From the above equations, it can be seen that the mill is a highly nonlinear component.

\subsubsection{Sump module}

The sump feed consists of the mill discharge, including the flow rates of water $\left(V_{w i}\right)$, solids $\left(V_{s i}\right)$ and fines $\left(V_{f i}\right)$ given in $\mathrm{m}^{3} / \mathrm{h}$. The rock and balls are assumed to remain in the mill. The sump receives additional water $\left(V_{w i-s u m p}\right)$, i.e. SFW, to dilute the slurry in the sump. The sump discharge includes water $\left(V_{w o}\right)$, solids $\left(V_{s o}\right)$ and fines $\left(V_{f o}\right)$. The sump model has three states: the volume of water $\left(X_{s w}\right)$, solids $\left(X_{s s}\right)$ and fines $\left(X_{s f}\right)$ measured in $\mathrm{m}^{3}$. The SLEV is the summation of all the states of the sump. The changes of the holdups of materials inside the sump are defined by

$$
\begin{aligned}
\frac{\partial X_{w}}{\partial t} & \triangleq V_{w i}+V_{w a t e r-a d d}-V_{w o} \\
\frac{\partial X_{s}}{\partial t} & \triangleq V_{s i}-V_{s o} \\
\frac{\partial X_{f}}{\partial t} & \triangleq V_{f i}-V_{f o}
\end{aligned}
$$

where the discharge flow rates of the constituents of the slurry inside the sump are defined by

$$
\begin{gathered}
V_{w o} \triangleq V_{t o} \cdot\left(\frac{X_{w}}{X_{s}+X_{w}}\right) \\
V_{s o} \triangleq V_{t o} \cdot\left(\frac{X_{s}}{X_{s}+X_{w}}\right) \\
V_{f o} \triangleq V_{t o} \cdot\left(\frac{X_{f}}{X_{s}+X_{w}}\right)
\end{gathered}
$$


The sump level is defined by

$$
\mathrm{SLEV} \triangleq X_{w}+X_{s}
$$

The cyclone feed density is defined by

$$
\mathrm{CFD} \triangleq X_{w}+D_{s} \cdot X_{s} / X_{w}+X_{s}
$$

This module is also nonlinear.

\subsubsection{Hydrocyclone module}

The hydrocyclone feed consists of water $\left(V_{w i}\right)$, solids $\left(V_{s i}\right)$ and fines $\left(V_{f i}\right)$ received from the sump. This feed is separated into an overflow and underflow stream with the product reporting to the overflow $\left(V_{w o}+V_{s o}+V_{f o}\right)$ and the out-of-specification material $\left(V_{w u}+V_{s u}+V_{f u}\right)$ reporting to the underflow. The split is not perfect and both the overflow and underflow contain unwanted material. The cyclone has six parameters overall. The flow rates of the diverse materials at the underflow of the cyclone are defined by

$$
\begin{aligned}
V_{c u} & \triangleq V_{c i} \cdot\left(1-C_{1} \cdot e^{\left(-\frac{V_{t i}}{\varepsilon_{c}}\right)}\right) \cdot\left(1-\left(\frac{F_{i}}{C_{3}}\right)^{C_{4}}\right) \cdot\left(1-P_{i}^{C_{5}}\right) \\
F_{u} & \triangleq 0.6-\left(0.6-F_{i}\right) \cdot e^{\left(-\frac{V_{c u}}{\alpha_{s u} \varepsilon_{c}}\right)} \\
V_{w u} & \triangleq V_{w i} \cdot \frac{\left(V_{c u}-F_{u} \cdot V_{c u}\right)}{\left(F_{u} \cdot V_{w i}+F_{u} \cdot V_{f i}-V_{f i}\right)} \\
V_{f u} & \triangleq V_{f i} \cdot \frac{\left(V_{c u}-F_{u} \cdot V_{c u}\right)}{\left(F_{u} \cdot V_{w i}+F_{u} \cdot V_{f i}-V_{f i}\right)} \\
F_{i} & \triangleq \frac{V_{s i}}{V_{w i}+V_{s i}} \\
P_{i} & \triangleq \frac{V_{f i}}{V_{s i}}
\end{aligned}
$$

The product particle size is formulated as

$$
\mathrm{PSD} \triangleq \frac{V_{f i}-V_{f u}}{V_{c i}-V_{c u}+V_{f i}-V_{f u}}
$$

The product throughput is formulated as

$$
\text { THROUGHPUT } \triangleq V_{c o}+V_{f o}
$$

The cyclone is modelled by an efficiency curve that resembles an S-curve. The cyclone model is a nonlinear static model.

\subsubsection{Operating point and constraints}

In order to make the simulation of the process and control system as realistic as possible, a suitable operating point and process variable and parameter constraints are defined. The operating point and all the constraints on process states are given in Table 7.2. The operating point 
and constraints of the MVs and CVs are given in Table 7.3. Uncertainties in the parameters of interest are shown in Table 7.4. The ore hardness and rock fraction will be considered as primary uncertainties in the simulation studies conducted afterwards. The operating point will be different for various simulations. When only stationary centred disturbances are introduced, the operating point will be 28 and 0.1 respectively. When persistent disturbances are also introduced, the increase/decrease of average values will be added to the nominal values.

Table 7.2: Operating point and constraints (OP means operating point) of the process states

$\begin{array}{lllll}\text { Variable } & \text { Min } & \text { Max } & \text { OP } & \text { Description } \\ X_{m w} & 0 & 50 & 8.53 & \text { The holdup of water in the mill }\left[\mathrm{m}^{3}\right] \\ X_{m s} & 0 & 50 & 9.47 & \text { The holdup of ore in the mill }\left[\mathrm{m}^{3}\right] \\ X_{m f} & 0 & 50 & 3.54 & \text { The holdup of fine ore in the mill }\left[\mathrm{m}^{3}\right] \\ X_{m r} & 0 & 50 & 20.25 & \text { The holdup of rock in the mill }\left[\mathrm{m}^{3}\right] \\ X_{m b} & 0 & 20 & 6.75 & \text { The holdup of balls in the mill }\left[\mathrm{m}^{3}\right] \\ X_{s w} & 0 & 10 & 3.95 & \text { The holdup of water in the mill }\left[\mathrm{m}^{3}\right] \\ X_{s s} & 0 & 10 & 1.05 & \text { The holdup of ore in the sump }\left[\mathrm{m}^{3}\right] \\ X_{s f} & 0 & 10 & 0.14 & \text { The holdup of fine ore in the sump }\left[\mathrm{m}^{3}\right] \\ \text { MIW } & 0 & 100 & 33.33 & \text { The flow rate of water to the mill }\left[\mathrm{m}^{3} / \mathrm{h}\right] \\ V_{b i} & 0 & 4 & 2 & \text { The flow rate of balls to the mill }[\mathrm{t} / \mathrm{h}] \\ \alpha_{\text {millspeed }} & 0.7 & 1.0 & 0.82 & \text { The fraction of critical mill speed [dimensionless] } \\ \text { Rheology } & 0 & 1 & 0.51 & \text { The fluidity of the slurry in the mill [dimensionless] }\end{array}$

Table 7.3: Operating point and constraints (OP means operating point) of the MVs and CVs

\begin{tabular}{lllll} 
Variable & Min & Max & OP & Description \\
\hline MFS & 0 & 200 & 100 & The flow rate of ore to the mill $[\mathrm{t} / \mathrm{h}]$ \\
CFF & 400 & 500 & 443 & The flow rate of slurry from the sump to the cyclone $\left[\mathrm{m}^{3} / \mathrm{h}\right]$ \\
SFW & 0 & 400 & 267 & The flow rate of extra water to the sump $\left[\mathrm{m}^{3} / \mathrm{h}\right]$ \\
LOAD & 30 & 50 & 45 & The total charge of the mill $\left[\mathrm{m}^{3}\right]$ \\
SLEV & 2 & 9.5 & 5 & The level of the sump $\left[\mathrm{m}^{3}\right]$ \\
PSD & 50 & 90 & 80 & The particle size distribution of the circuit's product $[\%]$ \\
\hline
\end{tabular}

\subsubsection{Linearised model and input-output pairing selection of the milling circuit}

\subsubsection{Linearised model of the circuit}

A linear model is often obtained by conducting a first-order Taylor series expansion approximation for the relevant nonlinear model at a given equilibrium point (Skogestad and Postlethwaite, 2005). The modelling error can then be investigated by examining the discarded higher order terms. However, this is not the case in this work, as the linear model used in the controller design in this chapter is obtained in a different way.

Step tests and system identification are performed on the nonlinear model for all combinations of MVs and CVs. Multiple steps are used for each MV and different step sizes (amplitudes following designated uniform distribution are given randomly by Matlab) are used at each sampling time. Because of the multiple steps, the system moves around the operating point. Because the system is nonlinear, it will lead to different models being obtained. The step sequences are generated randomly, therefore leading to different results. Each step will give a slightly different response for the nonlinear model. An average model is obtained from the 
Table 7.4: Parameter values for the milling circuit ( $\Delta$ means the percentage value of the difference between the biggest uncertainty and the nominal value divided by the nominal value)

\begin{tabular}{|c|c|c|c|c|c|}
\hline Parame & r Nominal & Min & Max & $\% \Delta$ & Description \\
\hline$\alpha_{f}$ & 0.1 & 0.05 & 0.15 & 50 & Fraction of fines in the ore dimensionless] \\
\hline$\alpha_{r}$ & 0.1 & 0.02 & 0.18 & 80 & Fraction of rock in the ore [dimensionless] \\
\hline$\eta_{f}$ & 28 & 5.6 & 50.4 & 80 & Power per ton of fines produced $[\mathrm{kWh} / \mathrm{t}]$ \\
\hline$\varepsilon_{w s}$ & 0.6 & - & - & - & $\begin{array}{l}\text { Maximum water-to-solids volumetric ratio at zero } \\
\text { pulp flow [dimensionless] }\end{array}$ \\
\hline$\phi_{r}$ & 69 & 55 & 83 & 20 & Rock abrasion factor $[\mathrm{kWh} / \mathrm{t}]$ \\
\hline$\phi_{b}$ & 94 & 89 & 99 & 5 & Steel abrasion factor $[\mathrm{kWh} / \mathrm{t}]$ \\
\hline$V_{V}$ & 40 & - & - & - & $\begin{array}{l}\text { Volumetric flow per 'flowing volume' driving force } \\
{\left[\mathrm{h}^{-1}\right]}\end{array}$ \\
\hline$P_{\max }$ & 2000 & - & - & - & Maximum power $[\mathrm{kW}]$ \\
\hline$\delta_{P_{v}}$ & 1 & - & - & - & Power-change parameter for volume [dimensionless] \\
\hline$v_{P \max }$ & 0.45 & - & - & - & Mill filling volume for maximum power $\left[\mathrm{m}^{3}\right]$ \\
\hline$\delta_{P s}$ & 1 & - & - & - & $\begin{array}{l}\text { Power-change parameter for fraction solids [dimen- } \\
\text { sionless] }\end{array}$ \\
\hline$\varphi_{P \max }$ & 0.51 & - & - & - & Rheology factor for maximum power [dimensionless] \\
\hline$\alpha_{P}$ & 0.82 & - & - & - & $\begin{array}{l}\text { Fractional power reduction per fractional reduction } \\
\text { from maximum mill speed [dimensionless] }\end{array}$ \\
\hline$v_{\text {mill }}$ & 100 & - & - & - & Mill volume $\left[\mathrm{m}^{3}\right]$ \\
\hline$\alpha_{\eta_{f}}$ & 0.01 & - & - & - & $\begin{array}{l}\text { Fractional change in } \mathrm{kW} / \text { fines produced per change } \\
\text { in fractional filling of mill [dimensionless] }\end{array}$ \\
\hline$\chi_{P}$ & & - & - & - & Cross-term for maximum power [dimensionless] \\
\hline$\varepsilon_{c}$ & 184 & 175 & 193 & 5 & Coarse split [dimensionless] \\
\hline$\alpha_{s u}$ & 0.16 & 0.15 & 0.17 & 5 & Fraction solids in underflow [dimensionless] \\
\hline$C_{1}$ & 0.6 & - & - & - & Constant [dimensionless] \\
\hline$C_{3}$ & 0.7 & - & - & - & Constant dimensionless] \\
\hline$C_{4}$ & 3 & - & - & - & Constant [dimensionless] \\
\hline$C_{5}$ & 3 & - & - & - & Constant [dimensionless] \\
\hline
\end{tabular}


multiple step responses. The Matlab System Identification Toolbox is used for all the MV-CV combinations and the resultant transfer function matrix regarding the MVs and CVs of the models $(\hat{G})$ is

$$
\left[\begin{array}{c}
\operatorname{PSD}(\mathrm{s}) \\
\mathrm{SLEV}(\mathrm{s}) \\
\operatorname{LOAD}(\mathrm{s})
\end{array}\right]=\left[\begin{array}{ccc}
-0.00035 \frac{(1-0.63 s)}{(1+0.54 s)} e^{(-0.011 s)} & \frac{0.00055}{(1+0.24 s)} e^{(-0.011 s)} & \frac{-0.0043}{(1+0.58 s)} e^{(-0.065 s)} \\
\frac{-0.29}{s} & \frac{0.42}{s} & 0 \\
0.0028 \frac{1+0.876 s}{(1+3.868 s)} e^{(-0.0115 s)} & 0 & \frac{0.01}{s}
\end{array}\right]\left[\begin{array}{c}
\mathrm{CFF}(\mathrm{s}) \\
\mathrm{SFW}(\mathrm{s}) \\
\mathrm{MFS}(\mathrm{s})
\end{array}\right]
$$

The same procedure is used to reveal the relation between disturbances (Ore hardness $\left(\eta_{f}\right)$ and rock fraction $\left.\left(\alpha_{r}\right)\right)$ and CVs. The transfer function matrix is

$$
\left[\begin{array}{c}
\operatorname{PSD}(\mathrm{s}) \\
\operatorname{SLEV}(\mathrm{s}) \\
\operatorname{LOAD}(\mathrm{s})
\end{array}\right]=\left[\begin{array}{cc}
\frac{-0.24}{(1+0.54 s)} e^{(-0.014 s)} & 0.06 \frac{(1-0.001 s)}{(1+4.24 s)} e^{(-0.014 s)} \\
\frac{1.86 \times 10^{-3}}{(1+14.9 s)} e^{(-0.438 s)} & \frac{\left(1.75 \times 10^{-3}\right)}{\left(1+2(4.5)(14) s+(14 s)^{2}\right)} e^{(-0.014 s)} \\
\frac{0.58}{(1+1.41 s)} e^{(-0.089 s)} & \frac{-0.006}{\left(1+2(2.90)(6.25) s+(6.25 s)^{2}\right)(1+33.4 s)} e^{(-0.014 s)}
\end{array}\right]\left[\begin{array}{c}
\eta_{f}(s) \\
\alpha_{r}(s)
\end{array}\right]
$$

\subsubsection{Comparison between the linearised and nonlinear model}

In order to investigate the modelling error between the linear 'average' model and nonlinear model, multiple steps for each MV are done for both models and the responses of all three CVs are compared. When the CFF is considered, the resultant CVs are given in Fig. 7.2. When the SFW is considered, the resultant CVs are given in Fig. 7.3. When the MFS is considered, the resultant CVs are given in Fig. 7.4. From these three figures, it can be seen that the linear models regarding the PSD vs CFF and the PSD vs MFS are satisfactory approximation of the nonlinear model. The linear models with respect to the SLEV vs CFF, the LOAD vs CFF and the PSD vs SFW are acceptable approximations of the nonlinear model, despite some discrepancy. However, the linear models regarding the SLEV vs SFW and the LOAD vs MFS (too high linear gain) are disappointing approximations, although still suitable for control.

\subsubsection{Input-output pairing selection for the circuit}

The input-output pairings for single-loop controllers are essential, as the output should be paired with the input that has the greatest impact on the output and the input is supposed to have as little interaction as possible with other outputs. The conventional pairings for milling circuits are LOAD-MFS, PSD-SFW and SLEV-CFF (Wills and Napier-Munn, 2006). However, these pairings are problematic in practice, specifically when changes in ore hardness, feed rate, etc., take place (Chen et al., 2007) as explained in Section 7.2.3.3.1. This issue is confirmed by the preliminary simulations using the above mentioned pairings.

An alternative option, pairing LOAD-MFS, PSD-CFF and SLEV-SFW is adopted in this work, which is used in Smith et al. (2001) and Lynch (1979). The pairings turn out to have better robustness to the feed disturbances subject to actuator limitations than the traditional ones (Coetzee, 2009), as explained below. 


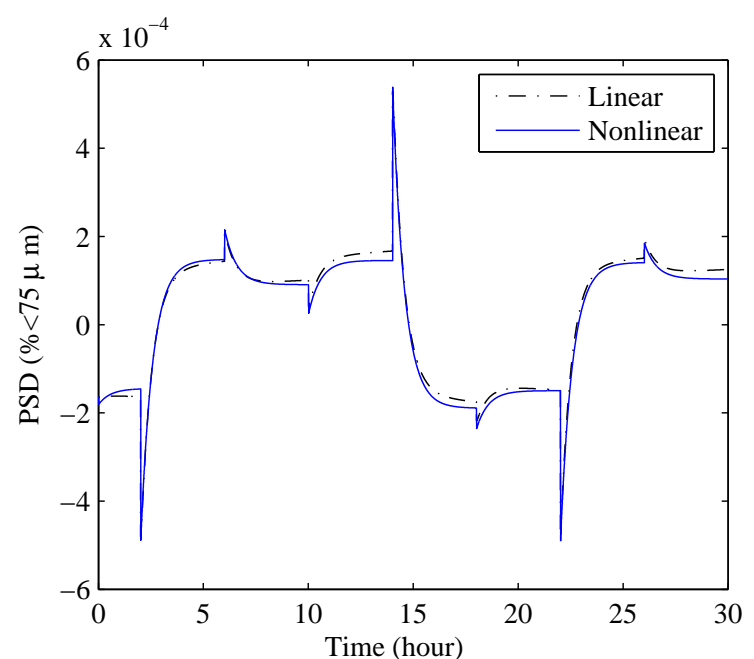

(a) Comparison for the PSD

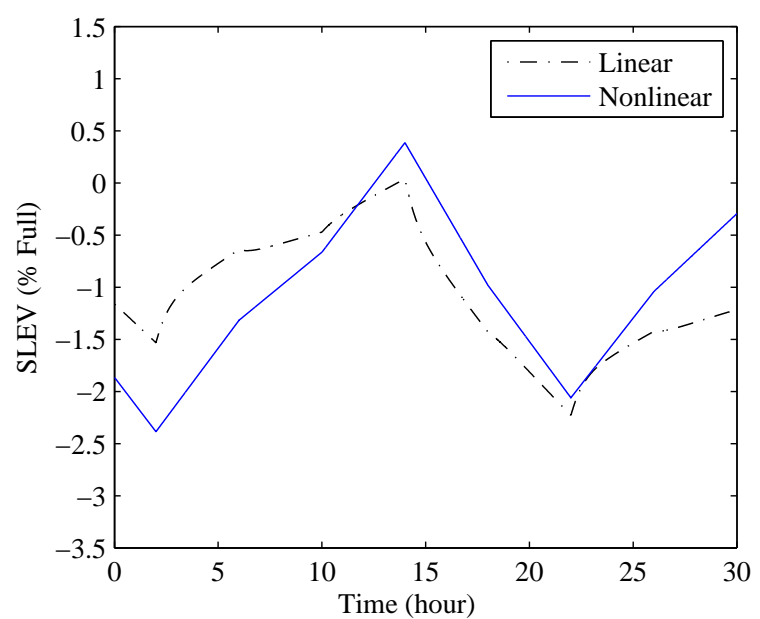

(b) Comparison for the SLEV

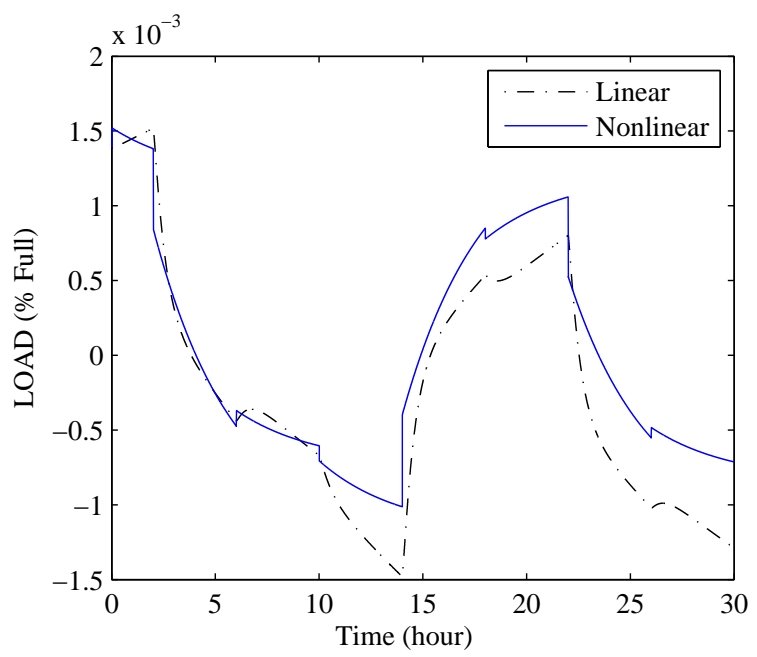

(c) Comparison for the LOAD

Figure 7.2: CVs responses to the multiple step tests of the CFF

\subsection{Further explanation of using the pairings PSD-CFF and SLEV-SFW:} When traditional pairings are used, if persistent disturbances are introduced into the circuit (which are important in the control problem in this chapter), e.g., the ore becomes harder, the process will easily become unstable. The stability results from the SLEV violating its upper and lower limits. The reason is that the SFW limits need to change due to the reduced production of the mill to maintain the SLEV within bounds if the SLEV-CFF and PSD-SFW parings are used. If the SFW is allowed to vary over its full range to try to achieve the desired PSD, it affects the SLEV more than what the CFF can compensate for within its limits.

The instability of the process happens due to the constraints on the SFW and CFF. The SFW has a bigger range of influence on SLEV: It can vary between $0-400 \mathrm{~m}^{3} /$ hour whereas the CFF can only change between $400-500 \mathrm{~m}^{3}$ /hour. The following two scenarios of SISO control are used to illustrate the instability.

Scenario 1: Assume that the mill is discharging at $170 \mathrm{~m}^{3} /$ hour of slurry, the process is at the nominal operating point with the SFW at $273 \mathrm{~m}^{3} /$ hour and the CFF at $443 \mathrm{~m}^{3} /$ hour. If the $\mathrm{PSD}$ setpoint requires the $\mathrm{CFF}$ to reach its $\max$ of $500 \mathrm{~m}^{3} /$ hour, then the $\mathrm{SFW}$ can increase 


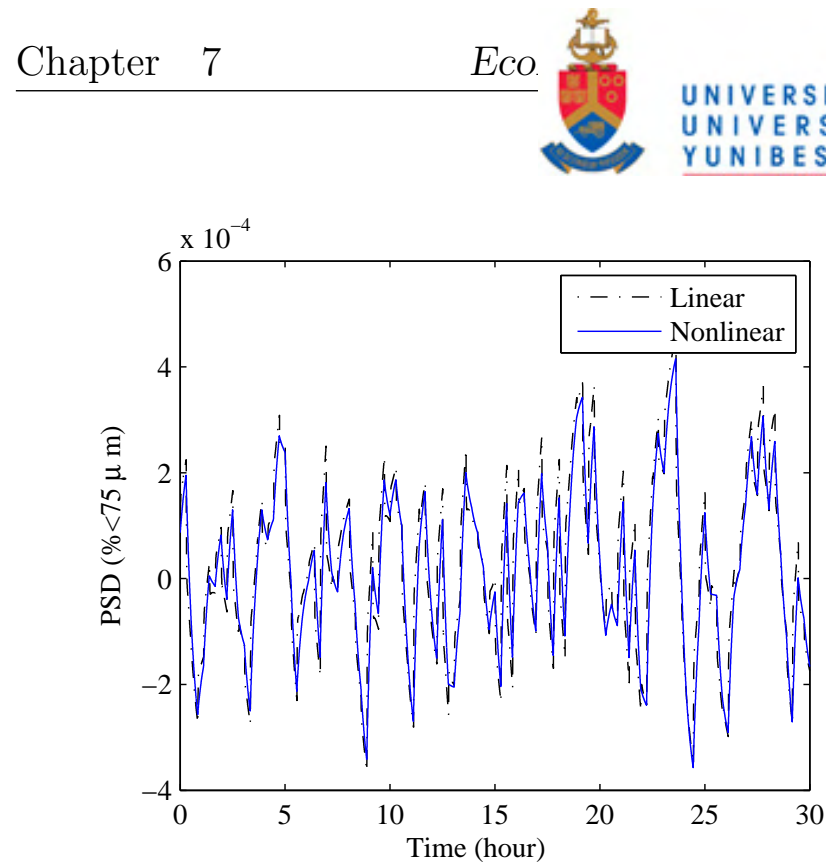

(a) Comparison for the PSD

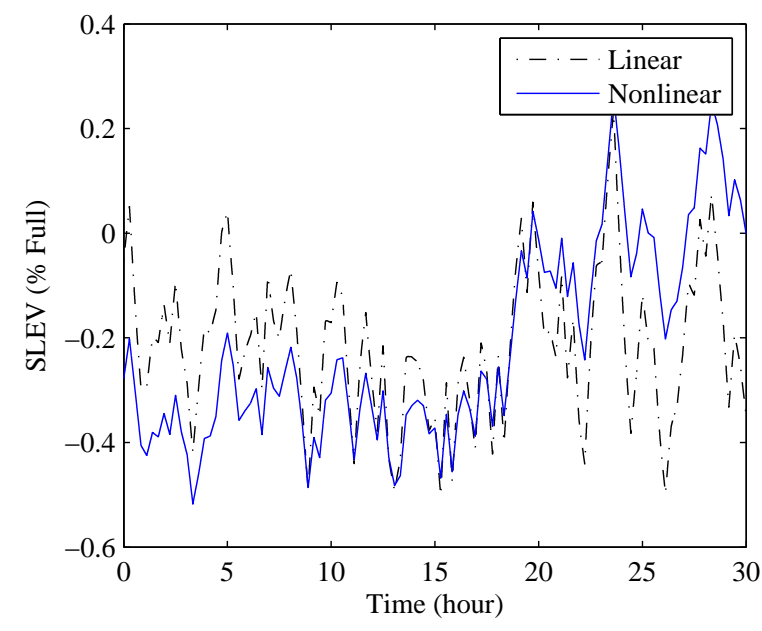

(b) Comparison for the SLEV

Figure 7.3: CVs responses to the multiple step tests of the SFW. No linear relation between the LOAD and SFW is revealed from the system identification based on the relevant step tests and accordingly no comparison is done for them.

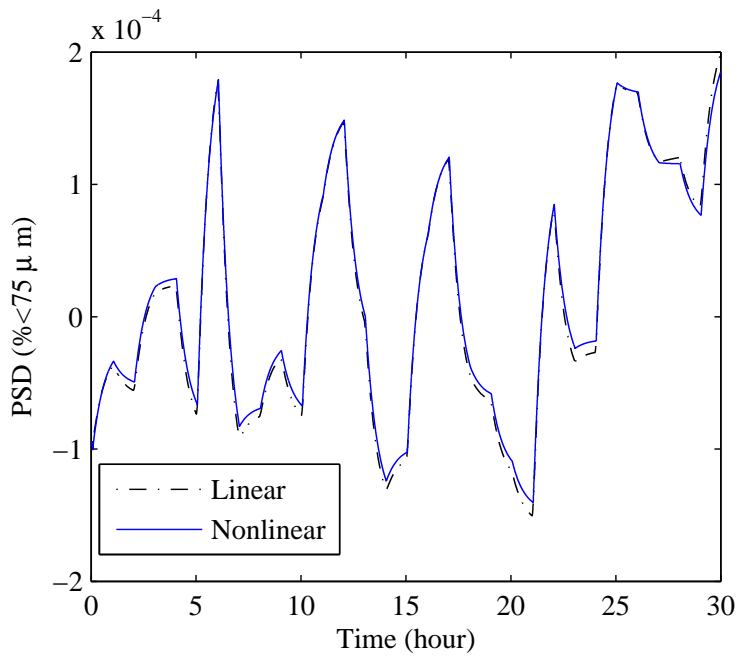

(a) Comparison for the PSD

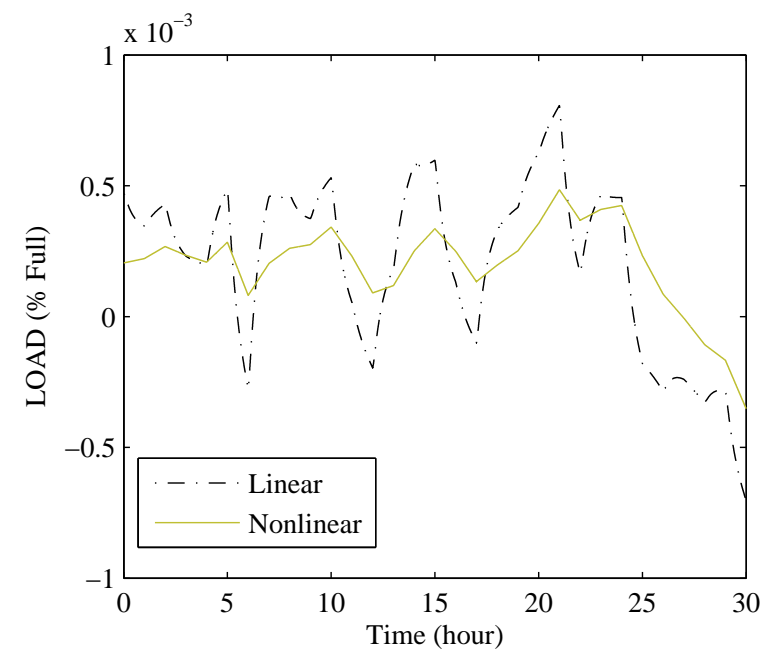

(b) Comparison for the LOAD

Figure 7.4: CVs responses to the multiple step tests of the MFS. No linear relation between the SLEV and MFS is revealed from the system identification based on the relevant step tests and accordingly no comparison is done for them.

to $330 \mathrm{~m}^{3}$ /hour to maintain SLEV. The CFF of $500 \mathrm{~m}^{3} /$ hour might not be able to maintain the PSD at setpoint.

Now assume that the SFW is controlling the PSD and has to change to $400 \mathrm{~m}^{3} /$ hour to maintain the PSD, and then the CFF has to change to $570 \mathrm{~m}^{3} /$ hour to compensate, but it is limited to $500 \mathrm{~m}^{3}$ /hour, therefore, the SLEV will increase until it overflows.

Scenario 2: The hardness of the ore increases that causes the mill to now discharge at 120 $\mathrm{m}^{3}$ /hour. The SFW has to increase to $323 \mathrm{~m}^{3} /$ hour to maintain SLEV at setpoint.

If the SFW was controlling the PSD, it might need to reduce SFW to $220 \mathrm{~m}^{3} /$ hour to achieve 
the correct density to the cyclone. the CFF would then need to change to $340 \mathrm{~m}^{3} /$ hour, but its lower limit is $400 \mathrm{~m}^{3}$ /hour that will cause the sump to run dry, therefore, instability. Even if the SFW is higher at $250 \mathrm{~m}^{3}$ /hour because of a coarser product from the mill requiring a lower density, the CFF will still need to reduce to $370 \mathrm{~m}^{3} /$ hour which it cannot, causing the sump to run dry.

These two scenarios show that the constraints on the SFW will need to be tightened to allow the CFF to maintain SLEV. But as the discharge rate of the mill changes due to changing conditions such as ore hardness and composition, the constraints on the SFW will have to change.

The relative gain array, which is very useful in practice in determining preferable pairings, is used to investigate which one of the above mentioned pairings is better and it turns out that both options of the pairings are not satisfactory from relative gain array viewpoint. The details are given in Appendix A.

\subsubsection{Input-output controllability analysis}

Before various possible control technologies are attempted for milling circuit control, there is one fundamental problem: How well can the circuit be controlled? Input-output controllability provides a measure of the ability to achieve acceptable control performance; that is, to keep the outputs within specified bounds or displacements from their references, in spite of unknown but bounded variations, such as disturbances and plant changes (including uncertainty), using available inputs and available measurements (Skogestad and Postlethwaite, 2005). Input-output controllability is independent of the controller and is a property of a plant. It is intended to find out what control performance can be anticipated. Another term for the input-output controllability is performance targeting. Proper scaling of the input, output and disturbance variables prior to the analysis is essential.

\subsubsection{Scaling of the circuit model}

Scaling is very important in controller design in that it facilitates model analysis and controller design significantly. For this purpose, it is required to make a judgement at the beginning on the expected or allowed magnitudes of inputs, disturbances and reference changes, and allowed deviation for each output (Skogestad and Postlethwaite, 2005). Note that the originally scaled linear model of the process being considered in deviation variables is

$$
\hat{y}=\hat{G} \hat{u}+\hat{G}_{d} \hat{d} ; \quad \hat{e}=\hat{y}-\hat{r}
$$

where the is used to indicate the variables are in originally scaled units. It is useful to scale the variables to be less than 1 in magnitude. It is done by dividing each variable by the relevant maximum expected or allowed change. Regarding the manipulated variables and disturbances, the following variables are used:

$$
d=\hat{d} / \hat{d}_{\max }, \quad u=\hat{u} / \hat{u}_{\max }
$$

Considering that a major objective of control is to minimize control error (setpoints-CVs), the maximum control error is used for the scaling of $r, y, e$ :

$$
y=\hat{y} / \hat{e}_{\text {max }} ; \quad r=\hat{r} / \hat{e}_{\max } ; \quad e=\hat{e} / \hat{e}_{\max }
$$


The following scaling factors are used to formalise the procedure:

$$
D_{e}=\hat{e}_{\max }, \quad D_{u}=\hat{u}_{\max }, \quad D_{d}=\hat{d}_{\max }, \quad D_{r}=\hat{r}_{\max }
$$

Then, there exists

$$
y=G u+G_{d} d ; \quad e=y-r
$$

where

$$
G=D_{e}^{-1} \hat{G} D_{u}, \quad G_{d}=D_{e}^{-1} \hat{G}_{d} D_{d}
$$

The selected scaling factors for the model given in Section 7.2.3.1 are:

$\hat{e}_{\text {max }}=\operatorname{diag}\left(\left[\begin{array}{lll}0.2 & 3 & 0.1\end{array}\right]\right)$;

$\hat{u}_{\text {max }}=\operatorname{diag}\left(\left[\begin{array}{lll}43 & 133 & 100\end{array}\right]\right)$;

$\hat{d}_{\text {max }}=\operatorname{diag}([22.40 .08])$.

\subsubsection{Performance limitations with regard to right-half plane poles and zeros}

Generally, the right-half plane (RHP) poles and zeros will impose fundamental limitations on the achievable control performance (Chen, 2000). The relevant investigation is fairly crucial for the input-output controllability analysis. The poles and zeros of the circuit model are obtained. The poles are $(-1.8519,-4.1667,-1.7241,0,-0.2585,0)$ and zeros are $(-5.3054,-0.0185,-0.6794$, -1.7504). All of them are not in the open right-half of complex plane and accordingly do not imply fundamental control problems (Goodwin et al., 2001).

\subsubsection{Frequency response and relative gain array of the circuit}

The frequency response of the plant model is derived and the relative gain elements are obtained based on the response, as shown in Fig. 7.5. It can be seen that the RGA elements are large in the frequency range essential for the circuit control, implying that the circuit is fundamentally difficult to control owing to strong interactions and sensitivity to uncertainty (Skogestad and Postlethwaite, 2005).

\subsubsection{Singular value decomposition of the circuit model}

Singular values of the circuit model $G(j \omega)$ are derived and shown as function of frequency in Fig. 7.6. The minimum singular value, $\underline{\sigma}(G(j \omega))$, is a useful controllability measure. It preferably is as large as possible over the frequency range crucial for control. However, this is not true in this case, the $\underline{\sigma}(G(j \omega))$ being 0.007 over the range $[0.01,100] \mathrm{rad} / \mathrm{sec}$.

\subsubsection{Functional controllability of the circuit}

For a plant $\mathrm{G}(s)$ with $l$ outputs, $r$ can be used to indicate the normal rank of $\mathrm{G}(s)$. The normal rank of $\mathrm{G}(s)$ is the rank of $\mathrm{G}(s)$ at all values of $s$ except at limited number of singularities. In 


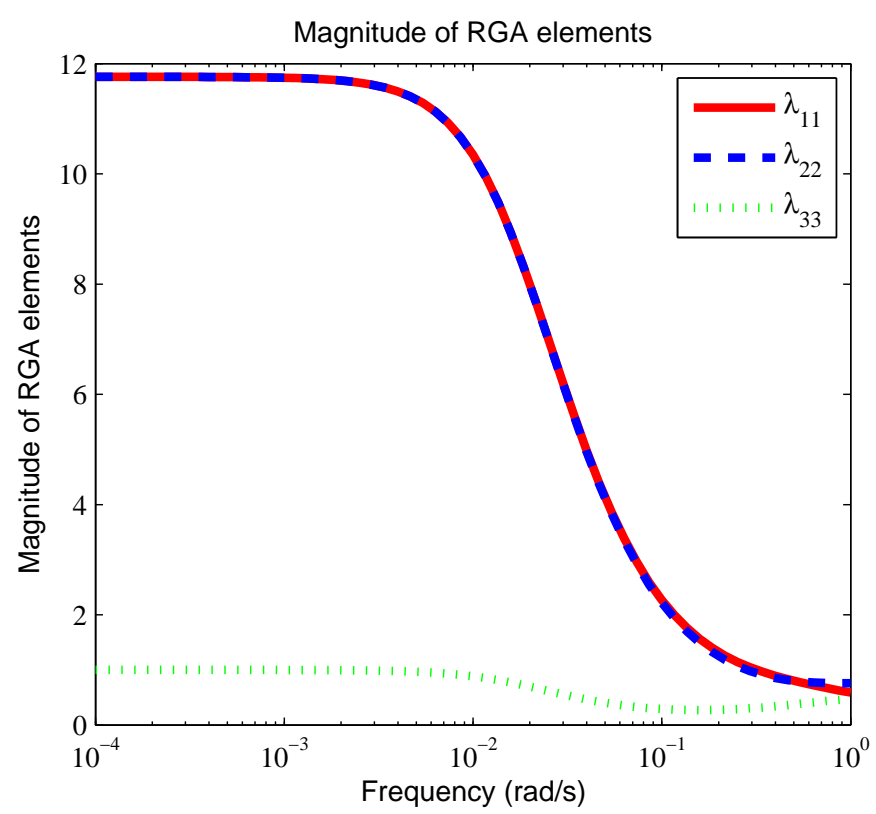

Figure 7.5: Relative gain array elements of the circuit model

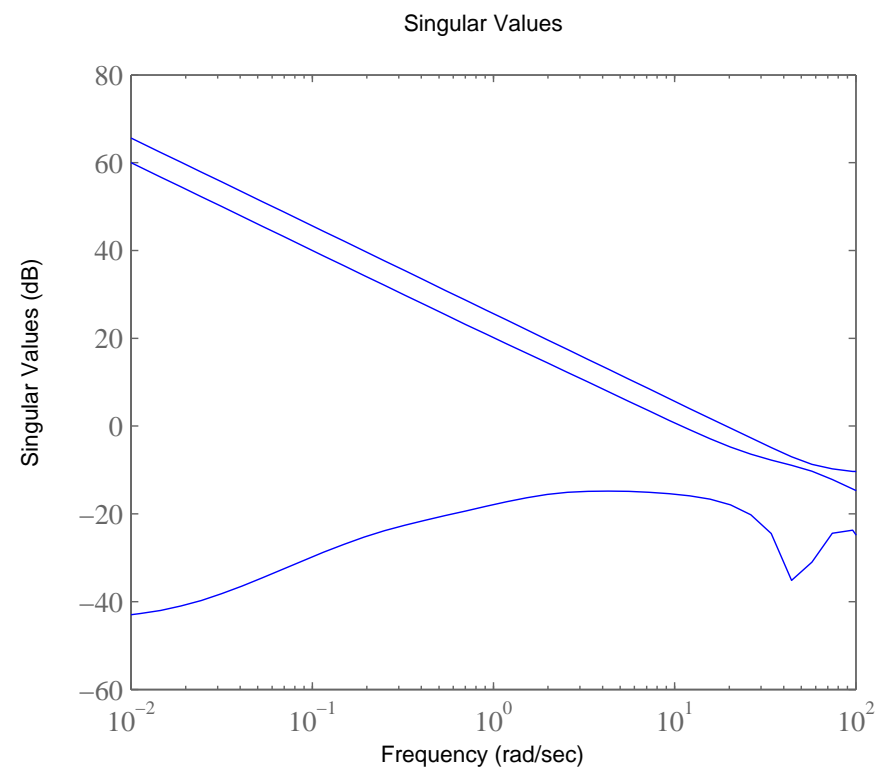

Figure 7.6: Singular values as function of frequency for the circuit model $G$ 
order to control all outputs independently, $r$ is required to equal $l$ (meaning the plant has full rank) and then the plant can be named 'functionally controllable'. For a given value of $s$, if the minimum singular value is nonzero, then $\mathrm{G}(s)$ has full rank. Considering the singular values given above, the minimum singular values are all nonzero. Therefore, the $\mathrm{G}(s)$ has full rank at all frequencies and the circuit is functional controllable.

\subsubsection{Condition number of the circuit model}

The condition number of $\mathrm{G}(s)$ is defined as the maximum singular value divided by the minimum singular value:

$$
\gamma(G) \triangleq \bar{\sigma}(G) / \underline{\sigma}(G)
$$

The condition number is often used as an input-output controllability measure. A small condition number usually implies that the multivariable impact of uncertainty is not likely to be serious. A large condition number may mean strong sensitivity to unstructured input uncertainty (Skogestad and Postlethwaite, 2005). In this case, the maximum singular value is 1908 and the minimum singular value 0.0071 ; accordingly the condition no is $2.69 \mathrm{e} 5$, implying poor controllability.

\subsubsection{Performance requirements imposed by disturbances}

Generally large and fast disturbances entail aggressive control and a large bandwidth (Goodwin et al., 2001). When considering the performance limitations associated with disturbances in MIMO control issues, directions play a key role. For one single disturbance with the vector $g_{d}$ representing its impact on outputs $\left(y=g_{d} d\right)$, the relevant disturbance direction is defined by

$$
y_{d}=\frac{g_{d}}{\left\|g_{d}\right\|_{2}}
$$

Note that $g_{d}$ is a vector in $G_{d}$. Since disturbances and outputs have both been scaled, the worst disturbance may be chosen as $|d(w)|=1$ and the disturbance rejection objective is that at each frequency the 2-norm of the error be less than 1, namely, $\|e(w)\|_{2}<1$. For feedback control $e=S g_{d} d$, the objective is satisfied when

$$
\left\|S g_{d}\right\|_{2}=\bar{\sigma}\left(S g_{d}\right)<1 \quad \forall w
$$

This formula can be used to derive bounds on the sensitivity function $S$. Incorporating Eq. (7.49) and (7.50), there exists

$$
\left\|S y_{d}\right\|_{2}<1 /\left\|g_{d}\right\|_{2} \quad \forall w
$$

Then bounds regarding to $S$ can be given as

$$
\underline{\sigma}(S)\left\|g_{d}\right\|_{2} \leq\left\|S g_{d}\right\|_{2} \leq \bar{\sigma}(S)\left\|g_{d}\right\|_{2}
$$

Since $\underline{\sigma}(S)=1 / \bar{\sigma}(I+L)$, it can be derived that for acceptable performance it is necessary to ensure $\bar{\sigma}(I+L)$ is larger than $\left\|g_{d}\right\|_{2}$ in the controller design (Skogestad and Postlethwaite, 
2005). For the milling circuit being considered, the scaled disturbance transfer function matrix is

$$
G_{d}=\left[\begin{array}{cc}
\frac{-26.88}{(1+0.54 s)} e^{(-0.014 s)} & \frac{(0.024-2.4 e-5 s)}{(1+4.24 s)} e^{(-0.014 s)} \\
\frac{0.014}{(1+14.9 s)} e^{(-0.438 s)} & \frac{4.67-5}{\left(1+2(4.5)(14) s+(14 s)^{2}\right)} e^{(-0.014 s)} \\
\frac{129.9}{(1+1.41 s)} e^{(-0.089 s)} & \frac{-0.0048}{\left(1+2(2.90)(6.25) s+(6.25 s)^{2}\right)(1+33.4 s)} e^{(-0.014 s)}
\end{array}\right]
$$

$g_{d}$ refers to each of the two columns of $G_{d}$.

\subsubsection{Control performance specifications based on economic objec- tives}

The economic objectives of milling circuits are to produce a product, at a reasonable throughput, from which good recovery can be achieved in the downstream processes, and to reduce operating cost (Lo et al., 1996; Hulbert, 1989, 2002). The objectives are often broken down into grind fineness increase, throughput increase, better recovery, etc (Sosa-Blanco et al., 2000). In this chapter the strategy is to maximise throughput while maintaining the PSD at a certain point. The benefits of better control will then result from decreasing the variations of the controlled variables and then moving the operating point closer to the setpoint (Marlin et al., 1991; Muske and Finegan, 2002).

In what follows, $80 \%$ passing $75 \mu \mathrm{m}$ is used as the setpoint for the PSD for several reasons. The PSD setpoint is usually selected based on a trade-off that too fine a grind maximises the mineral liberation at the expense of low throughput and high energy cost, whereas too coarse a grind decreases concentrate grade because of the presence of middlings (Wills and NapierMunn, 2006; Hodouin et al., 2001). Maximising the net revenue of the recovered mineral tends to decrease metallurgical performance and increase the loss of valuable minerals (low recovery) to the plant tailings, which has ethical implications for resource conservation and environmental concerns (Sosa-Blanco et al., 2000). Theoretically, the PSD setpoint should be selected based on the relation between recovery and grinding circuit product PSD. However, practically, the narrowest PSD in the size range of maximum recovery is obtained at the design or tuning stage of the grinding circuit, and the operation is controlled at a single target (Hodouin et al., 2001). The setpoints of the LOAD and SLEV are $45 \%$ and $50 \%$ full respectively.

In the control problem in this chapter, a distinction is made between the functional and economic performance. The functional performance refers to setpoint tracking and disturbance rejection; it will be evaluated in terms of whether the CVs follow the setpoints under various disturbances and how much the variances are. The economic performance refers to the mineral recovery obtained in the downstream flotation circuit. Although they are different from a conceptual viewpoint, there is a natural transition and consistency between them, i.e., the functional specifications are determined based on the economic objectives. In the following sections, the PID controllers and NMPC controller will be designed to fulfil the control strategy.

\subsection{Framework step 1: Base case identification}

The first step when considering a control upgrade project is to analyse the performance of the current system, often referred to as the base case. This step needs in-depth understanding of 
the process being considered, such as the process dynamics, economics, operating conditions, operating constraints, etc. It also needs careful selection of the operating data, which should reflect the typical operation of the process considered.

The base case in this work is the scenario where the milling circuit is controlled by three singleloop PID controllers. PID control is used here, as most milling circuits in industry are controlled in this way (Wei and Craig, 2009c). The controller design, relevant functional performance and economic performance are then discussed. A decentralised controller designed after decoupling the system model is also investigated and its performance is compared to that for the singleloop PID controllers. It is then illustrated that an advanced controller needs to be considered to specifically improve disturbance rejection.

\subsubsection{Proportional-integral-derivative controllers design}

Single-loop PID controllers used in Coetzee et al. (2008) are used in the base case to realize the economic objective elaborated on in Section 7.2.5. Linear models described in Section 7.2.3 are used for the PID controller design, whereas the interaction between loops is ignored as they cannot be handled in the PID controllers design used in this subsection. The nonlinear model described in Section 7.2.1 is used as the plant. The three PID controllers control the PSD-CFF, LOAD-MFS and SLEV-SFW loops. The PID controllers were tuned using the Skogestad Internal Model Control (SIMC) design procedure described in Skogestad (2003). This procedure has two steps, firstly obtaining a first- or second-order plus delay model using a proposed 'half-rule' and then deriving PID controller settings using proposed analytical rules. The PID controllers designed using this procedure generally yield better performance in terms of disturbance rejection and setpoint tracking compared to the traditional PID-tuning methods (Skogestad, 2003). Specifically, disturbance rejection is improved significantly for integrating processes, which is applicable to this chapter, as the LOAD-MFS and SLEV-SFW transfer functions are integrators for the milling circuit examined.

\subsubsection{Functional performance of the proportional-integral-derivative controllers}

The milling circuit controlled by the PID controllers is simulated using two disturbance scenarios. The simulation period of 24 hours is selected in order to give the system sufficient time to respond to disturbances. In the simulation (and also the simulations that will be conducted in the sequel of this chapter), the nonlinear model is used as the process in order to introduce the disturbances in a unifying way and perform as 'fair' as possible comparison of performance related to different controllers designed for the circuit. The parameters shown in Table 7.4 are used, modelled as uniform distributions within the constraints. The most significant uncertainties for the ROM milling circuit are the rock hardness and the rock fraction in the mill feed ore. Both of them are employed in order to make the simulation more realistic.

Firstly, only stationary centred disturbances with the maximum range of uncertainties are used for simulating the nonlinear model. The (white) disturbances follow uniform distributions. The time trends of CVs and MVs are given in Fig. 7.7. It can be seen that the SIMC PID controllers can control the CVs well. Both the SLEV and LOAD are controlled well. Furthermore, the 
MVs do not saturate. The summary of the simulation results is given in Table 7.5.
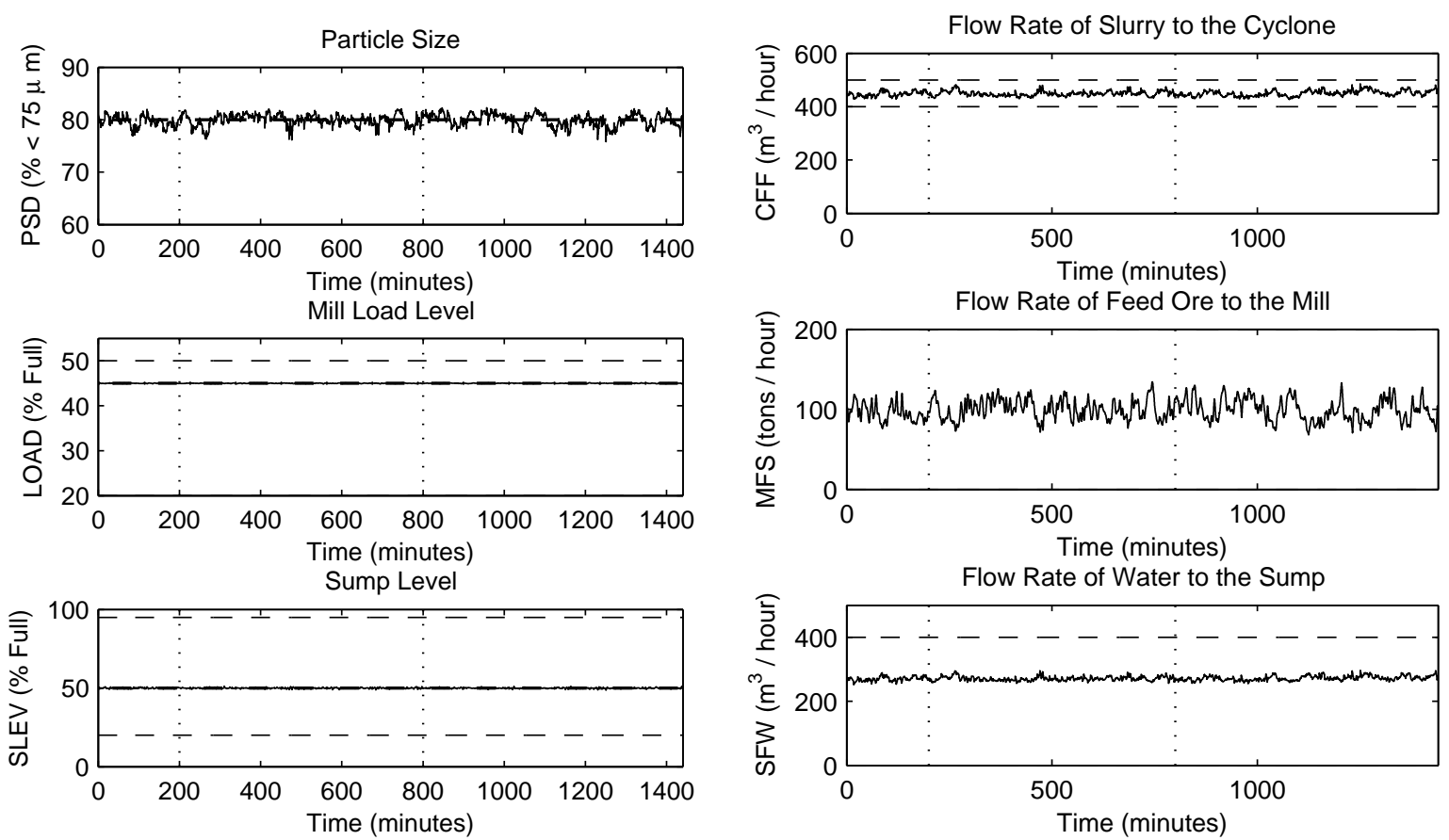

(a) CVs

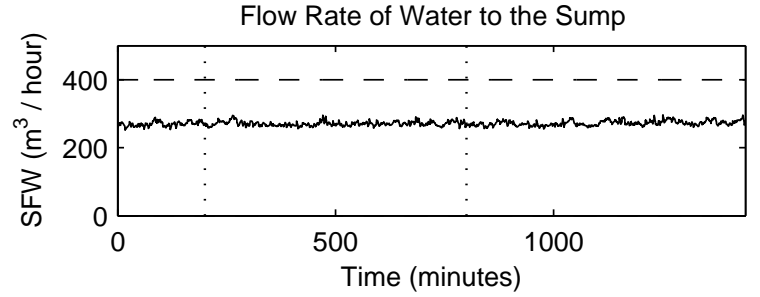

(b) MVs

Figure 7.7: CVs and MVs with SIMC PID controllers with stationary centred disturbance

Table 7.5: Summary of simulation results with stationary centred disturbances under SIMC PID controllers

\begin{tabular}{ccc}
\hline Controlled variable & $\mu_{y_{i}}$ & $\sigma_{y_{i}}$ \\
\hline PSD $(\%<75 \mu \mathrm{m})$ & 79.83 & 0.82 \\
LOAD $(\%$ full $)$ & 45.00 & 0.04 \\
SLEV $(\%$ full $)$ & 49.99 & 0.27 \\
\hline
\end{tabular}

Then in another simulation both persistent and stationary disturbances are applied. The ore hardness is increased by $80 \%$ at time 200 minutes until the end of simulation and the rock fraction is increased by $90 \%$ at time 800 minutes until the end of simulation. The disturbance sizes are selected based on the investigation of the disturbance scenarios used in for example Hulbert et al. (1990), Yahmedi et al. (1998) and Coetzee et al. (2009). Note that both the hardness and rock fraction disturbances have two components here. One is the base level, or alternatively speaking the average level, and the other is a random variation around the base level. The disturbances are large but not uncommon in practice.

The simulation results are shown in Fig. 7.8. It can be seen that the LOAD and SLEV are controlled well, as the dynamics associated with them are relatively simple. From time 200 minutes the PSD drops owing to the increase in ore hardness. After time 800 minutes the PSD slowly drifts back to setpoint as the increase in ore hardness is now countered by the increase in rock fraction. The PSD-CFF controller is not able to maintain the PSD at setpoint as CFF is saturated at its upper limit of $500 \mathrm{~m}^{3} / \mathrm{h}$ from just after time 200 minutes.

The time series of the CVs described in this chapter are assumed to follow Gaussian distributions. Skewness and kurtosis tests confirmed that the time series of the controlled variables reported on in this chapter follow Gaussian distributions (Tabachnick and Fidell, 2001). When 


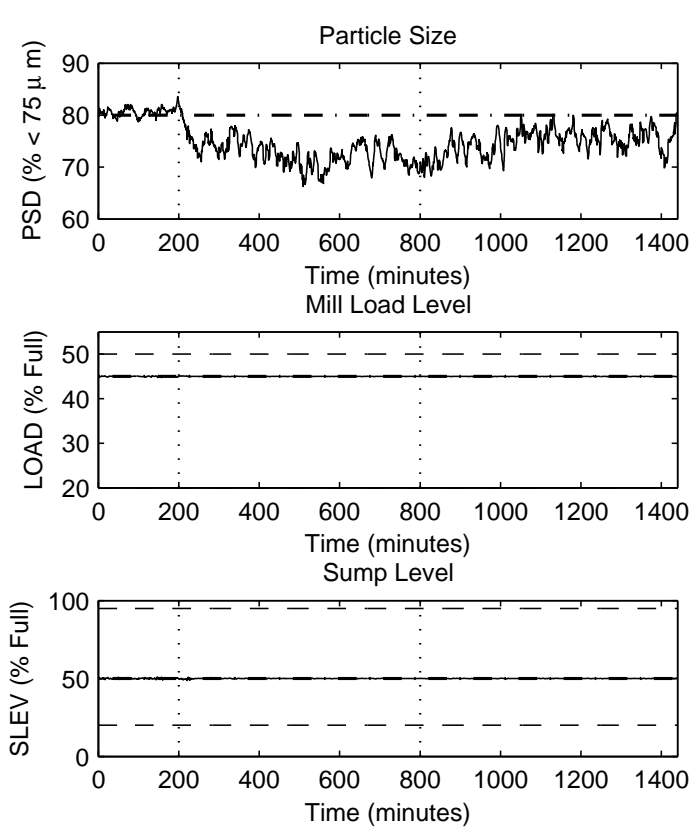

(a) CVs
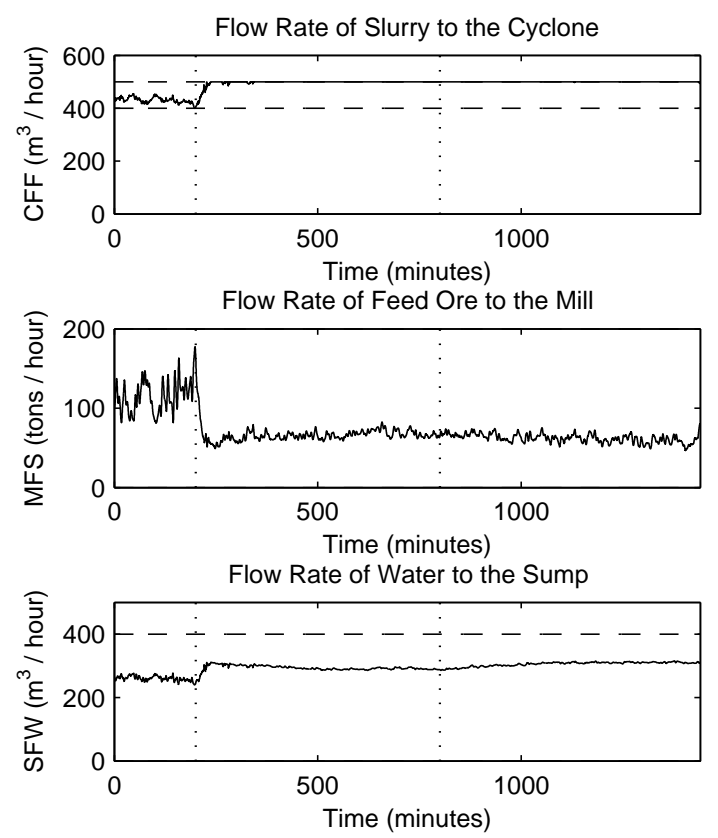

(b) MVs

Figure 7.8: CVs and MVs of one-day operation for the SIMC PID controllers under persistent and random disturbances

the $\mu_{y_{i}}$ and $\sigma_{y_{i}}$ are used to represent the mean and standard deviation of the time series of $y_{i}$, the PDF can be depicted as follows:

$$
f\left(y_{i}\right)=\frac{1}{\sqrt{2 \pi} \sigma_{y_{i}}} \exp \left\{-\frac{\left(y_{i}-\mu_{y_{i}}\right)^{2}}{2 \sigma_{y_{i}}^{2}}\right\}
$$

The PDFs of the CVs are obtained in terms of average values and standard deviations, as shown in Table 7.6.

Table 7.6: Summary of simulation results with stationary centred and persistent disturbances under SIMC PID controllers

\begin{tabular}{ccc}
\hline Controlled variable & $\mu_{y_{i}}$ & $\sigma_{y_{i}}$ \\
\hline PSD $(\%<75 \mu \mathrm{m})$ & 75.05 & 3.94 \\
LOAD $(\%$ full $)$ & 45.00 & 0.04 \\
SLEV $(\%$ full $)$ & 49.99 & 0.17 \\
\hline
\end{tabular}

\section{Attempt to improve the PSD performance by relaxing control of SLEV}

It can be seen from Fig. 7.8 that the PID tunings for the SLEV and LOAD are tight, which does not give room to system to match the PSD setpoint. However, the LOAD needs to be controlled tightly, especially when some optimisation scheme is used (Craig et al., 1992b). For the SLEV, it is considered that relaxed control may give room for SFW to absorb part of the impact of disturbances to the system and help to improve the performance of the PSD. Error squared control is used for this purpose, in which a special nonlinear operator is applied to the input of the SLEV controller (Åström and Hägglund, 1995). The input error is multiplied by its absolute value, leading to the gain dependent on the magnitude of the deviation of the SLEV from its setpoint, as done in Hulbert et al. (1990). The error squared controller is effective in that the buffering potential of the sump is used sufficiently and control of the SLEV is accomplished with small and relatively smooth changes in the control action. 
The best tuned error squared controller results in the simulation results shown in Fig. 7.9 and the statistics of the results are given in Table 7.7. It can be seen that, compared to the results shown in Fig. 7.8 and Table 7.6, the control of SLEV is relaxed. However, the sacrifice of the SLEV control does not help improve the PSD performance as the disturbance is persistent. The error squared control just smooths out SFW, but it has no effect on the PSD due to a disturbance of hardness or rock fraction. The only way to counter the disturbance is to change the CFF, which then runs into a constraint and loses control of the PSD, or change SFW to change density, but then the CFF has to be changed as well to maintain the SLEV. The point is that the proper PSD control is a multivariable problem when constraints are involved, and good control cannot be achieved with the single-loop PID controllers. This multivariable control problem will be considered as a major function of the advanced controller that will be investigated in the following sections.

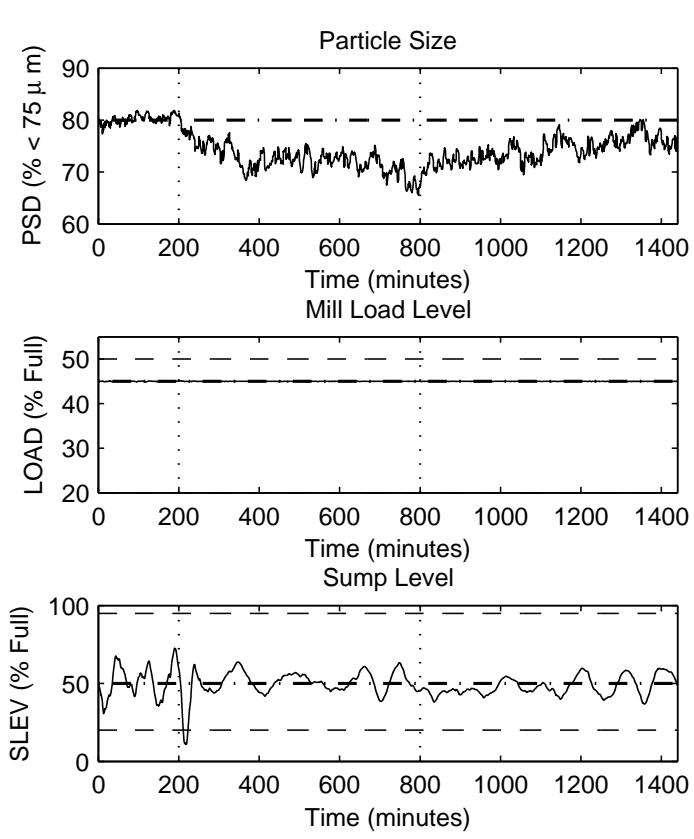

(a) CVs

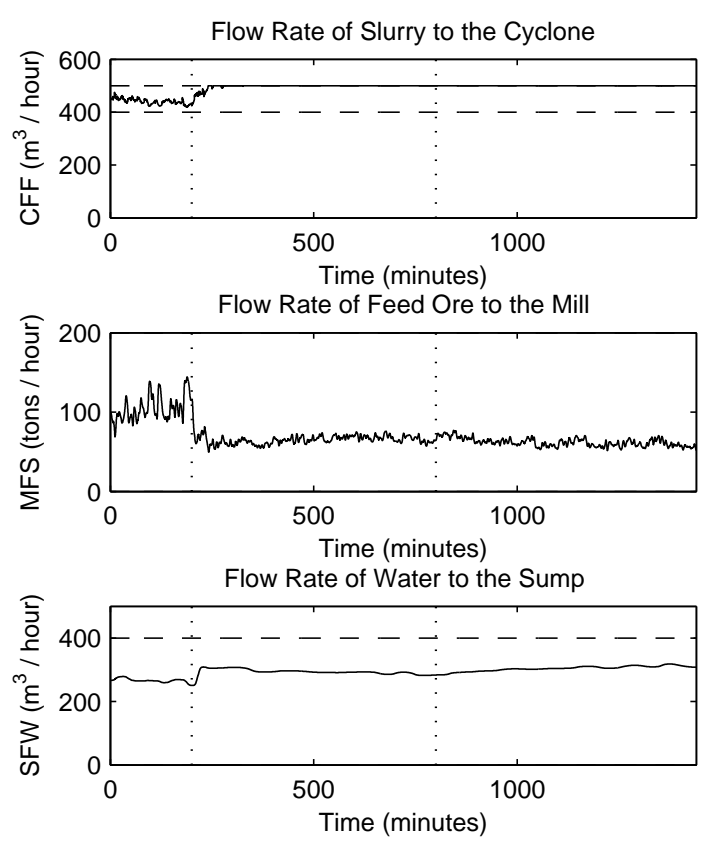

(b) MVs

Figure 7.9: CVs and MVs of one-day operation for the revised SIMC PID controllers with error squared control for the SLEV under persistent and random disturbances

Table 7.7: Summary of simulation results with stationary centred and persistent disturbances under SIMC PID (and error squared) controllers

\begin{tabular}{ccc}
\hline Controlled variable & $\mu_{y_{i}}$ & $\sigma_{y_{i}}$ \\
\hline PSD $(\%<75 \mu \mathrm{m})$ & 74.96 & 3.55 \\
LOAD $(\%$ full $)$ & 45.00 & 0.03 \\
SLEV $(\%$ full) & 49.65 & 5.99 \\
\hline
\end{tabular}

\subsubsection{Economic performance of the base case}

The preferred way to indicate economic performance is by integrating the product of a PF and PDF of a time series of a process variable over a period of interest (Zhou, 2002; Wei et al., 2007; Oosthuizen et al., 2004), as described in Section 6.5. 
Both the LOAD and SLEV are controlled well and do not deviate from the constraints and thus the relevant economic impact is null. The fluctuations of the PSD around the setpoint cause fluctuations in flotation circuit recoveries, which can be economically significant. The overall EPI (as described in Section 6.5) is therefore equal to the EPI associated with the PSD, which is

$$
\begin{aligned}
\Psi_{y_{1}} & =\int_{-\infty}^{\infty} v\left(y_{1}\right) f\left(y_{1}\right) d y_{1} \\
& =\int_{-\infty}^{\infty}\left(\frac{1}{\sqrt{2 \pi} \sigma_{y_{1}}} \exp \left\{-\frac{\left(y_{1}-\mu_{y_{1}}\right)^{2}}{2 \sigma_{y_{1}}^{2}}\right\}\right)\left(a y_{1}^{2}+b y_{1}+c\right) d y_{1}
\end{aligned}
$$

where $a=-0.009776, b=1.705$ and $c=-2.955$. Generally it takes 20-40 minutes for a change in the PSD of the feed to the flotation circuit impacts on the recovery (This info comes from an industrial connection). Note that, when the convolution is used, it is based on the steadystate relation between the PSD and the recovery that occurs after the time delay. The essence of convolution is the inherent relationship, and it does not necessarily requires the involved functions work simultaneously. The PDF-PF convolution method will be used each time the recovery is obtained. The performance is assessed based on both mean values and variations of controlled variables.

It can be seen from the PF of the PSD shown in Fig. 6.4 that the optimal operating point for the PSD is $87.2 \%$ and the relevant optimal recovery is $71.4 \%$. The economic performance of the base case is derived by substituting the values given in Table 7.6 into Eq. (7.55) over the simulation period; the average base case recovery of the downstream flotation circuit is $69.61 \%$. For the purpose of this study, if the product of the throughput rate, time period, head grade and price of the recovered mineral is noted as $k$, then the recovered mineral value is

$$
\begin{aligned}
\text { Value } & =(\text { throughput rate }) \times(\text { time period }) \times(\text { head grade }) \times(\text { recovery }) \times(\text { mineral price }) \\
& =\text { recovery } \times k
\end{aligned}
$$

according to Eq. (6.1). The value of recovered mineral of the base case is therefore $(69.61 \mathrm{k}) \%$. For any given circuit, the actual mineral value can be obtained by substituting the values of throughput rate, time period, mineral price and head grade into Eq. (7.56).

\subsubsection{Performance bound}

The reduction of the variation of CVs can often produce economic improvement as long as the performance functions are not linear and unconstrained. However, there is a theoretical limit to the reduction in the variance for any given process, known as minimum variance. The minimum variance based performance index is defined as:

$$
\eta=\sigma_{m v}^{2} / \sigma_{y}^{2}
$$

where $\sigma_{m v}^{2}$ is the minimum variance of a process variable and $\sigma_{y}^{2}$ is the variance of the process variable based on the data collected over a period of interest. The range of $\eta$ is between 0 and 1 . When $\eta$ is close to 1 , the variance of the CV considered is already near the theoretical minimum and there is not much room to improve the $\mathrm{CV}$ performance from the viewpoint of variance reduction. When $\eta$ is close to 0 , the $\mathrm{CV}$ variance can be reduced significantly. 
The minimum variance can be obtained from process data and a process model. The data generated in Section 7.3.2 and the linearised transfer function model of the circuit are used for the calculation of the minimum variance. Since the focus of economic performance is mainly on the PSD here, $\eta$ of only the PSD is derived. Here, the minimum variance is calculated using the filtering and correlation analysis algorithm given in Huang and Shah (1999); refer to Appendix B. The $\eta$ obtained is 0.01 . Considering the standard deviation of the PSD of the base case is 3.94, the minimum variance for the PSD is obtained as $0.15\left(0.39^{2}\right)$, using Eq. (7.57). Minimum variance control can therefore theoretically reduce the standard deviation by about 90\%. Furthermore, with this minimum variance and the assumption that the controller works perfectly and the PSD operating point is $80 \%$, the best achievable average recovery (EPI) can be derived as $70.69 \%$ using Eq. (7.55), an improvement of $1.08 \%$ over the base case, and accordingly the value of the mineral recovered is $(70.69 \mathrm{k}) \%$.

\subsubsection{Decentralised controller based on decoupling}

A conceptually simple method to perform multivariable control is a two-step procedure in which a 'compensator' $(W(s))$, that deals with the interactions in process model $(G(s))$ is designed first and then a diagonal controller $(K(s))$ is designed using methods for single-input singleoutput systems (Vázquez and Morilla, 2002; Skogestad and Postlethwaite, 2005). Then the shaped plant is $G_{s}(s)=G(s) W(s)$ and the overall controller is $W(s) K(s)$. In this subsection, a decoupling controller and another set of PID controllers are designed based on decoupling the milling circuit's model to match the economic objective described in Section 7.2.5. There are three options to perform decoupling: dynamic decoupling for which $G_{s}(s)$ is diagonal for all frequencies, steady-state decoupling for which $G_{s}(0)$ is diagonal and approximate decoupling at frequency $\omega_{0}$ for which $G_{s}\left(j \omega_{0}\right)$ is as diagonal as possible. Dynamic decoupling is used in this case.

\subsubsection{Theory used for the decoupling}

Among various decoupling methods, lineal decoupling described in Deshpande (1989) is used most (Vázquez and Morilla, 2002) and also used here. The decoupler is designed for a $2 \times 2$ system

$$
G(s)=\left[\begin{array}{ll}
G_{11}(s) & G_{12}(s) \\
G_{21}(s) & G_{22}(s)
\end{array}\right]
$$

The structure of the control system is given in Fig. 7.10.

Four controllers are used, namely two conventional feedback controllers $G_{c 1}$ and $G_{c 2}$ and two decouplers $T_{12}$ and $T_{21}$. The output signal of each feedback controller is the input signal of a decoupler. The decouplers are designed to counteract process interactions. Specifically in this case, decoupler $T_{12}$ is designed to cancel $Y_{12}$ and $T_{21}$ designed to cancel $Y_{21}$. For this purpose, the following equation needs to hold

$$
\left(G_{p 21}(s)+G_{p 22}(s) T_{21}(s)\right) U_{11}(s)=0
$$




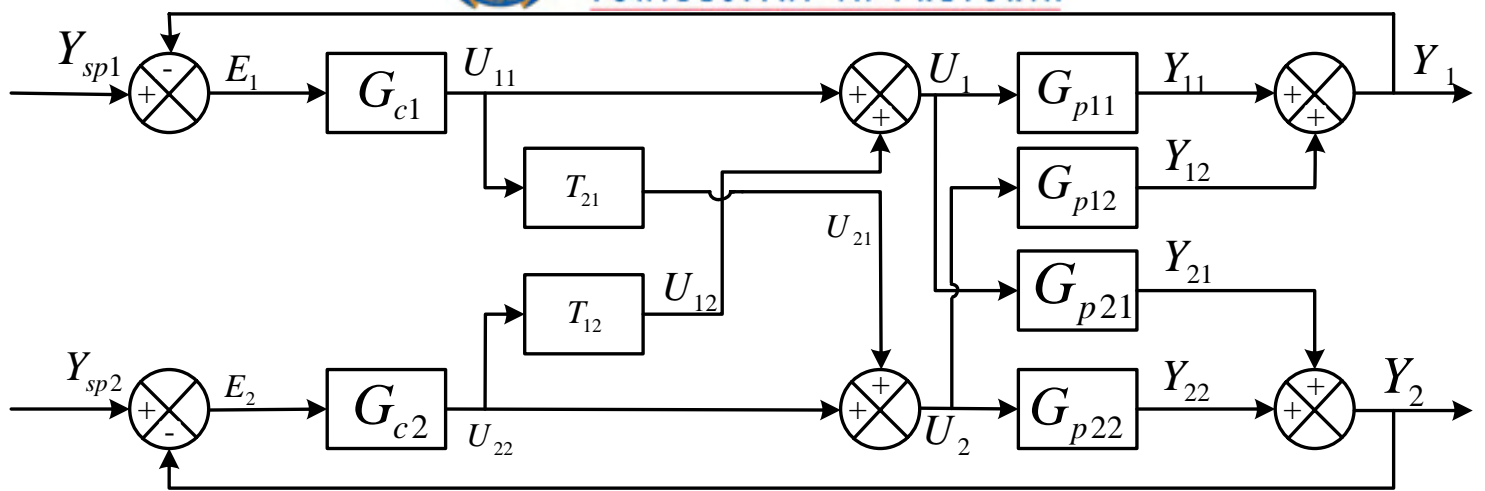

Figure 7.10: The scheme of the decoupling system (Seborg et al., 2004)

and accordingly

$$
T_{21}(s)=-\frac{G_{p 21}(s)}{G_{p 22}(s)}
$$

Similarly, the decoupler $T_{12}$ needs to satisfy

$$
T_{12}(s)=-\frac{G_{p 12}(s)}{G_{p 11}(s)}
$$

Subsequently, the dynamics between $Y$ and $U$ is given by

$$
\begin{aligned}
{\left[\begin{array}{l}
Y_{1}(s) \\
Y_{2}(s)
\end{array}\right] } & =\left[\begin{array}{ll}
G_{11}(s) & G_{12}(s) \\
G_{21}(s) & G_{22}(s)
\end{array}\right]\left[\begin{array}{c}
U_{1}(s) \\
U_{2}(s)
\end{array}\right] \\
& =\left[\begin{array}{ll}
G_{11}(s) & G_{12}(s) \\
G_{21}(s) & G_{22}(s)
\end{array}\right]\left[\begin{array}{cc}
1 & T_{12}(s) \\
T_{21}(s) & 1
\end{array}\right]\left[\begin{array}{c}
U_{11}(s) \\
U_{22}(s)
\end{array}\right] \\
& =\left[\begin{array}{cc}
G_{11}(s)+G_{12}(s) T_{21}(s) & 0 \\
0 & G_{21}(s) T_{12}(s)+G_{22}(s)
\end{array}\right]\left[\begin{array}{c}
U_{11}(s) \\
U_{22}(s)
\end{array}\right]
\end{aligned}
$$

It can be seen that with the decouplers the system is diagonal. Given that, it will be natural to use traditional PID controllers for the decoupled system.

\subsubsection{Decoupling of the milling circuit}

For this milling circuit, the interactions mainly concern the cyclone and sump models. Besides the diagonal elements in the circuit's overall transfer function matrix model that are used for the SIMC PID controllers given in Section 7.3.1, the interactions of the cyclone and sump models are taken into account and a decoupler is designed. The resulting model for the cyclone and sump is

$$
\left[\begin{array}{c}
\operatorname{PSD}(s) \\
\operatorname{SLEV}(s)
\end{array}\right]=\left[\begin{array}{cc}
-0.00035 \frac{(1-0.63 s)}{(1+0.54 s)} e^{(-0.011 s)} & \frac{0.00055}{(1+0.24 s)} e^{(-0.011 s)} \\
\frac{-0.29}{s} & \frac{0.42}{s}
\end{array}\right]\left[\begin{array}{c}
\operatorname{CFF}(s) \\
\operatorname{SFW}(s)
\end{array}\right]
$$

According to Eq. (7.61), $T_{12}(s)$ is

$$
T_{12}(s)=\frac{1.57(1+0.54 s)}{(1+0.24 s)(1-0.63 s)}
$$


However, non-minimum phase zeros (here relating to $(1-0.63 s)$ ) cannot be inverted (Goodwin et al., 2001; Skogestad and Postlethwaite, 2005) and an acceptable compromise is to use its mirror image $(1+0.63 s)$ (Fallside, 1977). Therefore

$$
T_{12}(s)=\frac{1.57(1+0.54 s)}{(1+0.24 s)(1+0.63 s)}
$$

According to Eq. (7.60), $T_{21}(s)$ is

$$
T_{21}(s)=0.69
$$

\subsubsection{Design of the decentralised controller using singular frequency-based tech- nique}

Based on the decoupled system, single-loop PID controllers are designed using frequency-based tuning techniques. The Simulink scheme for this decentralised controller is given in Fig. 7.11. The 'Sisotool' in the Control System Design Toolbox of Matlab is used. Performance metrics, such as integral absolute error, integral square error, integral time absolute error and integral time square error are all used to derive the PID controllers parameters and then to investigate how the decentralised controller works under different disturbance scenarios. Several controller structures can be selected for each performance metric with this tool. After a number of trials it turns out that, for both $G_{c 1}$ and $G_{c 2}$, the best tuning among all possibilities is relevant to the integral absolute error performance metric. Then these two PID controllers are tuned further. 


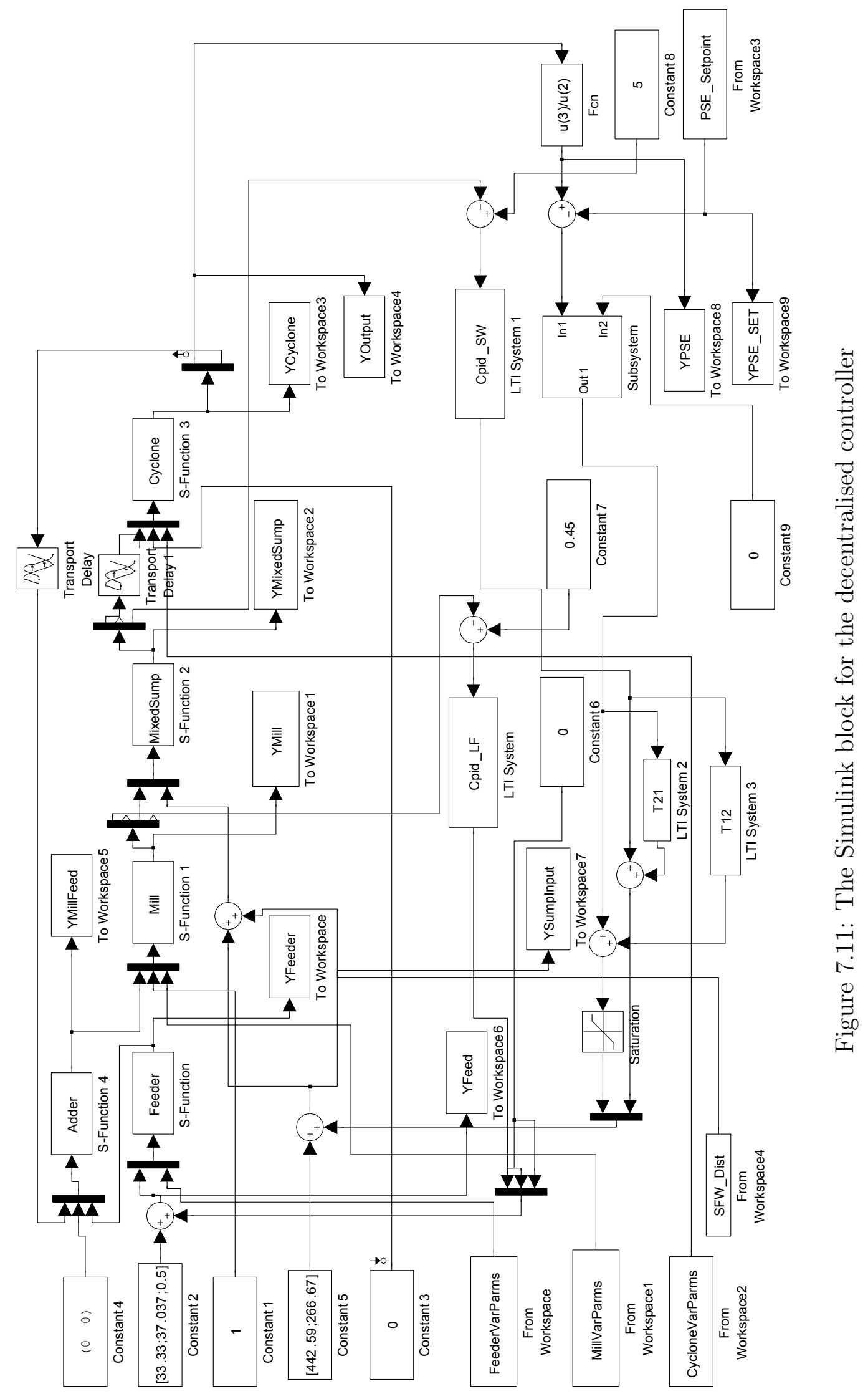




\subsubsection{Performance of the decentralised controller}

In this subsection, the same disturbance scenarios as used in Section 7.3.2 are adopted for the decentralised controller. Firstly, only stationary centred disturbances are applied. The time trends of CVs and MVs for the decentralised controller are given in Fig. 7.12. It can be seen that with the stationary centred disturbances the decentralised controller achieves smaller variation in the PSD, compared to the SIMC PID controllers. The SLEV and LOAD are both controlled well. In addition, the system is not driven to the constraints. The summary of the simulation results is given in Table 7.8 . The recovered mineral values are $(70.65 \mathrm{k}) \%$. There is no big advantage for the decentralised controller over the SIMC PID controllers under this disturbance scenario.
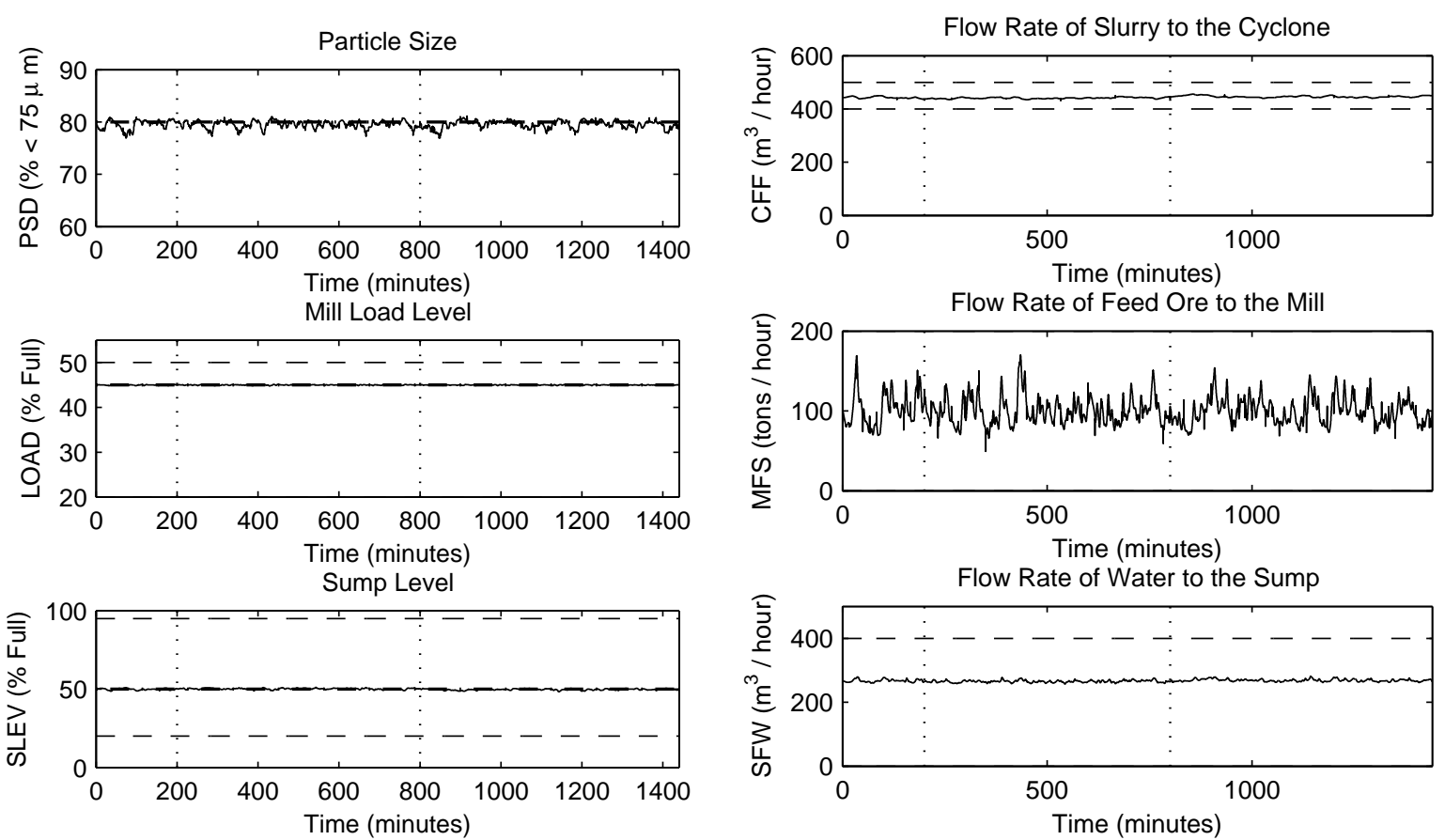

Flow Rate of Water to the Sump

(a) $\mathrm{CVs}$

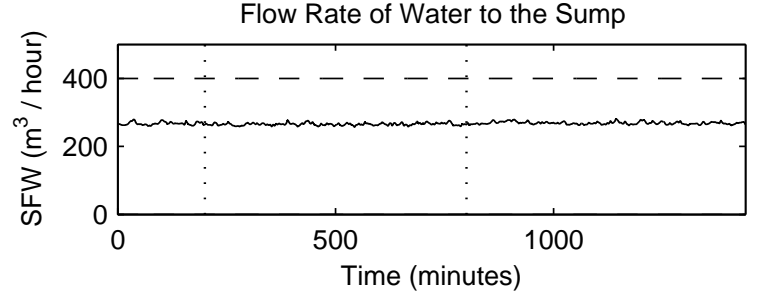

(b) MVs

Figure 7.12: CVs and MVs with stationary centred disturbance for the decentralised controller

Table 7.8: Summary of simulation results with stationary centred disturbances under the decentralised controller

\begin{tabular}{ccc}
\hline Controlled variable & $\mu_{y_{i}}$ & $\sigma_{y_{i}}$ \\
\hline PSD $(\%<75 \mu \mathrm{m})$ & 79.78 & 0.61 \\
LOAD $(\%$ full $)$ & 45.00 & 0.06 \\
SLEV (\% full) & 51.04 & 0.37 \\
\hline
\end{tabular}

Then both the persistent and stationary disturbances are applied for the decentralised controller. The time trends of CVs and MVs are given in Fig. 7.13 and a summary is given in Table 7.9. It can be seen that the decentralised controller provides better disturbance rejection performance than the SIMC PID controllers, regarding the results given in Section 7.3.2. The CFF saturates later for the decentralised controller. The recovered mineral value is $(70.36 \mathrm{k}) \%$, which is better than $(69.61 \mathrm{k}) \%$ achieved by the SIMC PID controllers. 

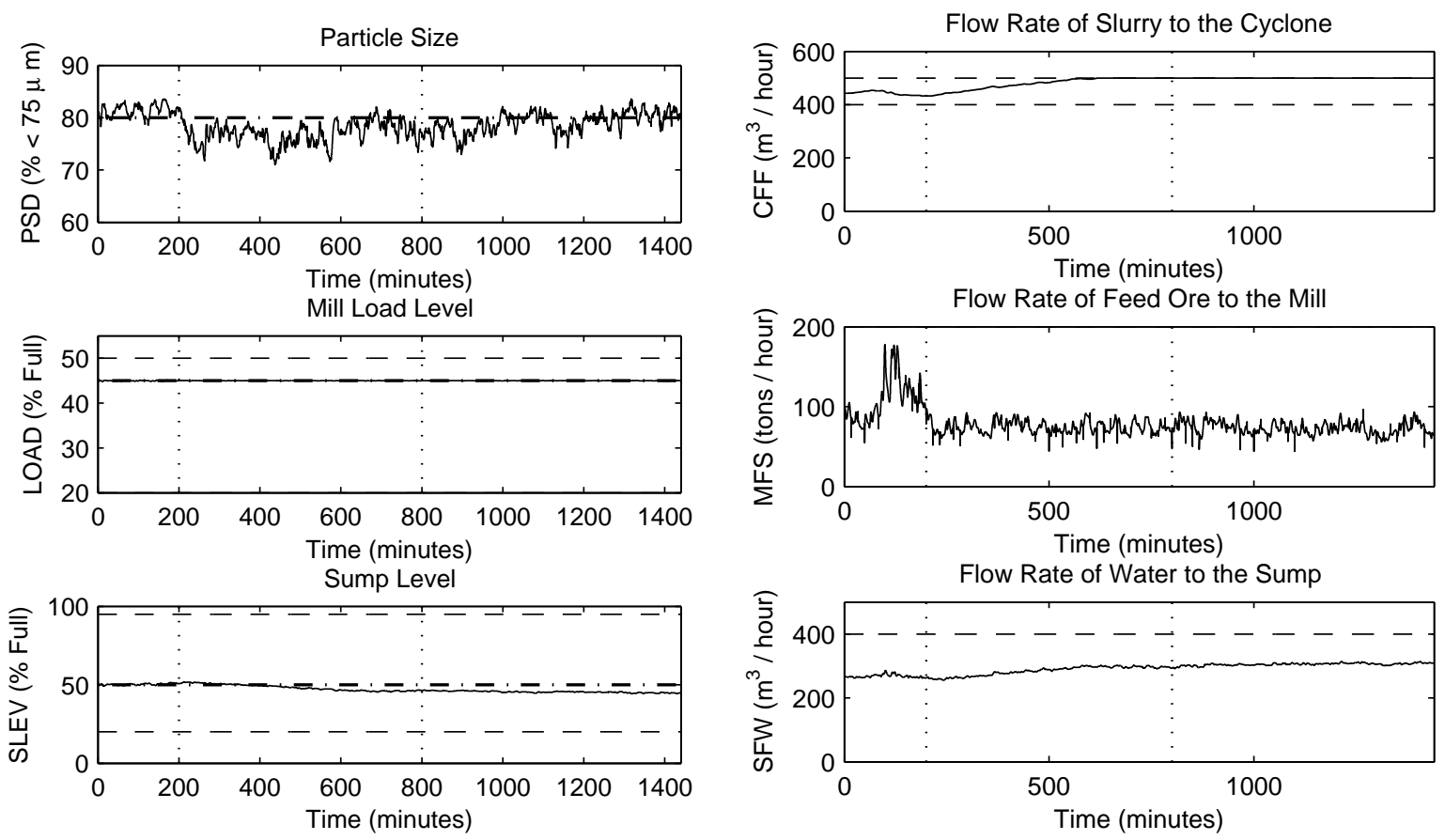

Flow Rate of Water to the Sump

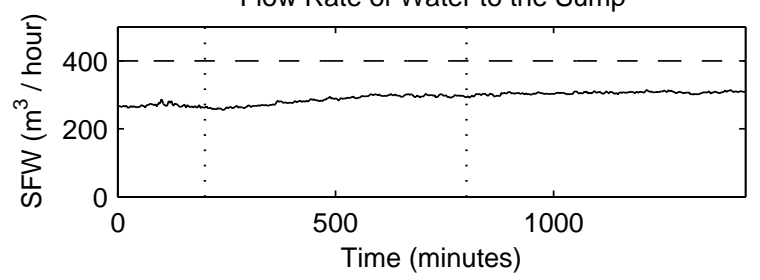

(a) CVs

(b) MVs

Figure 7.13: CVs and MVs with persistent and stationary centred disturbances for the decentralised controller

Table 7.9: Summary of simulation results with persistent and stationary centred disturbances under the decentralised controller

\begin{tabular}{ccc}
\hline Controlled variable & $\mu_{y_{i}}$ & $\sigma_{y_{i}}$ \\
\hline PSD $(\%<75 \mu \mathrm{m})$ & 78.35 & 2.67 \\
LOAD $(\%$ full $)$ & 45.00 & 0.06 \\
SLEV $(\%$ full $)$ & 48.93 & 1.64 \\
\hline
\end{tabular}




\subsubsection{Motivation for using a nonlinear controller}

Despite the considerable improvement in system performance compared to the SIMC PID controllers, the performance of the decentralised controller is still not satisfactory (ideally the PSD will stay at setpoint even with large disturbances).

Model inaccuracy is regarded as the primary sources for the 'not-very-good' performance. The mill, sump and cyclone modules are all highly nonlinear. The nonlinear static cyclone model reduces to a constant gain when linearised and reduce the usefulness of the model to a very small region around the operating point. With some disturbances that may generally happen in a ROM ore milling circuit, the circuit may move to a new operating point and will probably move outside the valid region for the linear cyclone model, while the nonlinear model will still be valid. Similar arguments holds for the mill and sump as well.

There is considerable modelling error between the nonlinear model and linear model, as shown in Section 7.2.3. In order to account for the adverse impact of the modelling error on the controlled circuit's performance, a nonlinear controller will be considered, which is intended to provide better performance. The selection of the nonlinear controller structure will be given in the following section.

\subsection{Framework step 2: Advanced controller design}

Step two in the framework discussed in Chapter 3 is the design of the new control system, as seen in Fig. 3.1. Various control design technologies can be used here. In this section the control technologies used in grinding mill circuits are described briefly. NMPC, which is selected as the advanced controller, is described in Section 7.4.2.

\subsubsection{Control technologies used in milling circuits}

Control techniques used in grinding circuits are investigated in a survey study (Wei and Craig, 2009c) and the results are shown in Fig. 7.14. The majority of the respondents use PID control (63\%). Multivariable control and expert system-based control are used less frequently but more often than other control technologies.

In support of the survey results, it is stated in Edwards et al. (2002) that the most common controller is of the PID type, which is confirmed by Ivezić and Petrović (2003), who declare that more than half of all industrial milling circuit controllers are of the PID type. There are several reasons for the domination of PID controllers. Firstly they are relatively easy to understand and implement. Higher levels of skills, which are often in short supply for the installation and maintenance of advanced controllers, are not required for PID controllers. Another barrier to the commissioning of advanced control is that plants are often reluctant to permit advanced control vendors to monitor their processes remotely, which could offset the shortage of skilled manpower. PID control, as applied to grinding circuits, is discussed in Edwards et al. (2002), Flament et al. (1997), Desbiens et al. (1997a) and Pomerleau et al. (2000). 


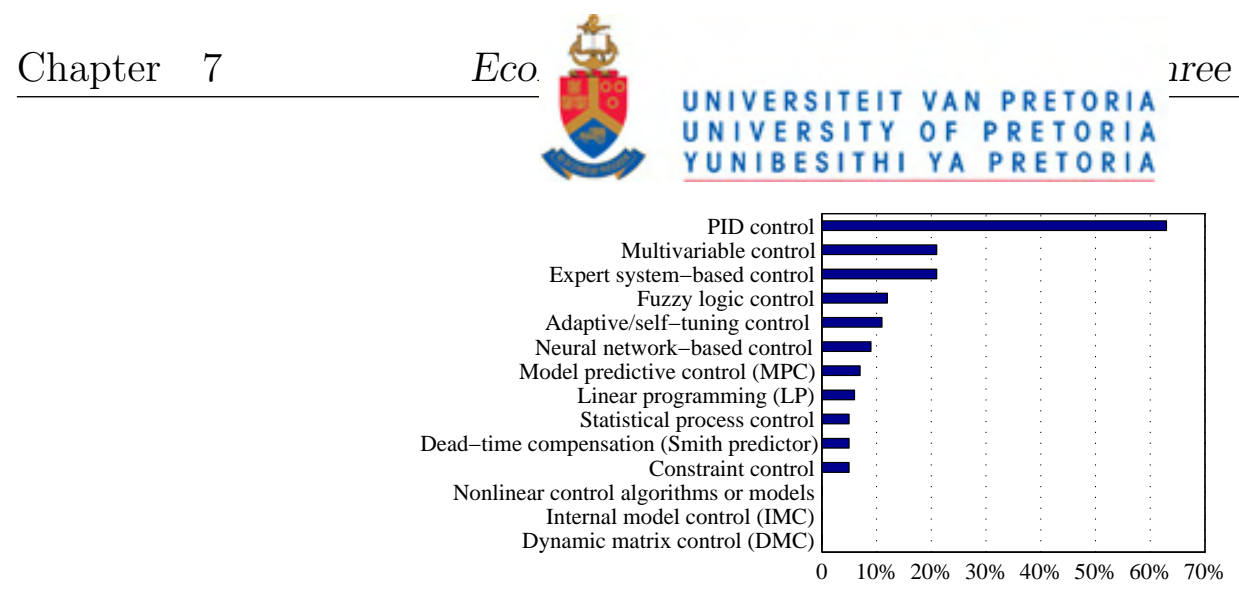

Figure 7.14: Control technologies used in milling circuits

Multivariable control is discussed in Pomerleau et al. (2000), Hulbert et al. (1990) and Craig and Macleod (1996). Numerous applications have indicated that model-based expert system controllers are useful when performing optimised supervisory control of natively complicated mineral processes (Samskog et al., 1996). Expert system-based control is discussed in Sotelo et al. (1997), Samskog et al. (1996) and Lo et al. (1996).

Fuzzy logic control is discussed in van Dyk et al. (2000) and adaptive control is investigated in Dubé and Hodouin (1989), Desbiens et al. (1997b) and Najim et al. (1995). Neural networkbased control is examined in Stange and McInnes (1995) and Flament et al. (1993) and deadtime compensation is discussed in Anderson et al. (1996).

MPC, which is the advanced control method of choice in process industries as a whole, is becoming increasingly popular for milling circuit control (Muller and de Vaal, 2000; Ramasamy et al., 2005; Chen et al., 2007; Coetzee and Craig, 2007). MPC has shown better performance in terms of setpoint tracking and the rejection of load disturbances (Freeman et al., 1994). Muller and de Vaal (2000) realise a model predictive controller for a milling circuit through simulation and results indicate that the controller is successful in controlling the particle size and in maximizing throughput.

In this chapter, an NMPC is selected as the advanced controller in view of the aforementioned popularity. Another reason is that it is also intended to provide better disturbance rejection performance due to the general advantage of MPC, handling constraints, and fairly accurate prediction of the process behaviour based on the nonlinear model. The design of the controller is summarised below.

\subsubsection{Nonlinear model predictive controller design}

The NMPC controller designed in Coetzee et al. (2008) is selected as the advanced controller. NMPC uses a nonlinear plant model to predict the behaviour of the process and derive optimal control laws in terms of a given objective function. NMPC arises from nonlinear optimal control over a time interval into the future $t \in\left[t_{k}, t_{k}+T\right]$. Only the first control move is implemented and a new measurement is obtained. The nonlinear optimal control is then recalculated for the new time interval $\left[t_{k+1}, t_{k+1}+T\right]$.

The nonlinear optimal control problem calculates a control law $u$ such that it minimises a scalar 
objective function

$$
\begin{array}{ll}
\min _{x, u} & \phi_{c}(x, u) \\
\text { s.t. } & \dot{x}(t)=f_{c}(x(t), u(t), \tilde{p}) \\
& \theta_{c}(x, u) \leq 0 \\
& t_{0} \triangleq 0, x_{0} \triangleq x\left(t_{0}\right) \\
& t_{f} \triangleq T, x_{f} \triangleq x\left(t_{f}\right), \psi\left(x_{f}\right)=0
\end{array}
$$

where $x: \mathbb{R} \rightarrow \mathbb{R}^{n_{x}}$ is the state trajectory, $u: \mathbb{R} \rightarrow \mathbb{R}^{n_{u}}$ is the control trajectory, $x(t) \in \mathbb{R}^{n_{x}}$ is the state vector, $\dot{x}(t) \in \mathbb{R}^{n_{x}}$ is the state sensitivity to time, $u(t) \in \mathbb{R}^{n_{u}}$ is the control vector, $x_{f} \in \mathbb{R}^{n_{x}}$ is the terminal state vector, $(x, u) \mapsto \phi_{c}(x, u)$ is a scalar performance function, $(x, u) \mapsto \theta_{c}(x, u)$ is the inequality constraints function, $\psi: \mathbb{R}^{n_{x}} \rightarrow \mathbb{R}$ is the terminal constraint function, $\tilde{p} \in \mathbb{R}^{n_{p}}$ is the nominal parameter vector and $f_{c}: \mathbb{R}^{n_{x}} \times \mathbb{R}^{n_{u}} \times \mathbb{R}^{n_{p}} \rightarrow \mathbb{R}^{n_{x}}$ is a vector of ordinary differential equations describing the dynamics of the plant.

The nonlinear optimal control problem is cast as a nonlinear parameter optimisation problem (Hull, 1997), which requires a continuous system model to be discretised. The discretisation is achieved by splitting the prediction horizon $[0, T]$ into $N$ discrete time intervals named nodes $t_{0} \triangleq 0<t_{1}<t_{2} \cdots<t_{k}<\cdots<t_{N-1}<t_{N} \triangleq T$ with the sampling time of $\tau_{s} \triangleq t_{k+1}-t_{k}$. The functions of time $x(\cdot)$ and $u(\cdot)$ are replaced by their values at the nodes $x_{k} \in \mathbb{R}^{n_{x}}$ and $u_{k} \in \mathbb{R}^{n_{u}}$ for $k=0, \cdots, N-1$. The nonlinear optimal control problem can now be formulated as the following nonlinear parameter optimisation problem

$$
\min \phi(\mathbf{s}, \mathbf{q})
$$

where the objective function is given by

$$
\phi(\mathbf{s}, \mathbf{q}) \triangleq \sum_{i=0}^{N-1} L_{i}\left(s_{i}, q_{i}\right)+E\left(s_{N}\right)
$$

where $\phi(\mathbf{s}, \mathbf{q}) \in \mathbb{R}$ and $\mathbf{s} \triangleq\left(s_{0}, \cdots, s_{N}\right)$ is the state sequence (for all $s_{i} \in \mathbb{R}^{n}, i=0, \cdots, N$ the estimated state parameters) and $\mathbf{q} \triangleq\left(q_{0}, \cdots, q_{N-1}\right)$ is the control sequence (for all $q_{i} \in \mathbb{R}^{m}, i=$ $0, \cdots, N-1$ the control parameters) to be optimised in the nonlinear optimisation problem (Diehl et al., 2005). The optimisation is subject to the equality constraints

$$
\begin{aligned}
x_{0}-s_{0} & =0 \\
f_{i}\left(s_{i}, q_{i}\right)-s_{i+1} & =0, i=0, \cdots, N-1,
\end{aligned}
$$

and the inequality constraints

$$
\begin{aligned}
\theta_{i}\left(s_{i}, q_{i}\right) & \leq 0, i=0, \cdots, N-1 \\
\theta_{N}\left(s_{N}\right) & \leq 0
\end{aligned}
$$

where $\theta_{i}\left(s_{i}, q_{i}\right) \in \mathbb{R}^{c}$.

The time-domain nonlinear model described in Section 7.2.1 and operating constraints and operating point described in Section 7.2.2 are used for the implementation of the NMPC controller. 
The scalar interval objective function $L_{i}\left(s_{i}, q_{i}\right)$ in this control issue is

$$
L_{i}\left(s_{i}, q_{i}\right) \triangleq\left[\begin{array}{c}
P S D \\
\text { LOAD } \\
\text { SLEV } \\
\text { THRUGHPUT } \\
\text { Rheology } \\
\text { MillPower }
\end{array}\right]^{T} Q\left[\begin{array}{c}
P S D \\
\text { LOAD } \\
\text { SLEV } \\
\text { THROUGHPUT } \\
\text { Rheology } \\
\text { MillPower }
\end{array}\right]+\left[\begin{array}{c}
\Delta C F F \\
\Delta M F S \\
\Delta S F W \\
\Delta M I W \\
\Delta \text { Balls }
\end{array}\right]^{T} R\left[\begin{array}{c}
\Delta C F F \\
\Delta M F S \\
\Delta S F W \\
\Delta M I W \\
\Delta \text { Balls }
\end{array}\right]
$$

where $Q$ and $R$ are diagonal weighting matrices that have an impact on the states and the controls respectively, as shown below:

$$
Q=\left[\begin{array}{cccccc}
100 & 0 & 0 & 0 & 0 & 0 \\
0 & 100 & 0 & 0 & 0 & 0 \\
0 & 0 & 1 & 0 & 0 & 0 \\
0 & 0 & 0 & 0 & 0 & 0 \\
0 & 0 & 0 & 0 & 0 & 0 \\
0 & 0 & 0 & 0 & 0 & 0
\end{array}\right]
$$

and $R$ is given by

$$
R=\left[\begin{array}{ccccc}
0.1 & 0 & 0 & 0 & 0 \\
0 & 0.1 & 0 & 0 & 0 \\
0 & 0 & 0.1 & 0 & 0 \\
0 & 0 & 0 & 0.1 & 0 \\
0 & 0 & 0 & 0 & 0.1
\end{array}\right]
$$

For the terminal objective function $E\left(s_{N}\right)\left(\triangleq s_{N}^{T} P s_{N}\right)$, the $P$ that is equivalent to $Q$ is used. The tuning of the weights mostly depends on trial and error, mixed with control priority. It can be seen that the control moves are on average weighted less than the setpoint values to ensure that the setpoints get higher priority than the control moves. Each setpoint is then weighted according to priority. For example, the PSD and the THROUGHPUT are trade-offs. If they are weighted the same, neither of them will reach its setpoint. If the PSD is weighted higher than the THROUGHPUT, then the PSD will operate closer to its setpoint and THROUGHPUT further from its setpoint. If the setpoints are independent, the relative weighting between setpoint variables does not matter. The relative weighting of the setpoint variables with the control moves (MVs) do still matter, though. If the MVs are weighted higher than the setpoint variables, then the setpoints will not be reached, as the controller attempts to minimise MV deviation. If the change in MV is however weighted, then the setpoints will still be reached, just more slowly, and therefore the relative weighting between the setpoint variables and MVs are not that important. The actual values are not actually important; just the relative difference in values is of importance. If the variables are not scaled, then the weightings have to be scaled to compensate for this. The NMPC controller scales the values and weightings, which therefore do not have to be adjusted.

The scaling on the MVs, which is done in the circuit modelling part and related to the weights selection here, is to ensure that the scaled range for all of them is the same, e.g. all the MVs are scaled to change between 0 and 1 . The MVs are typically scaled to their constraint ranges (e.g. MFS unscaled range [0-200] tons/h is scaled into [0-1]). This just means that all the MVs will be penalised the same amount in the objective function if they are at their constraints. If the MVs were not scaled, and $R$ has equal entries for all of them, an MV with a small range 
will be penalised less that an MV with a big range. For instance, MV1 has a range [-1 1$]$ and MV2 has a range [-20 20]. In the objective function, MV2 will have a penalty of $400\left(M V 2^{2}\right)$ at its constraint, while MV1 will have a penalty of $1\left(M V 1^{2}\right)$. The controller might then change MV1 more than MV2 because it is less 'expensive'.

There is a rule of thumb that the prediction horizon should be at least 2.5 times the slowest time constant. This is not practical with the NMPC controller, as it would take too long to calculate. Specifically, the slowest time constant is 1 hour and would require a prediction horizon of $2.5^{*} 360$ at a 10 -second sampling time. A longer prediction horizon allows more of the dynamics to be captured, especially the time delays, and improves stability, but also makes the calculation slower. The prediction horizon length is adjusted to find the best trade-off between performance and calculation time. The control horizon and prediction horizon are both selected to be 6 . The sampling time of 10 seconds is used. IPOPT (Kawajir et al., 2006) is used to solve the large-scale sparse nonlinear optimisation problem. In addition, CPPAD (Lougee-Heimer, 2003) is used to perform the numeric differentiation associated with Eq. (7.70).

\subsubsection{Performance of the nonlinear model predictive controller}

The designed NMPC controller is used in simulations to control the milling circuit under the same disturbance scenarios used in Section 7.3.2. The simulation results under stationary centred disturbances are given in Fig. 7.15. It can be seen that all CVs are controlled well and all MVs do not saturate.

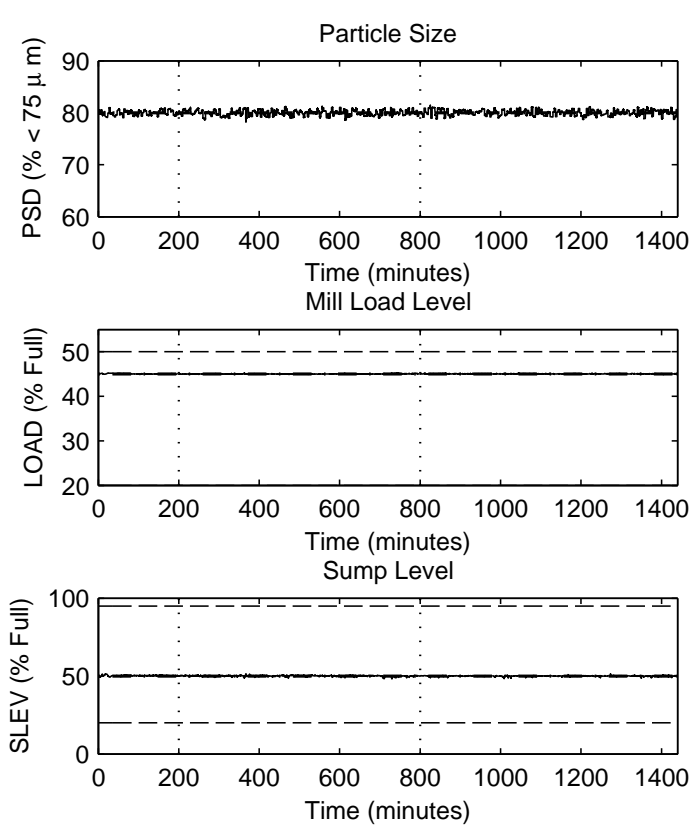

(a) $\mathrm{CVs}$

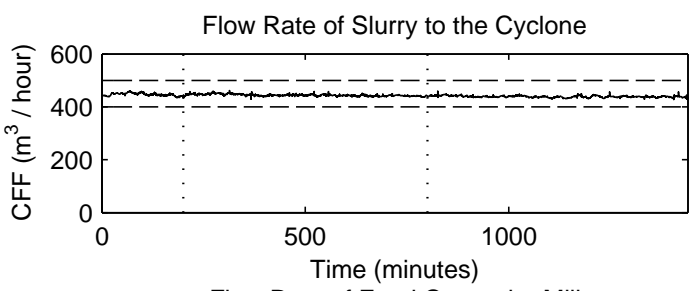

Flow Rate of Feed Ore to the Mill

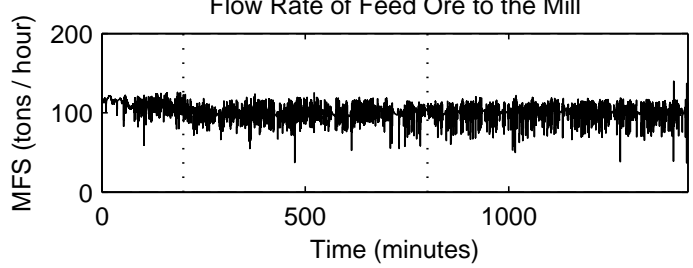

Flow Rate of Water to the Sump

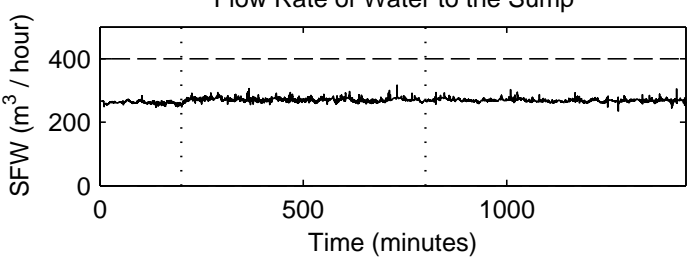

(b) MVs

Figure 7.15: CVs and MVs of one-day operation for the NMPC controller under stationary centred disturbances

The results associated with persistent plus stationary disturbances are given in Fig. 7.16 and the statistics in Table 7.10. It can be seen that NMPC performs much better than the PID controllers with respect to disturbance rejection. The average recovery (EPI) for one day's 
operation for the NMPC controller can then be calculated, as done in Section 7.3.3, using Eq. (7.55), being 70.66\%. The relevant recovered mineral value is $70.66 \mathrm{k} \%$, using Eq. (7.56).
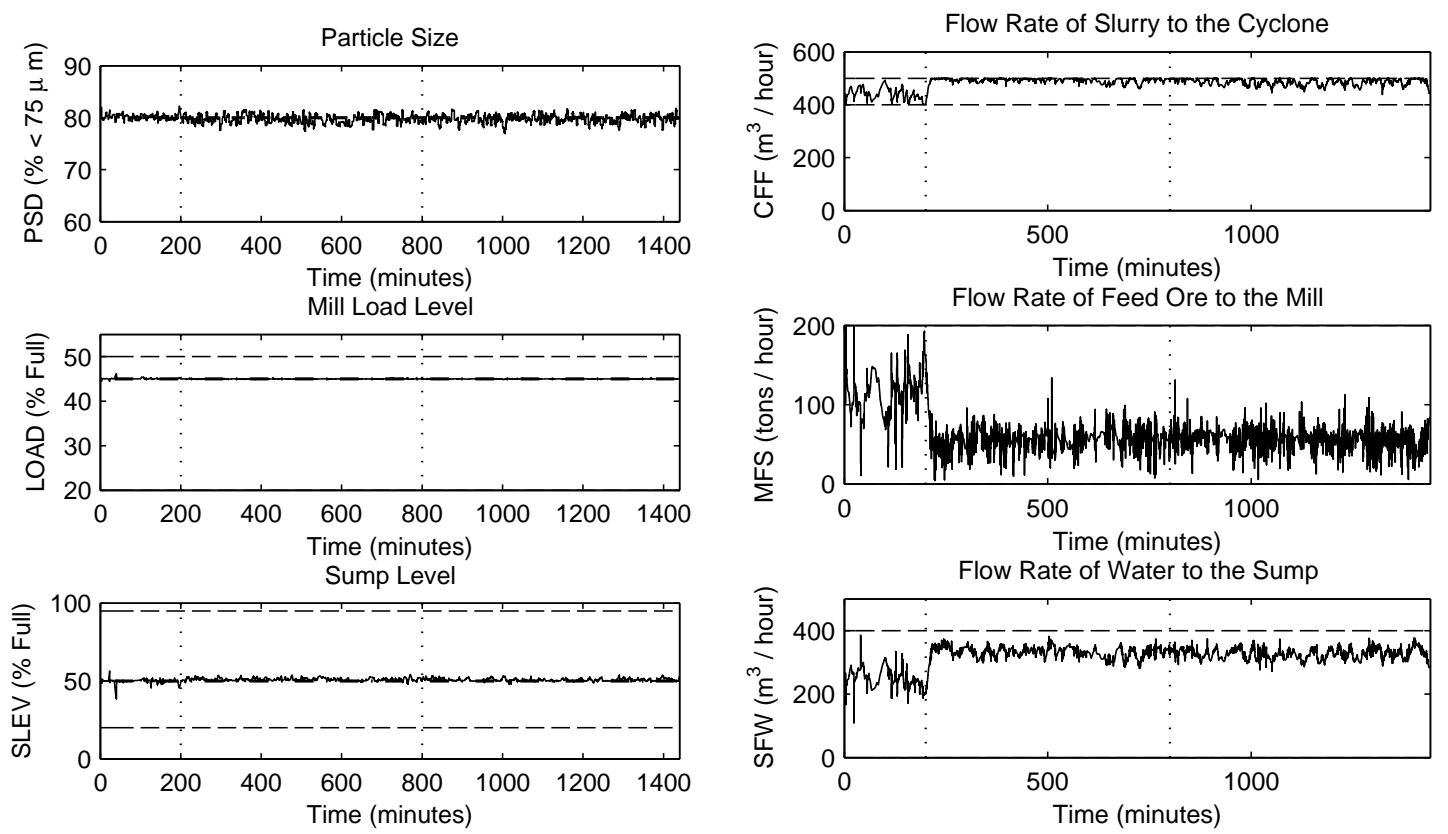

(a) CVs

(b) MVs

Figure 7.16: CVs and MVs of one-day operation for the NMPC controller under persistent plus stationary disturbances

Table 7.10: Statistical data of CVs for the NMPC controller under stationary and persistent disturbances

\begin{tabular}{ccc}
\hline Controlled variable & $\mu_{y_{i}}$ & $\sigma_{y_{i}}$ \\
\hline PSD $(\%<75 \mu \mathrm{m})$ & 79.82 & 0.81 \\
LOAD $(\%$ full $)$ & 44.99 & 0.07 \\
SLEV $(\%$ full $)$ & 50.77 & 1.06 \\
\hline
\end{tabular}

To summarise the performance of the three control systems, the system responses of one-day operation associated with the SIMC PID controllers, the decentralised controller and the NMPC controller under two different disturbance scenarios are shown together to facilitate comparison. The time trends of CVs are given in Fig. 7.17 and relevant statistics in Table 7.11. It can be seen that under stationary disturbances, the NMPC controller does not provide a considerable advantage. However, when persistent disturbances occur in the circuit, the NMPC is better at rejecting disturbances.

\subsubsection{Analysis of the origin of the advantage of the nonlinear con- troller}

One reason for the NMPC being better is that it has access to MIW and MFB that the PID controllers do not have. Even if the PID controllers do have access to MIW and MFS, they should be designed to control PSD somehow and accordingly have three MVs controlling one $\mathrm{CV}$. The NMPC can, therefore, exploit the multivariate nature of the process within the 


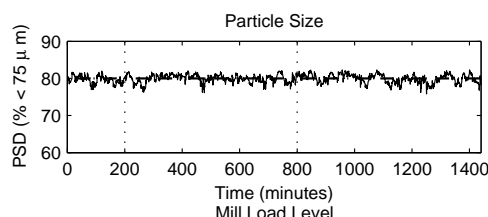
Mill Load Leve
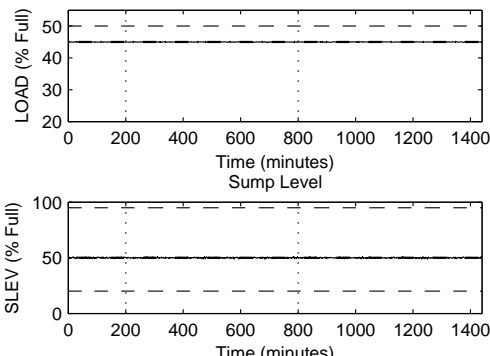

(a) CVs of SIMC PID with stationary disturbances
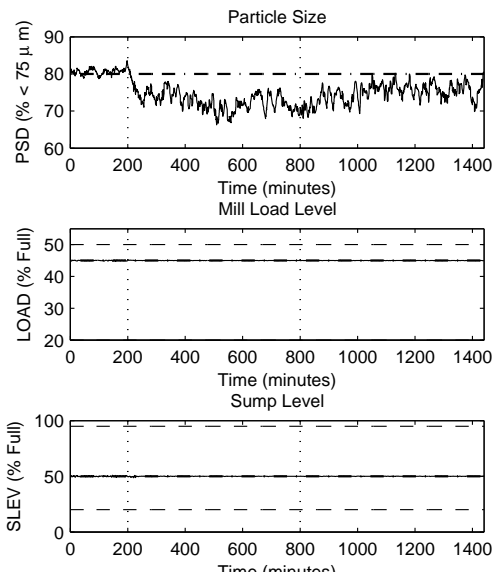

(d) CVs of SIMC PID with persistent and stationary disturbances
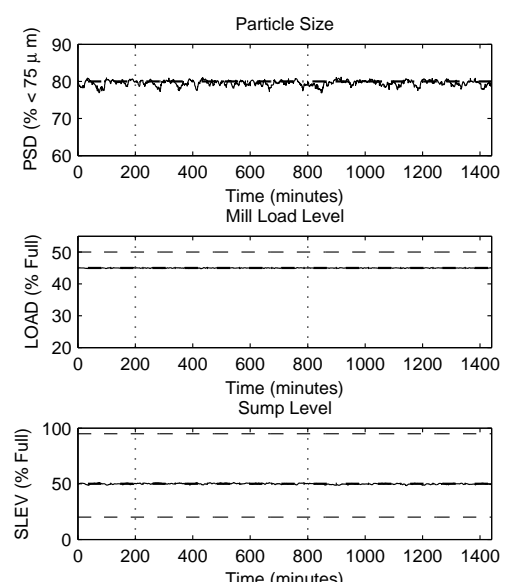

(b) CVs of the decentralised controller with stationary disturbances
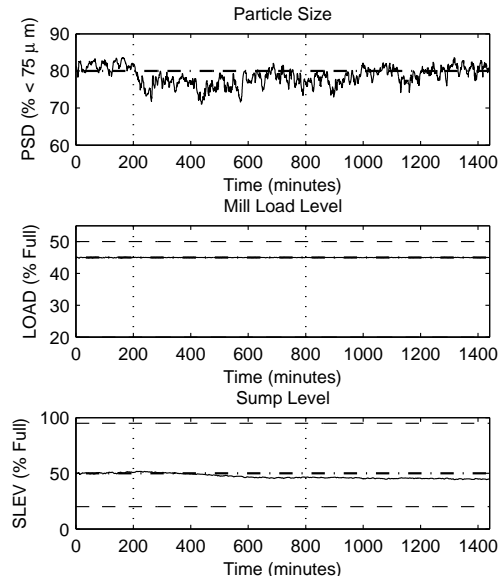

(e) CVs of the decentralised controller with persistent and stationary disturbances
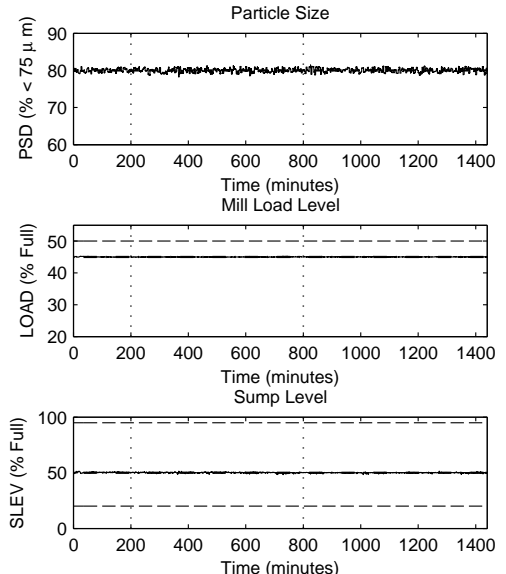

(c) CVs of NMPC with stationary disturbances
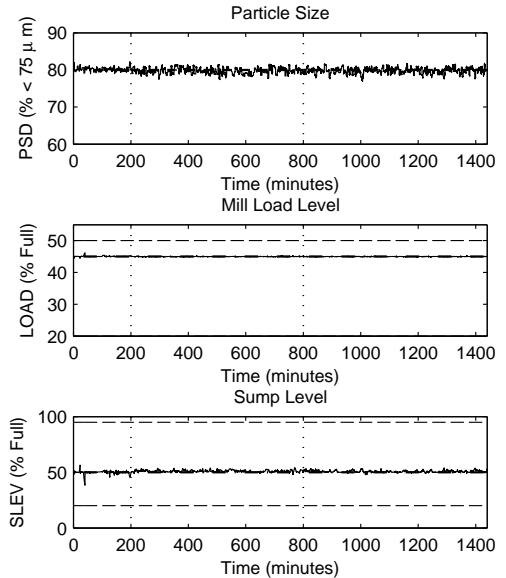

(f) CVs of NMPC with persistent and stationary disturbances

Figure 7.17: CVs of three controllers under two disturbance scenarios

Table 7.11: Summary of simulation results of the SIMC PID controllers, decentralised controller and NMPC controller under two disturbance scenarios: Scenario 1 refers to stationary centred disturbances and Scenario 2 refers to stationary and persistent disturbances.

\begin{tabular}{|c|c|c|c|c|c|c|c|}
\hline & & SIMC & PID & Dec. cc & troller & NM & \\
\hline \multirow{4}{*}{ Scenario 1} & $\mathrm{CV}$ & $\mu_{y_{i}}$ & $\sigma_{y_{i}}$ & $\mu_{y_{i}}$ & $\sigma_{y_{i}}$ & $\mu_{y_{i}}$ & $\sigma_{y_{i}}$ \\
\hline & $\operatorname{PSD}(\%<75 \mu \mathrm{m})$ & 79.83 & 0.82 & 79.78 & 0.61 & 79.97 & 0.57 \\
\hline & LOAD (\% full) & 45.00 & 0.04 & 45.00 & 0.06 & 45.00 & 0.04 \\
\hline & SLEV (\% full) & 49.99 & 0.27 & 51.04 & 0.37 & 51.02 & 0.25 \\
\hline \multirow{4}{*}{ Scenario 2} & CV & $\mu_{y_{i}}$ & $\sigma_{y_{i}}$ & $\mu_{y_{i}}$ & $\sigma_{y_{i}}$ & $\mu_{y_{i}}$ & $\sigma_{y_{i}}$ \\
\hline & $\mathrm{PSD}(\%<75 \mu \mathrm{m})$ & 75.05 & 3.94 & 78.35 & 2.67 & 79.82 & 0.81 \\
\hline & LOAD (\% full) & 45.00 & 0.04 & 45.00 & 0.06 & 44.99 & 0.07 \\
\hline & SLEV (\% full) & 49.99 & 0.17 & 48.93 & 1.64 & 50.77 & 1.06 \\
\hline
\end{tabular}


constraints provided, whereas the PID controllers cannot intelligently use the MIW, MFB, CFF and SFW to control PSD, while keeping SLEV at setpoint.

The NMPC has the knowledge of the cyclone curve that describes the effect of feed density and cycle feed flow rate on the final product. The PID controllers can typically only control density or flow, but not both. The NMPC can manipulate the MIW, SFW and CFF to find the best combination of the three MVs in order to optimise density and flow to the cyclone within the constraints to produce the required product particle size. Because one PID controls the SLEV and the other PID controls flow, the PID that controls flow will try to change the flow to get the required PSD, but the other loop will compensate for the extra flow on the SLEV by increasing the SFW, which in turn affects density in the sump. The density will become lower as the CFF increases, because of the increase in the SFW, but the CFF cannot increase enough to lower density sufficiently. The NMPC further uses MIW to reduce the density of the slurry discharging from the mill to maintain PSD.

If the NMPC is restricted to the same number of MVs, it could also change SLEV to change the cyclone feed density that a PID would not be able to do, because the PID would always want to follow its setpoint. The NMPC can be tuned to make SLEV less important than PSD that will cause the NMPC to increase SLEV in order to maintain PSD at setpoint.

\subsection{Framework step 3: Estimation of performance and initial economic assessment}

In step 3 of the framework, the functional performance of advanced control is estimated first and then it is translated into economic performance, which will be compared to the economic performance of the base case to see how much the improvement is and whether the advanced controller is worth commissioning. If the answer is negative, the procedure is to re-design the advanced controller. Otherwise, the controller will be commissioned.

When using only the stationary centred disturbance scenario, a feasible conclusion after step 3 might be not to implement the NMPC controller. The purpose of this chapter, however, is to illustrate the EPA process, and hence the persistent plus stationary scenario will be used in what follows.

\subsubsection{Estimate advanced process controller's performance}

In order to justify the investment in the implementation of the selected control technology, an estimate of the potential benefit that may result from the technology is required, as described in step three of the framework. In step three, the functional performance of the new controller needs to be estimated with two sub-objectives. The first is to estimate the reduction in the variance of process variables. The second is to determine the new setpoints for process variables with the new controller, which can be calculated based on the reduction in the variance and some optimisation strategy (Latour, 1996; Bauer et al., 2007).

Estimating the performance of an advanced controller usually results in estimating the resulting 
reduction in the standard deviation of CVs. When the optimal operating point is near a constraint, which is often the case, the setpoint can be moved closer to the optimal point when the standard deviation of a $\mathrm{CV}$ is reduced.

Considering that the PID controller works well with respect to the LOAD and SLEV, the focus here is on the PSD. $50 \%$ is selected as the estimate for the reduction in $\sigma$, i.e. the NMPC will reduce the $\sigma_{P S D}$ by $50 \%$ as compared to PID control (from 3.94 to 1.97), which is more conservative than the results of Section 7.4.3 suggest. The operating point of the PSD for the NMPC controller is assumed as $80 \%$ owing to its improved performance disturbance rejection shown in Section 7.4.3.

\subsubsection{Initial economic assessment of the two controllers}

In this section, the estimate of the EPI associated with the NMPC controller is undertaken by using the estimated operating point and $\sigma$ obtained in Section 7.5.1 and Eq. (7.55) to transfer functional performance into economic performance. The EPI of the new controller can then be compared to the EPI of the base case to judge whether there is a significant improvement in the system's economic performance. Using the minimum variance, the upper limit of the potential achievable performance improvement was derived in Section 7.3.4. The EPI of the new controller can also be compared to this upper limit to judge whether the improvement is satisfactory in terms of what can be achieved. If the EPI improvement is not acceptable, then the controller design of step two is repeated. Otherwise, the controller that was designed will be implemented.

It is assumed that the NMPC will perform no worse than the PID controllers in terms of LOAD and SLEV control, therefore, these two variables will remain within constraints. Based on the estimate that the NMPC will reduce the PSD $\sigma$ by $50 \%$ at the operating point for the PSD of $80 \%$, the average recovery (EPI) for the mineral is derived as $70.65 \%$ using Eq. (7.55). The value of the mineral recovered is $(70.65 \mathrm{k}) \%$ using Eq. (7.56), whereas the recovered mineral value of the base case is $(69.61 \mathrm{k}) \%$. It can be seen that the recovered mineral value under NMPC control will then be $(1.04 \mathrm{k}) \%$ more than under PID control. This may be an economically significant improvement, depending on the mineral processed.

\subsection{Framework step 4: Implementation of the advanced controller}

Assume now that the NMPC controller is implemented because of the significant potential recovery improvement. The main contributors to the costs of implementing a grinding control system are investigated in Wei and Craig (2009c) and the results are shown in Fig. 7.18. The major contributors are control hardware, consultant manpower cost and the cost of the technology. These costs would form part of $\Delta C$ in Eq. (6.5). This is in contrast to results reported in Bauer and Craig (2008) for process industries as a whole, where manpower cost is dominant and control hardware plays a lesser role. In the next section, the advantage of the NMPC controller will be investigated further using a statistical experiment, as a simple PID/NMPC comparison is not sufficient (Craig and Koch, 2003). 


Chapter $7 \quad$ Eco.

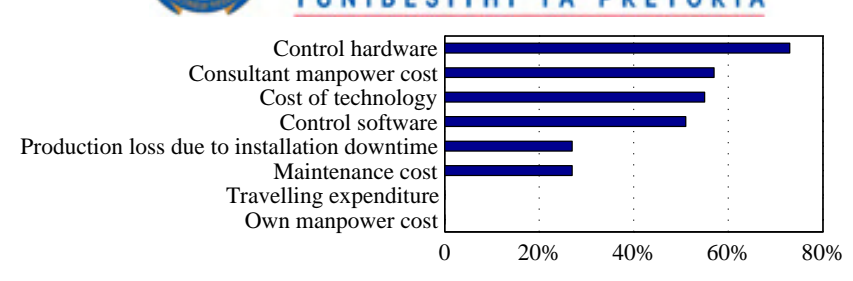

Figure 7.18: Cost types of a process control system

\subsection{Framework step 5: Calculation of performance and success assessment}

\subsubsection{Statistical comparison of two controllers}

In step five of the framework, the first task is to evaluate the functional performance of the new controller after implementation. It is convenient to do since the operating data are now available and the PDFs of the time series of the process variables can be derived.

\subsubsection{Motivation for the statistical experiment}

Although the results of Section 7.5.2 may indicate that some economic benefits resulting from improved control are possible, a single comparative test is not sufficient. It might happen that the experimental conditions favour NMPC, causing misleading results (Craig and Henning, 2000). The advanced controller might also have been tuned for a particular set of disturbances and it might not work well once other disturbances are introduced. In order to improve the accuracy of the comparison between the NMPC and PID controllers, a series of tests needs to be undertaken using experiment design techniques (Chatfield, 1983; Craig and Koch, 2003).

\subsubsection{Modelling of disturbances}

Feed ore hardness and composition changes are major disturbances that milling circuit controllers have to contend with. They are varied in the simulations described below to reflect typical operating conditions for the milling circuit being considered.

In particular, ore hardness and composition changes are modelled as both random and step disturbances. Hardness has a direct impact on the energy needed for grinding and ore composition refers to the rock fraction of the feed (Coetzee et al., 2008). Step disturbances simulate instances where e.g. the feed ore is switched between feeds that originate from different stockpiles. Uniform random disturbances are used to simulate natural variations in hardness and composition in the range [-80\% 80\%] where zero is the nominal value. Stockpile segregation is modelled as a slow linear drift from a value of 36.4 to $53.2(\mathrm{kWh} / \mathrm{t})$ and from 0.15 to 0.04 (dimensionless) for hardness and rock fraction respectively. 


\subsubsection{State the hypothesis}

For the purpose of this study, the performance of the PID and NMPC controlled milling circuits needs to be compared statistically. The LOAD and SLEV, controlled to stabilise the system, are controlled well and remain within the constraints for both cases. Hence, it is not necessary to compare these two variables statistically. The focus of the comparison is put on the PSD. The comparison of the mean PSD resulting from the PID and NMPC controllers can be related to a recovery difference that is economically meaningful. A trade-off relation between quality (grind) and throughput exists for the milling circuit, as shown in Fig. 7 of Bauer and Craig (2008), i.e. the improvement in average grind will be at the expense of a decrease in average throughput. The significance of the PSD comparison is that, because of the characteristics of the PF of the PSD, the closer the operating point is to the setpoint, the better recovery will be achieved, which may cause significant economic improvements. The relevant null and alternative hypotheses are:

$H_{0}: \mu_{P S D_{P I D}}=\mu_{P S D_{N M P C}}$

$H_{1}: \mu_{P S D_{P I D}}<\mu_{P S D_{N M P C}}$

The NMPC controller will then be regarded as effective if $H_{0}$ can be rejected with a sufficient degree of significance. The experiment duration required to detect performance differences between two controllers depends on the degree of precision required, the amount of variability in the experimental material, and the available resources to conduct the experiment. In general, a higher significance level leads to a shorter experiment. As plant experiments are very expensive to conduct, and given the large variations in process variables that often occur relative to the change that the hypothesis test is testing for, the significance level is selected as $\alpha=0.15$ for the purpose of this experiment. See Craig and Koch (2003), for a more detailed explanation.

It is also necessary to test whether there is a significant difference in the throughput rate (THR), which is also important practically. The null and alternative hypotheses are:

$H_{0}: \mu_{T H R_{P I D}}=\mu_{T H R_{N M P C}}$

$H_{1}: \mu_{T H R_{P I D}}>\mu_{T H R_{N M P C}}$

The THR with the NMPC controller will then be regarded as statistically less than that for PID control if $H_{0}$ can be rejected with a sufficient degree of significance. The significance level is also selected as $\alpha=0.15$.

The recovery $(R)$ difference for the two controllers also needs to be statistically tested to see whether there is a considerable improvement. The null and alternative hypotheses are:

$H_{0}: \mu_{R_{P I D}}=\mu_{R_{N M P C}}$

$H_{1}: \mu_{R_{P I D}}<\mu_{R_{N M P C}}$

Similarly, the recovery under NMPC will be regarded as significantly improved if $H_{0}$ can be rejected with a sufficient degree of significance. The significance level is also selected as $\alpha=0.15$.

\subsubsection{Experimental design}

Statistical techniques are available to assess the performance of processes operating under uncertainty. However, the use of these techniques in some process control industries is still relatively limited, compared to, e.g. the biological and social sciences (Craig and Koch, 2003). The performance of two controllers is regularly compared by simply switching between old and new controllers in a 'month-on, month-off' manner (Bauer and Craig, 2006). Plant improvement trials are therefore often inappropriately designed, and result in either no conclusion where a 
useful conclusion could be reached, or an incorrect conclusion (Napier-Munn, 1995).

Comparative experimental techniques can be classified approximately in terms of replication, blocking and randomisation (Chatfield, 1983). Most comparative experimental techniques either take one of these divisions or a combination of two or three.

In this study, a complete randomised design is used to perform the experiment. The disturbances of the hardness and composition vary over time and randomisation is used to ensure that the test data will be uncorrelated with these time trends. Blocking is not used, as the subdivision of the experiment into smaller time intervals will not reduce the variations of the data. If no considerable difference occurs, blocking will probably affect the accuracy of the experiment results adversely (Fisher and Yates, 1953). It is usually regarded as good practice to keep an experiment as simple as possible (Oosthuizen, 2001).

The selection of the duration for each test is based on the characteristics of the system and the test needs to be significantly longer than the longest time constant of the process. The duration is recommended to vary between one and three days in Craig and Henning (2000). In this study, one day is selected for the running period for each test, such that the milling circuit can reach steady state under varying disturbances. The number of observations (in the form of simulations) is selected as 200 and each is 24 hours long. A pseudo-random binary algorithm was used to determine which controller will be used when; the NMPC controller is used 103 times and the PID 97 times.

\subsubsection{Data generation and hypothesis testing}

7.7.1.5.1 Data generation The simulations are conducted using the disturbances described in Section 7.7.1.2 and the experimental design described in Section 7.7.1.4. For the results of each day-long simulation, the average and $\sigma$ of the PSD, LOAD and SLEV are derived. The results of interest are summarised in Table 7.12. The average PSD with the NMPC controller is improved by $4.62 \%$, compared to PID control. Note that the PSD setpoint for each controller is $80 \%$ passing $75 \mu \mathrm{m}$.

Table 7.12: Summary of simulation results of statistical experiments

\begin{tabular}{|c|c|c|c|c|c|}
\hline \multirow{2}{*}{ Process variable } & \multirow{2}{*}{ Setpoint } & \multicolumn{2}{|c|}{ Mean } & \multicolumn{2}{|c|}{$\sigma$} \\
\cline { 3 - 6 } & & NMPC & PID & NMPC & PID \\
\hline PSD & $80(\%<75 \mu \mathrm{m})$ & 79.87 & 75.25 & 0.21 & 3.95 \\
\hline Throughput rate & & 71.88 & 72.55 & 4.87 & 5.24 \\
\hline Recovery & & 70.66 & 69.60 & 0.03 & 1.01 \\
\hline
\end{tabular}

The $t$-test is used most commonly for comparative experiments. The two-sample $t$-test is used in this work, in which the means of the two sample sets are calculated and compared using standard statistical methods. The validity of the $t$-test is based on the following assumptions (Napier-Munn, 1995):

1. The data are distributed normally.

2. Each data point is a random independent sample of the population of all probable experimental results for the process considered.

3. The two sample variances are the estimates of the same population variance. 
The first assumption is accepted a priori, which holds for most plants (Thornhill and Horch, 2007). The second assumption is also satisfied, as a complete randomised experiment design is used to ensure that the data samples are unrelated to the time trends of disturbances. The third assumption needs be verified using an $F$-test, which investigates whether two standard deviations are significantly different.

7.7.1.5.2 Hypothesis test on the particle size distribution As far as the PSD is concerned, the null hypothesis for the $F$-test is $H_{0 F}: s_{P I D}^{2}=s_{N M P C}^{2}$. The alternative hypothesis is $H_{1 F}: s_{P I D}^{2}>s_{N M P C}^{2}$. The $s_{P I D}^{2} / s_{N M P C}^{2}$ follows an $F$ distribution with the degrees of freedom 96 and 102, whereas the sample size for PID and NMPC is 97 and 103 respectively. In order not to reject the $H_{0 F}$ with a significance level of 0.85 , the $s_{P I D}^{2} / s_{N M P C}^{2}$ needs to be smaller than $F_{\text {inv }}(0.85,96,102)$, that is 1.23 . However, as the $s_{P I D}^{2} / s_{N M P C}^{2}$ is 345.60 , the $H_{0 F}$ can then be rejected and the third assumption is not satisfied. The standard $t$-test can therefore not be used.

However, a robust $t$-test (Fisher and Yates, 1953) can be used, which can still provide valuable information. With this method, the pooled variance needs to be estimated based on the standard deviations of two samples, as given below:

$$
\begin{aligned}
s^{2} & =\frac{\left(n_{1}-1\right) s_{1}{ }^{2}+\left(n_{2}-1\right) s_{2}{ }^{2}}{n_{1}+n_{2}-2} \\
& =\frac{(97-1) 3.95^{2}+(103-1) 0.21^{2}}{97+103-2} \\
& =7.60
\end{aligned}
$$

where $n_{1}$ and $n_{2}$ are the sample size for the PID and NMPC control respectively, and $s_{1}$ and $s_{2}$ are the standard deviations of the PSD for PID and NMPC control respectively. The test statistic $t$ is derived as follows:

$$
\begin{aligned}
t & =\frac{\mu_{P S D_{N M P C}}-\mu_{P S D_{P I D}}}{s \sqrt{1 / n_{1}+1 / n_{2}}} \\
& =\frac{79.87-75.25}{2.76 \sqrt{1 / 97+1 / 103}} \\
& =11.86
\end{aligned}
$$

The value of the statistic $t$ for the $85 \%$ confidence level with the degree of freedom 198 is 1.04 . $H_{0}$ can therefore be rejected with $85 \%$ confidence. The NMPC therefore provides better control statistically in respect of the PSD, the improvement being $4.62 \%$.

7.7.1.5.3 Hypothesis test on the throughput The hypothesis test for the throughput rate is conducted, as for the PSD. The values of critical statistics are shown in Table 7.12. An $F$-test shows that the two throughput rate samples are from the same population, since the value of $s_{P I D}^{2} / s_{N M P C}^{2}$ is 1.02 , which is less than $F_{i n v}(0.85,96,102)(1.23)$. Then a standard $t$-test is conducted. The statistic $t$ is

$$
\begin{aligned}
t & =\frac{\mu_{T H R_{P I D}}-\mu_{T H R_{N M P C}}}{\sqrt{\sigma_{1}^{2} / n_{1}+\sigma_{2} / n_{2}}} \\
& =\frac{72.55-71.88}{\sqrt{5.24^{2} / 97+4.87^{2} / 103}} \\
& =0.94
\end{aligned}
$$


Since the value of the $t$ is smaller than the $T_{i n v}(0.85,198)$, that is 1.04 , the null hypothesis can be accepted with significance level 0.15 . Then, it can be regarded that the throughput for PID controllers is, statistically speaking, the same as that for NMPC with 0.85 confidence. The hypothesis test for recovery and recovered mineral value in downstream flotation circuits will be conducted in Section 7.7.2, as it forms part of the economic assessment.

\subsubsection{Final assessment of the nonlinear model predictive controller and proportional-integral-derivative controller}

In this section the EPA of the PID and NMPC controllers is conducted based on the statistical experiment described in Section 7.7.1. In the experiment, the NMPC controller runs for 103 times and the PID for 97 times, with each run equalling 24 hours. Data of the controlled and manipulated variables are now available for each of the 200 runs. From the data the time series and PDF of the PSD can be obtained; further, based on the assumption of Gaussianity made above, the mean and standard deviation can be obtained. Based on the PF and the PDF of the PSD, the average mineral recovery in the downstream flotation circuit for each simulation is then derived using Eq. (7.55). Subsequently, the overall average mineral recovery for NMPC can be derived by averaging the 103 average recoveries for NMPC and 97 average recoveries for PID control. The value of the mineral recovered for a given time can then be obtained based on the overall average recovery and other parameters such as head grade, throughput rate and so on for either NMPC or PID control, using Eq. (6.1).

Note that it is not good practice to obtain the overall average PSD and then to map the overall average PSD to the recovery, as this mapping only accounts for the mean information but neglects the role of the variation in the PSD. The mapping will lead to a less accurate estimate of the system's performance due to the oversimplification of the economic model (Martin et al., 1991).

\subsubsection{Hypothesis test on recovery}

The average recovery for the NMPC controller is $70.66 \%$, which is $1.06 \%$ more than the $69.60 \%$ achieved by the single-loop PID controllers. A hypothesis test is conducted to examine the statistical significance of the improvement, as conducted in Section 7.7.1.5. The standard deviation of the recovery is 0.03 and 1.01 for NMPC and PID respectively. An F-test indicates that two recovery samples are not from the same population, since the value of $s_{P I D}^{2} / s_{N M P C}^{2}$ is 1099.70, which is bigger than $F_{i n v}(0.85,96,102)(1.23)$. A robust $t$-test is then used to examine whether the difference of recovery is statistically significant. The pooled variance is estimated as

$$
\begin{aligned}
s^{2} & =\frac{\left(n_{1}-1\right) s_{1}^{2}+\left(n_{2}-1\right) s_{2}{ }^{2}}{n_{1}+n_{2}-2} \\
& =\frac{(97-1) 1.01^{2}+(103-1) 0.03^{2}}{97+103-2} \\
& =0.49
\end{aligned}
$$

where $n_{1}$ and $n_{2}$ are the sample size for PID and NMPC control respectively, and $s_{1}$ and $s_{2}$ are the standard deviations of the PSD for PID and NMPC control respectively. The test statistic 
$t$ is derived as follows:

$$
\begin{aligned}
t & =\frac{\mu_{R_{N M P C}}-\mu_{R_{P I D}}}{s \sqrt{1 / n_{1}+1 / n_{2}}} \\
& =\frac{70.66-69.60}{0.71 \sqrt{1 / 97+1 / 103}} \\
& =10.60
\end{aligned}
$$

The value of the statistic $t$ for the $85 \%$ confidence level with the degree of freedom of 198 is 1.04. $H_{0}$ can therefore be rejected with $85 \%$ confidence. The recovery for NMPC is therefore significantly more than that of the PID controllers.

\subsubsection{Multivariate economic assessment regarding throughput and particle size}

In this subsection, a multivariate economic assessment is conducted that investigates the THR and PSD jointly. In this case, the PSD reflects the production quality. There is generally a trade-off between throughput and quality as shown in Bauer and Craig (2008), as is the case here for THR and PSD. When the value of mineral recovered in the downstream flotation circuit is considered as the index of the system's economic performance, it is mainly determined by the THR and PSD given that the mineral price and head grade of the circuit's product remain consistent. Both the THR and PSD are closely related to the recovered mineral value and they have opposite impacts on the value. When the recovered mineral value is considered, these two variables need to be examined jointly. The structure of this subsection is as following. The dependence of THR and PSD will be investigated first, followed by the establishment of their JPF and JPDF. The multivariate economic performance will then be discussed.

7.7.2.2.1 Dependence of the throughput rate and particle size Scatter plots, as shown in Fig. 7.19, are used to investigate the dependence of the THR and PSD, for both the NMPC and PID controllers. Each point corresponds to the average values of these two variables for each run (one 24-hour period). It can be seen that for both controllers, there is negative correlation between these two variables. Under PID control, the values of the THR can be estimated by the values of the PSD and vice versa. Similar relation remains for the NMPC controller, but the relation is not as strong as for PID control. The absolute values of the correlation coefficients for both controllers are close to 1. The two variables are dependent for both controllers.

7.7.2.2.2 Joint performance function of the throughput rate and particle size As far as the JPF of the THR and PSD is considered, the preferable option is the value of the mineral recovered in the downstream flotation circuit. According to Eq.(6.1), when the product of the period of time, mineral price and head grade is constant and noted as $l$, the recovered mineral value depends on the THR and recovery. Further, due to the steady-state relation between the recovery and PSD, the recovered mineral value depends on the THR and PSD. In order to simplify the notation, in the rest of this subsection, $x$ and $y$ will be used alternatively to represent the THR and PSD respectively. Hence, the JPF of the $x$ and $y$ are

$$
\begin{aligned}
\vartheta(x, y) & =l \times x \times \text { recovery } \\
& =l \times x \times \vartheta(y)
\end{aligned}
$$




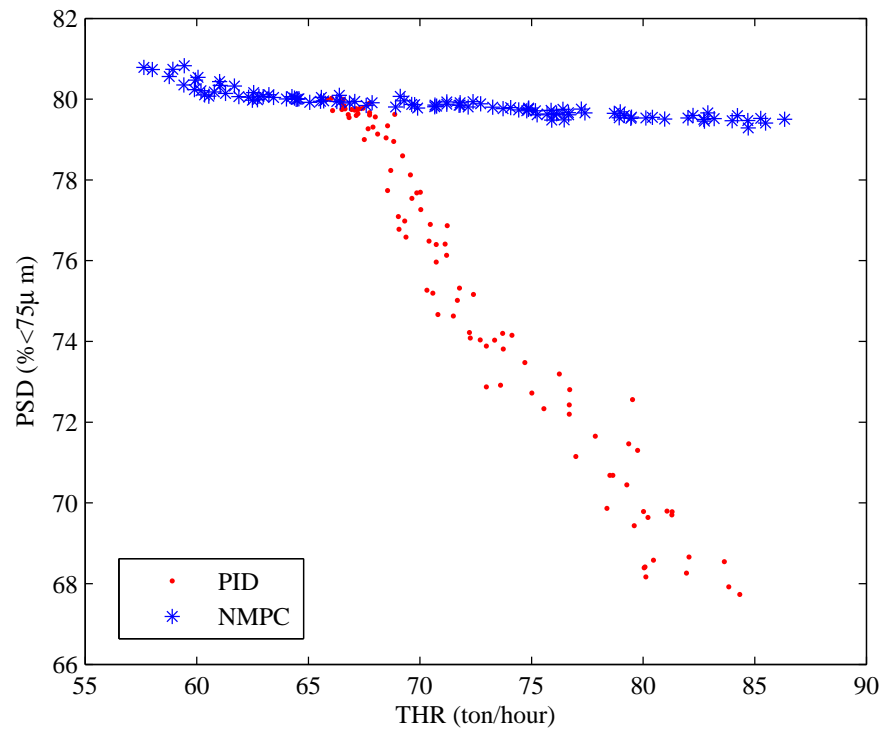

Figure 7.19: Scatter plot for the THR and PSD

where the $v(y)$ refers to subsection 6.4.2.

7.7.2.2.3 Joint probability density function of the throughput rate and particle size Normal distribution is assumed for the JPDF of the THR and PSD. The JPDF is

$$
f(x, y)=\frac{1}{2 \pi \sigma_{x} \sigma_{y} \sqrt{1-\rho^{2}}} \exp \left\{-\left[\left(\frac{x-\mu_{x}}{\sigma_{x}}\right)^{2}-2 \rho\left(\frac{x-\mu_{x}}{\sigma_{x}}\right)\left(\frac{y-\mu_{y}}{\sigma_{y}}\right)+\left(\frac{y-\mu_{y}}{\sigma_{y}}\right)^{2}\right] / 2\left(1-\rho^{2}\right)\right\}
$$

$\mu_{x}$ and $\mu_{y}$ mean the average value of the times series for the THR and PSD respectively. $\sigma_{x}$ and $\sigma_{y}$ mean standard deviation of the times series for the THR and PSD respectively. $\rho$ is the correlation coefficient of the time series of these two random variables.

7.7.2.2.4 Multivariate economic assessment for both controllers The multivariate economic assessment is conducted, based on the JPDF and JPF of the THR and PSD, using the following Eq.:

$$
\text { Mineral Value }=\int_{y_{\min }}^{y_{\max }} \int_{x_{\min }}^{x_{\max }} \vartheta(x, y) \times f(x, y) d x d y
$$

For each run of the NMPC and PID control, Eq.(7.91) is used to obtain the average recovered mineral value. Then the overall average recovered mineral value for NMPC controller is obtained by averaging the recovered values associated with 103 runs of the NMPC controller. Similarly for PID control with 97 runs. The overall average recovered value is $5063.87 l$ for NMPC and $5007.93 l$ for PID control. The standard deviations for the values are $287.35 l$ for NMPC and $224.43 l$ for PID control. It can be seen that more mineral is recovered with the NMPC controller, which bears considerable economic impact in practice. In order to reveal whether the improvement is statistically significant, a hypothesis test is conducted. In the test, $\omega$ is used to indicate the recovered value. The relevant null and alternative hypotheses are:

$H_{0}: \mu_{\omega_{P I D}}=\mu_{\omega_{N M P C}}$ 
$H_{1}: \mu_{\omega_{P I D}}<\mu_{\omega_{N M P C}}$

The NMPC controller will then be considered to help recover more mineral if $H_{0}$ can be rejected with a sufficient degree of significance. The significance level is also selected as $\alpha=0.15$ in this test. An $F$-test shows that the two samples of the recovered-mineral value are from the same population, since the value of $s_{P I D}^{2} / s_{N M P C}^{2}$ is 0.61 , which is less than $F_{i n v}(0.85,96,102)(1.23)$, i.e. a standard $t$-test can be conducted. The statistic $t$ is

$$
\begin{aligned}
t & =\frac{\mu_{\omega_{N M P C}}-\mu_{\omega_{P I D}}}{\sqrt{\sigma_{1}^{2} / n_{1}+\sigma_{2} / n_{2}}} \\
& =\frac{5063.87-5007.93}{\sqrt{287.35^{2} / 103+224.43^{2} / 97}} \\
& =1.54
\end{aligned}
$$

Since the value of $t$ is bigger than that of $T_{i n v}(0.85,198)$, which is 1.04 , the null hypothesis can be rejected with significance level 0.15. The recovered mineral value due to the NMPC controller is bigger than that for PID controllers with 0.85 confidence.

Furthermore, besides the recovered mineral value obtained above, other cost/profit factors can also influence economic performance, e.g. the reduction of energy consumption, the controller installation cost, the control software and hardware cost. Because of the limitation on simulation study, these elements are not considered further in this chapter, but are significant in practice.

\subsection{Discussion}

Generally, owing to improved control, the setpoint of CVs can be moved to a more profitable operating point, and the benefit results from reducing the $\mathrm{CV}$ variance and from moving the operating point in a more profitable direction. The PID controllers in this chapter cannot run the milling circuit at the same operating point as the NMPC controller does for the persistent disturbance scenario. This means that although the setpoint for both controllers is the same, they operate at different operating points, e.g. for the PSD in the statistical experiment, NMPC operates at 79.87 (passing $75 \mu \mathrm{m}$ ) and PID operates at 75.25 (passing $75 \mu \mathrm{m}$ ). The economic improvement of NMPC, compared to PID, therefore comprises two parts, namely a reduction in $\mathrm{CV}$ variance and operating at a more profitable operating point.

It is interesting to examine by how much the improvement in recovery results from the variance reduction and the improvement in operating point respectively. Taylor expansion can be used for this purpose (Chatfield, 1983). The average performance is related to the operating point, the variance of the time series of $\mathrm{CVs}$ and the characteristic of the relevant performance functions. The proof is given as is done for the performance function of concentrate grade in Craig and Koch (2003), from which it can be seen that

$$
\begin{aligned}
\bar{y} & =f(\bar{x})+0+\frac{1}{2} f^{\prime \prime}(\bar{x}) s^{2}+\text { Rem } \\
& \approx f(\bar{x})+\frac{1}{2} f^{\prime \prime}(\bar{x}) s^{2}
\end{aligned}
$$

For the PF of the PSD, as shown in Fig. 6.4, the first derivative is $2 a x+b$ and the second derivative is $2 a(-0.02)$ in the range of interest. Because the third and higher derivatives are all 
0, the Rem becomes 0 . Therefore

$$
\bar{y}=f(\bar{x})-0.01 s^{2}
$$

Note that the PID controllers run the circuit at $\bar{x}_{P I D}$ and NMPC at $\bar{x}_{N M P C}$, and accordingly the standard deviations $s_{P I D}$ and $s_{N M P C}$ respectively. Then

$$
\begin{aligned}
\bar{y}_{P I D} & =f\left(\bar{x}_{P I D}\right)-0.01 s_{P I D}^{2} \\
\bar{y}_{N M P C} & =f\left(\bar{x}_{N M P C}\right)-0.01 s_{N M P C}^{2}
\end{aligned}
$$

The recovery improvement is then

$$
\bar{y}_{N M P C}-\bar{y}_{P I D}=\left[f\left(\bar{x}_{N M P C}\right)-f\left(\bar{x}_{P I D}\right)\right]-0.01\left(s_{N M P C}^{2}-s_{P I D}^{2}\right)
$$

It can be seen that the difference in average recovery depends on the operating point and variable variance. Furthermore, owing to the coefficient -0.01 (resulting from the small second derivative of the $\mathrm{PF}$ ), the influence of the reduction of the $\mathrm{CV}$ variance on average recovery is small in this case. The improvement in recovery therefore mainly results from the improvement in the operating point. If the two operating points are the same, the average recovery will be very close. This result is of course heavily dependent on the characteristics of the PF of the PSD.

\subsection{Conclusion}

In this chapter, an economic performance assessment for three ROM ore milling circuit controllers has been cast in a recently proposed framework for the economic evaluation of advanced control systems. Performance functions of the milling circuits, which were developed a priori, play a key role in the assessment.

The assessment indicated that the economic performance of NMPC is better than that of the other two controllers with respect to recovery and recovered mineral value in the downstream flotation circuit for the persistent disturbance scenario. The reason is that, because disturbance rejection is significantly improved, the NMPC controller can operate at an operating point closer to the setpoint. In addition, the reduced variance of PSD under NMPC can also contribute to economic improvement. However, in this case the $\mathrm{CV}$ variance reduction only contributes a small part of the economic improvement owing to the shape of the PF used. 


\section{Chapter 8}

\section{Investigation of the performance functions for the final stage of a bleach process}

\subsection{Introduction}

The PFs for the final stage of a bleach plant are developed in Zhou (2002) and used for the EPA of the stage. The objective of the assessment is to determine whether the economic performance will be improved substantially with a control system improvement. The PFs of the process play an essential role in the assessment. The development of the PFs is reviewed critically below and the ideas used for the development are abstracted.

\subsection{Process description}

Bleaching is a process to increase the brightness of cellulosic materials. The required brightness can be reached by controlling retention time, temperature, $p H$, etc. Alberta-Pacific is one of the first kraft pulp mills particularly devised to incorporate $100 \% \mathrm{ClO}_{2}$ bleaching, in which chemicals are charged sequentially with intermediate washing between stages, as it is necessary to realise the desired removal or decolourisation of lignin in several stages. Four stages $D_{N}$, $D_{0}, E_{o p}$ and $D_{2}$ are designed to achieve the required objective.

$\mathrm{D}_{2}$ is the final stage of bleaching where $\mathrm{H}_{2} \mathrm{SO}_{4}$ and $\mathrm{ClO}_{2}$ are added to the pulp according to $p H$ and brightness of the feed from the $E_{o p}$ stage, as shown in Fig. 8.1. After around two hours of reaction, the high brightness pulp goes through the final washer and then reaches the pulp machine system. This stage is absolutely crucial in the whole bleaching process. The bleaching process is monitored by measuring pulp lignin content, pulp brightness and residual chemicals. On-line sensors and process control algorithms are used. The control of the $D_{2}$ stage is expected to achieve a high pulp brightness and several other secondary objectives, such as high brightness stability, pulp cleanliness and cellulosic content. 


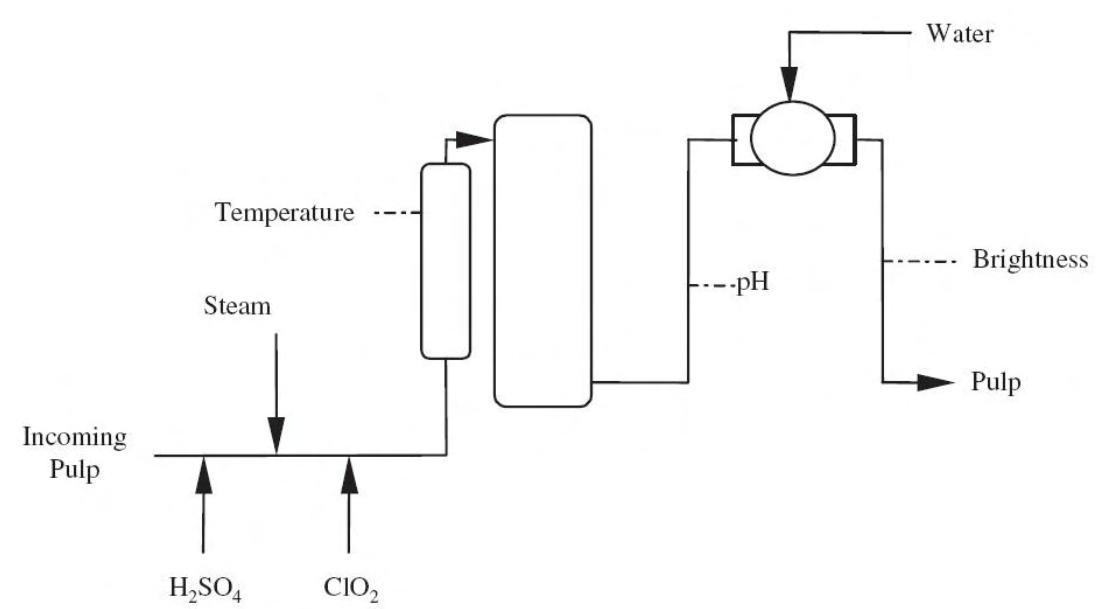

Figure 8.1: Schematic of the $D_{2}$ stage (Zhou, 2002)

\subsection{Performance function development for the $D_{2}$ stage}

The control systems for bleaching usually involve both feedback and feedforward control loops. The manipulated variables include $\mathrm{ClO}_{2}$ flow, $\mathrm{H}_{2} \mathrm{SO}_{4}$ flow, dilution water flow and steam flow. The CVs include brightness, lignin content, $p H$, viscosity, temperature, chemical residue and dirt. All these process variables relate directly or indirectly to the product quality and/or cost. In this analysis, mainly the brightness $(B)$, tower temperature $(T)$ and $p H$ after the retention tower are considered.

Bleaching costs are comprised of the costs of $\mathrm{ClO}_{2}, \mathrm{H}_{2} \mathrm{SO}_{4}$, steam, electric power, etc. Economic information, such as the market price for the product and operating cost, was obtained. For the purpose of the study, only the costs of chemicals and steam is considered. The economic performance involves two parts: sold product price and operating costs, which are investigated below.

The pulp is valued in terms of brightness and dirt percentage. There are three main products: high brightness pulp, normal brightness pulp (hardwood) and normal brightness pulp (softwood). The price of the normal brightness product (hardwood) is $\$ 500 / t$. When the brightness is a little lower ([88\%ISO $90.5 \% I S O])$, the pulp will be repulped with higher brightness pulp; its estimated price is $\$ 300 / t$. The pulp with very high brightness needs a large chemical charge, which considerably decreases its profitability. Moreover, pulp with too low a brightness needs too much high brightness pulp to repulp it, which makes the processing economically infeasible. Considering the cost of the $\mathrm{ClO}_{2}$ charge, royalty of producing chemicals and other costs (e.g. sale cost), the profitability of the pulp at specification is $\$ 50 / t$. The maximum profitability of off-specification pulp is $\$ 30 / t$. The profitability of very high or low brightness pulp is $-\$ 10 / t$. Overall, the PF for brightness, as shown in Fig. 8.2, is:

$$
v(B)= \begin{cases}-10, & B<85 \% I S O \\ 7.27(B)-628.18, & 85 \% I S O \leq B \leq 90.5 \% I S O \\ -17.14(B)+1601.43, & 90.5 \% I S O \leq B \leq 94 \% I S O \\ -10, & B>94 \% I S O\end{cases}
$$

As for the operating cost, since the $\mathrm{ClO}_{2}$ cost was included in the brightness PF, only the 


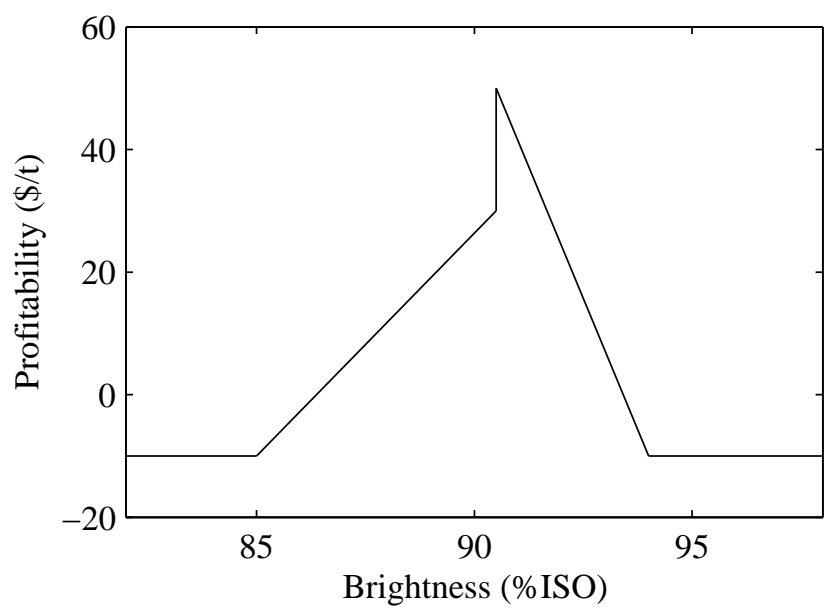

Figure 8.2: Performance function of brightness

costs of steam and $\mathrm{H}_{2} \mathrm{SO}_{4}$ are considered here. When the influence of $p H$ and temperature on brightness is not taken into account, the $\mathrm{PF}$ is assumed linear for simplicity. A low operating temperature means low steam cost and a high $\mathrm{pH}$ means a low $\mathrm{H}_{2} \mathrm{SO}_{4}$ charge. However, the temperature must be maintained within a range where the expected brightness can be obtained. For more accurate modelling, the relationship between brightness and temperature and that between brightness and $p H$ need to be determined. In this sector, the accuracy is limited because of the inaccuracy of the collected economic data. The PF of the temperature is:

$$
v(T)=0.1667(T)-4.8333
$$

The PF of the $p H$ is:

$$
v(p H)=-0.7565(p H)+10.2756
$$

Constraints need to be considered in an economic evaluation. In this study, only product specification and operating limits are examined, whereas e.g. product demand, throughput and availability of feed are assumed to be unconstrained. The constraint for brightness is:

$$
\operatorname{Pr}(B \geq 90.5 \% I S O) \geq 90 \%
$$

The constraints for temperature are:

$$
\operatorname{Pr}\left(68{ }^{\circ} \mathrm{C} \leq \mathrm{T} \leq 72^{\circ} \mathrm{C}\right) \geq 90 \%
$$

The constraints for $p H$ are:

$$
\operatorname{Pr}(4.2 \leq p H \leq 5.2) \geq 90 \%
$$

\subsection{Joint performance function}

The interaction of the economic impact of the brightness, $p H$ and temperature is not taken into account in Section 8.3, which assumes the profitability of brightness was not influenced by the temperature or $p H$. However, it is stated in the analysis that for more accurate modelling, the multivariate nature of the process needs to be considered. The nature is investigated in Zhou and Forbes (2003) where, for the same bleaching process, a JPF is developed. The JPF is 
derived from careful analysis of the product value versus production costs, as given in Eq. (8.7). The JPF is carefully scaled to sanitize the data.

$$
v(T, B, p H)= \begin{cases}-0.017(T)-0.076(p H)+1.5, & B \leq 85 \% I S O \\ -0.73(B)-0.017(T)-0.076(p H)+62, & 85 \% I S O \leq B \leq 90.5 \% I S O \\ 1.7(B)-0.017(T)-0.076(p H)-160, & 90.5 \% I S O \leq B \leq 94 \% I S O \\ -0.017(T)-0.076(p H)+1.5, & B \geq 94 \% I S O\end{cases}
$$

\subsection{Conclusion}

There are several ideas that can be generalised as follows:

- Profitability can be defined to describe economic performance of a process and for this purpose a profile of benefits and costs needs be established. The premise is that the product of this process can be sold directly.

- For each economically sensitive process variable, the possible benefit and cost need to be investigated comprehensively, and then integrated together into an overall index with a specific physical meaning.

- Some assumptions need to be made to facilitate PF development. Without assumptions, the relationships in the process could be too complex to quantify. Moreover, the available data restrict the accuracy of the modelling.

- Not all of the CVs are economically significant, because some process variables are controlled for other reasons, not necessarily economic ones. Thus, it is not necessary to take these variables into account in PF development.

- Costs that remain constant, however well the control system works, could be ignored when defining the relative economic performance of a process.

- When there is more than one grade for the product, benefits and costs need to match for the same grade of product. 


\section{Chapter 9}

\section{Methodology for developing performance functions}

\subsection{Introduction}

As clarified in previous chapters, PFs play a crucial role in the latest techniques of EPA of control systems. However, PFs are often difficult to build, varying from process to process and even changing for the same process with changes in extraneous economic conditions. In-depth understanding of the process operation and economics and sales agreements is required. In this chapter, a methodology for developing PFs is proposed based on the PF development of the EAF, grinding mill circuit and one stage of a bleach plant and a literature study of economic analysis. The methodology is reported in Wei and Craig (2009a). This methodology includes the following stages:

Stage 1: Determine information required for PF development.

- Process operation and control understanding (discussed in Section 2.1).

- Process economics understanding (discussed in Section 2.2).

Stage 2: Gain required information on PF development.

- PF-related information elicitation using survey research (Survey theory and techniques are discussed in Section 5.3).

- PF-related information available in the literature, including textbooks, journal papers, conference papers.

- PF-related information from plant tests.

Stage 3: Obtain suitable performance measures.

Stage 4: Make suitable assumptions.

Stage 5: Determine PFs.

Stage 6: Develop JPFs.

Without good understanding of the process being examined, it would be impossible to understand the process economics properly. Process economics information can be derived in mainly three ways, i.e. survey research, literature survey and experimental tests. Of these three ways, survey research is most popularly used. In this chapter, by default, the preferred way of gaining required information is survey research, either by questionnaire distribution and collection or by interview. In all process economics information, performance measures of the process that are supposed to be in accord with management strategy need to be ascertained before further 
modelling work. When the economic model is complex, it is necessary to make assumptions to facilitate the PF development without losing much accuracy. When the multivariate nature of the economic model is considered, JPFs need to be developed.

\subsection{Stage 1: Determine information required for perfor- mance function development}

The first stage of PF development is to determine the information required for the development. The information consists of three aspects, i.e. process operation, process control and process economics. Without in-depth understanding of process operation, it is impossible to gain sufficient understanding of process control. Without thorough insight into process operation and control, it is not easy to have in-depth understanding of process economics.

Preliminary operation and economic analysis refer to Section 2.1. For process operation, it is necessary to clarify production objectives, process description, feedstocks used for the process, energy used for the process, general disturbances and measurement instrumentation. For process control, it is necessary to know the selection of controlled and manipulated variables, the considerations for selecting these variables, the specifications for the controlled variables, the actuators serving the manipulated variables and the compensative action taken when the controlled variables violate their specifications. For process economics, it is necessary to know the economic impact when the controlled variables violate their specifications, including what the possible benefits will be and how the operational cost is changed. These general principles of extracting this information look simple, but it is very difficult to fulfil properly in practice.

How to derive this information is the task of stage 2 of the methodology. In the procedure proposed in this chapter, a systematic manner for this purpose is proposed, namely using survey research, literature survey and plant tests. One point of the methodology proposed here for PF development is to work out the PFs based on the generally recognised information about process operation, control and economics, whereas the elicitation of this information may not be conducted satisfactorily without systematic endeavour.

Besides the primary economic analysis, operating cost distribution needs to be focused on. Operating cost mainly involves energy and feedstocks. Energy may be chemical, nuclear, electrical, mechanical, thermal or radiant. When a controller drives manipulated variables to improve or maintain the performance of a process, the manipulated variables are often related to the energy/feedstock additions, which are related to monetary values. For instance, modifying the flow rate of some chemicals or electricity input will involve a transient change of the overall operating cost. Hence, for the process being investigated, the operating cost distribution needs to be established, as given in Table 9.1. The significance of establishing this cost distribution table is that, when manipulated variables are used for compensative action, the change in operating cost can be quantified easily, based on the 'nominal' operating cost; a good example can be seen in Oosthuizen et al. (2004).

In some processes (noted as $A$ in this context), a large proportion of economic consequences when CVs deviate from their setpoints lies in downstream processes (noted as $B$ in this context). Additional considerations are consequently necessary. A unifying study investigating the steady-state relationships between $\mathrm{CVs}$ of process $A$ and responding variables of process 
Table 9.1: Operational cost distribution for an electric arc furnace (Oosthuizen et al., 2004)

\begin{tabular}{|l|c|}
\hline Cost component & Percentage \\
\hline Scrap & $29.7 \%$ \\
\hline DRI & $22.3 \%$ \\
\hline Electric power & $14.9 \%$ \\
\hline Maintenance and other costs & $14.0 \%$ \\
\hline Electrodes & $7.5 \%$ \\
\hline Refractories & $3.7 \%$ \\
\hline Flux & $3.6 \%$ \\
\hline Labour & $1.9 \%$ \\
\hline Investment & $1.6 \%$ \\
\hline Oxygen & $0.7 \%$ \\
\hline Graphite & $0.1 \%$ \\
\hline
\end{tabular}

$B$ can be used. For each economically crucial CV (noted as $y_{i}$ in the following analysis), some information needs to be elicited, including how its fluctuation influences the cost and profit of processes $A$ and $B$; a table as given in Table 9.2 can be used.

Table 9.2: Economic consequences associated with $y_{i}$

\begin{tabular}{|l|l|l|l|}
\hline $\begin{array}{l}\text { Economic } \\
\text { impact }\end{array}$ & $\begin{array}{l}\text { Within specifi- } \\
\text { cation }\end{array}$ & $\begin{array}{l}\text { when bigger than upper } \\
\text { limit or setpoint }\end{array}$ & $\begin{array}{l}\text { when smaller than lower } \\
\text { limit or setpoint }\end{array}$ \\
\hline$\Delta P_{A}\left(y_{i}\right)$ & & & \\
\hline$\Delta P_{B}\left(y_{i}\right)$ & & & \\
\hline$\Delta C_{A}\left(y_{i}\right)$ & & & \\
\hline$\Delta C_{B}\left(y_{i}\right)$ & & & \\
\hline
\end{tabular}

\subsection{Stage 2: Gain required information on performance function development}

After determining what information is required in stage 1, the task of stage 2 is to obtain this information. There are three main ways that can be used for this purpose, i.e. survey research, literature survey and plant test, which are discussed respectively below.

\subsubsection{Performance function-related information elicitation using sur- vey research}

Survey research is the most popular way of deriving expert knowledge that is required to develop PFs (Sapsford, 2007). As shown in Bauer and Craig (2008), Oosthuizen et al. (2004) and Wei and Craig (2009c), survey research plays a key role in obtaining instructive information. In order to gain in-depth understanding of the process operation and economics that are crucial for the PF development, a survey study can be used. Survey theory and relevant techniques are discussed in Section 5.3. 


\subsubsection{Performance function-related information available in the lit- erature}

Publications describing the first principle model of the process being considered may carry useful information. The economic value of the energy and materials, which may be included in the process model, may contribute to the establishment of a PF. PF-related information is often available during the plant design and test phases and appears in the literature. Examples can be seen in Wills and Napier-Munn (2006) and Friedmann (2006). This information is highly meaningful in PF development. For example, for the PF of concentrate grade in flotation circuits, as described in Wills and Napier-Munn (2006), it remains the same for various flotation circuits despite the variety of circuit configurations and control structures. Although the parameters of the functions representing the relationship have different values in different processes, the relationship can still be used for reference.

\subsubsection{Performance function-related information from plant tests}

Plant tests can be viewed as a method for deriving PFs, in which the process considered is managed to reach steady states under various combinations of variables for which a steady state relationship is intended to be built, while keeping other variables and parameters as constant as possible; an example of constructing a residue vs grind relation is given in Craig et al. (1992b). The economic impact of one variable on another one may then be discovered from the collected steady-state operating data. However, this method is often infeasible in practice, as this kind of plant test is often extremely time-consuming and costly. Another reason is that sometimes the influence of disturbances on the system will overwhelm the variation of process variables. Furthermore, in order to conduct the tests, normal running of the system needs to be stopped for quite a while, which may lead to considerable loss. These plant tests can, however, be conducted in a simulation study that provides a disturbance-free environment so that the relationships between the process variables and the economically sensitive variables can be discovered relatively easily (Sosa-Blanco et al., 2000). This simulation study, based on precise process models (Friedmann, 2006), is advisable to consider disturbances sufficiently in order to make the simulation more realistic.

\subsection{Stage 3: Select suitable performance measures}

When the process focused on is a sector of a huge plant, the economic performance measures, which are intended to be used to assess the performance of a control system, need to be selected carefully, as there may be several possible options available, such as production rate, product yield, product quality, profitability, etc, but which one is suitable is not clear. Sometimes, a control system is driven to optimise the operation cost, whereas from the management viewpoint the most important thing is to increase the throughput as much as possible.

The effectiveness of any performance measurement system must be treated as a critical factor in the success of the execution of its strategy. It is important that the performance measures represent the corporate strategy and the resulting strategy of the manufacturing operation. The corporate strategy needs to be realised from the uppermost level to the bottom level. "Voll- 
mann decomposition' provides a good method of analysing and developing effective corporate performance measurement systems (Martin, 2005). 'Vollmann decomposition' is the iteration of 'Vollmann triangles'. At the top of this triangle is the plant's manufacturing strategy, which should be clearly stated and communicated to all the people in the operation who are supposed to support this strategy. Out of the strategy should come an action plan that should indicate how the strategy should be realised. The performance measures should be established to reflect and assess whether the action plan is successful. Performance measures should provide the link between the activities of the business and the strategic planning process. If the performance measures reflect the strategy and are built at all levels of the organisation, the plant personnel will tend to work to reach the measures and accordingly the plant's strategy will be executed efficiently.

The Vollmann triangle can be taken as a continuous improvement model and represents a cycle starting with the corporate strategy. Once market conditions require a new strategy, the Vollmann triangle cycle can be conducted over again and cause continual improvement in economic performance in line with the competitive strategy. The Vollmann decomposition is achieved by top-down repetition of the Vollmann triangle model.

\subsection{Stage 4: Make suitable assumptions}

Assumptions are very important for PF development. The PF model of the process may be very complex so that it is very difficult to quantify. There may be more than one economic consequence when a $\mathrm{CV}$ deviates from the setpoint or desired range, and the relationship between the variable and some consequences may be very obscure, or be noted seldom by the relevant engineer or superintendent or accounting division, or be unimportant because the consequence is minor compared to the dominant one. Assumptions then need to be made in order to facilitate the modelling, which may otherwise be difficult to handle. They can help to simplify the PF modelling problem to a reasonable extent where it becomes operable without losing much accuracy. What assumptions need to be made deserves serious consideration. However, there are no general rules and the assumptions should be made according to the specific situation of the process. For example, the assumptions may be:

- The wisdom of neglecting some minor economic impact is sometimes required. Neglecting economic consequences that are small compared to the dominant consequence and are difficult to quantify, will simplify the development of PFs without losing much accuracy.

- The economic consequences of different CVs are independent.

- The PF of a CV is linear (or quadratic or some other type) in a range.

- The process investigated is a bottleneck in the whole plant.

- There are no constraints for some variables.

- Only some of all CVs are economically important.

- Only some operating costs are significant.

- When the production quality is improved, the throughput rate remains unchanged. 


\subsection{Stage 5: Determine performance functions}

Once the preceding steps have been executed properly and the required information has been obtained, the PFs can be determined by synthesising all the collected information. Some suggestions are given below:

- The costs that remain constant, however well the control system works, could be ignored when defining the economic performance of a process, as the constant costs cannot help to reflect the distinction in economic performance of different controllers.

- When there is more than one grade for the product, benefits and costs need to match for the same grade product.

- Profitability can be defined to describe the economic performance of a process, based on which benefits and cost scenarios can be listed. The premise is that the product of this process can be sold directly.

- For processes in which CVs are required to stay within specifications at batch time and in which there is no additional benefit, however well the controller works, the additional cost incurred to compensate for the deviation of CVs from specifications may be a suitable option of PFs. It needs to be individualised for each CV according to the specifics of the process being explored.

- Even for the same process, PFs may change when the process's extraneous economic environment changes, e.g. the market's quality demand, the market's throughput demand, a change in the feedstock's price, a change in the price of electricity, etc. Hence, PFs need to be updated regularly to adapt to these conditions.

- It is difficult to find generic PFs of the same type in different processes in that performance measures or process configuration may be different, etc.

- In different plants, even for the same kind of processes, the focus of economic performance may be different, depending on the upper level strategies.

- For each economically sensitive process variable, the possible benefit and cost need to be investigated comprehensively, and then integrated into an overall index with a specific physical meaning.

- Not all of the CVs are economically significant, because some of the process variables are controlled for other reasons, not necessarily economic ones. Thus, it is not necessary to take these variables into account in PF development.

- Generally for a PF there are two possible options, i.e. using real plant data or using sanitised plant data expressed as percentage values. Real plant data are often difficult to obtain as these are usually confidential. This argument is confirmed in Zhou and Forbes (2003) where only brief results are given and detailed information is protected. Hence, taking the first option tends not to be practical for academic research, but it is ideal for an internal evaluation of an industrial plant. Alternatively, the second option can be used, where the percentage values of $\Delta C / \Delta P$ are divided by a nominal value such as the average operating cost of the process for a given period. This is the case in Oosthuizen et al. (2004), where the PFs are expressed as percentage values of additional costs divided by the typical operating cost for a tap of an electric arc furnace. The use of percentage values has the advantage that sensitive production data can be protected and are consequently easier to obtain. 


\subsection{Stage 6: Develop joint performance functions}

When the economic impact of different CVs is considered separately, IPFs need to be developed. The underlying assumption is that the CVs are independent, which is often not true. If two CVs violate their specifications simultaneously, their economic consequences could overlap or even be the same, not necessarily the sum of two independent ones. Not considering the dependence of CVs may consequently result in biased estimation of economic performance of a controlled system.

However, when the economic model's multivariate nature is considered, i.e. the interaction of economic impact of different CVs is considered, JPFs need to be developed. JPFs may help to determine a more profitable optimal operating point. Considering the multivariate nature of the process, the economic model may result in a more accurate estimate of economic performance of a controlled system. A decision needs to be taken at the beginning of an EPA whether to develop JPFs or IPFs, which has a significant influence on some decisions that need to be made later. JPFs can be established from scratch by following the procedure mentioned above or be built based on the IPFs that have already been established.

For both cases, the dependence of the CVs needs to be investigated first. The scatter plot is a powerful tool for examining the relationship between two variables (Moore and McCabe, 1989). For the time series of every two $\mathrm{CVs}$, the data points are plotted in a two-dimensional plane in which the $\mathrm{x}$-axis is for one variable and the $\mathrm{y}$-axis for the other variable. The scatter plot can be explained by inspecting an overall pattern using visual examination. The pattern can manifest the direction, shape and strength of the dependence of two variables. Two variables are regarded as being associated positively when above-average values of one variable are likely to coexist with above-average values of the other variable, and below-average values tend to take place together. Two variables are taken as being associated negatively if above-average values of one variable coexist with below-average values of the other variable, and vice versa.

Based on the dependence of CVs JPFs for dependent CVs are developed. Also, the survey study and literature survey are two recommended ways of obtaining the required information. If it is decided to develop JPFs by updating IPFs, the focus should be put on the interaction of economic consequences that can be seen from IPFs. An issue that would then deserve attention is that if the costs associated with compensative action of two CVs have overlapping parts, meaning the compensative action will help to recover both CV simultaneously, these parts should not appear twice in the JPF of these two CVs.

\subsection{Conclusion}

PFs have an central role to play in the latest techniques of EPA. A methodology of PF development is proposed in this chapter. It starts from the in-depth understanding of process operation and proceeds with insight into process control and then process economics. The required information may be obtained mainly in three ways, namely survey study, literature survey and plant tests. Then, after determining suitable performance measures and making appropriate assumptions, PFs can be established based on the required information and some instructive suggestions. Moreover, the development of JPFs is discussed. 


\section{Chapter 10}

\section{Conclusions}

\subsection{Summary}

The contributions of this thesis are summarised as follows. Firstly, a methodology of developing PFs was proposed based on the development of PFs for an electric arc furnace (a batch process), a grinding mill circuit (a continuous process) and a stage of a bleach plant (a continuous process). The second is that a detailed EPA of three controllers (an NMPC controller, a decentralised controller and three single-loop PID controllers) of a grinding mill circuit was conducted, using a recently published framework to show the central role of PFs in EPA of control systems and how to perform an EPA systematically. It was also shown that the NMPC controller provided better economic performance in terms of recovery in the downstream flotation circuit and the value of the mineral recovered in the flotation circuit for persistent disturbances. Besides, it was illustrated that the improvement in the economic performance under the NMPC mainly resulted from the improvement of the operating point, and the controlled variables' variation reduction only accounted for a small part of the overall improvement. Furthermore, web-based survey research was conducted and the current practice and guidelines on the control and functional/economic performance assessment of grinding mill circuits in mineral processing industries were captured.

An EPA of an MPC controller for an electric arc furnace was investigated in detail. The original assessment was based on the IPFs of the furnace's CVs and the IPFs were built mainly based on a plant interview. The development of these IPFs was reviewed critically and crucial ideas used were abstracted. Moreover, the dependence of the CVs was examined and then JPFs were established, based on the dependence profile and IPFs. A multivariate economic assessment was then conducted and showed that JPFs could help to provide a more accurate estimate of the economic performance of a controlled process.

A web-based survey study on grinding mill circuits in mineral processing industries was performed. One of its objectives was to derive general PFs of grinding circuits. The survey results carried instructive information for developing the PFs for grinding circuits. Furthermore, an in-depth literature survey was conducted and the relationship between the product's particle size distribution of grinding mill circuits and mineral recovery in downstream flotation circuits was found to be consistent in shape. The PFs of a grinding mill circuit being considered were developed by incorporating the information derived from the survey results and literature study. 
An investigation into the PF development of a stage of a bleach plant was performed and the primary ideas used for the development were elicited.

A methodology for PF development was then given by synthesising the methods used in developing PFs of the processes as described above. This methodology mainly includes the following stages:

Stage 1: Determine information required for PF development.

- Process operation and control understanding.

- Process economics understanding.

Stage 2: Gain required information on PF development.

- PF-related information elicitation using survey research.

- PF-related information available in the literature, including textbooks, journal papers, conference papers.

- PF-related information from plant tests.

Stage 3: Obtain suitable performance measures.

Stage 4: Make suitable assumptions.

Stage 5: Determine PFs.

Stage 6: Develop JPFs.

The economic performance of three controllers, i.e. the NMPC controller, decentralised controller and three single-loop PID controllers, of the grinding mill circuit being considered was assessed using a novel EPA framework. The assessment included two primary stages, i.e. before and after the implementation of the NMPC controller. The first stage started with the identification of the economic performance associated with the PID controllers, proceeded with the design of the NMPC controller and the estimate of the NMPC controller's economic performance, followed by an initial economic project evaluation. In the second stage, after the implementation of the NMPC controller, the assessment focused on how to determine the improvement in the economic performance resulting from the NMPC controller, using a carefully designed experiment when both the PID and NMPC controllers were in place. The grinding circuit's PFs played a key role in the assessment. The assessment showed how to perform an EPA of a control system systematically. It turned out that the NMPC controller could help to provide better recovery than the other two controllers for persistent disturbances. In addition, the NMPC was advantageous with regard to the value of the mineral recovered in the downstream flotation circuit. The EPA also showed that the economic performance's improvement under the NMPC had taken place primarily because of the improvement in the operating point. The controlled variables' variation reduction only made a small contribution to the overall improvement in view of the feature of the PF of the circuit's product PSD.

A web-based survey study was conducted and the current practice and guidelines on the control and functional/economic performance assessment of the grinding mill circuits were captured. The three variables that are controlled most frequently are the product particle size, the slurry level in the sump and the sump discharge slurry density. The manipulated variables used most commonly are the flow rate of water to the sump, the flow rate of water to the mill, the feed rate of solids to the mill and the flow rate of the sump discharge slurry. Fewer than half of the respondents quantified the economic impact of the controlled variables violating their setpoints in terms of mathematical functions. Different mathematical functions were sometimes selected for the same control variable. PID controllers are used predominantly in grinding mill circuits, and more than half of the respondents are satisfied with the performance of their control loops. Process control system benefits result mainly from process stability improvements, an increase in throughput and a reduction in energy consumption. The method used most popularly for 
estimating benefits is past experience with similar projects. The main sources of cost for a control system are control hardware, consultant manpower cost and technology cost. The costs are estimated mainly from experience with similar projects and installation cost as quoted by a vendor. Cost/benefit analysis is regarded to be important at all stages of the implementation of a control system, with the analysis before a controller is implemented considered more important than after the controller implementation and the follow-up analysis. One-third of the respondents stated that the economic assessment of grinding control needed to be improved.

\subsection{Assessment}

The methodology of developing PFs proposed in this thesis solves a fundamental problem in EPA of process control systems, i.e., establishing an economic model. Intuitively for an EPA of a control system, based on this methodology, the economic model can be built in an easy manner, which will make possible further systematic economic assessment, such as economic performance calculation, estimation, monitoring and optimisation.

The economic assessment of the milling circuit illustrates how to perform EPA systematically and the key role of PFs in the assessment. It also demonstrates how to investigate the source of the improvement in economic performance.

The work conducted in this thesis is a multidisciplinary study, which covers various knowledge areas. However, it is preferable that an in-depth analytical analysis be further conducted. The multivariate nature of processes has not been investigated in depth. Although the PF development methodology proposed accounts for the economic models of the three processes discussed in this thesis sufficiently, it may not fit into other processes well as the methodology may miss some characteristics of economics modelling. Its validity still needs to be examined and it to be enriched.

The PDFs are assumed to follow Gaussian distributions with justification in this thesis. However, this may not always be the case. Then, the PDFs need to be formed otherwise. In addition, the estimation of an improved control system's performance that is based on the assumption of Gaussian distributions of process variables also needs to be performed in a way different from what is used in this thesis.

\subsection{Future work}

There are still some aspects that need to be investigated but fall outside the scope of this thesis, including:

1. Software for developing PFs using artificial intelligence-based algorithms and objectoriented techniques is in demand. Because obtaining the information required for $\mathrm{PF}$ development is fairly complex, the availability of such software will greatly facilitate this development. Then, expert knowledge can be elicited in an easier way compared to an iterative interview. 
2. The effectiveness of the proposed methodology of developing PFs still needs to be examined and the methodology needs to be enriched. It can be applied to various processes to obtain PFs and then EPA can be conducted using the recommended framework. In addition, for the process being considered, both IPFs and JPFs can be developed and accordingly both univariate and multivariate EPA can be performed. It can subsequently be seen whether a multivariate assessment method improves the accuracy of the estimate of the controlled system's economic performance.

3. Control systems run to meet stipulated specifications that are determined by both economic and operational considerations. It is worth considering changing the objectives of control systems from satisfying functional specifications, such as setpoint tracking and disturbance rejection, to optimising the overall economic performance. 


\section{Reference}

Akande, S., Huang, B. and Lee, K. H. (2009). MPC constraint analysis - Bayesian approach via a continuous-valued profit function, Industrial and Engineering Chemistry Research 48: 39443954 .

Allen, D. H. (1991). Economic Evaluation of Projects, 3rd edn, Rugby: Institution of Chemical Engineers.

Alreck, P. L. and Settle, R. B. (1995). The Survey Research Handbook: Guidelines and Strategies for Conducting a Survey, 2nd edn, Burr Ridge: Irwin.

Anderson, L., Perry, R. and Neale, A. J. (1996). Application of deadtime and gain compensation to SAG feeder control at P. T. Freeport Indonesia, Proceedings of the 28th Annual Meeting of the Canadian Mineral Processors, Paper no. 24, Ottawa, Ontario.

Åström, K. J. and Hägglund, T. (1995). PID Controllers: Theory, Design and Tuning, Instrument Society of America.

Babbie, E. R. (1990). Survey Research Methods, 2nd edn, Belmont, Calif: Wadsworth.

Bascur, O. A. (1991). Integrated grinding/flotation controls and management, Proceedings of the Copper 91-Cobre 91, Vol. 2, pp. 411-427.

Bauer, M. and Craig, I. K. (2006). Economic performance assessment of APC projects - a review and framework, Proceedings of the SICOP Workshop, Gramado, Brazil.

Bauer, M. and Craig, I. K. (2008). Economic assessment of advanced process control - a survey and framework, Journal of Process Control 18(1): 2-18.

Bauer, M., Craig, I. K., Tolsma, E. and de Beer, H. (2007). A profit index for assessing the benefits of process control, Industrial and Engineering Chemistry Research 46: 5614-5623.

Bawden, N. M. (1993). The prediction, quantification and evaluation of advanced control benefits, Master's thesis, the University of Witwatersrand.

Bazin, C., Grant, R. and Duchesne, S. (1995). Simulation of flotation bank performances as a function of fineness of grind, Proceedings of 19th International Mineral Processing Congress, Vol. 3, p. 221.

Bazin, C., Grant, R., Cooper, M. and Tessier, R. (1994a). A method to predict metallurgical performances as a function of fineness of grind, Minerals Engineering 7(10): 1243-1251.

Bazin, C., Grant, R., Cooper, M. and Tessier, R. (1994b). The prediction of metallurgical performances as a function of fineness of grind, Proceedings of the 26th Annual Meeting of the Canadian Mineral Processors, Canadian Mineral Processors, Ottawa. 
Bekker, J. G. (1999). Modeling and control of an electric arc furnace off-gas process, Master's thesis, University of Pretoria.

Bekker, J. G., Craig, I. K. and Pistorius, P. C. (1999). Modelling and simulation of an electric arc furnace process, ISIJ International 39: 23-32.

Bekker, J. G., Craig, I. K. and Pistorius, P. C. (2000). Model predictive control of an electric arc furnace off-gas process, Control Engineering Practice 8: 445-455.

Borell, M., Bäckström, P. and Söderberg, L. (1996). Supervisory control of autogenous grinding circuits, International Journal of Mineral Processing 44-45: 337-348.

Bourassa, M., Lanthier, R., Beaudoin, P. and Chéfaï, S. (1995). Grinding circuit control optimization at mine Doyon, Proceedings of the 27th Annual Meeting of the Canadian Mineral Processors, Canadian Mineral Processors, Ottawa, pp. 295-310.

Bozenhardt, H. and Dybeck, M. (1986). Estimating savings from upgrading process control, Chemical Engineering 93(3): 99-102.

Brace, I. (2004). Questionnaire Design, London; Sterling, VA: Kogan Page.

Brisk, M. L. (2004). Process control: Potential benefits and wasted opportunities, Proceedings of the 5th Asian Control Conference, pp. 10-16.

Brisk, M. L. and Blackhall, N. (1995). Benchmarking for best practice, Process and Control Engineering pp. 44-49.

Chatfield, C. C. (1983). Statistics for Technology, 3rd edn, London: Chapman and Hall.

Chen, J. (2000). Logarithmic integrals, interpolation bounds, and performance limitations in MIMO feedback systems, IEEE Transactions on Automatic Control 45: 1098-1115.

Chen, X., Zhai, J., Li, S. and Li, Q. (2007). Application of model predictive control in ball mill grinding circuit, Minerals Engineering 20(11): 1099-1108.

Choudhury, M. A. A. S., Shah, S. L. and Thornhill, N. F. (2004). Diagnosis of poor control-loop performance using higher-order statistics, Automatica 40: 1719-1728.

Coetzee, L. C. (2009). Robust nonlinear model predictive control of a closed run-of-mine ore milling circuit, PhD thesis, University of Pretoria.

Coetzee, L. C. and Craig, I. K. (2007). Robust MPC of a run-of-mine ore milling circuit, Proceedings of the 7th IFAC Symposium on Nonlinear Control Systems, Pretoria, South Africa, pp. 904-909.

Coetzee, L. C., Craig, I. K. and Kerrigan, E. C. (2008). Nonlinear model predictive control of a run-of-mine ore milling circuit, Proceedings of the 17th IFAC World Congress. ISBN: 9783-902661-00-5. doi:10.3182/20080706-5-KR-1001.2587, Vol. 17, Elsevier, Korea, pp. 1062010625.

Coetzee, L. C., Craig, I. K. and Kerrigan, E. C. (2009). Robust nonlinear model predictive control of a run-of-mine ore milling circuit, IEEE Transactions on Control Systems Technology. doi:10.1109/TCST.2009.2014641.

Craig, I. K. and Henning, R. (2000). Evaluation of advanced industrial control projects: A framework for determing economic benefits, Control Engineering Practice 8: 769-780. 
Craig, I. K. and Koch, I. (2003). Experimental design for the economic performance evaluation of industrial controllers, Control Engineering Practice 11: 57-66.

Craig, I. K. and Macleod, I. M. (1995). Specification framework for robust control of a run-ofmine ore milling circuit, Control Engineering Practice 3(5): 621-630.

Craig, I. K. and Macleod, I. M. (1996). Robust controller design and implementation for a run-of-mine ore milling circuit, Control Engineering Practice 4(1): 1-12.

Craig, I. K., Hulbert, D. G., Metzner, G. and Moult, S. P. (1992a). Extended particle-size control of an industrial run-of-mine milling circuit, Powder Technology 73: 203-210.

Craig, I. K., Hulbert, D. G., Metzner, G. and Moult, S. P. (1992b). Optimized multivariable control of an industrial run-of-mine circuit, Journal of the South African Institute of Mining and Metallurgy 92: 169-176.

Czaja, R. and Blair, J. (2005). Designing Surveys: A Guide to Decisions and Procedures, 2nd edn, Pine Forge.

Desbiens, A., Flament, F. and Pomerleau, A. (1997a). Distributed control at the Kidd Creek grinding plant. Part II: Implementation, CIM Bulletin 90: 145-150.

Desbiens, A., Najim, K., Pomerleau, A. and Hodouin, D. (1997b). Adaptive control - practical aspects and application to a grinding circuit, Optimal Control Applications and Methods 18: $29-47$.

Deshpande, B. (1989). Multivariable Process Control, Instrument Society of America.

Diehl, M., Bock, H. G. and Schloder, J. P. (2005). A real-time iteration scheme for nonlinear optimization in optimal feedback control, SIAM Journal on Control and Optimization 45(3): 1714-1736.

Dubé, Y. and Hodouin, D. (1989). Adaptive control of a laboratory grinding circuit, Minerals Engineering 2(2): 245-258.

Edwards, R. and Vien, A. (1999). Application of a model-based size recovery methodology, in D. Hodouin, C. Bazin and A. Desbiens (eds), Control and Optimisation in Minerals, Metals and Materials Processing, The Metallurgical Society of CIM, pp. 147-160.

Edwards, R., Vien, A. and Perry, R. (2002). Strategies for the instrumentation and control of grinding circuits, in A. L. Mular, D. N. Halbe and D. J. Barratt (eds), Mineral Processing Plant Design, Practice, and Control, Society for Mining Metallurgy and Exploration, pp. 2130-2151.

Evans, G. (2001). A new method for determining charge mass in AG/SAG mills, Proceedings of the International SAG Conference, Vol. 2, Vancouver, pp. 331-345.

Evans, M. J. and Rosenthal, J. S. (2004). Probability and Statistics: The Science of Uncertainty, New York: Freeman.

Fallside, F. (ed.) (1977). Control System Design by Polo-Zero Assignment, Academic Press.

Feng, D. and Aldrich, C. (1999). Effect of particle size on flotation performance of complex sulphide ores, Minerals Engineering 12(7): 721-731. 
Fisher, R. A. and Yates, F. (1953). Statistical Tables for Biological, Agricultural and Medical Research, 4th edn, Edinburgh: Oliver and Boyd.

Flament, F., Desbiens, A. and del Villar, R. (1997). Distributed control at the Kidd Creek grinding plant. Part I: Control strategy design, CIM Bulletin 90: 139-144.

Flament, F., Thibault, J. and Hodouin, D. (1993). Neural network based control of mineral grinding plants, Minerals Engineering 6(3): 235-249.

Fowler, F. J. (2009). Survey Research Methods, 4th edn, Thousand Oaks: SAGE.

Freeman, N., Keenan, W., Hancock, S. and LePage, G. (1994). Grinding circuit control techniques and experiences, Proceedings of the 5th Mill Operators' Conference, pp. 193-200.

Friedmann, P. G. (2006). Automation and Control Systems Economics, 2nd edn, The Instrumentation, Systems, and Automation Society.

Galán, O., Barton, G. W. and Romagnoli, J. A. (2002). Robust control of a SAG mill, Powder Technology 124: 264-271.

Gaudin, A. M., Groh, J. O. and Henderson, H. B. (1931). Effect of particle size on flotation, American Institute of Mining and Metallurgical Engineering no.414: 3-23.

Goodwin, G. C., Graebe, S. F. and Salgado, M. E. (2001). Control System Design, Prentice Hall.

Grant, R., Bazin, C. and Cooper, M. (1991). Optimizing grind using size by size recovery analysis at Brunswick Mining, in D. Malhotra, R. R. Klimpel and A. L. Mular (eds), Evaluation and Optimization of Metallurgical Performance, SME/AIME, p. 329.

Hague, P. (1993). Questionnaire Design, London: Kogan PAGE.

Harris, T. (1989). Assessment of closed loop performance, Canadian Journal of Chemical Engineering 67: 856-861.

Harris, T., Boudreau, F. and MacGregor, J. F. (1996). Performance assessment using of multivariable feedback controllers, Automatica 32: 1505-1518.

Herbst, J. A., Rajamani, K., Miller, J. D. and Lin, C. L. (1988). Development of a multicomponent multisize liberation model, Minerals Engineering 1(2): 97-111.

Hodouin, D., Jämsä-Jounela, S.-L., Carvalho, M. T. and Bergh, L. (2001). State of the art and challenges in mineral processing control, Control Engineering Practice 9: 995-1005.

Huang, B. and Shah, S. L. (1999). Performance Assessment of Control Loops: Theory and Application, Springer, New York.

Huang, B., Ding, S. X. and Thornhill, N. (2005). Practical solutions to multivariate feedback control performance assessment problem: Reduced a priori knowledge of interactor matrices, Journal of Process Control 15: 573-583.

Huang, B., Lu, S. and Xu, F. (2006). Toward optimal MPC performance: industrial tools for multivariate control monitoring and diagnosis, Proceedings of the SICOP Workshop, Gramado, Brazil. 
Hulbert, D. G. (1989). The state of the art in the control of milling circuits, Proceedings of the IFAC Symposium on Automation in Mining, Mineral and Metal Processing, Argentina, pp. $1-10$.

Hulbert, D. G. (2002). Evaluation of the economic benefits of milling circuit control, in L. Basenez, J. A. D. la Puenta and E. F. Camacho (eds), Proceedings of the 15th IFAC Triennial World Congress, Elsevier Science, Barcelona, Spain.

Hulbert, D. G., Craig, I. K., Coetzee, M. L. and Tudor, D. (1990). Multivariable control of a run-of-mine milling circuit, Journal of the South African Institute of Mining and Metallurgy 90(7): 173-181.

Hull, D. G. (1997). Conversion of optimal control problems into parameter optimization problems, Journal of Guidance, Control, and Dynamics 20(11): 57-60.

Ivezić, D. D. and Petrović, T. B. (2003). New approach to milling circuit control - robust inverse nyquist array design, International Journal of Mineral Processing 70: 171-182.

Jelali, M. (2006). An overview of control performance assessment technology and industrial applications, Control Engineering Practice 14(5): 441-466.

Jiang, R. and Fruehan, R. J. (1991). Slag foaming in bath smelting, Metallurgical Transactions $B$ 22B(4): 481-489.

Jones, J. A., Deister II, R. D., Hill, C. W., Barker, D. R. and Crummis, P. B. (1991). Process control at the DOE Run Company, Proceedings of SME Plant Operator Symposium, Denver, Colorado.

Kawajir, Y., Laird, C. and Wachter, A. (2006). Introduction to Ipopt: A tutorial for downloading, installing, and using ipopt, Technical report, Carnegie Mellon University.

King, R. P. (1979). A model for the quantitative estimation of mineral liberation by grinding, International Journal of Mineral Processing 6: 207-220.

Lankford, W. T., Samways, N. L., Craven, R. F. and McGannon, H. E. (eds) (1985). The Making, Shaping and Treating of Steel, 10th edn, United States Steel.

Lant, P. and Steffens, M. (1998). Benchmarking for process control: Should I invest in improved process control?, Water Science and Technology 37(12): 49-54.

Latour, P. (2006). Demise and keys to the rise of process control, Hydrocarbon processing 85: $71-80$.

Latour, P. L. (1992). Quantify quality control's intangible benefits, Hydrocarbon processing 71: $61-68$.

Latour, P. L. (1996). Process control: CLIFFTENT shows it's more profitable than expected, Hydrocarbon processing 75: 75-80.

Latour, P. L., Sharpe, J. H. and Delaney, M. C. (1986). Estimating benefits from advanced control, ISA Transactions 65: 13-21.

Latour, P. R. (1976). The hidden benefits from better process control, Advances in Instrumentation 55: 1-11. 
Lo, Y. C., Oblad, A. E. and Herbst, J. A. (1996). Cost reduction in grinding plants through process optimization and control, Minerals and Metallurgical Processing 8: 19-21.

Lougee-Heimer, R. (2003). The common optimization interface for operations research, IBM Journal of Research and Development 47(1): 57-66.

Louw, J. J., Hulbert, D. G., Smith, V. C., Singh, A. and Smith, G. C. (2003). Mintek's process control tools for milling and flotation control, in L. Lorenzen and D. J. Bradshaw (eds), Proceedings of the XXII International Mineral Processing Congress, Cape Town, South Africa, pp. 1581-1589.

Lynch, A. J. (1979). Mineral Crushing and Grinding Circuits: Their Simulation, Design, and Control, Elsevier Science Ltd.

Lynch, A. J. (1981). Mineral and Coal Flotation Circuits: Their Simulation and Control, New York : Elsevier Scientific Pub. Co.

Lynch, A. J. and Bush, P. D. (1977). Mineral Crushing and Grinding Circuits: Their Simulation, Optimization, Design and Control, New York : Elsevier Scientific Pub. Co.

Marlin, T. E., Brisk, M. L., Barton, G. W. and Perkins, J. D. (2001). Experiences from an industry-university study on control benefits, Pulp and Paper Canada 102: 34-37.

Marlin, T. E., Perkins, J. D., Barton, G. W. and Brisk, M. L. (1987). Advanced Process Control Applications, Instrum. Society of America, Research Triangle Park N.C.

Marlin, T. E., Perkins, J. D., Brisk, M. L. and Barton, G. W. (1991). Benefits from process control: Results of a joint industry-university study, Journal of Process Control 1: 68-83.

Martin, G. D. (2004). Understand control benefits estimates, Hydrocarbon Processing 83(10): 43-46.

Martin, G. D., Turpin, L. E. and Cline, R. P. (1991). Estimating control function benefits, Hydrocarbon Processing 70: 68-73.

Martin, P. G. (2005). Bottom-line Automation, 2nd edn, ISA, pp. 17-52.

Mascio, R. D. (2002). An economic measure for comparing dynamic robustness, Journal of Process Control 12: 745-751.

Mascio, R. D. and Barton, G. W. (2001). The economic assessment of process control quality using a Taguchi-based method, Journal of Process Control 11: 81-88.

Mcmahon, T. K. (2004). Clifftent for process optimization, Control 17: 66-70.

Moore, D. S. and McCabe, G. P. (1989). Introduction to the Practice of Statistics, 2nd edn, New York: W. H. Freeman, pp. 153-172.

Mular, A. L. (1989). Automatic control of conventional and semi-autogenous grinding circuits., CIM Bulletin 82(922): 68-74.

Muller, B. and de Vaal, P. L. (2000). Development of a model predictive controller for a milling circuit, Journal of the South African Institute of Mining and Metallurgy 100(7): 449-453. 
Muller, B., Singh, A., de V van der Spuy, D. and Smith, V. C. (2003). Model predictive control in the minerals processing industry, in L. Lorenzen and D. J. Bradshaw (eds), Proceedings of the XXII International Mineral Processing Congress, Cape Town, South Africa, pp. 16921702.

Muske, K. R. (2003). Estimating the economic benefit from improved process control, Industrial and Engineering Chemistry Research 42: 4535-4555.

Muske, K. R. and Finegan, C. S. (2002). Analysis of a class of statistical techniques for estimating the ecomonic benefit from improved process control, Proceedings of the AIChE Symposium Series 326, Vol. 98, pp. 408-412.

Najim, K., Hodouin, D. and Desbiens, A. (1995). Adaptive control: State of the art and an application to a grinding process, Powder Technology 82: 59-68.

Napier-Munn, T. J. (1995). Detecting performance improvements in trials with time-varying mineral processes - three case studies, Minerals Engineering 8(8): 843 - 858.

Niemi, A. J., Tian, L. and Ylinen, R. (1995). A model predictive control for grinding systems, Proceedings of the IFAC Symposium on Automation in Mining, Mineral and Metal Processing, Sun City, South Africa, pp. 233-238.

Oosthuizen, D. J. (2001). Economic evaluation and design of an electric arc furnace controller based on economic objectives, Master's thesis, University of Pretoria.

Oosthuizen, D. J., Craig, I. K. and Pistorius, P. C. (2004). Economic evaluation and design of an electric arc furnace controller based on economic objectives, Control Engineering Practice 12: $253-265$.

Pomerleau, A., Hodouin, D., Desbiens, A. and Gagnon, E. (2000). A survey of grinding circuit control methods: From decentralized PID controllers to multivariable predictive controllers, Powder Technology 108: 103-115.

Punch, K. (2003). Survey Research: The Basics, London: SAGE.

Radhakrishnan, V. R. (1999). Model based supervisory control of a ball mill grinding circuit, Journal of Process Control 9(3): 195-211.

Ramasamy, M., Narayanan, S. S. and Rao, C. D. P. (2005). Control of ball mill grinding circuit using model predictive control scheme, Journal of Process Control 15(3): 273-283.

Rea, L. M. and Parker, R. A. (2005). Designing and Conducting Survey Research: A Comprehensive Guide, 3rd edn, Jossey-Bass.

Ross, S. A., Westerfield, R. W. and Jordan, B. D. (1992). Fundamentals of Corporate Finance, RICHARD D. IRWIN INC.

Samskog, P. O., Söderman, P., Storeng, U., Björkman, J., Guyot, O. and Broussaud, A. (1996). Model-based control of autogenous and pebble mills at LKAB Kiruna KA2 concentrator (Sweden), Proceedings of the International Autogenous and Semiautogenous Grinding Technology 1996, Vancouver, Canada, pp. 599-616.

Sapsford, R. (2007). Survey Research, London; Thousand Oaks, Calif. : SAGE.

Schutte, P. L. (2000). Private communication, Technical report, Iscor Steel, Pretoria. 
Seborg, D. E., Edgar, T. F. and Mellichamp, D. A. (2004). Process Dynamics and Control, 2nd edn, John Wiely and Sons.

Sienkiewica, J. R. (2002). Basic field instrumentation and control system maintenance in mineral processing circuits, in D. N. H. A. L. Mular and D. J. Barratt (eds), Mineral Processing Plant Design, Practice, and Control, Vol. 2, Society for Mining Metallurgy and Exploration, pp. 2104-2113.

Skogestad, S. (2003). Simple analytic rules for model reduction and PID controller tuning, Journal of Process Control 13(4): 291-309.

Skogestad, S. and Postlethwaite, I. (2005). Multivariable Feedback Control: Analysis and Design, John Wiley \& Sons, Ltd.

Smith, V. C., Hulbert, D. G. and Singh, A. (2001). AG/SAG control and optimization with PlantStar 2000, Proceedings of an International Conference on Autogenous and Semiautogenous Grinding Technology, Vancouver, Canada, pp. 282-293.

Sosa-Blanco, C., Hodouin, D., Bazin, C., Lara-Valenzuela, C. and Salazar, J. (1999). Integrated simulation of grinding and flotation application to a lead-silver ore, Minerals Engineering 12(8): 949-967.

Sosa-Blanco, C., Hodouin, D., Bazin, C., Lara-Valenzuela, C. and Salazar, J. (2000). Economic optimisation of a flotation plant through grinding circuit tuning, Minerals Engineering 13(1011): $999-1018$.

Sotelo, R. R., Gordon, L. M. and Jewart, C. (1997). Implementing advanced control on a copper ore grinding circuit, ISA TECH/EXPO Technology Update Conference Proceedings, Vol. 1, pp. 157-165.

Stange, W. and McInnes, C. (1995). The intelligent control of an ROM milling circuit, Proceedings of the IFAC Symposium on Automation in Mining, Mineral and Metal Processing, Sun City, South Africa, pp. 359-364.

Stanley, G. G. (1987). The Extractive Metallurgy of Gold in South Africa, Vol. 1, South African Institute of Mining and Metallurgy, Johannesburg.

Steane, H. A. (1976). Coarser grind may mean lower metal recovery but higher profits, Canadian Mining Journal 97: 44.

Stout, T. M. (1960). Evaluating control system payout from process data, Control Engineering $7(2): 67-70$.

Stout, T. M. and Cline, R. P. (1976). Control system justification, Instrumentation Technology 23: $51-58$.

Tabachnick, B. G. and Fidell, L. S. (2001). Using Multivariate Statistics, Allyn and Bacon.

Takatsu, H., Itoh, T. and Araki, M. (1998). Future needs for the control theory in industries report and topics of the control technology survey in Japanese industry, Journal of Process Control 8: 369-374.

Thomas, R. and Lynn, P. (2007). Survey Research in Practice, London: SAGE.

Thornhill, N. F. and Horch, A. (2007). Advances and new directions in plant-wide disturbance detection and diagnosis, Control Engineering Practice 15(10): 1196-1206. 
Tolfo, F. (1983). A methodology to estimate economic returns of advanced control projects, Proceedings of the American Control Conference, pp. 1141-1145.

Trahar, W. J. (1981). A rational interpretation of the role of particle size in flotation, International Journal of Mineral Processing 8: 289-327.

Trahar, W. J. and Warren, L. J. (1976). The flotability of very fine particles - a review, International Journal of Mineral Processing 3: 103-131.

van Dyk, W., Stange, W., Derbyshire, J. and Williams, H. (2000). Optimum control of the Leeudoorn semi-autogenous milling circuit, CIM Bulletin 93(1043): 106-110.

Vázquez, F. and Morilla, F. (2002). Tuning decentralized PID controllers for MIMO systems with decouplers, Proceedings of the 15th Trienial World Congress.

Wei, D. and Craig, I. K. (2009a). Development of performance functions for economic performance assessment of process control systems, Accepted by IEEE Africon 2009.

Wei, D. and Craig, I. K. (2009b). Economic performance assessment of two ROM ore milling circuit controllers, Minerals Engineering. 22: 826-839.

Wei, D. and Craig, I. K. (2009c). Grinding mill circuits - a survey of control and economic concerns, International Journal of Mineral Processing 90: 56-66.

Wei, D., Craig, I. K. and Bauer, M. (2007). Multivariate economic performance assessment of an MPC controlled electric arc furnace, ISA Transactions 46(3): 429-436.

Wills, B. A. and Napier-Munn, T. (2006). Mineral Processing Technology: an Introduction to the Practical Aspects of Ore Treatment and Mineral Recovery, Oxford: Butterworth-Heinemann.

Xu, F., Huang, B. and Akande, S. (2007). Performance assessment of model predictive control for variability and constraint tuning, Industrial and Engineering Chemistry Research 46: $1208-1219$.

Yahmedi, S., Pomerleau, A. and Hodouin, D. (1998). Robust controllers for grinding circuit, Proceedings of the IFAC Symposium on Automation in Mining, Mineral and Metal Processing, Germany, pp. 129-135.

Zhou, Y. (2002). Optimization-based benefits analysis for process control systems, Master's thesis, University of Alberta.

Zhou, Y. and Forbes, J. F. (2003). Determining controller benefits via probabilistic optimization, International Journal of Adaptive Control and Signal Processing 17: 553-568. 


\section{Appendix A}

\section{Pairing investigation using relative gain array}

In this part, the pairings of CVs and MVs for the milling circuit discussed in Chapter 7 are investigated using relative gain array (RGA). It turns out that from the RGA viewpoint, two options of pairings discussed in Section 7.2.3 are both not satisfactory. There is no considerable advantage of the parings of the PSD vs SFW and SLEV vs CFF, compared to the pairings of the PSD vs CFF and SLEV vs SFW.

\section{A.1 Basic relative gain array theory}

The RGA of a non-singular square complex matrix $G$ is defined as

$$
\operatorname{RGA}(G)=\wedge(G) \triangleq G \times\left(G^{-1}\right)^{T}
$$

where $\wedge$ means element-by-element multiplication. The RGA of a transfer function matrix is usually a function of frequency.

The RGA is a very useful tool in practical applications to reveal the intensity of the interactions between CVs and MVs of a process and to help determine the preferable pairings for decentralised control (Skogestad and Postlethwaite, 2005). There are two major pairing rules:

- Pairing rule 1: Prefer pairings such that the rearranged system, with the selected pairings along the diagonal, has an RGA matrix close to identity at frequencies around the closedloop bandwidth.

- Pairing rule 2: Avoid (if possible) pairings on negative steady-state RGA elements.

Large RGA elements at frequencies important for control imply that the process is essentially difficult to control owing to sensitivity to uncertainty and strong interactions. However, the magnitudes of RGA elements may be misleading and the phase of the RGA elements also need to be considered. RGA numbers are used for this purpose. A simple measure for choosing 
pairings according to rule $\mathbf{1}$ is to prefer pairings with a small RGA number. For diagonal pairings,

$$
\text { RGA number } \triangleq\|\wedge(G)-I\|_{\text {sum }}
$$

where $\|A\|_{\text {sum }}=\sum_{i, j}\left|a_{i j}\right|$. A more accurate declaration of pairing rule 1 would be to prefer pairings that bear diagonal dominance. There exists a close relation between diagonal dominance and a small RGA number.

\section{A.2 Relative gain array investigation for the milling cir- cuit}

For the linear model of the circuit discussed in Chapter 7, when the pairings are considered, there is no doubt that the MFS is used to control the LOAD. The decision in question is to use the CFF or SFW to control the PSD. The frequency model for the cyclone and sump is

$$
\left[\begin{array}{c}
\operatorname{PSD}(s) \\
\operatorname{SLEV}(s)
\end{array}\right]=\left[\begin{array}{cc}
-0.00035 \frac{(1-0.63 s)}{(1+0.54 s)} e^{(-0.011 s)} & \frac{0.00055}{(1+0.24 s)} e^{(-0.011 s)} \\
\frac{-0.29}{s} & \frac{0.42}{s}
\end{array}\right]\left[\begin{array}{c}
\operatorname{CFF}(s) \\
\operatorname{SFW}(s)
\end{array}\right]
$$

The first option of pairings is the PSD vs CFF and the SLEV vs SFW. Alternatively the second option is the PSD vs SFW and the SLEV vs CFF. The Matlab routines used to generate the RGA elements and number are given below:

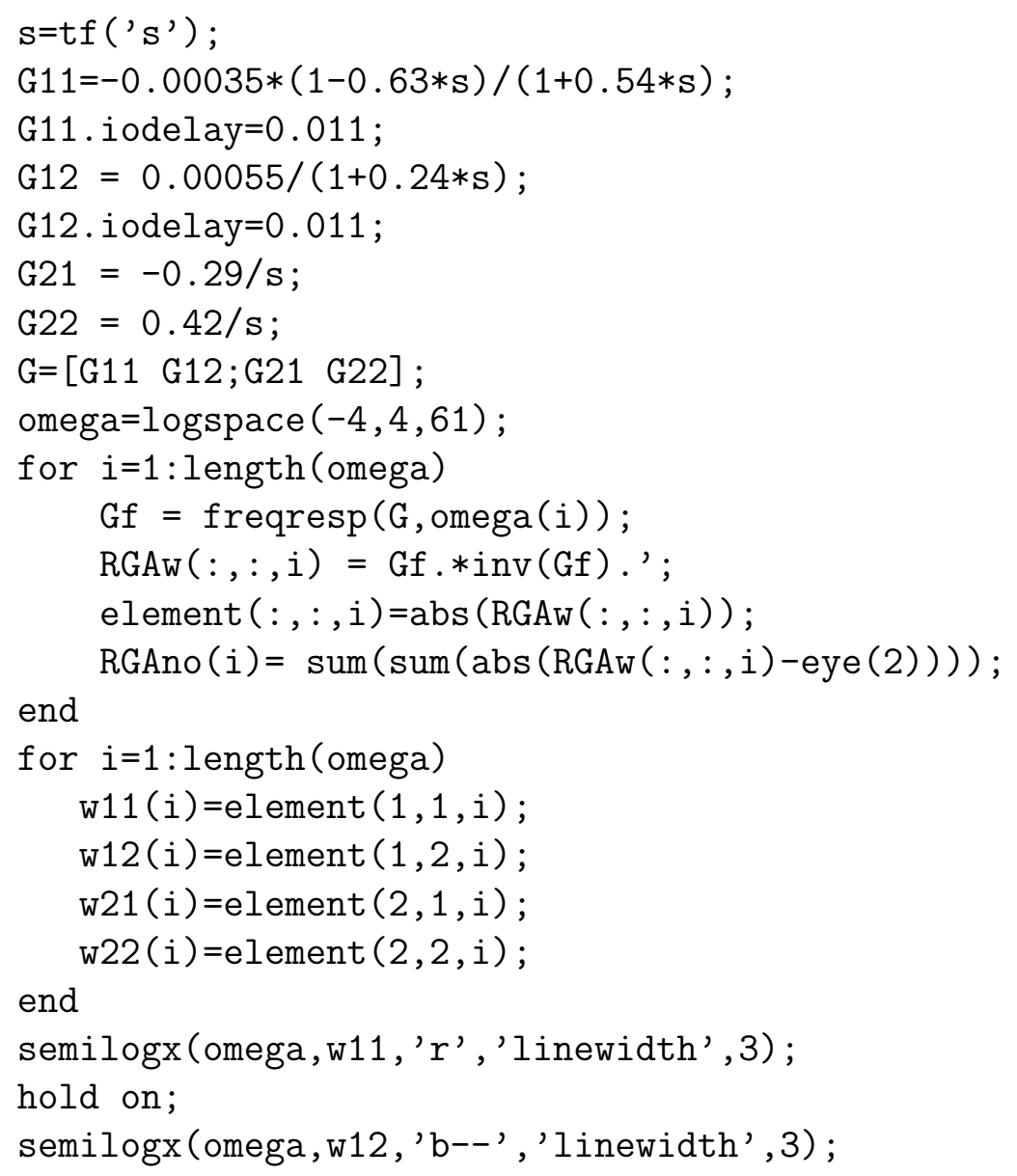


$\operatorname{xlim}([\min$ (omega) $\max$ (omega)]);

legend (' $\backslash$ lambda_ $\{11\}=\backslash$ lambda_$_{-}\{22\}$ ' , ' $\backslash$ lambda_ $\{12\}=\backslash$ lambda_$_{-}\{21\}$ ') ;

title('Magnitude of RGA elements');

grid on;

xlabel ('Frequency (rad/s)');

ylabel ('RGA elements');

saveas (gcf , 'Milling_RGAElement.eps' , 'epsc2') ;

figure;

semilogx (omega, RGAno, ' $r$ ' , 'linewidth', 3) ;

title('RGA number for different pairings');

hold on;

$\mathrm{G}=[$ G12 G11;G22 G21];

for $i=1$ : length (omega)

Gf $=$ freqresp $(G, \operatorname{omega}(i))$;

$\operatorname{RGAw}(:,:, i)=\operatorname{Gf} . * \operatorname{inv}(\mathrm{Gf}) . '$;

$\operatorname{element}(:,:, i)=\operatorname{abs}(\operatorname{RGAw}(:,:, i))$;

$\operatorname{RGAno}(i)=\operatorname{sum}(\operatorname{sum}(\operatorname{abs}(\operatorname{RGAw}(:,:, i)-$ eye $(2))))$;

end

semilogx (omega, RGAno, 'b-- ' , 'linewidth', 3) ;

legend ('PSD-CFF and SLEV-SFW', 'PSD-SFW and SLEV-CFF');

xlabel ('Frequency (rad/s)');

ylabel ('RGA number');

grid on;

saveas (gcf , 'Milling_RGANumber.eps' , 'epsc2');

The relevant RGA elements and number are given in Fig. A.1. The frequencies essential for

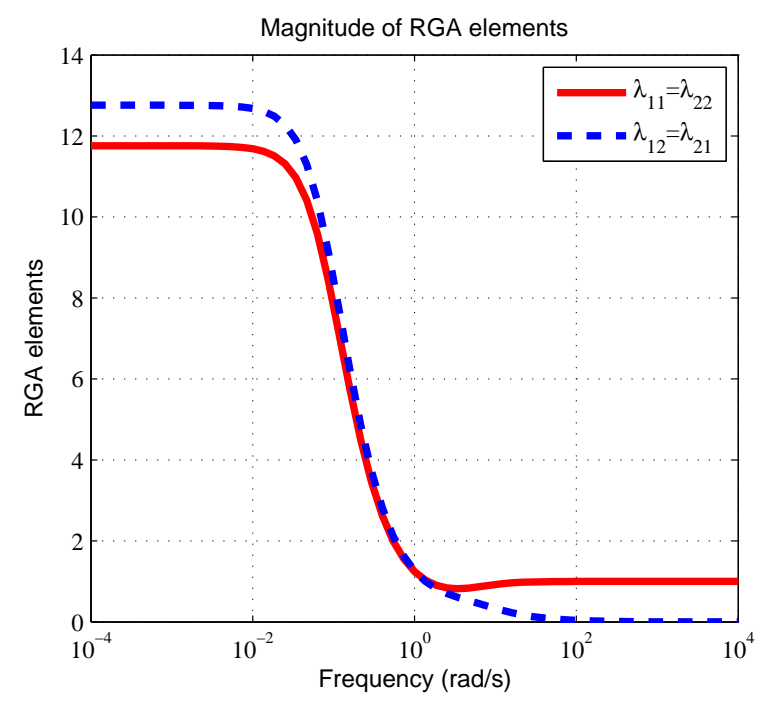

(a) RGA elements

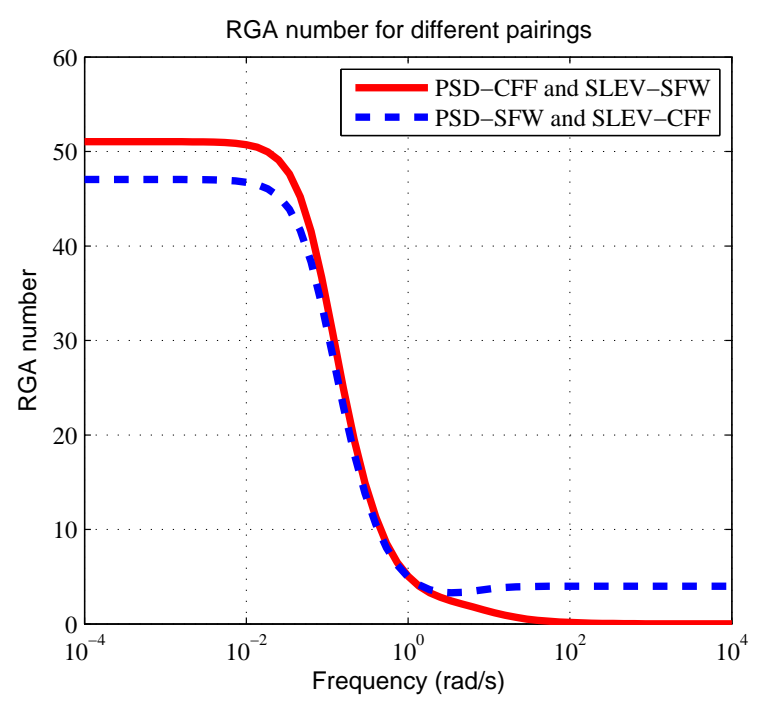

(b) RGA number

Figure A.1: RGA elements and number for the cyclone and sump model of the milling circuit

the control of the circuit being considered are at a lower interval. It can be seen from Fig. A.1 that at low frequency range the RGA elements for the two groups of pairings are both large and similar in magnitude. So is it for the RGA number. Therefore, as a conclusion, from RGA viewpoint, no one option is significantly better than the other one and both options of pairings are not satisfactory for decentralised control. 


\section{Appendix B}

\section{The derivation of minimum variance and relevant index}

In this part, the reasoning of the minimum variance control law for a single-input single-output (SISO) system is given firstly, followed by the filtering and correlation analysis algorithm used to obtain the control loop performance index.

\section{B.1 Feedback controller-invariance of minimum variance term}

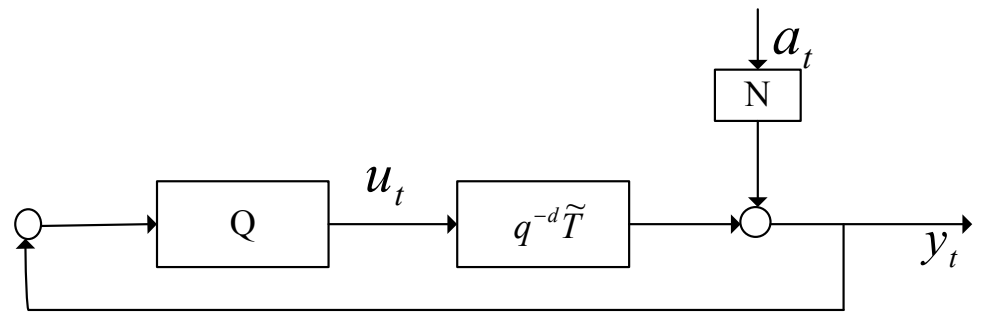

Figure B.1: Diagram of a SISO system with feedback control (Huang and Shah, 1999)

A SISO process, as given in Fig. B.1, is considered for regulatory control, where $d$ is time delay, $\tilde{T}$ is the delay-free plant transfer function, $N$ is the disturbance transfer function, $a_{t}$ is the zero-mean white noise time series and $Q$ is the controller transfer function. For the purpose of brevity and convenience, the backshift operator $q^{-1}$ will be omitted unless required. $T$, equivalent to $q^{-d} \tilde{T}$, is the process transfer function. The transfer function between the $y_{t}$ and $a_{t}$ is that

$$
y_{t}=\frac{N}{1+q^{-d} \tilde{T} Q} a_{t}
$$

when using the Diophantine identity:

$$
N=f_{0}+f_{1} q^{-1}+\cdots+f_{d-1} q^{-d+1}+R q^{-d}
$$


where $f_{i}$ (for $i=1, \cdots, d-1$ ) are coefficients, and $R$ is the remaining rational, proper transfer function. Eq. (B.1) can be denoted as

$$
\begin{aligned}
y_{t} & =\frac{F+q^{-d} R}{1+q^{-d} \tilde{T} Q} a_{t} \\
& =\left[F+\frac{R-F \tilde{T} Q}{1+q^{-d} \tilde{T} Q} q^{-d}\right] a_{t} \\
& =F a_{t}+L a_{t-d}
\end{aligned}
$$

where $L=\frac{R-F \tilde{T} Q}{1+q^{-d} \tilde{T} Q}$ is a proper transfer function. The two terms on the right-hand side of Eq. (B.3) are independent. Then

$$
\operatorname{Var}\left(y_{t}\right)=\operatorname{Var}\left(F a_{t}\right)+\operatorname{Var}\left(L a_{t-d}\right)
$$

So

$$
\operatorname{Var}\left(y_{t}\right) \geq \operatorname{Var}\left(F a_{t}\right)
$$

When the equality holds the minimum variance control law arises:

$$
Q=\frac{R}{\tilde{T} F}
$$

Because $F$ is independent of $Q$, the term $F a_{t}$, the process output with minimum variance control is not affected by the feedback controller. Then if a stable process output $y_{t}$ is modelled by an infinite moving-average model, its first $d$ terms can be used to estimate the minimum variance.

\section{B.2 The filtering and correlation algorithm for single- input single-output processes}

A stable closed-loop process can be expressed by an infinite-order moving-average process:

$$
y_{t}=\left(f_{0}+f_{1} q^{-1}+f_{2} q^{-2}+\cdots+f_{d-1} q^{-(d-1)}+f_{d} q^{-d}+\cdots\right) a_{t}
$$

Multiplying Eq. (B.7) by $a_{t}, a_{t-1}, \cdots, a_{t-d+1}$ respectively and then obtaining the expectation of both sides of the equation produces

$$
\begin{aligned}
r_{y a}(0) & =E\left[y_{t} a_{t}\right]=f_{0} \sigma_{a}^{2} \\
r_{y a}(1) & =E\left[y_{t} a_{t-1}\right]=f_{1} \sigma_{a}^{2} \\
r_{y a}(2) & =E\left[y_{t} a_{t-2}\right]=f_{2} \sigma_{a}^{2} \\
\vdots & \\
r_{y a}(d-1) & =E\left[y_{t} a_{t-d+1}\right]=f_{d-1} \sigma_{a}^{2}
\end{aligned}
$$

Therefore the controller invariant section of the output variance is

$$
\begin{aligned}
\sigma_{m v}^{2} & =\left(f_{0}^{2}+f_{1}^{2}+f_{2}^{2}+\cdots+f_{d-1}^{2}\right) \sigma_{a}^{2} \\
& =\left[r_{y a}^{2}(0)+r_{y a}^{2}(1)+r_{y a}^{2}(2)+\cdots+r_{y a}^{2}(d-1)\right] / \sigma_{a}^{2}
\end{aligned}
$$


Controller performance is defined here as

$$
\eta(d) \triangleq \sigma_{m v}^{2} / \sigma_{y}^{2}
$$

Substituting Eq. (B.9) into Eq. (B.10) produces

$$
\begin{aligned}
\eta(d) & =\left[r_{y a}^{2}(0)+r_{y a}^{2}(1)+r_{y a}^{2}(2)+\cdots+r_{y a}^{2}(d-1)\right] / \sigma_{y}^{2} \sigma_{a}^{2} \\
& =\rho_{y a}^{2}(0)+\rho_{y a}^{2}(1)+\rho_{y a}^{2}(2)++\cdots \rho_{y a}^{2}(d-1) \\
& =Z Z^{T}
\end{aligned}
$$

where $Z$ is the cross-correlation coefficient vector between $y_{t}$ and $a_{t}$ for lags 0 to $d-1$ and refers to

$$
Z \triangleq\left[\rho_{y a}^{2}(0), \rho_{y a}^{2}(1), \cdots, \rho_{y a}^{2}(d-1)\right]
$$

The relevant sampled version of the index is accordingly denoted as

$$
\hat{\eta}(d)=\hat{\rho}_{y a}^{2}(0)+\hat{\rho}_{y a}^{2}(1)+\hat{\rho}_{y a}^{2}(2)+\cdots \hat{\rho}_{y a}^{2}(d-1)=\hat{Z} \hat{Z}^{T}
$$

where

$$
\hat{\rho}_{y a}(k)=\frac{\frac{1}{M} \sum_{t=1}^{M} y_{t} a_{t-k}}{\sqrt{\frac{1}{M} \sum_{t=1}^{M} y_{t}^{2} \frac{1}{M} \sum_{t=1}^{M} a_{t}^{2}}}
$$

Although $a_{t}$ is unknown, the estimate can be used, which can be obtained by pre-whitening the process output variable $y_{t}$ using time series analysis. This algorithm is termed the FCOR algorithm for filtering and correlation analysis.

The variation in a regulatory closed-loop process may result from white noise excitation, $a_{t}$. The $y_{t}$ and $a_{t}$ are related by the closed-loop transfer function $G_{y a}=\frac{N}{1+T Q}$. The variation of $y_{t}$ is therefore caused by the excitation of $a_{t}$ through $G_{y a}$. The noise sequence estimate is important for functional performance assessment. The white noise sequence can be regarded as an output from a filter whose input is the process output $y_{t}$. Many methods have been developed to fit the filter model and obtain estimates of the white noise sequence from data of a process output variable. The process of obtaining such a 'whitening' filter is analogous to time-series modelling, where the examination of the sufficiency of the model involves investigating whether the residual are 'white'. The residuals are the estimated white noise sequence. Depending on data, an AR or ARMA can be used for estimating $a_{t}$. The identification of these innovation models has attracted much interest. Many efficient algorithms have been developed, such as arx, armax, etc in MATLAB. In this paper, arx is used to estimate the white noise. 


\section{List of Figures}

3.1 The framework of EPA (Bauer and Craig, 2008) . . . . . . . . . . 15

3.2 Frequently used PFs _. . . . . . . . . . . . . . . . . . . 17

3.3 PF of distillate composition (Friedmann, 2006) . . . . . . . . . . 18

3.4 Industrial use of advanced process control methods (Bauer and Craig, 2008) . 22

4.1 The structure of an EAF . . . . . . . . . . . . . . . 31

4.2 The EAF model variables (Oosthuizen et al., 2004) . . . . . . . . . . . . . 32

4.3 IPFs of the EAF . . . . . . . . . . . . . . . . . 33

4.4 Scatter plot of percentage carbon $c$ and off-gas temperature $t_{\text {og }} \ldots \ldots . \ldots$

4.5 Scatter plot of steel temperature $t_{s}$ and steel mass $m \ldots . . \ldots 38$

4.6 JPF of percentage carbon $c$ and off-gas temperature $t_{\text {og }} \ldots \ldots . \ldots$

4.7 JPF of steel temperature $t_{s}$ and steel mass $m \ldots \ldots . \ldots . \ldots 40$

4.8 Joint performance of off-gas temperature $t_{\text {og }}$ and percentage carbon $c$ under manual control . . . . . . . . . . . . . . . . . . . . 4 40

4.9 Joint performance of steel mass $m$ and steel temperature $t_{s}$ under manual control 41

5.1 A typical tumbling mill (Wills and Napier-Munn, 2006) . . . . . . . . . . 46

5.2 A two-stage closed circuit (Wills and Napier-Munn, 2006) . . . . . . . . . . 47

5.3 Continental distribution of the respondents . . . . . . . . . . . . . . 58

5.4 The positions of the respondents . . . . . . . . . . . . . . . . 59

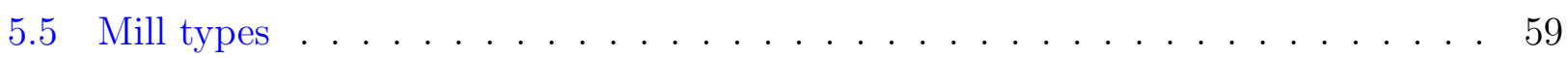


5.7 Mill discharge f. . . . . . . . . . . . . . . . . . . . . . . . 59

5.8 Circuit configuration . . . . . . . . . . . . . . . . . . . 59

5.9 Classification instruments of a closed circuit . . . . . . . . . . . . . . 60

5.10 Minerals in the mill feed . . . . . . . . . . . . . . . . . 60

5.11 Grinding media . . . . . . . . . . . . . . . . . . . . 60

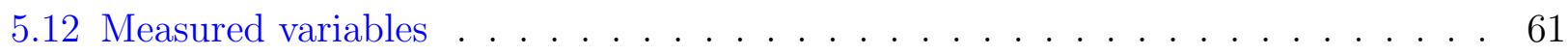

5.13 Method for measuring product particle size . . . . . . . . . . . . . 61

5.14 Method for measuring mass of mill load . . . . . . . . . . . . . . . 61

5.15 Method for measuring volume of mill load . . . . . . . . . . . . . . 62

5.16 Method for measuring density of sump discharge . . . . . . . . . . . . . . 62

5.17 Method for measuring flow rate of water and slurry . . . . . . . . . . . 62

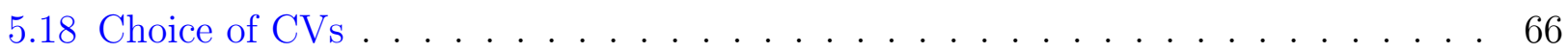

5.19 Choice of manipulated variables . . . . . . . . . . . . . . 66

5.20 Considerations when choosing setpoint for product particle size $\ldots \ldots$. . . . 67

5.21 Action taken to grind finer . . . . . . . . . . . . . . . . . 67

5.22 Action taken to grind coarser . . . . . . . . . . . . . . 67

5.23 Qualitative economic impact of grinding finer than expected . . . . . . . . 68

5.24 Corrective action for too high a sump level . . . . . . . . . . . . . 68

5.25 Economic impact of too high sump level . . . . . . . . . . . . . . 68

5.26 Economic impact of too low sump level . . . . . . . . . . . . . . . . . . 69

5.27 Economic impact of too high a mill load . . . . . . . . . . . . . . 69

5.28 Economic impact of too low a mill load f . . . . . . . . . . . . . . . . 69

5.29 Corrective action for a too low sump discharge slurry density . . . . . . . . 70

5.30 The options of $\mathrm{PFs} \ldots \ldots \ldots \ldots$ 
5.31 Economic impact of the CVs deviating from the setpoints. The (a), (b), (c), (d), (e), and (f) here refer to the subplots in Fig. 5.30 respectively

5.32 Dependence of $\mathrm{CVs} \ldots \ldots \ldots \ldots \ldots \ldots$

5.33 Importance of economic analysis . . . . . . . . . . . . . . . . 73

5.34 Accuracy of economic analysis . . . . . . . . . . . . . . . . 74

5.35 Satisfaction with economic analysis . . . . . . . . . . . . . 74

5.36 Topics requiring additional development . . . . . . . . . . . . . . 74

6.1 Economic impact of particle size (Craig et al., 1992b) . . . . . . . . . 78

6.2 The structure of the milling circuit (adapted from Coetzee et al. (2008)) . . . . 79

6.3 Recovery vs size fraction relation (from Lynch and Bush (1977)) . . . . . . . 80

6.4 PF (recovery) of the ore PSD of the milling circuit product (adapted from Fig. 7 in Edwards and Vien (1999)) . . . . . . . . . . . . . 81

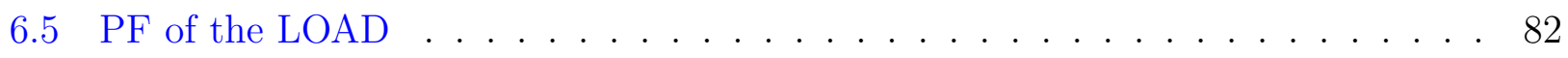

$6.6 \mathrm{PF}$ of the SLEV . . . . . . . . . . . . . . . . . 83

7.1 The modules of the circuit model (Coetzee et al., 2008) . . . . . . . . . . 86

7.2 CVs responses to the multiple step tests of the CFF . . . . . . . . . . 94

7.3 CVs responses to the multiple step tests of the SFW. No linear relation between the LOAD and SFW is revealed from the system identification based on the relevant step tests and accordingly no comparison is done for them. . . . . . . .

7.4 CVs responses to the multiple step tests of the MFS. No linear relation between the SLEV and MFS is revealed from the system identification based on the relevant step tests and accordingly no comparison is done for them. . . . . . . . 95

7.5 Relative gain array elements of the circuit model . . . . . . . . . . . . 98

7.6 Singular values as function of frequency for the circuit model $G \ldots \ldots$

7.7 CVs and MVs with SIMC PID controllers with stationary centred disturbance . 102

7.8 CVs and MVs of one-day operation for the SIMC PID controllers under persistent and random disturbances . . . . . . . . . . . . . . . 103

7.9 CVs and MVs of one-day operation for the revised SIMC PID controllers with error squared control for the SLEV under persistent and random disturbances 
7.10 The scheme of the decoupling system (Seborg et al., 2004) . . . . . . . . . 107

7.11 The Simulink block for the decentralised controller . . . . . . . . . . . . . 109

7.12 CVs and MVs with stationary centred disturbance for the decentralised controller 110

7.13 CVs and MVs with persistent and stationary centred disturbances for the decentralised controller . . . . . . . . . . . . . . . . 111

7.14 Control technologies used in milling circuits . . . . . . . . . . . . . 113

7.15 CVs and MVs of one-day operation for the NMPC controller under stationary centred disturbances . . . . . . . . . . . . . . . . . 116

7.16 CVs and MVs of one-day operation for the NMPC controller under persistent plus stationary disturbances . . . . . . . . . . . . . . . . 117

7.17 CVs of three controllers under two disturbance scenarios . . . . . . . . . . 118

7.18 Cost types of a process control system . . . . . . . . . . . . . . . 121

7.19 Scatter plot for the THR and PSD . . . . . . . . . . . . . . . 127

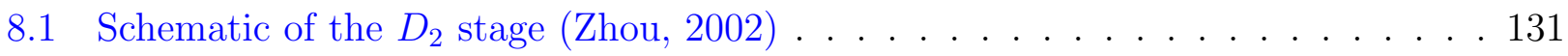

8.2 Performance function of brightness . . . . . . . . . . . . . . 132

A.1 RGA elements and number for the cyclone and sump model of the milling circuit 156

B.1 Diagram of a SISO system with feedback control (Huang and Shah, 1999) . . . 157 


\section{List of Tables}

4.1 Summary of assessment. . . . . . . . . . . . . . . . . . . . 43

5.1 Methods for measuring other variables . . . . . . . . . . . . . 63

5.2 Methods for manipulating variables (\% is used to express the percentage of respondents using a particular actuator type) . . . . . . . . . . 63

5.3 Troublesome instruments and reasons . . . . . . . . . . . . . . . . . 64

5.4 Instrument wish list . . . . . . . . . . . . . . . . . . . 64

6.1 Notation of symbols used in expressing performance . . . . . . . . . . . 83

7.1 The milling circuit model's nomenclature . . . . . . . . . . . . . . . 87

7.2 Operating point and constraints (OP means operating point) of the process states 91

7.3 Operating point and constraints (OP means operating point) of the MVs and CVs 91

7.4 Parameter values for the milling circuit ( $\Delta$ means the percentage value of the difference between the biggest uncertainty and the nominal value divided by the

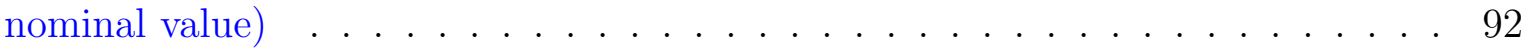

7.5 Summary of simulation results with stationary centred disturbances under SIMC PID controllers . . . . . . . . . . . . . . . . . . . 102

7.6 Summary of simulation results with stationary centred and persistent disturbances under SIMC PID controllers . . . . . . . . . . . . . . . . . 103

7.7 Summary of simulation results with stationary centred and persistent disturbances under SIMC PID (and error squared) controllers . . . . . . . . . . . . . . 104

7.8 Summary of simulation results with stationary centred disturbances under the decentralised controller . . . . . . . . . . . . . . . 110

7.9 Summary of simulation results with persistent and stationary centred disturbances under the decentralised controller . . . . . . . . . . . . . . . . . . 111 
7.10 Statistical data of CVs for the NMPC controller under stationary and persistent disturbances . . . . . . . . . . . . . . . . . . . 117

7.11 Summary of simulation results of the SIMC PID controllers, decentralised controller and NMPC controller under two disturbance scenarios: Scenario 1 refers to stationary centred disturbances and Scenario 2 refers to stationary and persistent disturbances. . . . . . . . . . . . . . . . . . . . . . 118

7.12 Summary of simulation results of statistical experiments . . . . . . . . . . 123

9.1 Operational cost distribution for an electric arc furnace (Oosthuizen et al., 2004) 136

9.2 Economic consequences associated with $y_{i} \ldots \ldots \ldots$. . . . . . . . . 136 